\title{
A Unified De Novo Approach for Predicting the Structures of Ordered and Disordered Proteins
}

\section{Supplementary Information}

\author{
J. J. Ferrie* and E. J. Petersson* \\ Department of Chemistry, University of Pennsylvania, 231 South 34th Street, Philadelphia, \\ Pennsylvania 19104-6323, USA \\ *Email to JJF: jferrie@sas.upenn.edu or EJP: ejpetersson@sas.upenn.edu
}

\section{Table of Contents}

General Information...

Computational Resources

Generalized Simulation Format. S2

Construction of Fragment Libraries. S3

Calculation of Data from Ensembles..... S4

FRET Data S4

Distance Data S4

PRE Data S4

Chemical Shift Data S5

Residual Dipolar Couplings.... S5

J-Couplings . 55

Percent Helicity . 56

Comparison of $\alpha \mathrm{S}$ Ensembles from Generalized Simulation to Experimental Data . 57

Summary of Generalized Simulations of $\alpha \mathrm{S}$ S7

Comparison of $\alpha$ S Per Residue Percent Helicity ..... S11

Comparison with $\alpha \mathrm{S}$ Radius of Gyration Data S13

Comparison with aS FRET Data. S15

Comparison with $\alpha \mathrm{S}$ Distance Data S17

Comparison with $\alpha$ S PRE Data. S19

Comparison with $\alpha \mathrm{S}$ Chemical Shift Data..... S48

Comparison with aS Residual Dipolar Coupling Data. S77

Comparison with $\alpha \mathrm{S}$ J-Coupling Data S85

Construction of the New Rg Score Term. S114

Implementation of the New Rg Score Term in PyRosetta 
Determination of the Optimal Weight for the New Rg Score Term ............................................. S116

Comparison of AbInitio and AbInitioVO Simulations to Experimental Data ...................................... S117

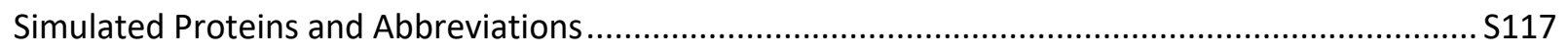

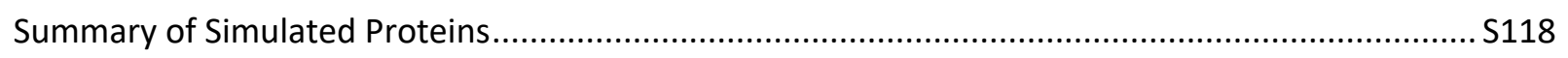

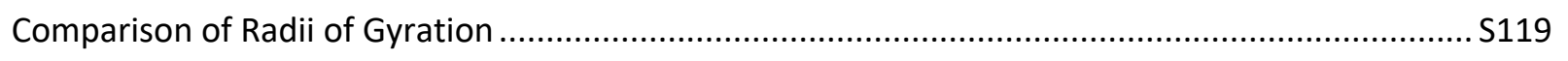

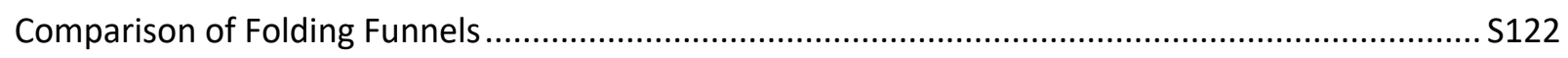

Comparisons of Folded Domain Structures of Ordered and Partially-Ordered Proteins ................. S134

Structural Comparisons of Disordered Domains of Partially-Ordered and Disordered Proteins ....... S140

Comparison of FastFloppyTail and FastFloppyTail-Relax Simulations to Experimental Data................ S145

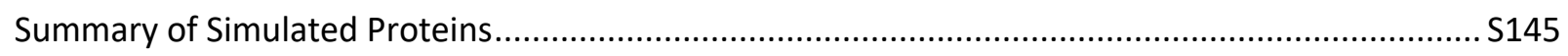

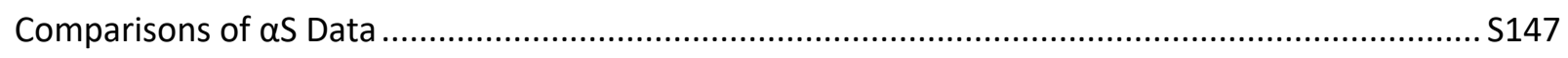

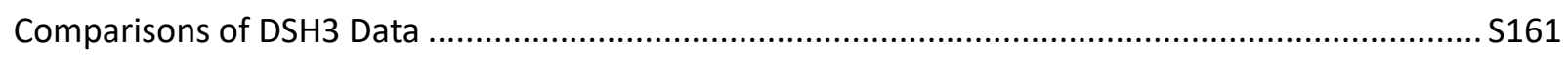

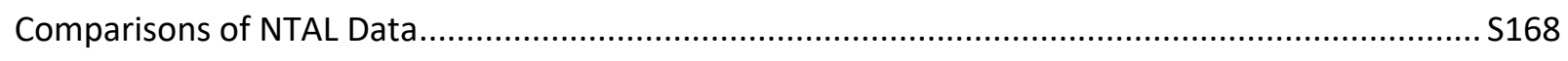

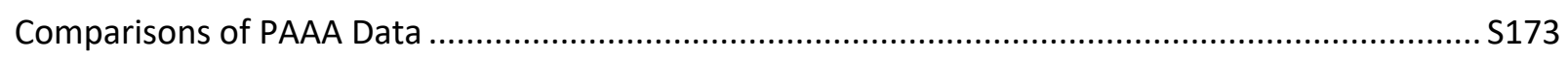

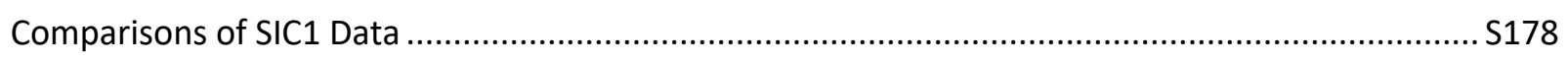

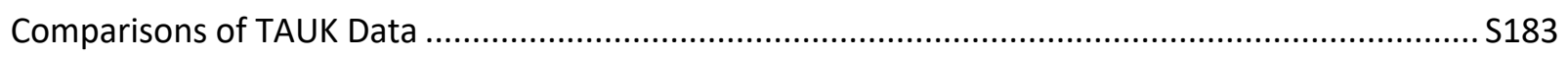

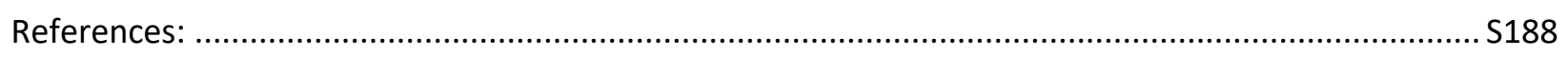

\section{General Information}

Computational Resources: All simulations were performed on the University of Pennsylvania School of Arts and Sciences General Purpose Cluster (GPC). Each of the four GPC compute nodes contains 24 cores at $2.6 \mathrm{Ghz}, 192 \mathrm{~GB}$ RAM, 1 GbE Networking, 56 Gb Infiniband and 300 GB $\mathrm{OS} / \mathrm{Scratch}$ storage. All times reported herein were from 140-residue $\alpha \mathrm{S}$. On average, Full-atom Generalized Simulations required $\sim 3$ hours per structure while simulations using exclusively Centroid coarse-graining required $\sim 30$ minutes per structure. Simulations using both coarsegrained and all-atom molecular representations (SimAnn) took $\sim 1.5$ hours per structure and the previously reported FloppyTail simulation method took $\sim 30$ minutes per structure whereas FastFloppyTail simulation method took $\sim 3$ minutes per structure. The AbInitio and AbInitioVO algorithms both required $\sim 45$ minutes per structure and Relaxes took $\sim 3$ minutes a structure.

Generalized Simulation Format: We chose to sample the effects of full-atom and centroid AtomTypes on the simulation, in an effort to assess the possibility of using the coarse-grained method to speed up the simulation during initial sampling steps as is frequently done in Rosetta. Additionally, we assessed the utility of different score terms and focused on six different score weights (VDW: exclusively repulsive van der Waals term "fa_rep", CenStd: "cen_std" score term weights, CenStdExt: "cen_std" score term weights adding "rama", "cenpack", "hbond_lr_bb" and "hbond_sr_bb" all at weights of 1.0, Beta: "beta_nov15" score term weights, SimAnn: simulated 
annealing where additional score terms were included as sampling progressed utilizing "score0", "score1", "score2", "score3" and "beta_nov15", CenNath: "vdw", "rama", "pair", "env", "hbond_lr_bb" and "hbond_sr_bb" all at weights of 1.0, and CenOpt: identical to the SimAnn centroid score functions with "rg" at a weight of 0 and "hbond_lr_bb" and "hbond_sr_bb" at weights of 1.0). ${ }^{1}$ Finally, we utilized three different types of Movers within each of the different AtomType/ScoreFunction combinations (PP: $\varphi / \psi$ torsion angle changes using Small/Shear Movers, FI: fragment insertion, and SC: side-chain rotamer optimization using the PackRotamersMover). The simulation nomenclature is in the form AtomType_Scoring_Sampling. For example, FA_Beta_PPFI utilizes the full-atom AtomType, the "beta_nov15" scoring term weights for the ScoreFunction and application of both phi/psi sampling and fragment insertion. To assess the impact of each of these parameters on the resultant ensemble, a base script was devised which could be easily altered to analyze variable effects while keeping the number of Movers applied to the protein backbone over the course of the simulation constant. In brief, the method consisted primarily of RandomMovers, which select and apply a single move from a detailed set of movers comprised of SmallMovers and ShearMovers (apply $\varphi / \psi$ torsion angle changes), as well as MinMovers (perform gradient-descent minimization to locate local minima). Fragment insertion in the form of the ClassicFragmentMover was added directly to RandomMovers for both 9-mer and 3-mer fragments. Side-chain sampling was not added directly to the RandomMover, but was applied after a move from the RandomMover. The number of moves applied by the RandomMover prior to application of the Metropolis-Hastings acceptance criteria were decreased over the course of the simulation. The temperature (specified kT) value was also decreased. Lastly, the structure was set to the lowest energy structure found after each sampling stage and at the end of the simulation. For each simulation the resultant ensembles consisted of $\sim 1000$ structures.

Construction of Fragment Libraries: The Robetta server was used to generate the initial fragment library for testing all variants of the "Generalized Simulation" along with "FloppyTail" and "FloppyTail_ref2015". ${ }^{2}$ Production of the custom fragment libraries used were prepared using the FragmentPicker application in Rosetta ${ }^{3}$. All fragment libraries contained 200 fragments. The "FloppyTail_Quota" library was prepared using secondary structure probability predictions from the primary sequence of $\alpha$-synuclein $(\alpha \mathrm{S})$ using the Jufo, PsiPred and RaptorX servers along with the quota protocol of the FragmentPicker application..$^{4-6}$ The probability of selecting fragments based on Jufo, PsiPred and RaptorX predictions were set to $0.33,0.33$ and 0.34 respectively with priorities for SecondarySimilarity of 250, 300 and 350 with weights of 1.0 for all and priorities ofor RamaScore of 150 with a weight of 2.0 for all predictions in the input weight file. The input weight file also contained ProfileScoreL1 with priority and weight of 200 and 2.0 respectively based on a sequence profile from PSI-BLAST. The "FloppyTail_Loop" library was prepared by exchanging the secondary structure predictions with a single manually crafted prediction input that contains 1.0 loop probabilities at every residue of the sequence. All "FloppyTail" simulations not previously addressed, including all FastFloppyTail simulations in the Generalized Simulation section, utilized a quota-style fragment library using the same inputs as detailed above, where the disordered probability prediction was used to re-weight loop contributions as described in the main text based on a disordered probability prediction from RaptorX. 
AbInitio and AbInitioVO simulations were generated using the FragmentPicker following the best protocol, using only PsiPred secondary structure predictions as inputs. AbInitioVO PsiPred predictions were loop reweighted using disordered probability predictions from RaptorX, termed best-reweighting, as described in the main text. FastFloppyTail simulations of $\alpha \mathrm{S}$ and other disordered proteins were performed using libraries generated using the quota protocol of the FragmentPicker application and the best-reweighting approach, identical to that employed for AbInitioVO simulations. This contained SecondarySimilary (priority: 350, weight 1.0), RamaScore (priority: 150, weight: 2.0) and ProfileScoreL1 (priority: 200, weight: 2.0) as inputs. Libraries were also generated using self-reweighting, where secondary structure predictions from PsiPred and RaptorX were reweighted based on disordered probability predictions from PsiPred and RaptorX respectively. Additionally, all residues were set to probabilities of being $100 \%$ loop unless, following reweighting, the residue helix or loop probability was $>0.75$ and at least 3 or 5 consecutive residues were identified as sheets or helices respectively. The input file values were adjusted accordingly (PsiPred:: SecondarySimilary: priority: 300 weight: 1.0, RamaScore: priority: 150 weight: 2.0 , RaptorX:: SecondarySimilary: priority: 350 weight: 1.0, RamaScore: priority: 150 weight: 2.0 ).

\section{Calculation of Data from Ensembles}

FRET Data: FRET efficiencies (EFRETS) for each residue pair were computed for each individual structure and averaged across all structures in a given ensemble to determine the average EFRET of the ensemble. This was performed through application of the Förster Equation:

$$
E_{F R E T}=\frac{1}{1+\left(r / R_{0}\right)^{6}}
$$

which is dependent on the interfluorophore distance, $r$, and the Förster distance $\left(R_{0}\right)$. $R_{0}$ values were taken from the references listed with figures referring to FRET data. For expedience, we approximated $r$ as the distance between the $\mathrm{C} \alpha$ atoms of the labeled residues.

Distance Data: Distances for each residue pair were determined from the distances between the $\mathrm{C} \alpha$ atoms of each residue for each structure in each ensemble and were averaged over all members of the ensemble.

PRE Data: Paramagnetic relaxation enhancement (PRE) values $\left(\Gamma_{2}\right)$ were computed and converted to the experimentally observed value $\left(I_{o x} / I_{\text {red }}\right)$ from each structure in the ensemble using the formulas:

$$
\begin{gathered}
\Gamma_{2}=\left[\frac{K}{r^{6}}\left(4 \tau_{c}+\frac{3 \tau_{c}}{1+\omega^{2} \tau_{c}^{2}}\right)\right] \\
\frac{I_{o x}}{I_{\text {red }}}=\frac{R 2_{\text {red }} \exp \left(-\Gamma_{2} t\right)}{R 2_{\text {red }}+\Gamma_{2}}
\end{gathered}
$$

previously detailed by Sung et al. ${ }^{8}$ Here, $K$ is a constant that describes the spin properties for the nitroxide radical $\left(1.23 \times 10^{-32} \mathrm{~cm}^{6} \mathrm{~s}^{-2}\right), \tau_{c}$ is the correlation time for the electron-nuclear interaction vector (4 ns) and $\omega / 2 \pi$ is the Larmour frequency of an amide proton (computed in all cases for a $700 \mathrm{MHz}$ field), $R 2_{\text {red }}$ is the transverse relaxation rate in the diamagnetic state (set to $4 \mathrm{~s}^{-1}$ ) and $t$ is the total INEPT evolution time of the HSQC experiment, which was $10 \mathrm{~ms}$ for comparisons to 
data from Dedmon et al. ${ }^{9}$ (as previously done by Piana et al. ${ }^{10}$ ) and $4 \mathrm{~ms}$ for comparisons to data from Sung et al. ${ }^{8}$.

Chemical Shift Data: The amide proton $(\mathrm{HN})$, amide nitrogen $(\mathrm{N})$, carbonyl carbon $(\mathrm{C})$, $\alpha$-carbon $(\mathrm{C} \alpha), \alpha$-carbon proton (HA), and $\beta$-carbon $(\mathrm{C} \beta)$ chemical shifts were all computed using the SPARTA+ package developed by Shen and Bax. ${ }^{11}$ The chemical shift values were computed for each structure and averages were computed uniformly across a given ensemble. Secondary chemical shifts were calculated by subtracting random coil chemical shifts for each residue as assigned by SPARTA $+{ }^{11}$.

Residual Dipolar Couplings: All residual dipolar couplings (RDCs) were computed using PALES ${ }^{12}$. For each structure in a given ensemble, the RDC for a given residue was computed using the "-bestFit" flag along suppling input data for comparison. Calculations were performed on 15 residue segments where the residue of interest occupied the central position, as previously done by Piana et al. ${ }^{10}$. For the first and last seven residues in a given protein, the $\mathrm{N}$ - and C-terminal 15 residue segments were used. The averages were computed for the 1000 lowest energy structures in each ensemble.

J-Couplings: The NMR $J$-couplings were calculated using fitted Karplus equations previously utilized by Shen and Bax from backbone $\varphi / \psi$ dihedral angles ${ }^{13}$. For each structure, $\varphi$ and $\psi$ were computed in DSSP ${ }^{14}$. As with other values, the $J$-coupling was computed for each residue in each structure and averaged across all members of the ensemble. The Karplus equations used to compute each $J$-coupling are detailed below:

$$
\begin{gathered}
{ }^{3} J_{H N-H A}=7.97 \cos ^{2}\left(\varphi-60^{\circ}\right)-1.26 \cos \left(\varphi-60^{\circ}\right)+0.63 \\
{ }^{1} J_{C \alpha-H \alpha}=A_{R C}+1.4 \sin \left(\psi+138^{\circ}\right)-4.1 \cos \left(2\left[\psi+138^{\circ}\right]\right)+1.7 \cos \left(2\left[\varphi+60^{\circ}\right]\right) \\
{ }^{1} J_{C \alpha-N}=9.5098-0.9799 \cos (\psi)+1.7040 \cos ^{2}(\psi) \\
{ }^{2} J_{C \alpha-N}=C-1.5176 \cos (\psi)-0.2047 \cos ^{2}(\psi) \\
{ }^{3} J_{C^{\prime}-C^{\prime}}=0.46-0.95 \cos (\psi)+1.78 \cos ^{2}(\psi)
\end{gathered}
$$

For both the ${ }^{1} J_{\mathrm{C} \alpha-\mathrm{H} \alpha}$ and the ${ }^{2} J_{\mathrm{C} \alpha-\mathrm{N}}$ couplings, the value of the constants $A_{R C}$ and $C$ are amino acid dependent. For Val, Thr, Ile, and Ser $C=7.65$ while for all other amino acids, $C=8.15^{13}$. The constant $A_{R C}$ values are listed in Table $\mathrm{S} 1$ below and originate from the random coil ${ }^{1} J_{\mathrm{C} \alpha-\mathrm{H} \alpha}$ values 16 .

Table S1.

\begin{tabular}{llllllll}
\hline Residue & $\boldsymbol{A}_{\boldsymbol{R} \boldsymbol{C}}$ & Residue & $\boldsymbol{A}_{\boldsymbol{R} \boldsymbol{C}}$ & Residue & $\boldsymbol{A}_{\boldsymbol{R} \boldsymbol{C}}$ & Residue & $\boldsymbol{A}_{\boldsymbol{R} \boldsymbol{C}}$ \\
\hline Ala & 143.7 & Glu & 141.9 & Met & 142.2 & Trp & 143.0 \\
Arg & 141.5 & His & 143.8 & Phe & 142.9 & Tyr & 143.0 \\
Asn & 141.5 & Ile & 141.3 & Pro & 148.4 & Val & 141.3 \\
Asp & 142.5 & Leu & 141.1 & Ser & 142.1 & Other & 140.3 \\
Gln & 141.1 & Lys & 141.5 & Thr & 141.4 & & \\
\hline
\end{tabular}


Percent Helicity: For each structure, secondary structures were assigned using DSSP ${ }^{14}$. Residues assigned as a $3_{10}$ helix, $\alpha$-helix or $\pi$-helix, corresponding to G, H and I assignments in DSSP respectively, were counted as helical. 


\section{Comparison of $\alpha \mathrm{S}$ Ensembles from Generalized Simulation to Experimental Data}

\section{Summary of Generalized Simulations of $\alpha \mathrm{S}$}

Table S2: Comparison of Simulated $\alpha$ S Ensembles to Global Experimental Data.

\begin{tabular}{|c|c|c|c|c|c|c|c|c|c|}
\hline \multirow[b]{2}{*}{ Name } & \multirow[b]{2}{*}{ AtomType } & \multirow[b]{2}{*}{ ScoreFunction } & \multicolumn{3}{|c|}{ Sampling } & \multirow[b]{2}{*}{$\operatorname{Rg}(\AA ̊)$} & \multirow{2}{*}{$\begin{array}{c}\text { EFRET } \\
\text { RMSD }\end{array}$} & \multirow{2}{*}{$\begin{array}{c}I o / I_{R} \\
\text { RMSD }\end{array}$} & \multirow{2}{*}{$\begin{array}{c}\text { Dist. } \\
\text { RMSD }\end{array}$} \\
\hline & & & PP & FI & SC & & & & \\
\hline \multicolumn{10}{|c|}{ Generalized Simulation } \\
\hline VDW_PP & FA & fa_rep & $\mathrm{X}$ & & & $43.7+8.0$ & 0.18 & 0.23 & 17.5 \\
\hline VDW_PPFI & FA & fa_rep & $\mathrm{X}$ & $\mathrm{X}$ & & $39.7+6.8$ & 0.15 & 0.21 & 13.17 \\
\hline VDW_PPFISC & FA & fa_rep & $\mathrm{X}$ & $\mathrm{X}$ & $\mathrm{X}$ & $40.0+7.0$ & 0.15 & 0.21 & 14.0 \\
\hline CenStd_PP & CEN & cen_std & $\mathrm{X}$ & & & $19.6+2.5$ & 0.28 & 0.20 & 11.9 \\
\hline CenStd_PPFI & CEN & cen_std & $\mathrm{X}$ & $\mathrm{X}$ & & $19.2+2.5$ & 0.28 & 0.22 & 12.5 \\
\hline CenNath & CEN & CenNath & $\mathrm{X}$ & & & $33.4+7.9$ & 0.11 & 0.18 & 8.09 \\
\hline CenStd_Ext_PP & CEN & CenStdExt & $\mathrm{X}$ & & & $32.7+8.7$ & 0.11 & 0.17 & 7.02 \\
\hline CenStd_Ext_PPFI & CEN & CenStdExt & $\mathrm{X}$ & $\mathrm{X}$ & & $25.6+5.6$ & 0.17 & 0.16 & 6.69 \\
\hline Beta_PP & FA & ref2015 & $\mathrm{X}$ & & & $30.6+5.5$ & 0.13 & 0.17 & 8.23 \\
\hline Beta_PPFI & FA & ref2015 & $\mathrm{X}$ & $\mathrm{X}$ & & $36.4+6.9$ & 0.12 & 0.19 & 11.7 \\
\hline Beta_PPFISC & FA & ref2015 & $\mathrm{X}$ & $\mathrm{X}$ & $\mathrm{X}$ & $40.5+6.7$ & 0.18 & 0.20 & 15.2 \\
\hline Beta_PPSC & FA & ref2015 & $\mathrm{X}$ & & $\mathrm{X}$ & $30.3+5.3$ & 0.13 & 0.17 & 7.74 \\
\hline SimAnn_PP & CEN/FA & score0-3/ref2015 & $\mathrm{X}$ & & & $33.9+7.0$ & 0.10 & 0.18 & 8.54 \\
\hline SimAnn_PPFI & CEN/FA & score $0-3 /$ ref2015 & $\mathrm{X}$ & $\mathrm{X}$ & & $33.4+6.8$ & 0.11 & 0.18 & 9.02 \\
\hline SimAnn_PPFISC & CEN/FA & score0-3/ref2015 & $\mathrm{X}$ & $\mathrm{X}$ & $\mathrm{X}$ & $25.5+4.0$ & 0.18 & 0.17 & 8.14 \\
\hline SimAnn_PPSC & CEN/FA & score0-3/ref2015 & $\mathrm{X}$ & & $\mathrm{X}$ & $31.7+6.3$ & 0.12 & 0.17 & 7.38 \\
\hline \multicolumn{10}{|c|}{ FloppyTail } \\
\hline FloppyTail_score12 & $\mathrm{CEN} / \mathrm{FA}$ & CenStdExt/score12 & $\mathrm{X}$ & $\mathrm{X}$ & $\mathrm{X}$ & $32.8+6.0$ & 0.12 & 0.18 & 8.36 \\
\hline FloppyTail_ref2015 & CEN/FA & CenStdExt/ref2015 & $\mathrm{X}$ & $\mathrm{X}$ & $\mathrm{X}$ & $33.9+6.4$ & 0.12 & 0.18 & 9.39 \\
\hline FloppyTail_Quota & CEN/FA & CenStdExt/ref2015 & $\mathrm{X}$ & $\mathrm{X}$ & $\mathrm{X}$ & $34.2+6.1$ & 0.12 & 0.18 & 9.02 \\
\hline FloppyTail_Loops & CEN/FA & CenStdExt/ref2015 & $\mathrm{X}$ & $\mathrm{X}$ & $\mathrm{X}$ & $35.3+6.7$ & 0.12 & 0.18 & 9.75 \\
\hline FloppyTail_NoFrags & CEN/FA & CenStdExt/ref2015 & $\mathrm{X}$ & $\mathrm{X}$ & $\mathrm{X}$ & $34.8+6.3$ & 0.12 & 0.18 & 9.00 \\
\hline FloppyTail-Relax & CEN/FA & CenStdExt/ref2015 & $\mathrm{X}$ & $\mathrm{X}$ & $\mathrm{X}$ & $27.7+8.1$ & 0.15 & 0.15 & 7.03 \\
\hline FloppyTail & CEN/FA & CenStdExt /ref2015 & $\mathrm{X}$ & $\mathrm{X}$ & $\mathrm{X}$ & $34.0+7.7$ & 0.11 & 0.18 & 7.94 \\
\hline FastFloppyTail-Relax & CEN/FA & CenStdExt /ref2015 & $\mathrm{X}$ & $\mathrm{X}$ & $\mathrm{X}$ & $31.0+9.0$ & 0.11 & 0.17 & 5.37 \\
\hline FastFloppyTail & CEN/FA & CenStdExt /ref2015 & $\mathrm{X}$ & $\mathrm{X}$ & $\mathrm{X}$ & $38.0+9.4$ & 0.12 & 0.20 & 10.1 \\
\hline FloppyTail_Rot & $\mathrm{CEN} / \mathrm{FA}$ & CenStdExt/ref2015 & $\mathrm{X}$ & $\mathrm{X}$ & $\mathrm{X}$ & $37.2+8.6$ & 0.12 & 0.19 & 10.4 \\
\hline FloppyTail_Rot-Relax & $\mathrm{CEN} / \mathrm{FA}$ & CenStdExt/ref2015 & $\mathrm{X}$ & $\mathrm{X}$ & $\mathrm{X}$ & $29.2+8.7$ & 0.13 & 0.15 & 6.25 \\
\hline \multicolumn{10}{|c|}{ AbInitio } \\
\hline AbInitio & CEN/FA & score0-3/ref2015 & $\mathrm{X}$ & $\mathrm{X}$ & $\mathrm{X}$ & $17.3+1.0$ & 0.32 & 0.31 & 12.2 \\
\hline AbInitioVO & $\mathrm{CEN} / \mathrm{FA}$ & score0-3/ref2015 & $\mathrm{X}$ & $\mathrm{X}$ & $\mathrm{X}$ & $22.7+2.6$ & 0.24 & 0.22 & 12.9 \\
\hline \multicolumn{10}{|c|}{ Robustelli et. al. } \\
\hline a99SB-disp & & Molecular Dynamics & & & & 36.73 & - & 0.17 & - \\
\hline
\end{tabular}


*Atom type abbreviations: $\mathrm{CEN}=$ centroid, $\mathrm{FA}=$ full-atom.

** $R_{g}$ values can be compared to experimentally determined values of $33.0 \pm 3.0 \AA$ and $26.6 \pm 0.5$ $\AA$ from SAXS ${ }^{19}$ and NMR ${ }^{20}$ data, respectively. EFRET RMSD values were computed from data from Ferrie et. al., Ferrie et. al. and Nath et. al. ${ }^{1,21-22}$. Distance RMSD values were computed from Grupi et. al. and Lee et al. ${ }^{23-24}$ PRE RMSD values were computed from data from Sung et. al. and Dedmon et. al. ${ }^{8-9}$. 
Table S3: Comparison of Simulated $\alpha$ S Ensembles to Chemical Shift and RDC Data.

\begin{tabular}{|c|c|c|c|c|c|c|c|}
\hline Simulation Name & $\mathbf{N}$ & H & C & $\mathrm{Ca}$ & $C \beta$ & $\begin{array}{l}\text { All } \\
\text { CS } \\
\end{array}$ & RDC \\
\hline VDW_PP & 1.19 & 0.88 & 0.84 & 0.76 & 1.05 & 0.87 & 0.55 \\
\hline VDW_PPFI & 1.52 & 0.17 & 0.55 & 0.65 & 1.06 & 0.91 & 0.55 \\
\hline VDW_PPFISC & 1.83 & 0.19 & 0.49 & 0.50 & 1.08 & 1.01 & 0.52 \\
\hline CenStd_PP & 3.42 & 0.25 & 0.70 & 0.65 & 1.28 & 1.69 & 0.55 \\
\hline CenStd_PPFI & 3.66 & 0.20 & 0.66 & 1.07 & 1.31 & 1.83 & 0.44 \\
\hline CenNath & 2.10 & 0.22 & 0.40 & 0.50 & 1.37 & 1.16 & 0.51 \\
\hline CenStd_Ext_PP & 2.07 & 0.22 & 0.40 & 0.49 & 1.35 & 1.15 & 0.51 \\
\hline CenStd_Ext_PPFI & 3.60 & 0.28 & 1.28 & 1.52 & 1.42 & 1.95 & 0.36 \\
\hline Beta_PP & 0.95 & 1.06 & 0.57 & 2.67 & 0.14 & 1.38 & 0.54 \\
\hline Beta_PPFI & 3.37 & 0.31 & 1.18 & 1.58 & 1.18 & 1.83 & 0.39 \\
\hline Beta_PPFISC & 2.93 & 0.26 & 1.68 & 2.60 & 1.23 & 1.99 & 0.32 \\
\hline Beta_PPSC & 1.59 & 0.13 & 1.00 & 0.41 & 1.11 & 0.99 & 0.57 \\
\hline SimAnn_PP & 2.74 & 0.14 & 0.65 & 0.49 & 1.06 & 1.37 & 0.52 \\
\hline SimAnn_PPFI & 3.63 & 0.31 & 0.83 & 1.10 & 1.14 & 1.81 & 0.43 \\
\hline SimAnn_PPFISC & 3.10 & 0.27 & 1.20 & 1.81 & 1.08 & 1.77 & 0.37 \\
\hline SimAnn_PPSC & 2.17 & 0.12 & 0.64 & 0.43 & 1.03 & 1.13 & 0.51 \\
\hline FloppyTail_score12 & 2.03 & 0.18 & 0.39 & 0.58 & 1.02 & 1.07 & 0.48 \\
\hline FloppyTail_ref2015 & 1.93 & 0.17 & 0.39 & 0.58 & 1.02 & 1.02 & 0.48 \\
\hline FloppyTail_Quota & 1.75 & 0.18 & 0.38 & 0.46 & 1.03 & 0.95 & 0.49 \\
\hline FloppyTail_Loops & 1.52 & 0.13 & 0.40 & 0.39 & 1.02 & 0.86 & 0.55 \\
\hline FloppyTail_NoFrags & 1.52 & 0.13 & 0.41 & 0.38 & 1.01 & 0.86 & 0.55 \\
\hline FloppyTail-Relax & 1.79 & 0.13 & 0.38 & 0.34 & 0.98 & 0.94 & 0.44 \\
\hline FloppyTail & 1.69 & 0.13 & 0.36 & 0.35 & 0.98 & 0.90 & 0.44 \\
\hline FastFloppyTail-Relax & 1.62 & 0.11 & 0.47 & 0.36 & 1.02 & 0.90 & 0.42 \\
\hline FastFloppyTail & 1.55 & 0.12 & 0.42 & 0.33 & 1.01 & 0.86 & 0.41 \\
\hline FloppyTail_Rot & 1.66 & 0.13 & 0.36 & 0.33 & 0.98 & 0.89 & 0.44 \\
\hline FloppyTail_Rot-Relax & 1.77 & 0.12 & 0.38 & 0.34 & 0.97 & 0.93 & 0.44 \\
\hline AbInitio & 2.68 & 0.25 & 1.37 & 2.08 & 1.23 & 1.73 & 0.38 \\
\hline AbInitioVO & 2.24 & 0.24 & 0.61 & 0.72 & 1.03 & 1.19 & 0.47 \\
\hline$B$ & 1.46 & 0.14 & 0.31 & 0.51 & 1.04 & 0.85 & 0.41 \\
\hline
\end{tabular}

*All chemical shift data are reported as RMSD values from computed from data from Sung et al. ${ }^{8}$. RDC values are computed as Q-values, as described in Zweckstetter et. al. ${ }^{12}$, based on data from Bertoncini et al. (RDC) ${ }^{25}$. 
Table S4: Comparison of Simulated $\alpha$ S Ensembles to $J$-Coupling Data.

\begin{tabular}{|c|c|c|c|c|c|c|}
\hline Simulation Name & ${ }^{3} \boldsymbol{J}_{\mathrm{HNH} \boldsymbol{\alpha}}$ & ${ }^{1} J_{\mathrm{C} a \mathrm{H} \alpha}$ & ${ }^{1} J_{\mathrm{NC} a}$ & ${ }^{2} J_{\mathrm{NC} a}$ & ${ }^{3} \boldsymbol{J}_{\mathbf{C}^{\prime} \mathbf{C}^{\prime}}$ & All $J$ \\
\hline \multicolumn{7}{|c|}{ Generalized Simulation } \\
\hline VDW_PP & 2.38 & 1.31 & 0.63 & 0.42 & 0.69 & 1.30 \\
\hline VDW_PPFI & 2.63 & 1.10 & 0.72 & 0.74 & 0.57 & 1.38 \\
\hline VDW_PPFISC & 2.39 & 1.51 & 0.94 & 0.93 & 0.52 & 1.42 \\
\hline CenStd_PP & 1.41 & 1.37 & 0.66 & 0.46 & 0.61 & 0.99 \\
\hline CenStd_PPFI & 1.21 & 1.68 & 0.98 & 1.25 & 0.14 & 1.17 \\
\hline CenNath & 1.67 & 1.46 & 0.25 & 0.45 & 0.41 & 1.03 \\
\hline CenStd_Ext_PP & 1.61 & 1.43 & 0.25 & 0.45 & 0.39 & 1.00 \\
\hline CenStd_Ext_PPFI & 1.85 & 3.15 & 0.89 & 1.55 & 0.42 & 1.83 \\
\hline Beta_PP & 0.89 & 1.39 & 0.43 & 0.40 & 0.73 & 0.85 \\
\hline Beta_PPFI & 1.22 & 2.81 & 0.91 & 1.29 & 0.38 & 1.55 \\
\hline Beta_PPFISC & 2.41 & 4.31 & 1.15 & 1.48 & 0.40 & 2.37 \\
\hline Beta_PPSC & 0.82 & 1.28 & 0.38 & 0.40 & 0.74 & 0.79 \\
\hline SimAnn_PP & 0.67 & 1.68 & 0.55 & 0.45 & 0.58 & 0.91 \\
\hline SimAnn_PPFI & 0.84 & 2.08 & 0.91 & 1.37 & 0.24 & 1.25 \\
\hline SimAnn_PPFISC & 1.50 & 3.21 & 0.99 & 1.39 & 0.34 & 1.77 \\
\hline SimAnn_PPSC & 0.43 & 1.39 & 0.60 & 0.49 & 0.50 & 0.76 \\
\hline \multicolumn{7}{|c|}{ FloppyTail } \\
\hline FloppyTail_score12 & 0.48 & 1.64 & 0.54 & 0.70 & 0.16 & 0.88 \\
\hline FloppyTail_ref2015 & 0.52 & 1.68 & 0.52 & 0.79 & 0.17 & 0.88 \\
\hline FloppyTail_Quota & 0.62 & 1.55 & 0.49 & 0.74 & 0.19 & 0.85 \\
\hline FloppyTail_Loops & 0.51 & 1.10 & 0.35 & 0.43 & 0.10 & 0.60 \\
\hline FloppyTail_NoFrags & 0.53 & 1.11 & 0.35 & 0.43 & 0.10 & 0.60 \\
\hline FloppyTail-Relax & 0.59 & 1.25 & 0.41 & 0.54 & 0.16 & 0.69 \\
\hline FloppyTail & 0.64 & 1.45 & 0.40 & 0.53 & 0.16 & 0.77 \\
\hline FastFloppyTail-Relax & 0.65 & 1.14 & 0.36 & 0.39 & 0.26 & 0.64 \\
\hline FastFloppyTail & 0.56 & 1.22 & 0.35 & 0.39 & 0.24 & 0.65 \\
\hline FloppyTail_Rot & 0.61 & 1.41 & 0.40 & 0.51 & 0.16 & 0.75 \\
\hline FloppyTail_Rot-Relax & 0.60 & 1.23 & 0.40 & 0.52 & 0.17 & 0.68 \\
\hline \multicolumn{7}{|c|}{ AbInitio } \\
\hline AbInitio & 2.15 & 3.49 & 0.99 & 1.32 & 0.37 & 1.98 \\
\hline AbInitioVO & 1.16 & 1.82 & 0.71 & 1.12 & 0.25 & 1.13 \\
\hline \multicolumn{7}{|c|}{ Robustelli et. al } \\
\hline a99SB-disp & 1.11 & - & - & - & 0.18 & - \\
\hline
\end{tabular}

*All data are reported as RMSD values computed from data from Mantsyzov et al. and Lee et. al. ${ }^{13,18}$. 


\section{Comparison of $\alpha$ S Per Residue Percent Helicity}
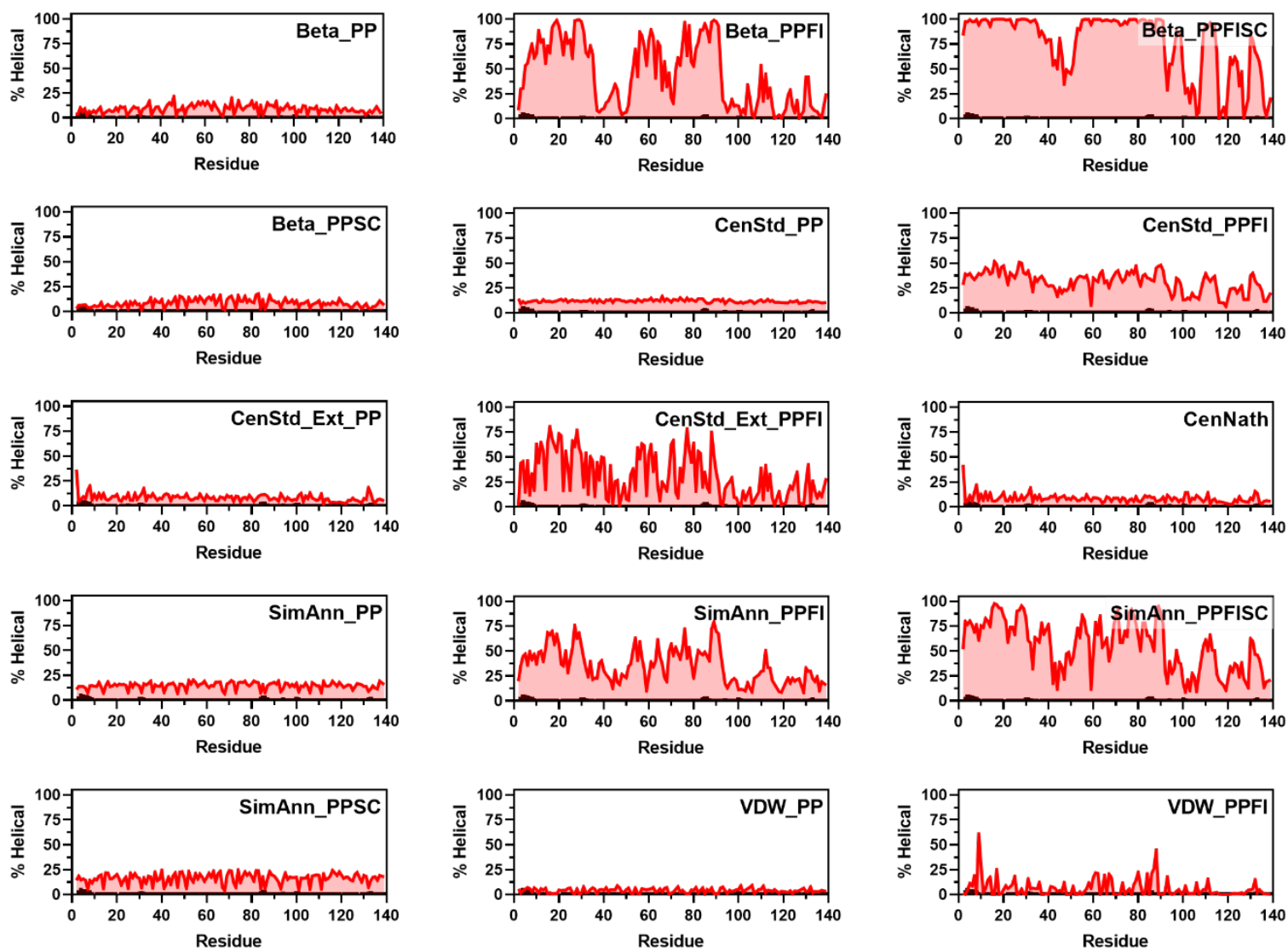

Figure S1: Plots of Percent Helicity. Plots of the percentage of structures containing helices on a per residue basis from Beta_PP (Row 1 Left), Beta_PPFI (Row 1 Middle), Beta_PPFISC (Row 1 Right), Beta_PPSC (Row 2 Left), CenStd_PP (Row 2 Middle), CenStd_PPFI (Row 2 Right), CenStd_Ext_PP (Row 3 Left), CenStd_Ext_PPFI (Row 3 Middle), CenNath (Row 3 Right), SimAnn_PP (Row 4 Left), SimAnn_PPFI (Row 4 Middle), SimAnn_PPFISC (Row 4 Right), SimAnn_PPSC (Row 5 Left), VDW_PP (Row 5 Middle) and VDW_PPFI (Row 5 Right) ensembles compared to percentages computed from Sung et al. chemical shift data using the D2D method from Camilloni et al. ${ }^{8,26}$. 

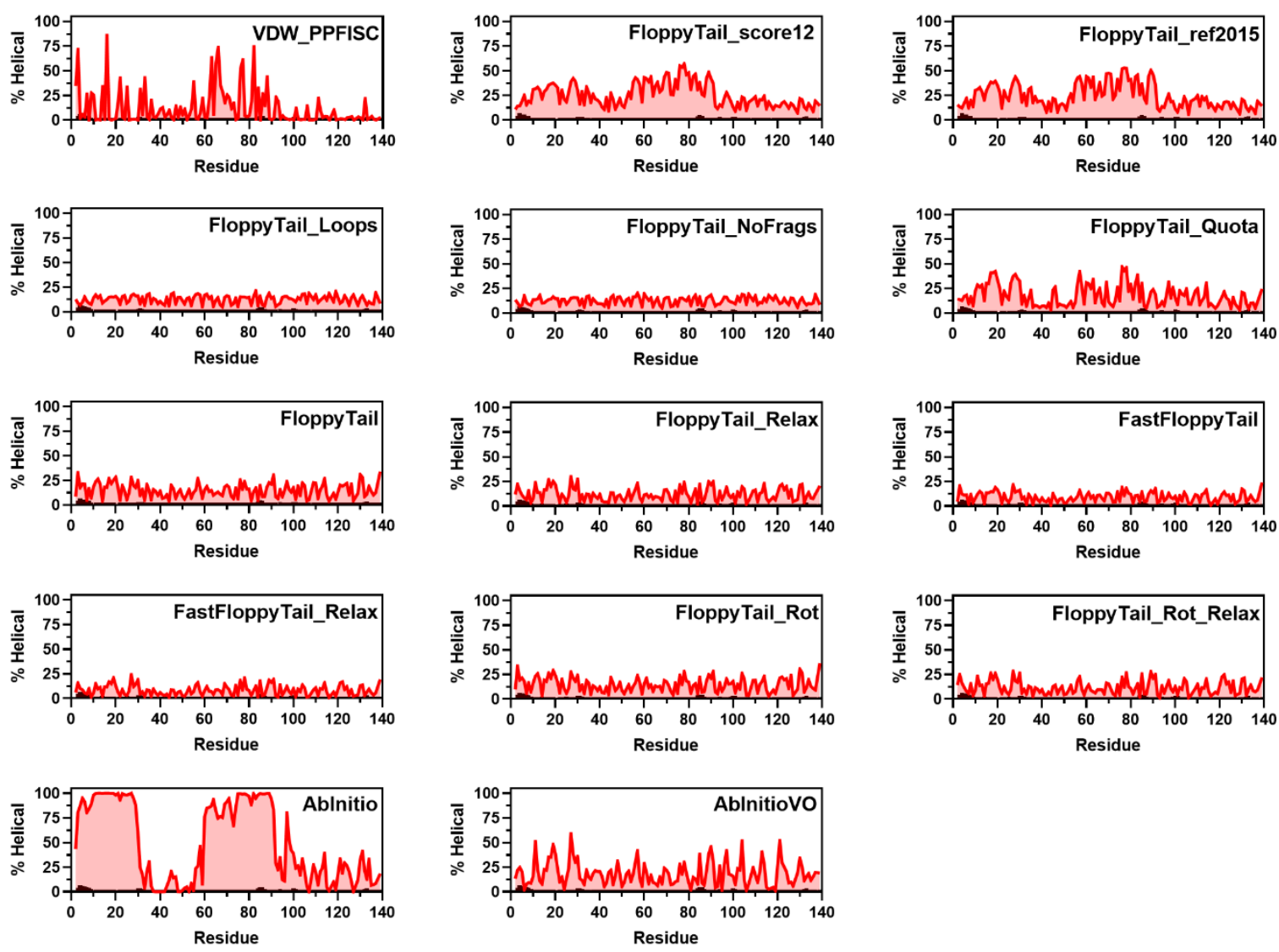

Figure S2: Plots of Percent Helicity. Plots of the percentage of structures containing helices on a per residue basis from VDW_PPFISC (Row 1 Left), FloppyTail_score12 (Row 1 Middle), FloppyTail_ref2015 (Row 1 Right), FloppyTail_Quota (Row 2 Left), FloppyTail_NoFrags (Row 2 Middle), FloppyTail_Loops (Row 2 Right), FloppyTail (Row 3 Left), FloppyTail_Relax (Row 3 Middle), FastFloppyTail (Row 3 Right), FastFloppyTail_Relax (Row 4 Left), FloppyTail_Rot (Row 4 Middle), FloppyTail_Rot_Relax (Row 4 Right), AbInitio (Row 5 Left) and AbInitioVO (Row 5 Middle) ensembles compared to percentages computed from Sung et al. chemical shift data using the D2D method from Camilloni et al. ${ }^{8,26}$. 


\section{Comparison with $\alpha \mathrm{S}$ Radius of Gyration Data}
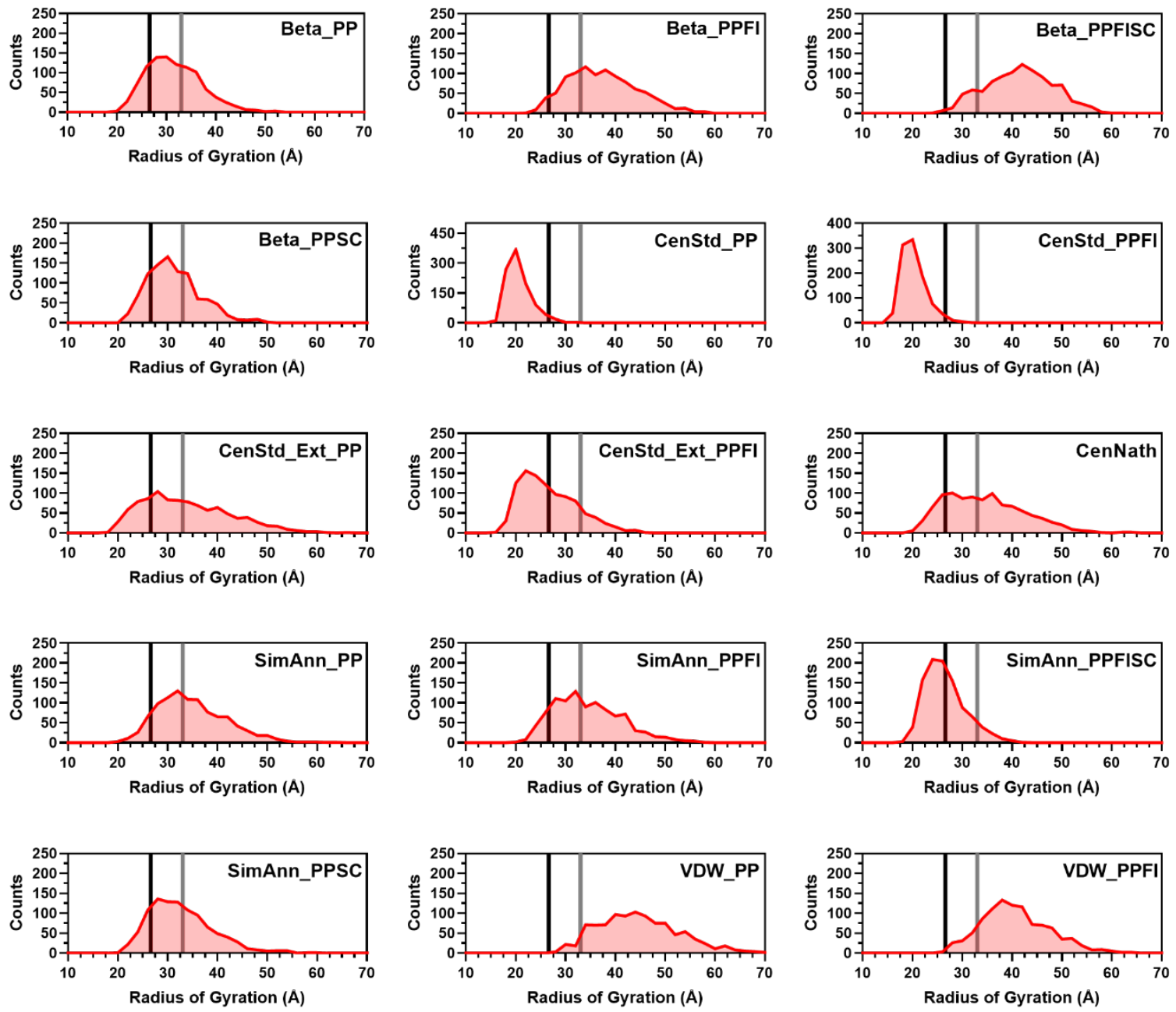

Figure S3: Histograms of Radii of Gyration. Histograms of the radius of gyration from Beta_PP (Row 1 Left), Beta_PPFI (Row 1 Middle), Beta_PPFISC (Row 1 Right), Beta_PPSC (Row 2 Left), CenStd_PP (Row 2 Middle), CenStd_PPFI (Row 2 Right), CenStd_Ext_PP (Row 3 Left), CenStd_Ext_PPFI (Row 3 Middle), CenNath (Row 3 Right), SimAnn_PP (Row 4 Left), SimAnn_PPFI (Row 4 Middle), SimAnn_PPFISC (Row 4 Right), SimAnn_PPSC (Row 5 Left), VDW_PP (Row 5 Middle) and VDW_PPFI (Row 5 Right) ensembles compared to experimental values from $\mathrm{SAXS}^{19}$ (grey) and NMR ${ }^{20}$ (black). 

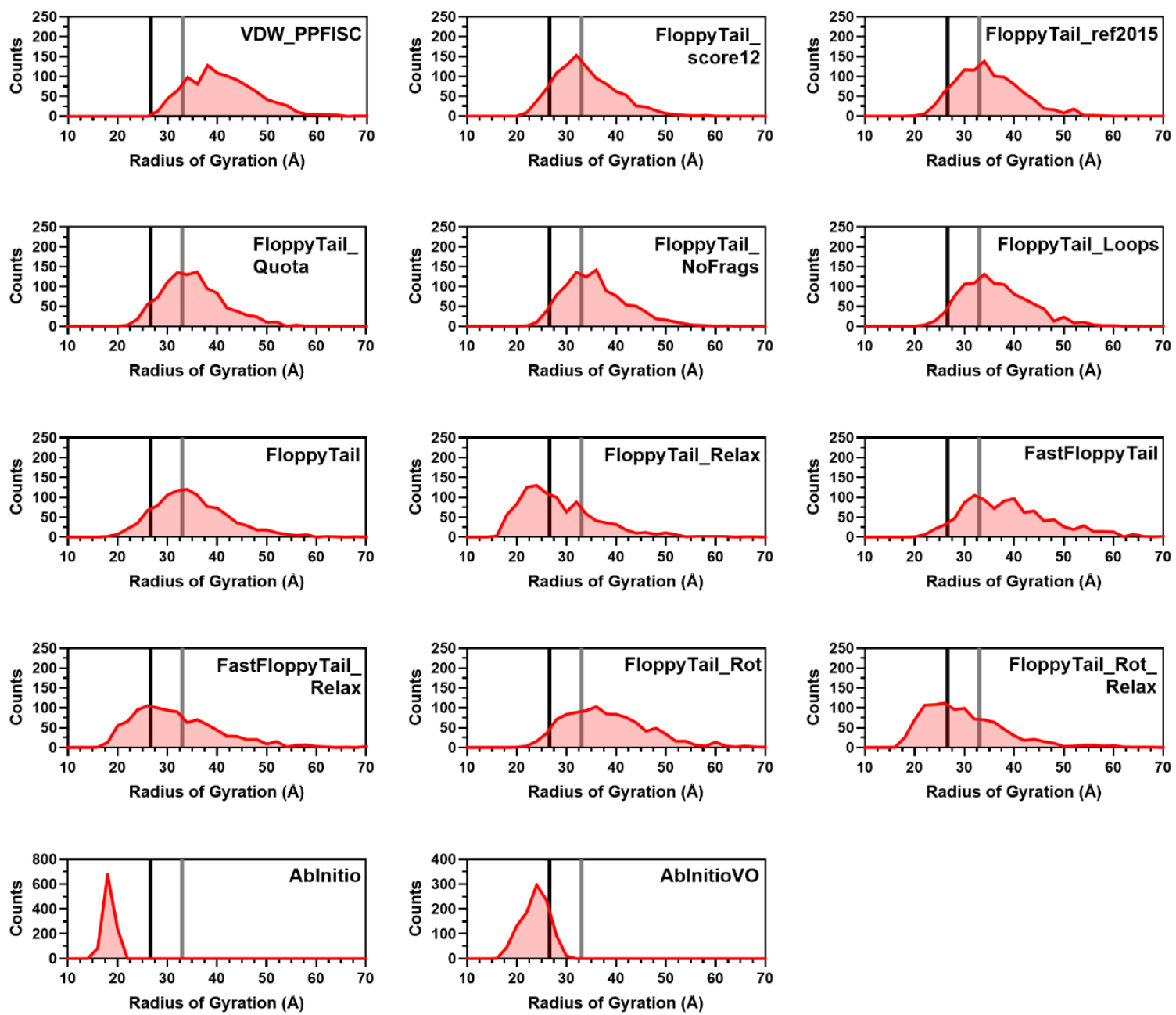

Figure S4: Histograms of Radii of Gyration. Histograms of the radius of gyration from VDW_PPFISC (Row 1 Left), FloppyTail_score12 (Row 1 Middle), FloppyTail_ref2015 (Row 1 Right), FloppyTail_Quota (Row 2 Left), FloppyTail_NoFrags (Row 2 Middle), FloppyTail_Loops (Row 2 Right), FloppyTail (Row 3 Left), FloppyTail_Relax (Row 3 Middle), FastFloppyTail (Row 3 Right), FastFloppyTail_Relax (Row 4 Left), FloppyTail_Rot (Row 4 Middle), FloppyTail_Rot_Relax (Row 4 Right), AbInitio (Row 5 Left) and AbInitioVO (Row 5 Middle) ensembles compared to experimental values from SAXS ${ }^{19}$ (grey) and NMR ${ }^{20}$ (black). 


\section{Comparison with $\alpha$ S FRET Data}
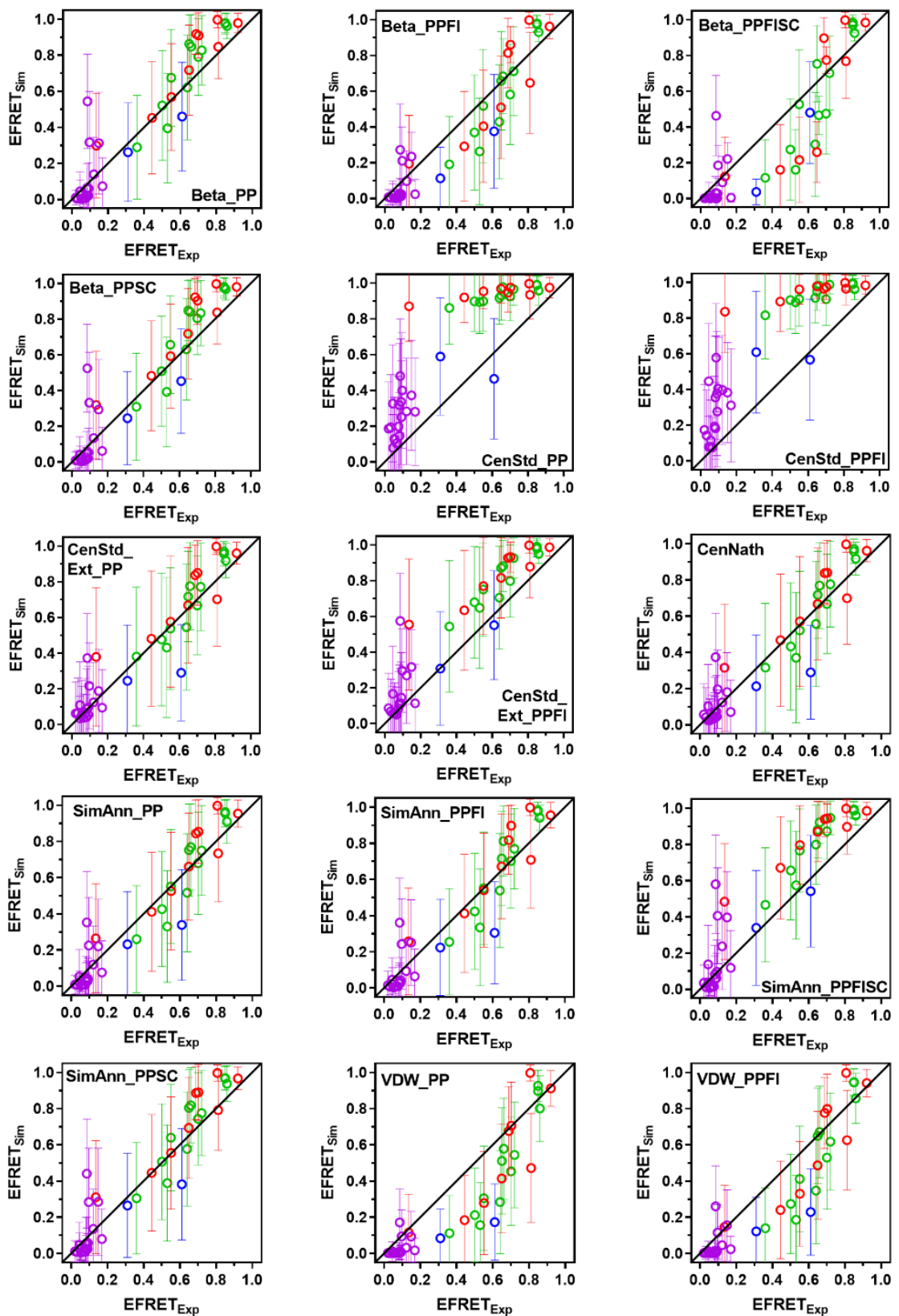

Figure S5: Comparison of Simulated EFret with Experimental EFret. Simulated EFrets for Beta_PP (Row 1 Left), Beta_PPFI (Row 1 Center), Beta_PPFISC (Row 1 Right), Beta_PPSC (Row 2 Left), CenStd_PP (Row 2 Center), CenStd_PPFI (Row 2 Right), CenStd_Ext_PP (Row 3 Left), CenStd_Ext_PPFI (Row 3 Center), CenNath (Row 3 Right), SimAnn_PP (Row 4 Left), SimAnn_PPFI (Row 4 Center), SimAnn_PPFISC (Row 4 Right), SimAnn_PPSC (Row 5 Left), VDW_PP (Row 5 Middle), VDW_PPFI (Row 5 Right) with data from Ferrie et al. Cnf-Trp ${ }^{21}$ (Purple) and Fam-Raz ${ }^{21}$ (Red) Pairs, Ferrie et al. Mcm-Acd pair ${ }^{22}$ (Blue), and Nath et al. ${ }^{1}$ (Green). 

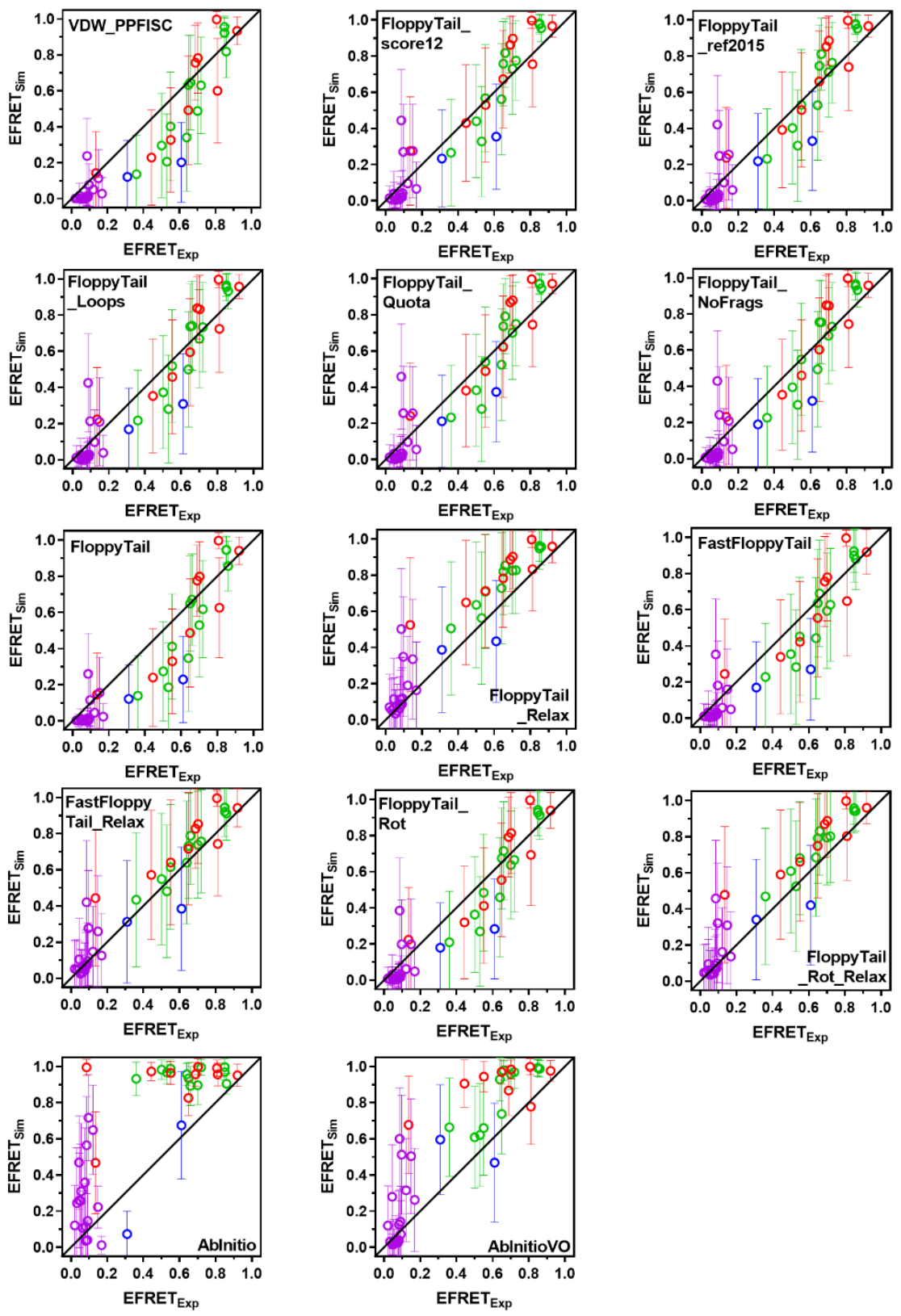

Figure S6: Comparison of Simulated EFRET with Experimental EFRET. Simulated E $_{\mathrm{FRET}}$ for VDW_PPFISC (Row 1 Left), FloppyTail_score12 (Row 1 Center), FloppyTail_ref2015 (Row 1 Right), FloppyTail_Loops (Row 2 Left), FloppyTail_Quota (Row 2 Center), FloppyTail_NoFrags (Row 2 Right), FloppyTail (Row 3 Left), FloppyTail_Relax (Row 3 Center), FastFloppyTail (Row 3 Right), FastFloppyTail_Relax (Row 4 Left), FloppyTail_Rot (Row 4 Center), FloppyTail_Rot_Relax (Row 4 Right), AbInitio (Row 5 Left) and AbInitioVO (Row 5 Middle) with data from Ferrie et al. Cnf-Trp ${ }^{21}$ (Purple) and Fam-Raz ${ }^{21}$ (Red) Pairs, Ferrie et al. Mcm-Acd pair $^{22}$ (Blue), and Nath et al. ${ }^{1}$ (Green). 


\section{Comparison with $\alpha$ S Distance Data}
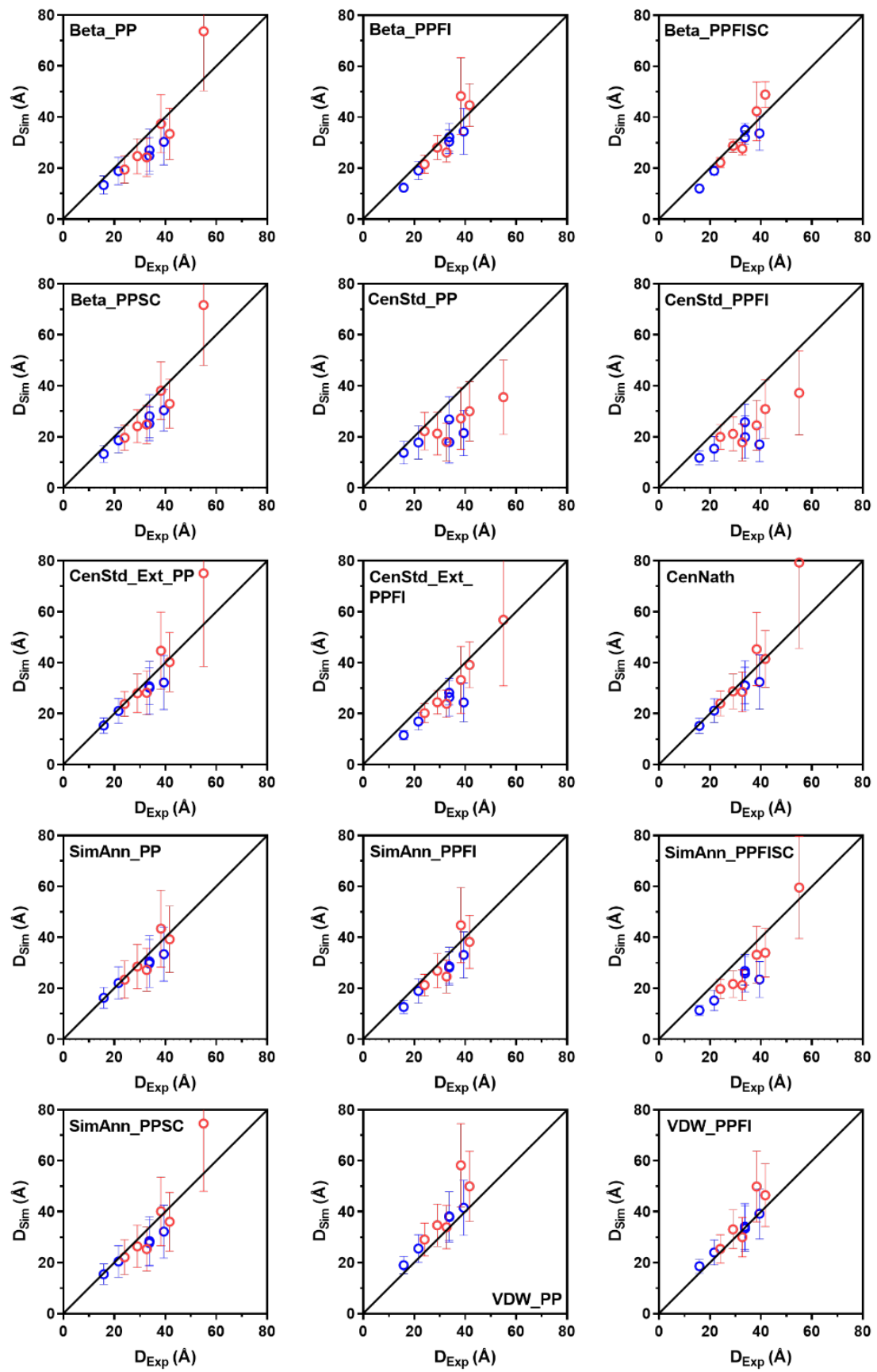

Figure S7: Comparison of Simulated Distance with Experimental Distances. Simulated distances for Beta_PP (Row 1 Left), Beta_PPFI (Row 1 Center), Beta_PPFISC (Row 1 Right), Beta_PPSC (Row 2 Left), CenStd_PP (Row 2 Center), CenStd_PPFI (Row 2 Right), CenStd_Ext_PP (Row 3 Left), CenStd_Ext_PPFI (Row 3 Center), CenNath (Row 3 Right), SimAnn_PP (Row 4 Left), SimAnn_PPFI (Row 4 Center), SimAnn_PPFISC (Row 4 Right), SimAnn_PPSC (Row 5 Left), VDW_PP (Row 5 Middle), VDW_PPFI (Row 5 Right) with data from Lee et al. ${ }^{24}$ (Red) and Grupi et al. ${ }^{23}$ (Blue). 

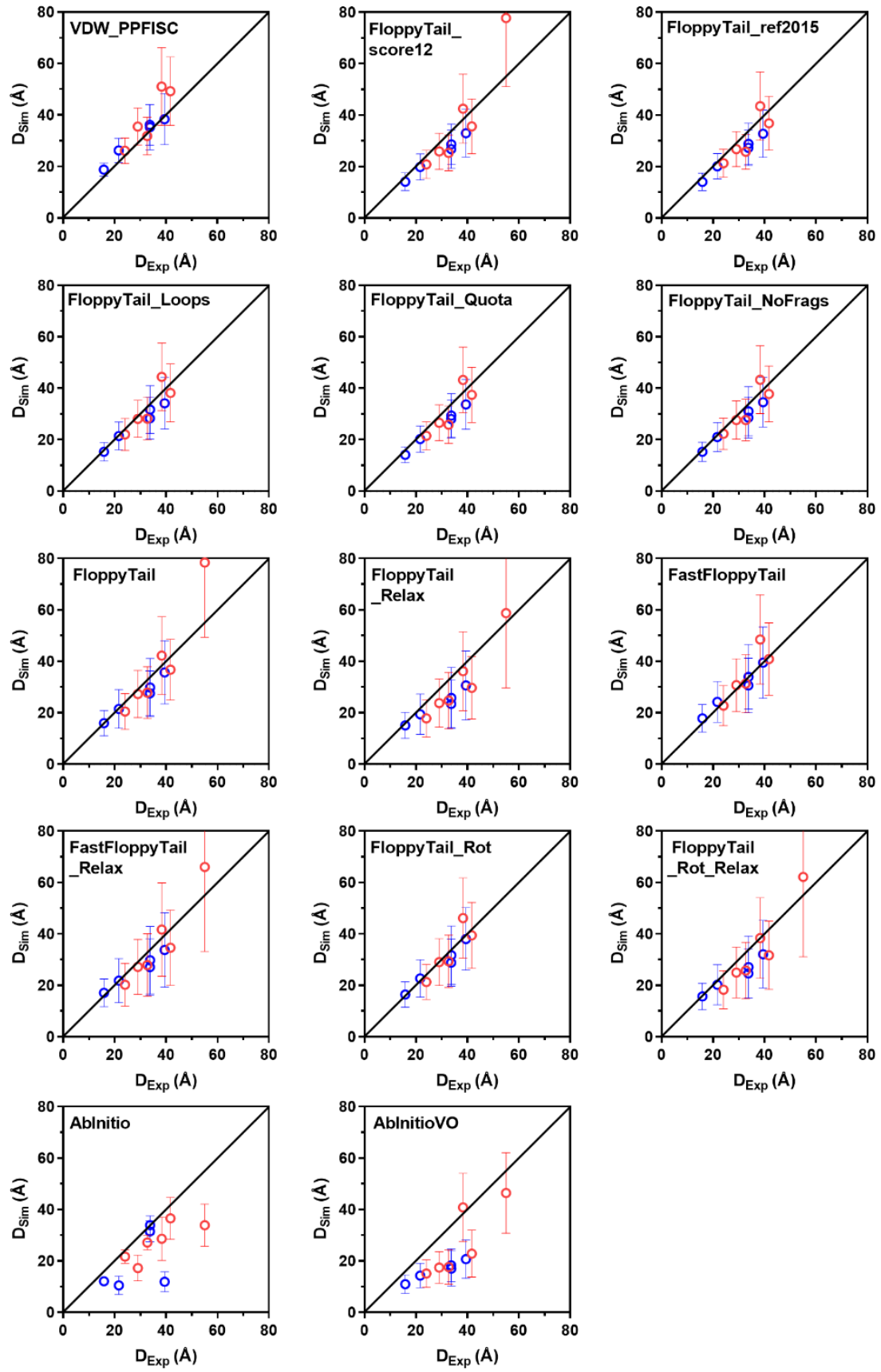

Figure S8: Comparison of Simulated Distance with Experimental Distances. Simulated distances for VDW_PPFISC (Row 1 Left), FloppyTail_score12 (Row 1 Center), FloppyTail_ref2015 (Row 1 Right), FloppyTail_Loops (Row 2 Left), FloppyTail_Quota (Row 2 Center), FloppyTail_NoFrags (Row 2 Right), FloppyTail (Row 3 Left), FloppyTail_Relax (Row 3 Center), FastFloppyTail (Row 3 Right), FastFloppyTail_Relax (Row 4 Left), FloppyTail_Rot (Row 4 Center), FloppyTail_Rot_Relax (Row 4 Right), AbInitio (Row 5 Left) and AbInitioVO (Row 5 Middle) with data from Lee et al. ${ }^{24}$ (Red) and Grupi et al. ${ }^{23}$ (Blue). 


\section{Comparison with $\alpha$ S PRE Data}

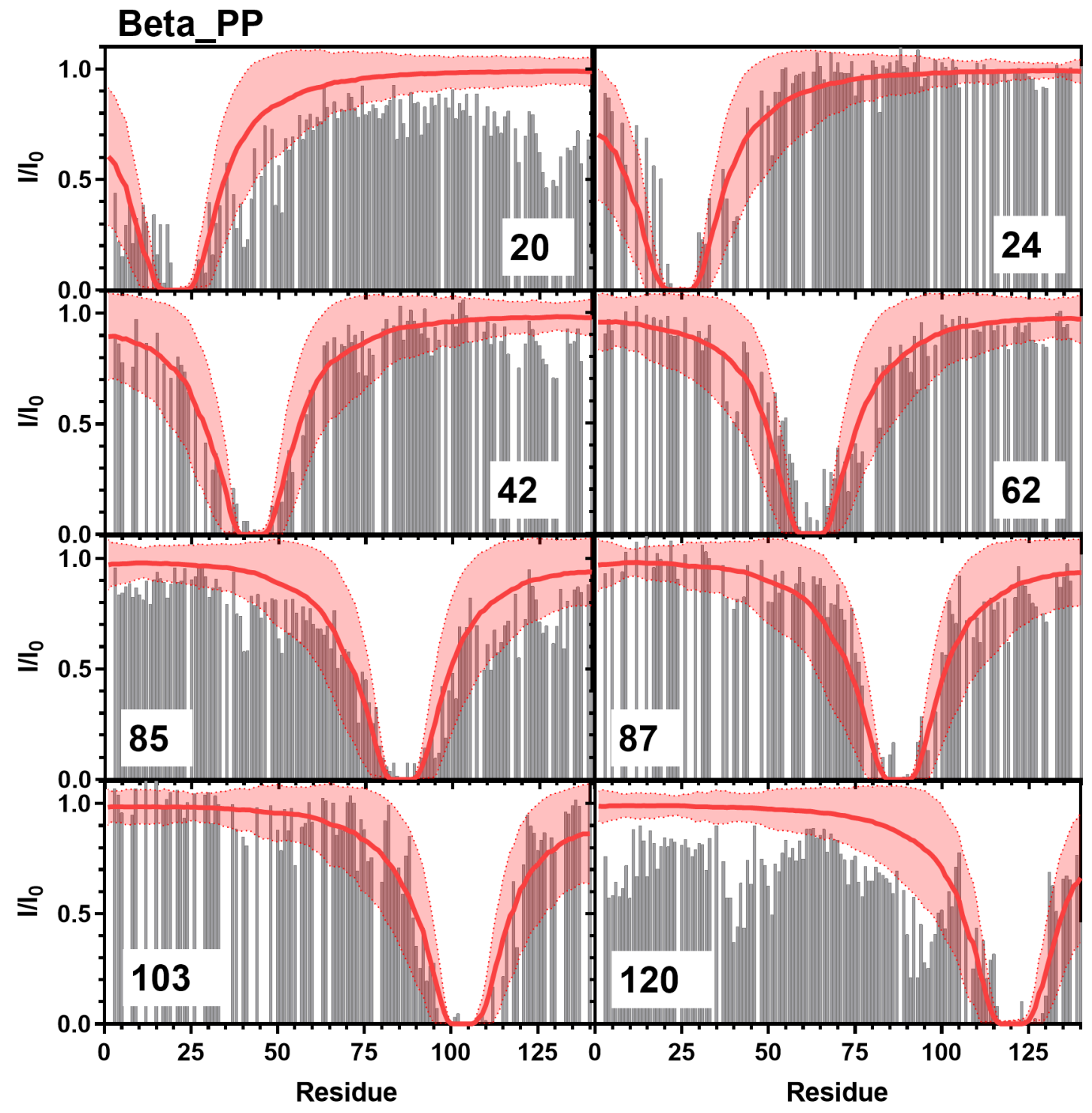

Figure S9: Comparison of Simulated PRE Values and Experimental PRE Values: Simulated PRE values from Beta_PP (red line) overlayed on top of experimental data (grey bars) from positions 20 (Top Left), $2 \overline{4}$ (Top Right), 42 (Upper Middle Left), 62 (Upper Middle Right), 85 (Lower Middle Left), 87 (Lower Middle Right), 103 (Bottom Left), 120 (Bottom Right). Experimental data for positions 20,85, and 120 are from Sung et al. ${ }^{8}$ and data for positions 24, 42, 62, 87, and 103 are from Dedmon et al. ${ }^{9}$. 


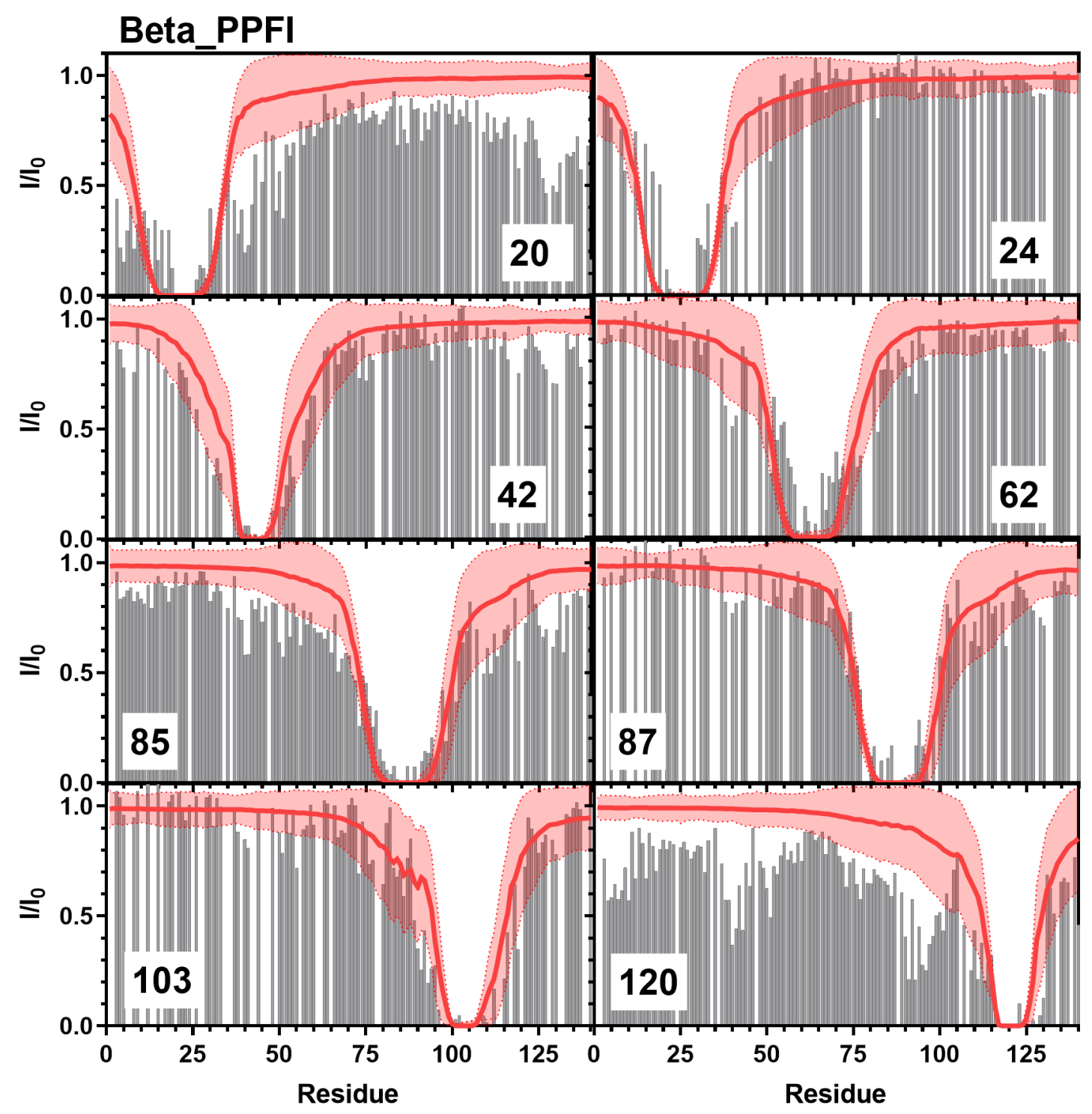

Figure S10: Comparison of Simulated PRE Values and Experimental PRE Values: Simulated PRE values from Beta_PPFI (red line) overlayed on top of experimental data (grey bars) from positions 20 (Top Left), 24 (Top Right), 42 (Upper Middle Left), 62 (Upper Middle Right), 85 (Lower Middle Left), 87 (Lower Middle Right), 103 (Bottom Left), 120 (Bottom Right). Experimental data for positions 20, 85, and 120 are from Sung et al. ${ }^{8}$ and data for positions $24,42,62,87$, and 103 are from Dedmon et al. ${ }^{9}$. 


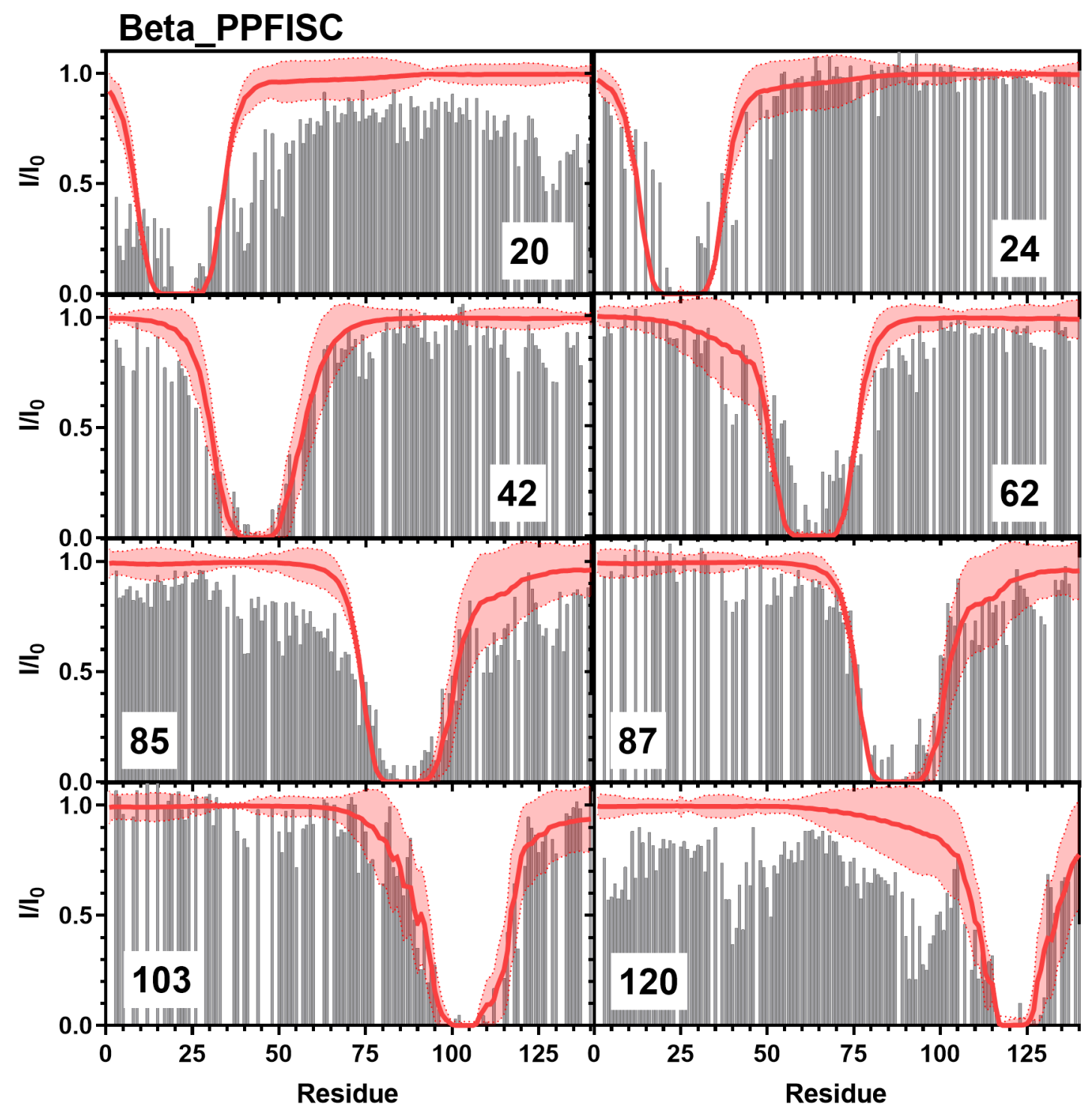

Figure S11: Comparison of Simulated PRE Values and Experimental PRE Values: Simulated PRE values from Beta_PPFISC (red line) overlayed on top of experimental data (grey bars) from positions 20 (Top Left), 24 (Top Right), 42 (Upper Middle Left), 62 (Upper Middle Right), 85 (Lower Middle Left), 87 (Lower Middle Right), 103 (Bottom Left), 120 (Bottom Right). Experimental data for positions 20, 85, and 120 are from Sung et al. ${ }^{8}$ and data for positions 24 , 42, 62, 87, and 103 are from Dedmon et al. ${ }^{9}$. 


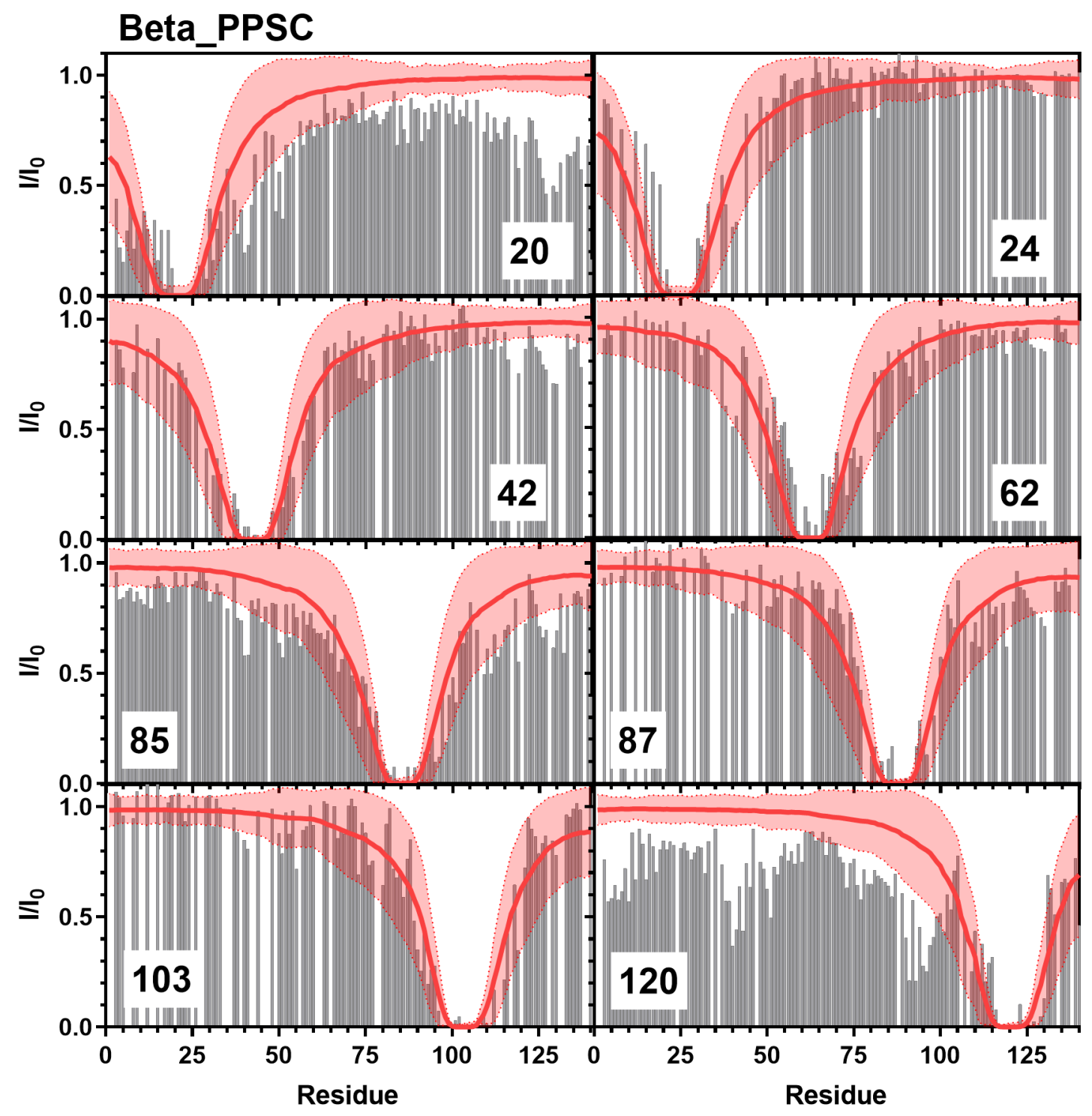

Figure S12: Comparison of Simulated PRE Values and Experimental PRE Values: Simulated PRE values from Beta_PPSC (red line) overlayed on top of experimental data (grey bars) from positions 20 (Top Left), 24 (Top Right), 42 (Upper Middle Left), 62 (Upper Middle Right), 85 (Lower Middle Left), 87 (Lower Middle Right), 103 (Bottom Left), 120 (Bottom Right). Experimental data for positions 20, 85, and 120 are from Sung et al. ${ }^{8}$ and data for positions $24,42,62,87$, and 103 are from Dedmon et al. ${ }^{9}$. 


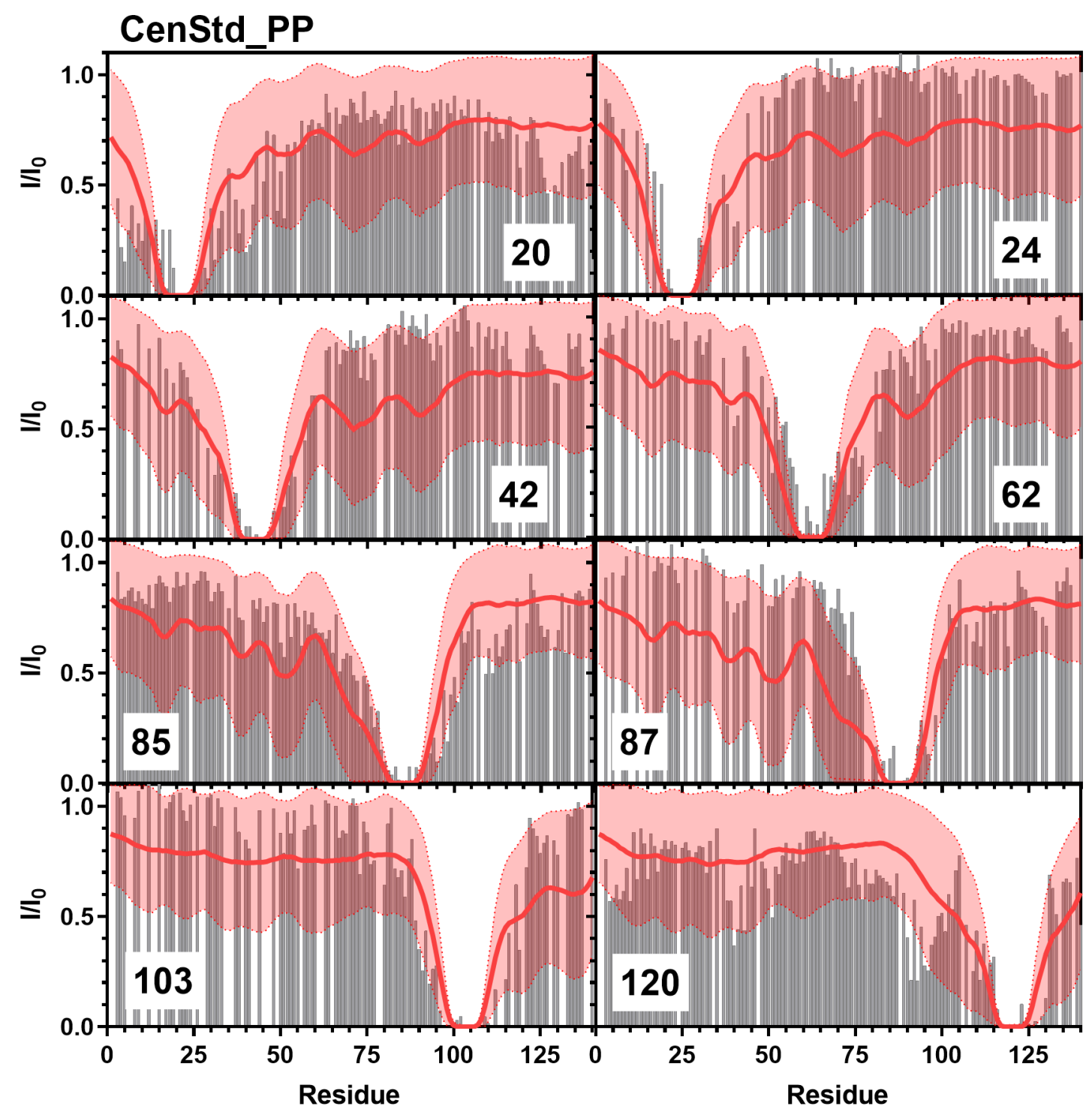

Figure S13: Comparison of Simulated PRE Values and Experimental PRE Values: Simulated PRE values from CenStd_PP (red line) overlayed on top of experimental data (grey bars) from positions 20 (Top Left), 24 (Top Right), 42 (Upper Middle Left), 62 (Upper Middle Right), 85 (Lower Middle Left), 87 (Lower Middle Right), 103 (Bottom Left), 120 (Bottom Right). Experimental data for positions 20, 85, and 120 are from Sung et al. ${ }^{8}$ and data for positions $24,42,62,87$, and 103 are from Dedmon et al. ${ }^{9}$. 


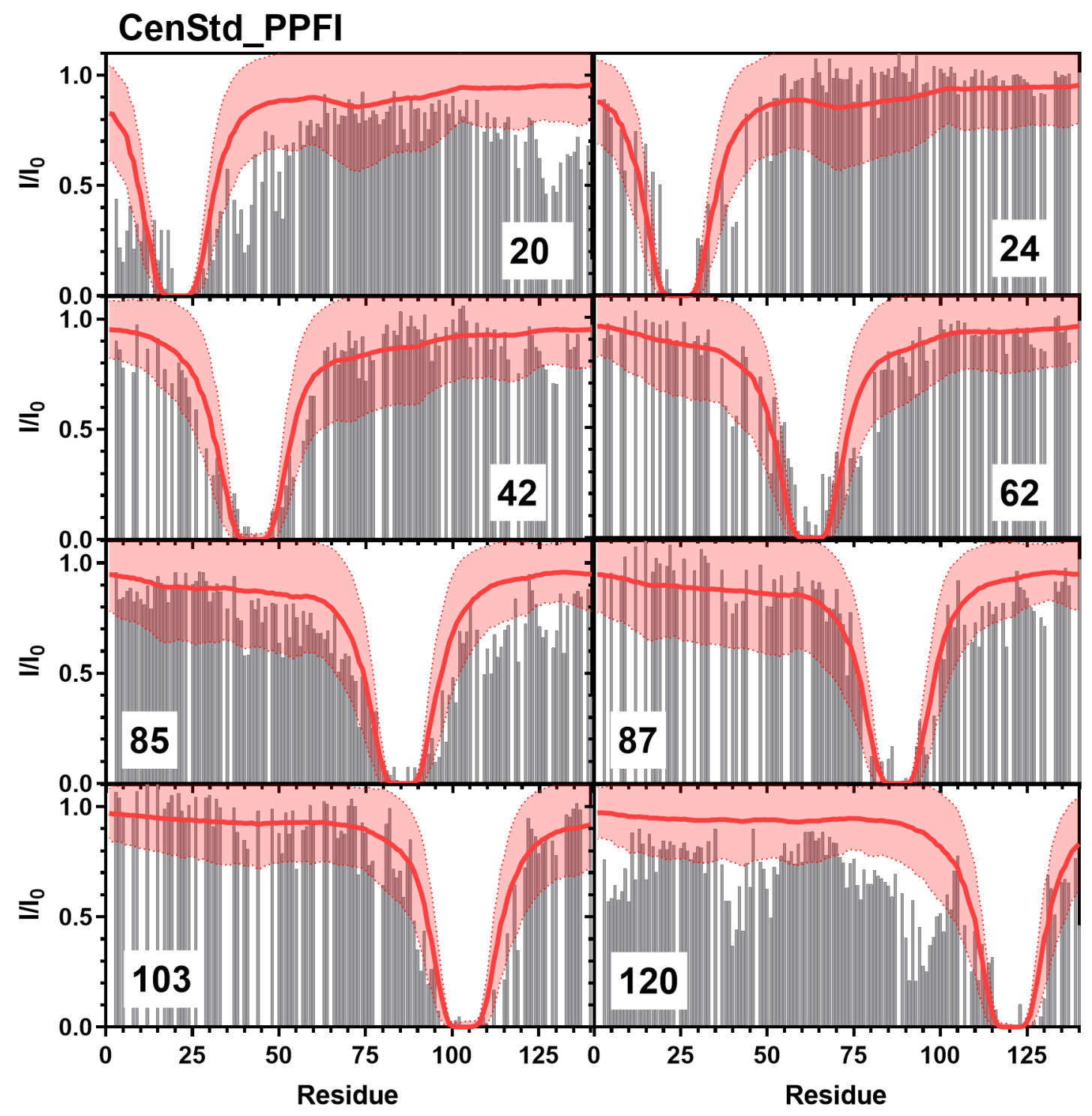

Figure S14: Comparison of Simulated PRE Values and Experimental PRE Values: Simulated PRE values from CenStd_PPFI (red line) overlayed on top of experimental data (grey bars) from positions 20 (Top Left), 24 (Top Right), 42 (Upper Middle Left), 62 (Upper Middle Right), 85 (Lower Middle Left), 87 (Lower Middle Right), 103 (Bottom Left), 120 (Bottom Right). Experimental data for positions 20, 85, and 120 are from Sung et al. ${ }^{8}$ and data for positions 24, 42, 62, 87, and 103 are from Dedmon et al. ${ }^{9}$. 


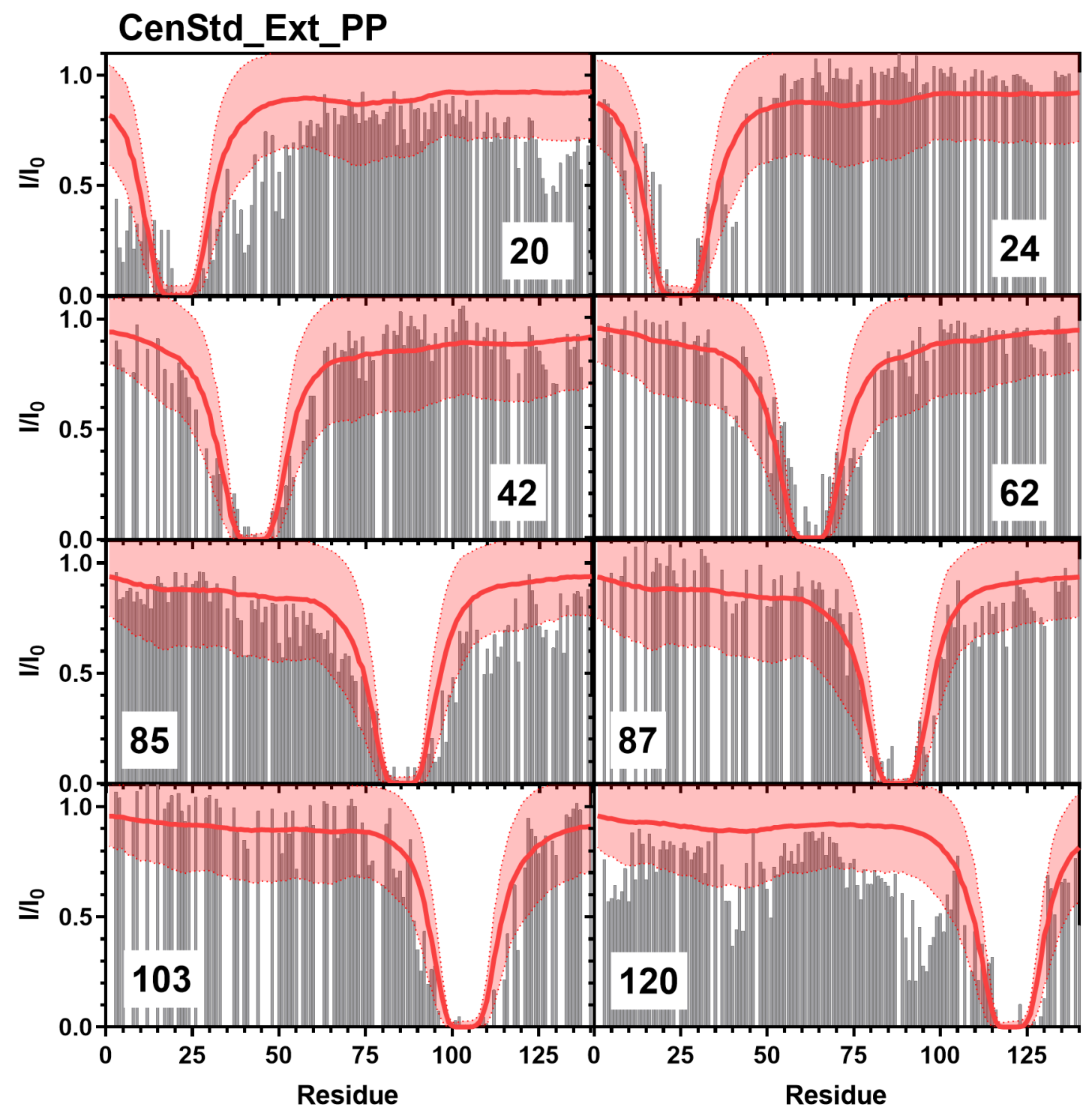

Figure S15: Comparison of Simulated PRE Values and Experimental PRE Values: Simulated PRE values from CenStd_Ext_PP (red line) overlayed on top of experimental data (grey bars) from positions 20 (Top Left), 24 (Top Right), 42 (Upper Middle Left), 62 (Upper Middle Right), 85 (Lower Middle Left), 87 (Lower Middle Right), 103 (Bottom Left), 120 (Bottom Right). Experimental data for positions 20, 85, and 120 are from Sung et al. ${ }^{8}$ and data for positions 24 , 42, 62, 87, and 103 are from Dedmon et al. ${ }^{9}$. 


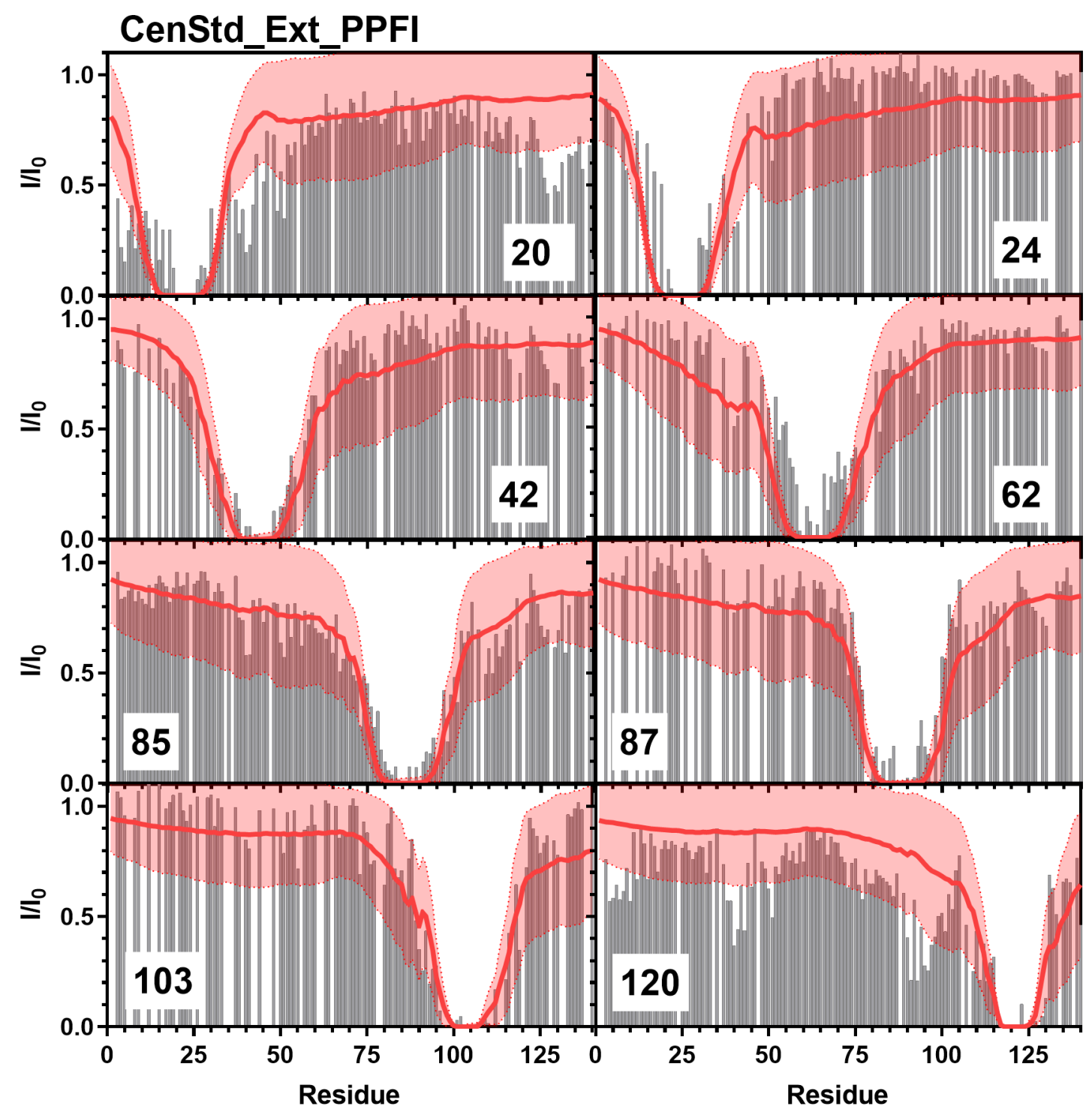

Figure S16: Comparison of Simulated PRE Values and Experimental PRE Values: Simulated PRE values from CenStd_Ext_PPFI (red line) overlayed on top of experimental data (grey bars) from positions 20 (Top Left), 24 (Top Right), 42 (Upper Middle Left), 62 (Upper Middle Right), 85 (Lower Middle Left), 87 (Lower Middle Right), 103 (Bottom Left), 120 (Bottom Right). Experimental data for positions 20, 85, and 120 are from Sung et al. ${ }^{8}$ and data for positions 24, 42, 62, 87, and 103 are from Dedmon et al. ${ }^{9}$. 


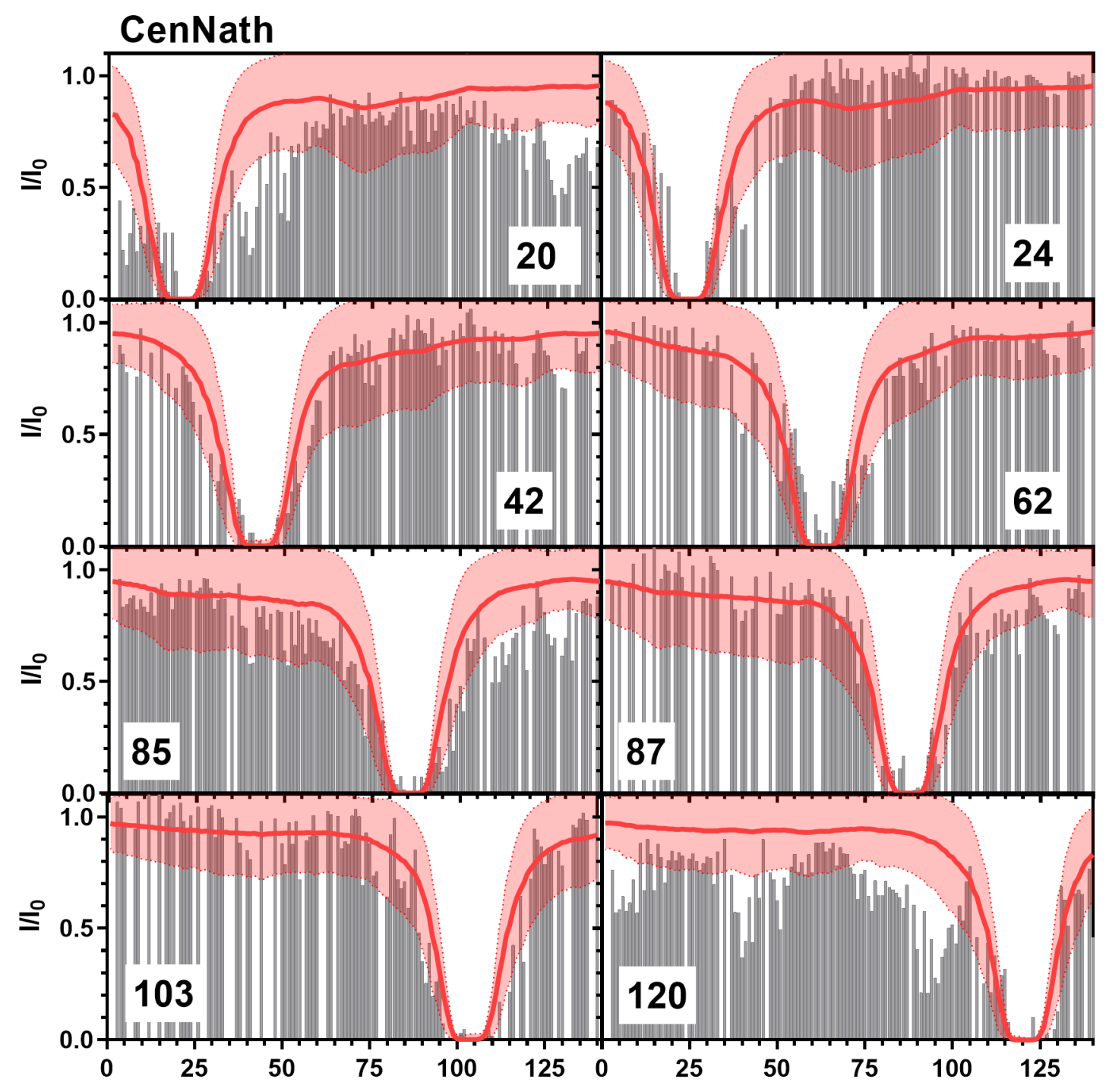

Figure S17: Comparison of Simulated PRE Values and Experimental PRE Values: Simulated PRE values from CenNath (red line) overlayed on top of experimental data (grey bars) from positions 20 (Top Left), 24 (Top Right), 42 (Upper Middle Left), 62 (Upper Middle Right), 85 (Lower Middle Left), 87 (Lower Middle Right), 103 (Bottom Left), 120 (Bottom Right). Experimental data for positions 20, 85, and 120 are from Sung et al. ${ }^{8}$ and data for positions $24,42,62,87$, and 103 are from Dedmon et al. ${ }^{9}$. 


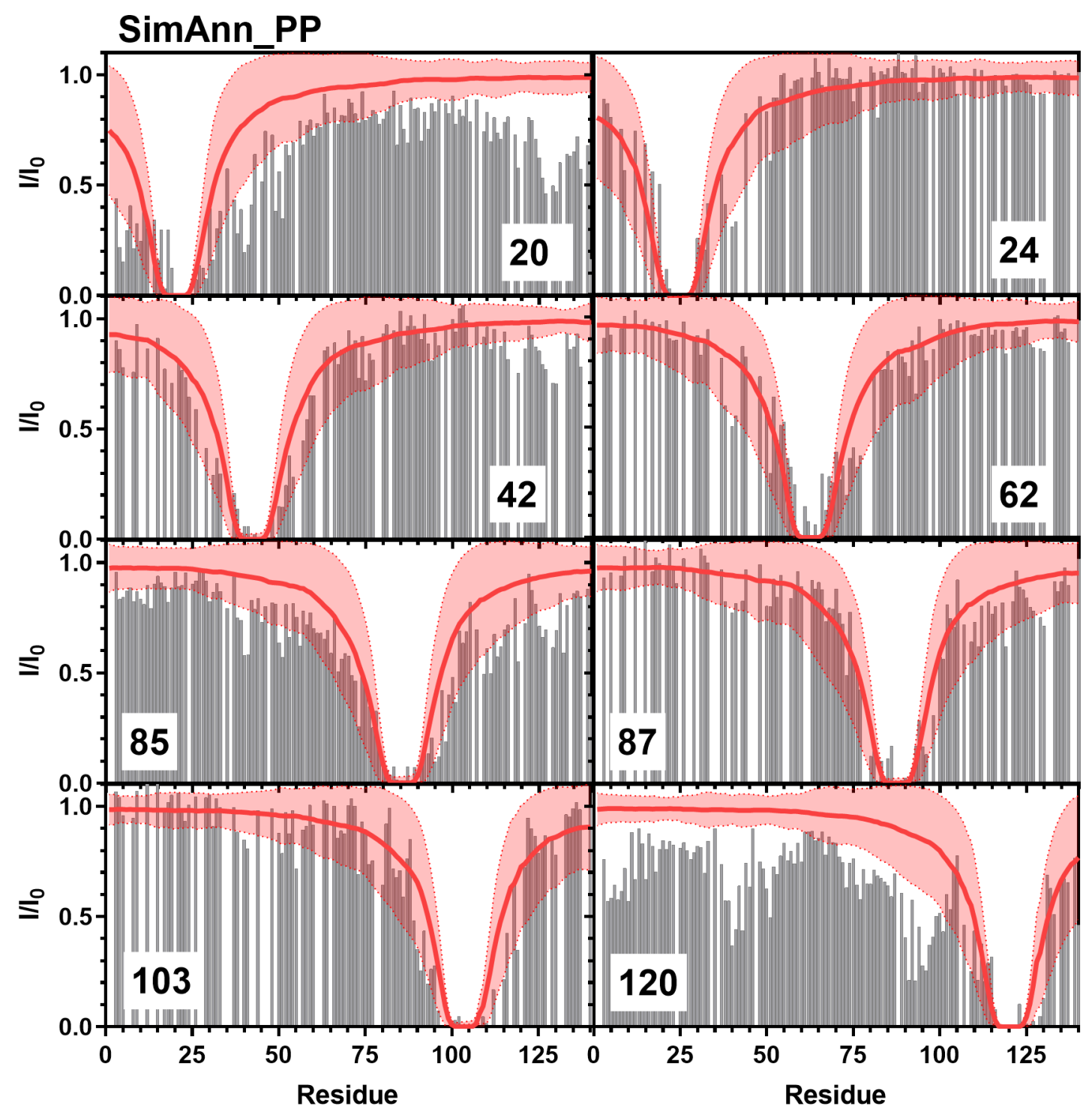

Figure S18: Comparison of Simulated PRE Values and Experimental PRE Values: Simulated PRE values from SimAnn_PP (red line) overlayed on top of experimental data (grey bars) from positions 20 (Top Left), 24 (Top Right), 42 (Upper Middle Left), 62 (Upper Middle Right), 85 (Lower Middle Left), 87 (Lower Middle Right), 103 (Bottom Left), 120 (Bottom Right). Experimental data for positions 20, 85, and 120 are from Sung et al. ${ }^{8}$ and data for positions 24 , 42, 62, 87, and 103 are from Dedmon et al. ${ }^{9}$. 


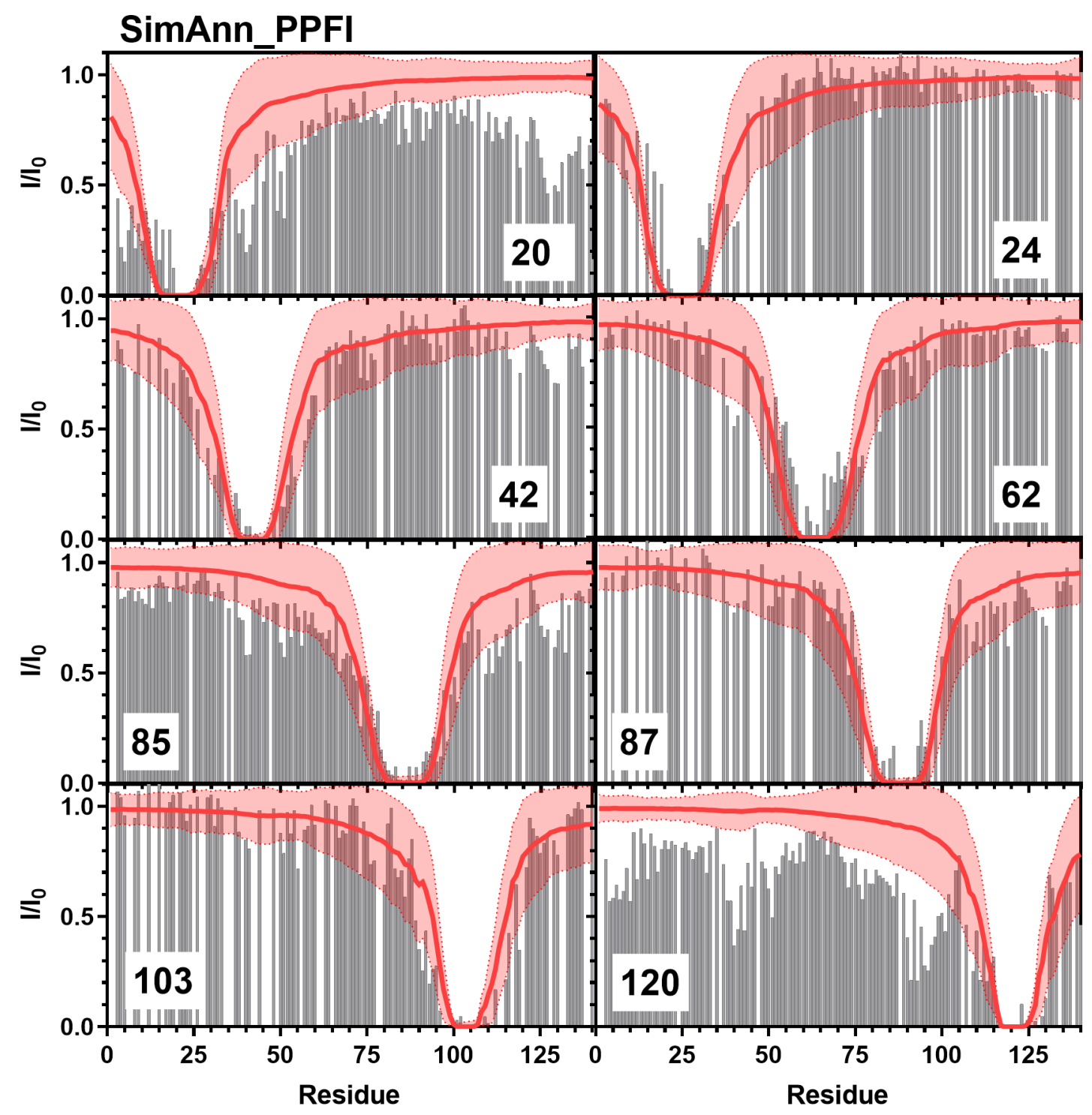

Figure S19: Comparison of Simulated PRE Values and Experimental PRE Values: Simulated PRE values from SimAnn_PPFI (red line) overlayed on top of experimental data (grey bars) from positions 20 (Top Left), 24 (Top Right), 42 (Upper Middle Left), 62 (Upper Middle Right), 85 (Lower Middle Left), 87 (Lower Middle Right), 103 (Bottom Left), 120 (Bottom Right). Experimental data for positions 20, 85, and 120 are from Sung et al. ${ }^{8}$ and data for positions 24 , 42, 62, 87, and 103 are from Dedmon et al. ${ }^{9}$. 


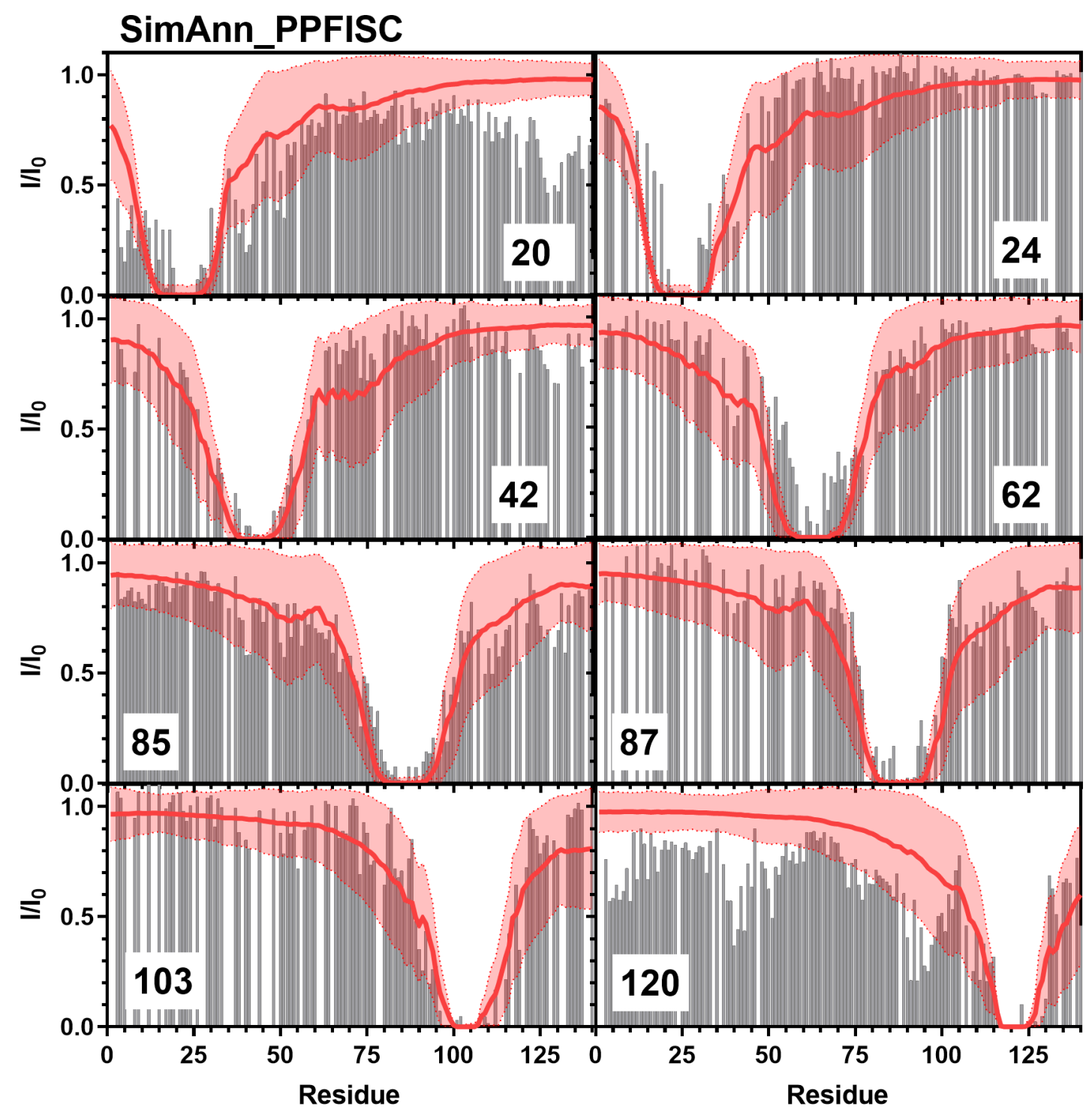

Figure S20: Comparison of Simulated PRE Values and Experimental PRE Values: Simulated PRE values from SimAnn_PPFISC (red line) overlayed on top of experimental data (grey bars) from positions 20 (Top Left), 24 (Top Right), 42 (Upper Middle Left), 62 (Upper Middle Right), 85 (Lower Middle Left), 87 (Lower Middle Right), 103 (Bottom Left), 120 (Bottom Right). Experimental data for positions 20, 85, and 120 are from Sung et al. ${ }^{8}$ and data for positions 24 , 42, 62, 87, and 103 are from Dedmon et al. ${ }^{9}$. 


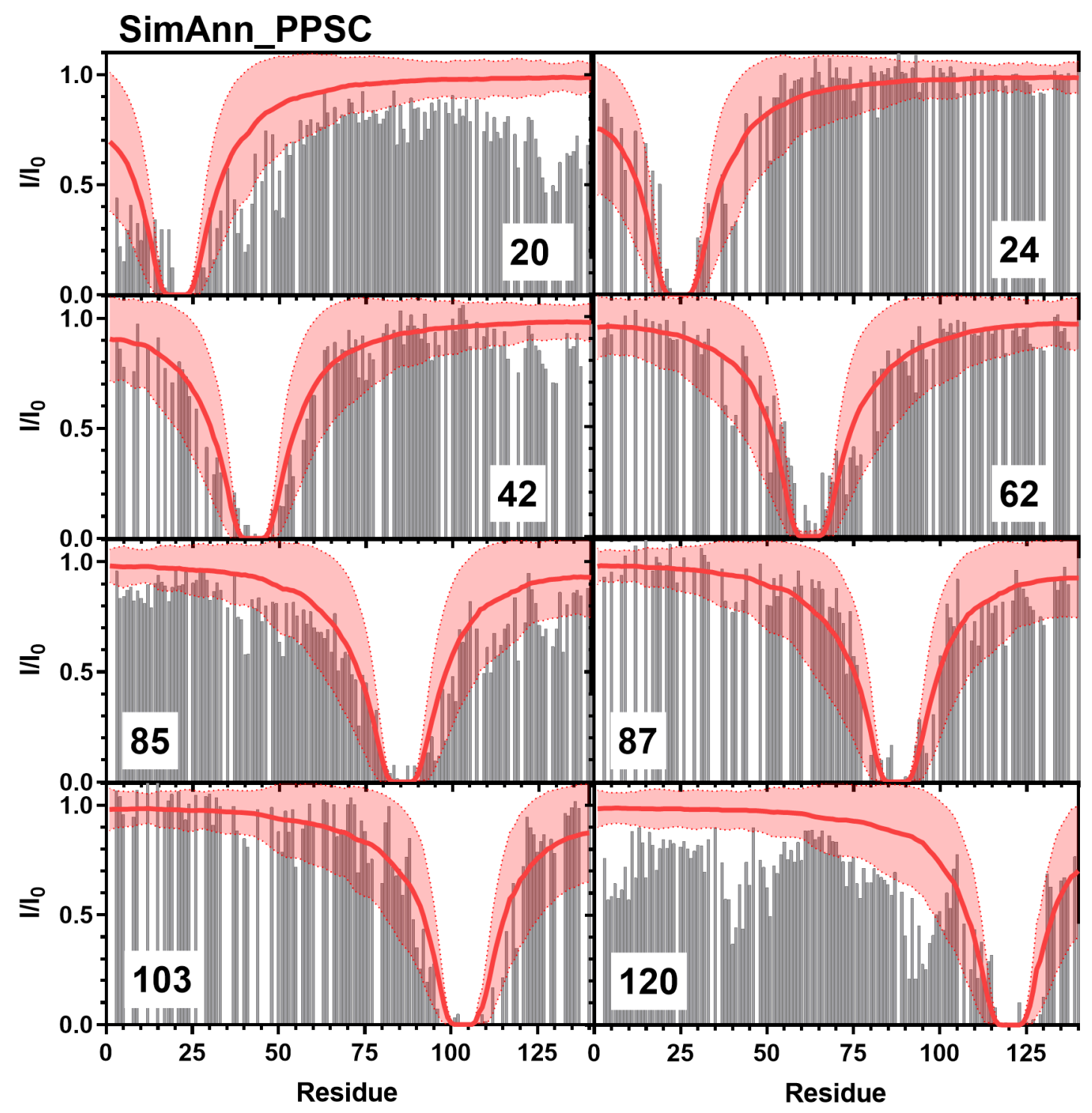

Figure S21: Comparison of Simulated PRE Values and Experimental PRE Values: Simulated PRE values from SimAnn_PPSC (red line) overlayed on top of experimental data (grey bars) from positions 20 (Top Left), 24 (Top Right), 42 (Upper Middle Left), 62 (Upper Middle Right), 85 (Lower Middle Left), 87 (Lower Middle Right), 103 (Bottom Left), 120 (Bottom Right). Experimental data for positions 20, 85, and 120 are from Sung et al. ${ }^{8}$ and data for positions 24 , 42, 62, 87, and 103 are from Dedmon et al. ${ }^{9}$. 


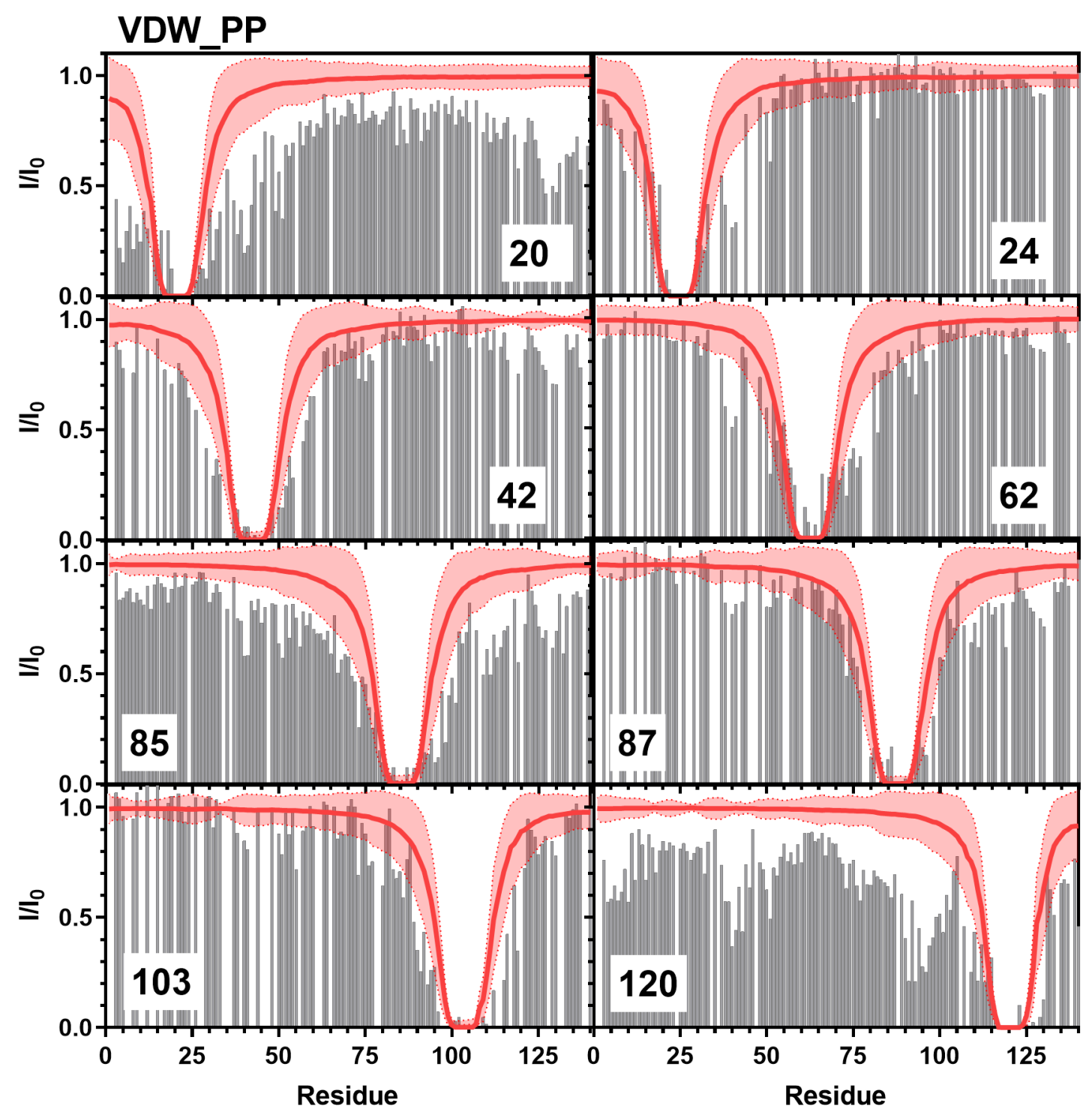

Figure S22: Comparison of Simulated PRE Values and Experimental PRE Values: Simulated PRE values from VDW_PP (red line) overlayed on top of experimental data (grey bars) from positions 20 (Top Left), 24 (Top Right), 42 (Upper Middle Left), 62 (Upper Middle Right), 85 (Lower Middle Left), 87 (Lower Middle Right), 103 (Bottom Left), 120 (Bottom Right). Experimental data for positions 20, 85, and 120 are from Sung et al. ${ }^{8}$ and data for positions $24,42,62,87$, and 103 are from Dedmon et al. ${ }^{9}$. 


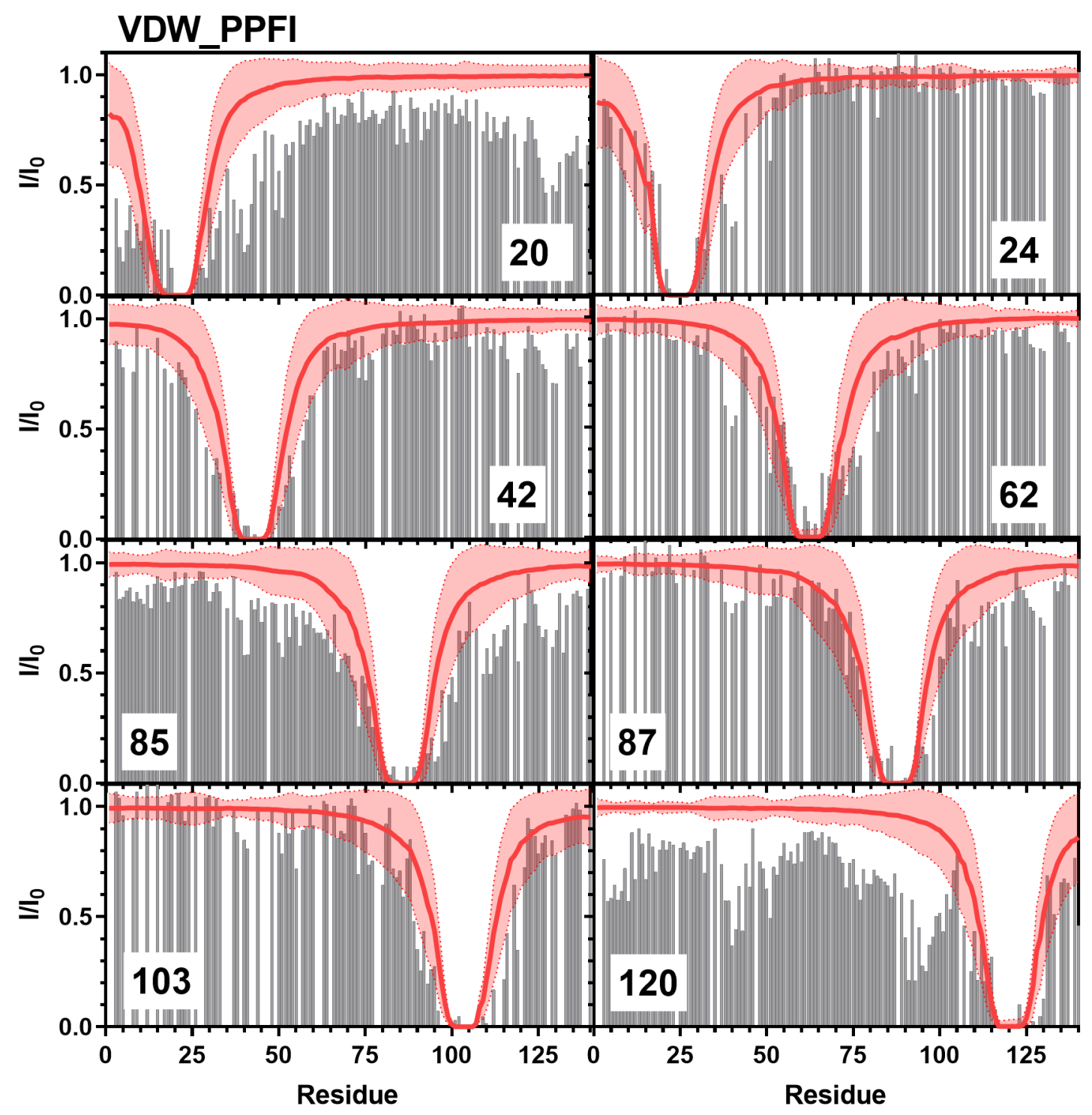

Figure S23: Comparison of Simulated PRE Values and Experimental PRE Values: Simulated PRE values from VDW_PPFI (red line) overlayed on top of experimental data (grey bars) from positions 20 (Top Left), 24 (Top Right), 42 (Upper Middle Left), 62 (Upper Middle Right), 85 (Lower Middle Left), 87 (Lower Middle Right), 103 (Bottom Left), 120 (Bottom Right). Experimental data for positions 20, 85, and 120 are from Sung et al. ${ }^{8}$ and data for positions 24 , 42, 62, 87, and 103 are from Dedmon et al. ${ }^{9}$. 


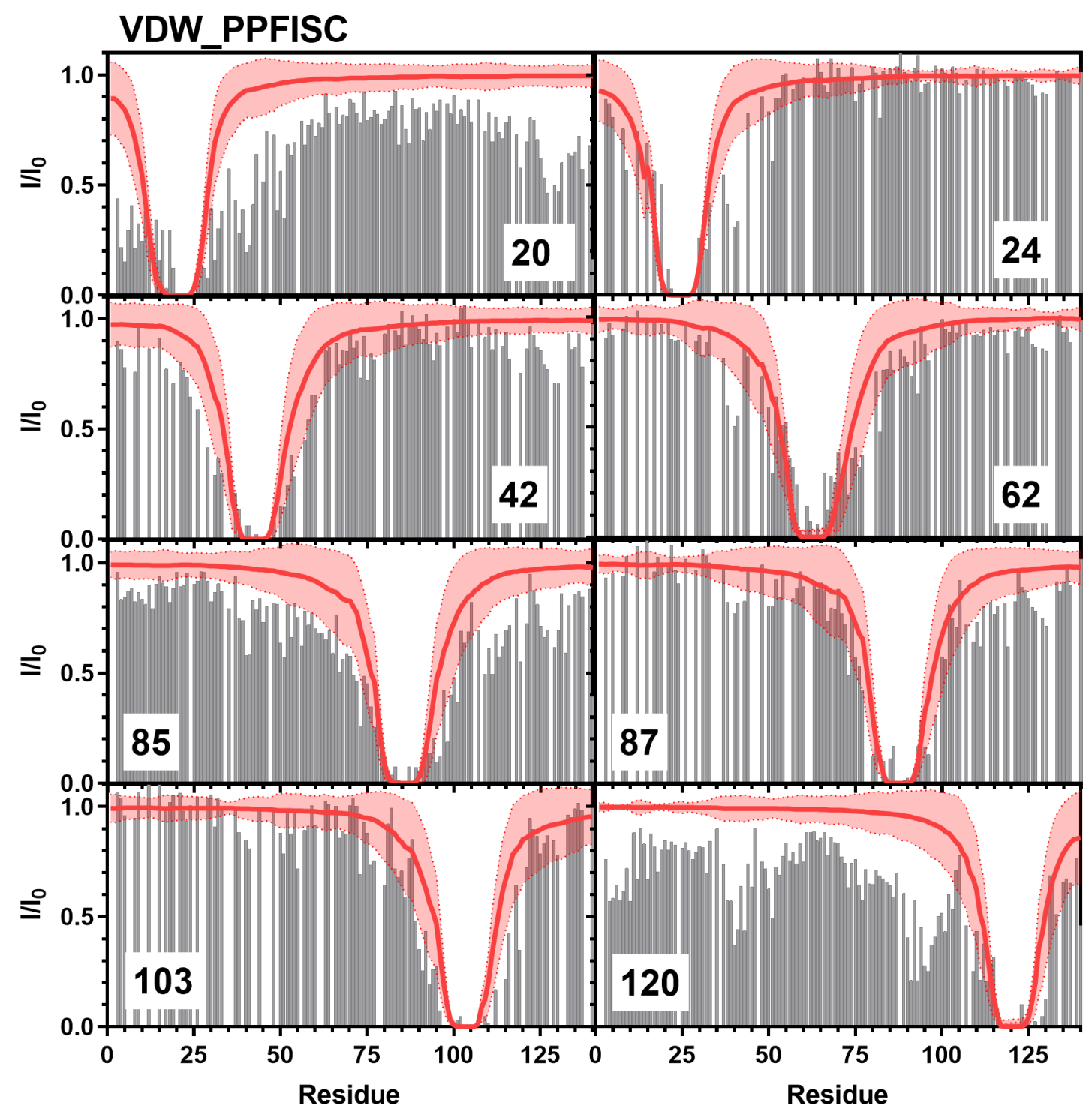

Figure S24: Comparison of Simulated PRE Values and Experimental PRE Values: Simulated PRE values from VDW_PPFISC (red line) overlayed on top of experimental data (grey bars) from positions 20 (Top Left), 24 (Top Right), 42 (Upper Middle Left), 62 (Upper Middle Right), 85 (Lower Middle Left), 87 (Lower Middle Right), 103 (Bottom Left), 120 (Bottom Right). Experimental data for positions 20, 85, and 120 are from Sung et al. ${ }^{8}$ and data for positions 24 , 42, 62, 87, and 103 are from Dedmon et al. ${ }^{9}$. 


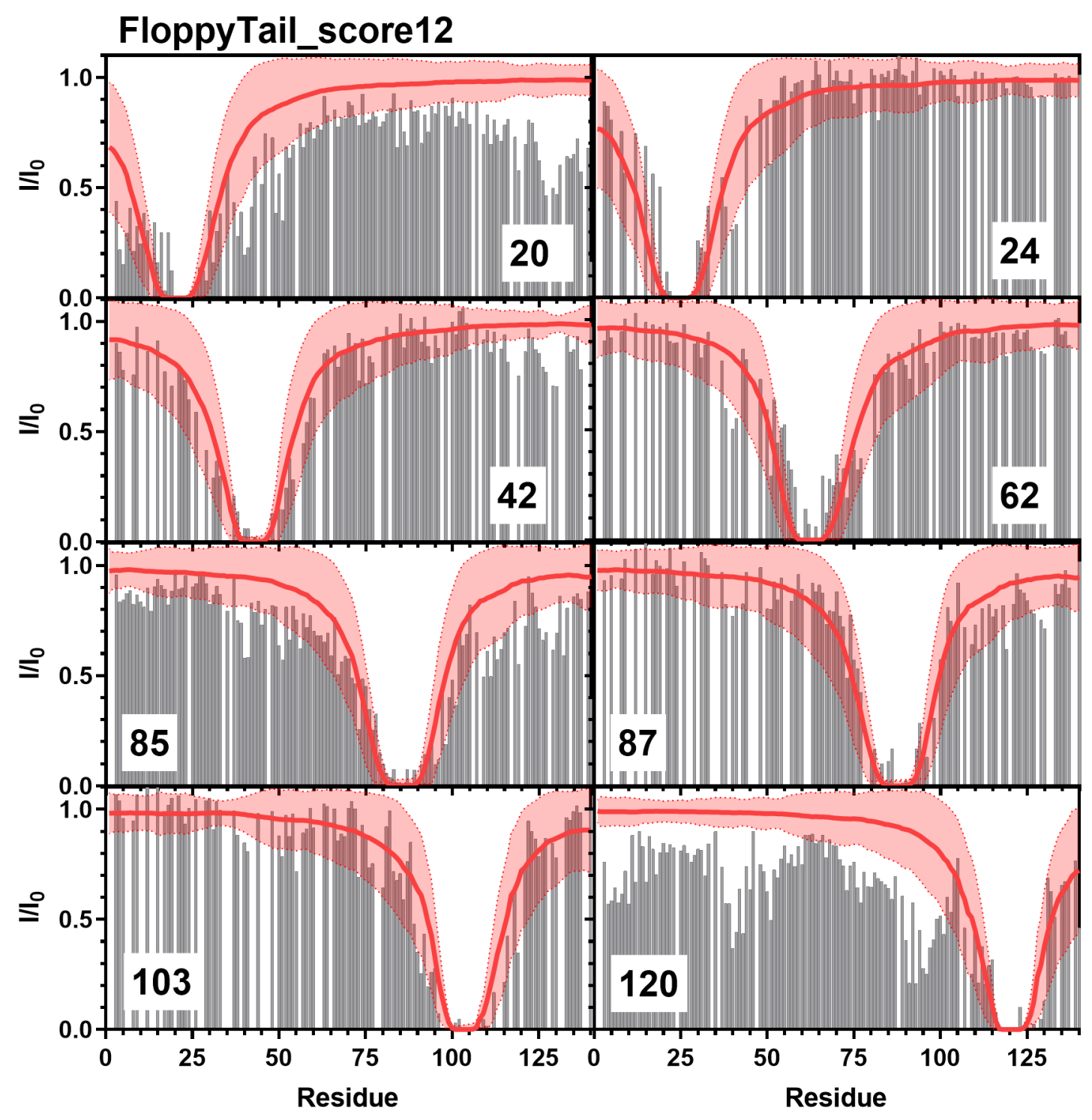

Figure S25: Comparison of Simulated PRE Values and Experimental PRE Values: Simulated PRE values from FloppyTail_score12 (red line) overlayed on top of experimental data (grey bars) from positions 20 (Top Left), 24 (Top Right), 42 (Upper Middle Left), 62 (Upper Middle Right), 85 (Lower Middle Left), 87 (Lower Middle Right), 103 (Bottom Left), 120 (Bottom Right). Experimental data for positions 20, 85, and 120 are from Sung et al. ${ }^{8}$ and data for positions 24 , 42, 62, 87, and 103 are from Dedmon et al. ${ }^{9}$. 


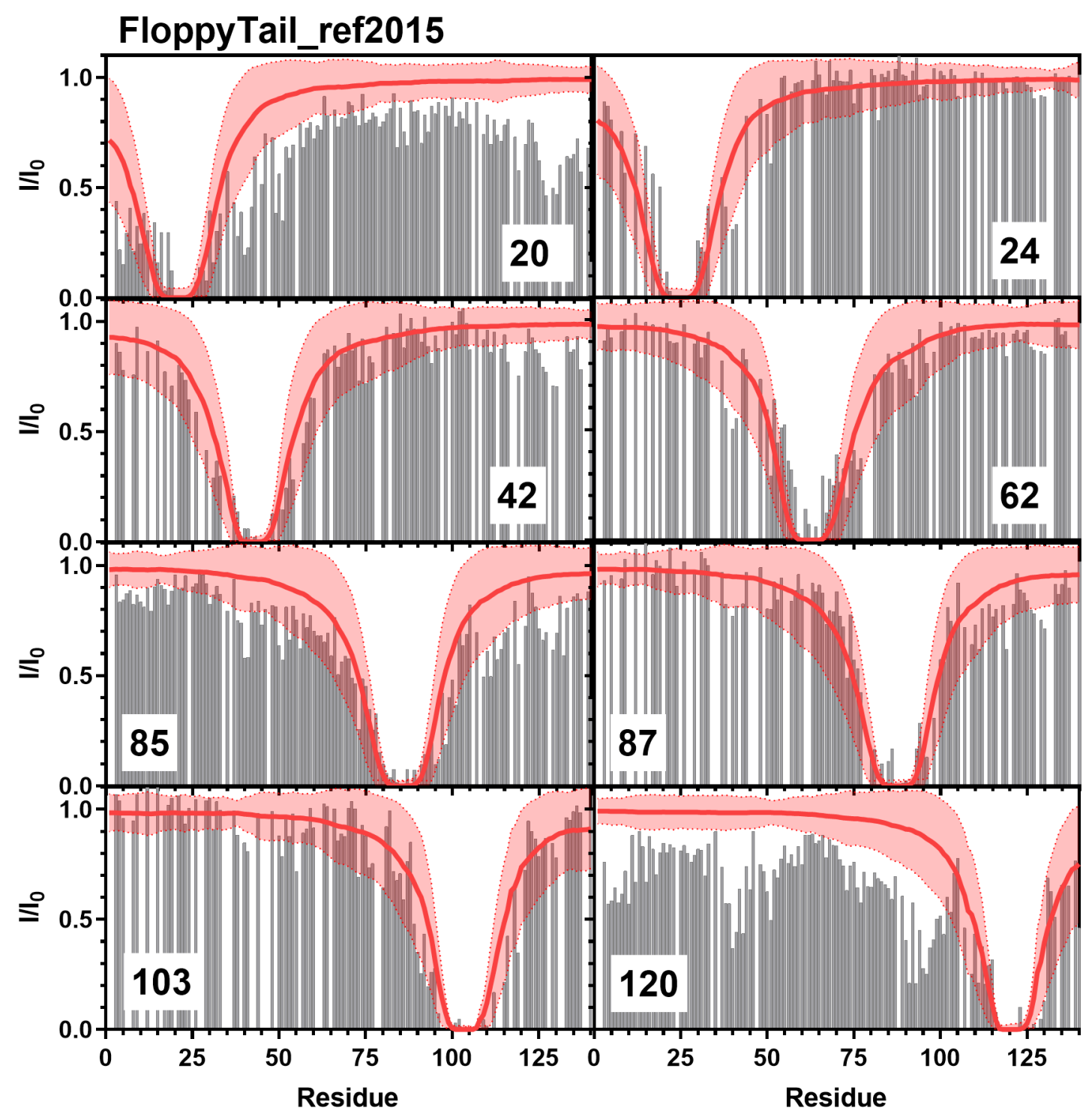

Figure S26: Comparison of Simulated PRE Values and Experimental PRE Values: Simulated PRE values from FloppyTail_ref2015 (red line) overlayed on top of experimental data (grey bars) from positions 20 (Top Left), 24 (Top Right), 42 (Upper Middle Left), 62 (Upper Middle Right), 85 (Lower Middle Left), 87 (Lower Middle Right), 103 (Bottom Left), 120 (Bottom Right). Experimental data for positions 20, 85, and 120 are from Sung et al. ${ }^{8}$ and data for positions 24 , 42, 62, 87, and 103 are from Dedmon et al. ${ }^{9}$. 


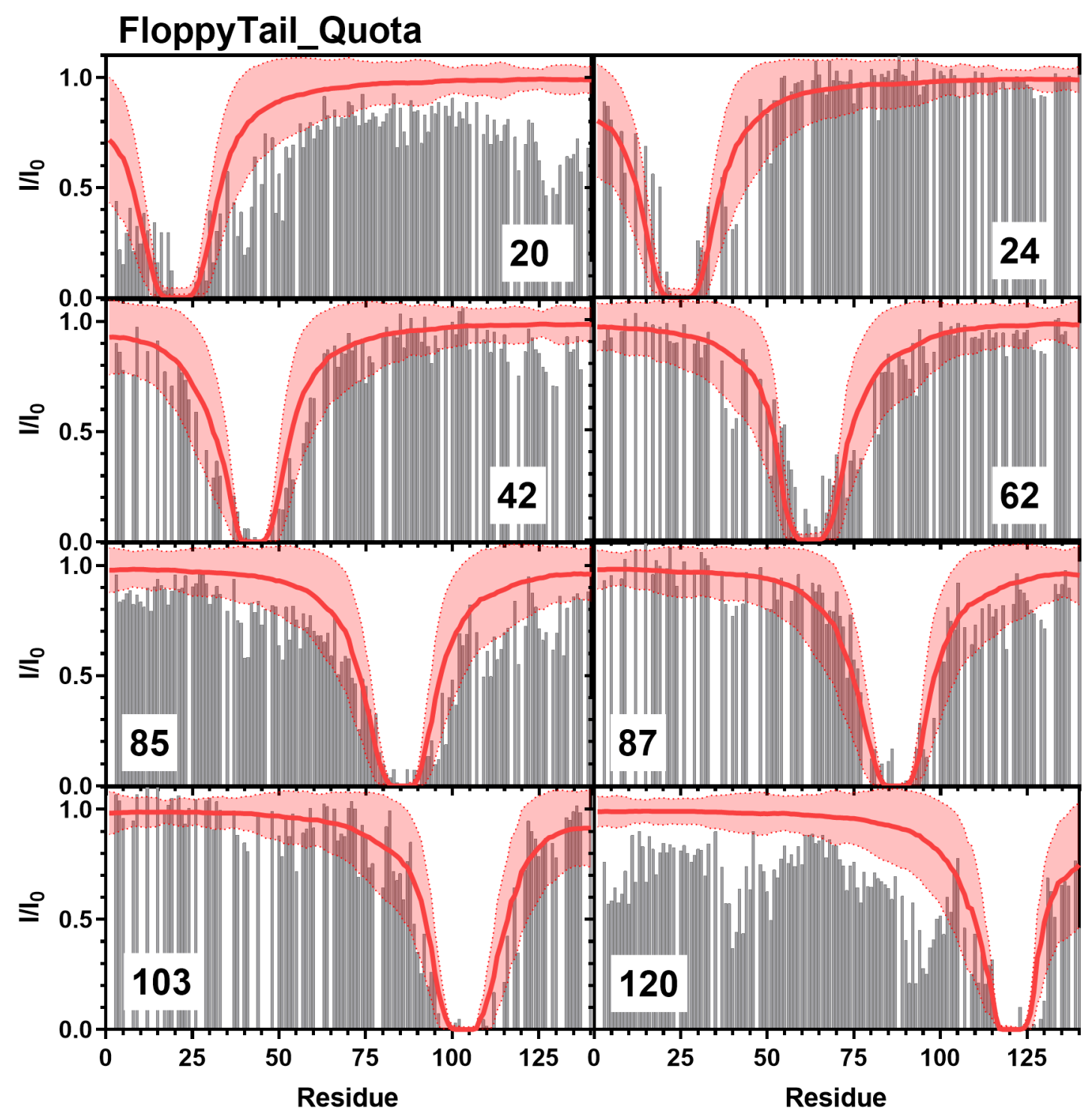

Figure S27: Comparison of Simulated PRE Values and Experimental PRE Values: Simulated PRE values from FloppyTail_Quota (red line) overlayed on top of experimental data (grey bars) from positions 20 (Top Left), 24 (Top Right), 42 (Upper Middle Left), 62 (Upper Middle Right), 85 (Lower Middle Left), 87 (Lower Middle Right), 103 (Bottom Left), 120 (Bottom Right). Experimental data for positions 20, 85, and 120 are from Sung et al. ${ }^{8}$ and data for positions 24 , 42, 62, 87, and 103 are from Dedmon et al. ${ }^{9}$. 


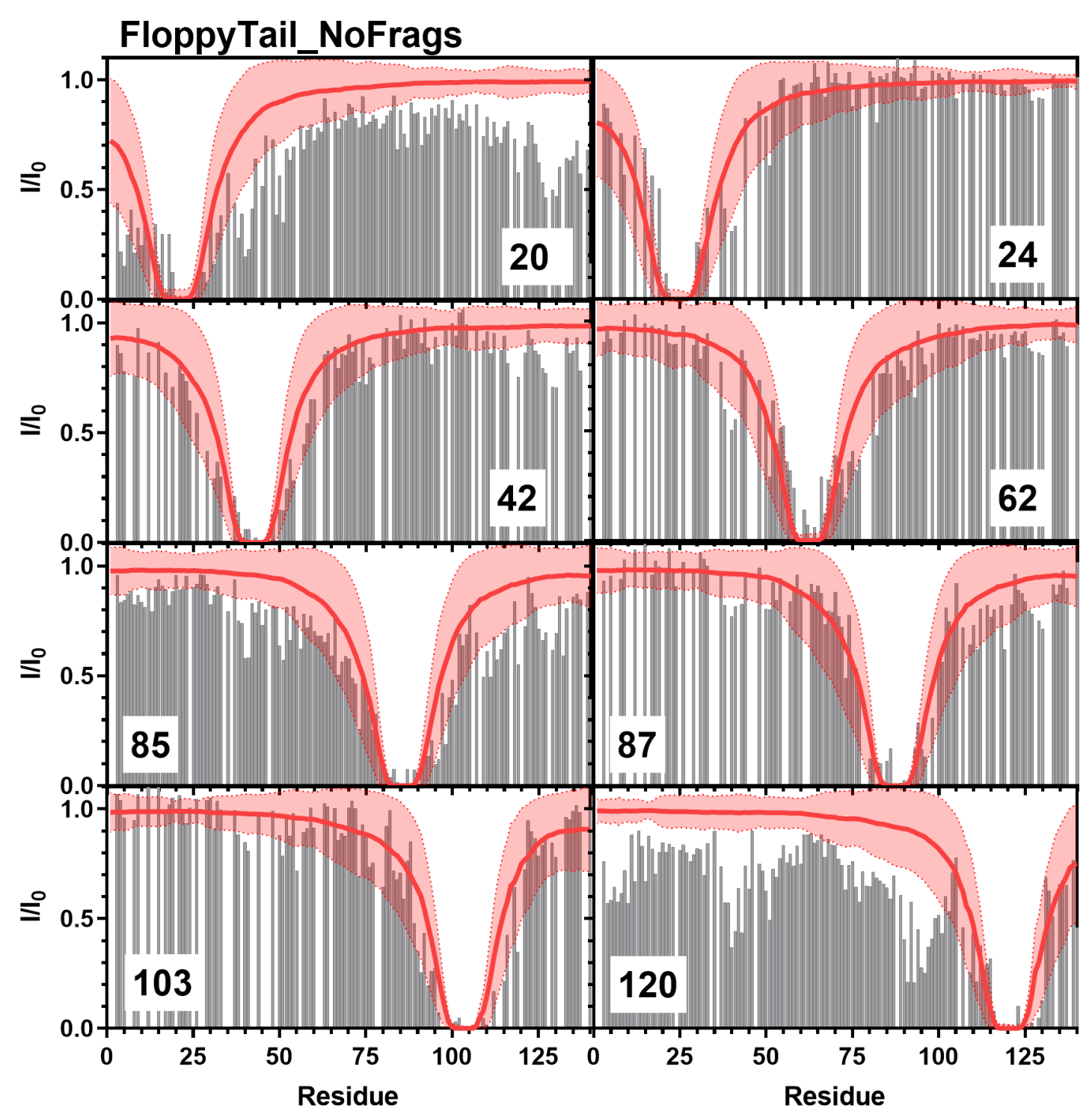

Figure S28: Comparison of Simulated PRE Values and Experimental PRE Values: Simulated PRE values from FloppyTail_NoFrags (red line) overlayed on top of experimental data (grey bars) from positions 20 (Top Left), 24 (Top Right), 42 (Upper Middle Left), 62 (Upper Middle Right), 85 (Lower Middle Left), 87 (Lower Middle Right), 103 (Bottom Left), 120 (Bottom Right). Experimental data for positions 20, 85, and 120 are from Sung et al. ${ }^{8}$ and data for positions 24 , 42, 62, 87, and 103 are from Dedmon et al. ${ }^{9}$. 


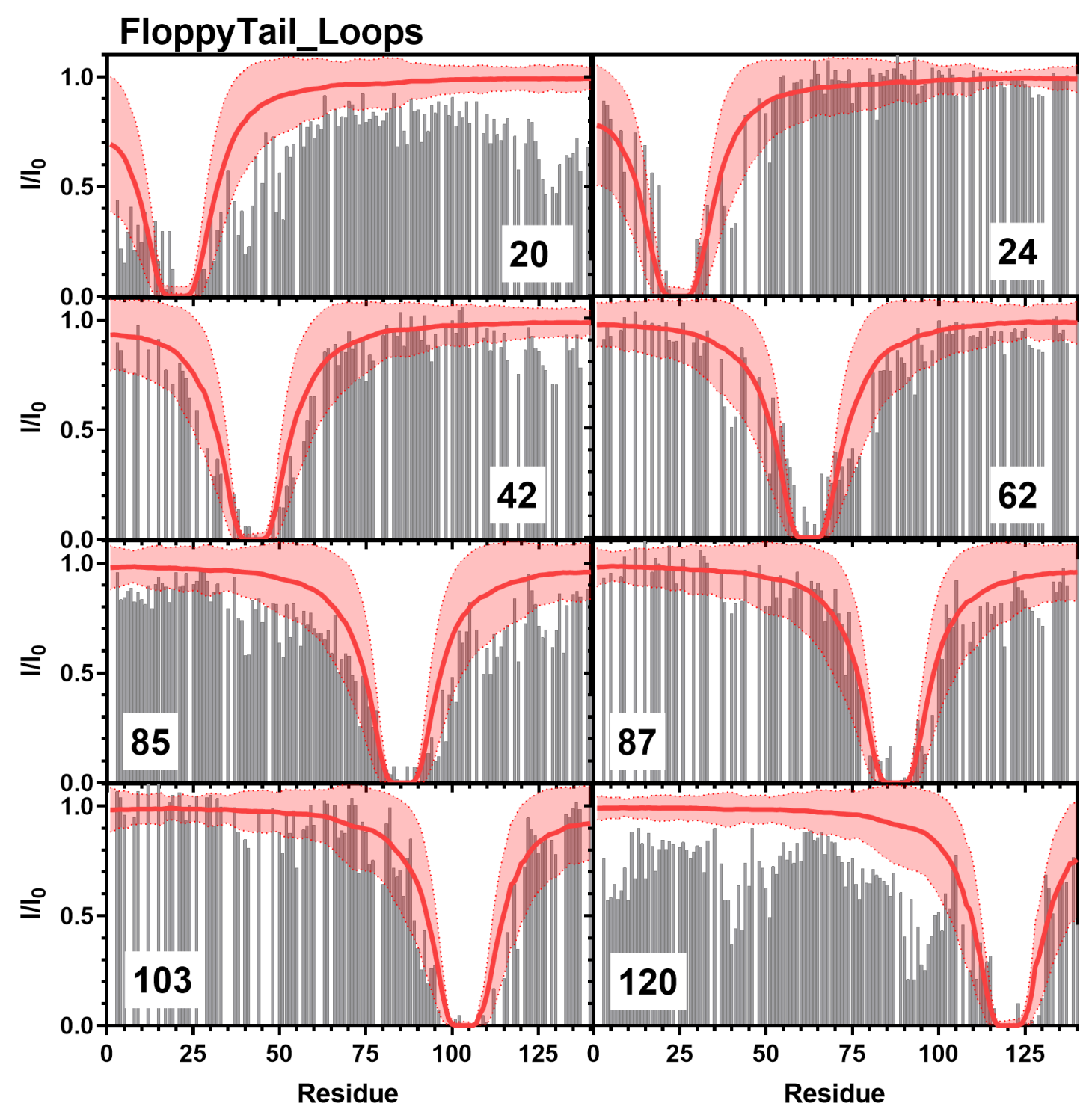

Figure S29: Comparison of Simulated PRE Values and Experimental PRE Values: Simulated PRE values from FloppyTail_Loops (red line) overlayed on top of experimental data (grey bars) from positions 20 (Top Left), 24 (Top Right), 42 (Upper Middle Left), 62 (Upper Middle Right), 85 (Lower Middle Left), 87 (Lower Middle Right), 103 (Bottom Left), 120 (Bottom Right). Experimental data for positions 20, 85, and 120 are from Sung et al. ${ }^{8}$ and data for positions 24, 42, 62, 87, and 103 are from Dedmon et al. ${ }^{9}$, 


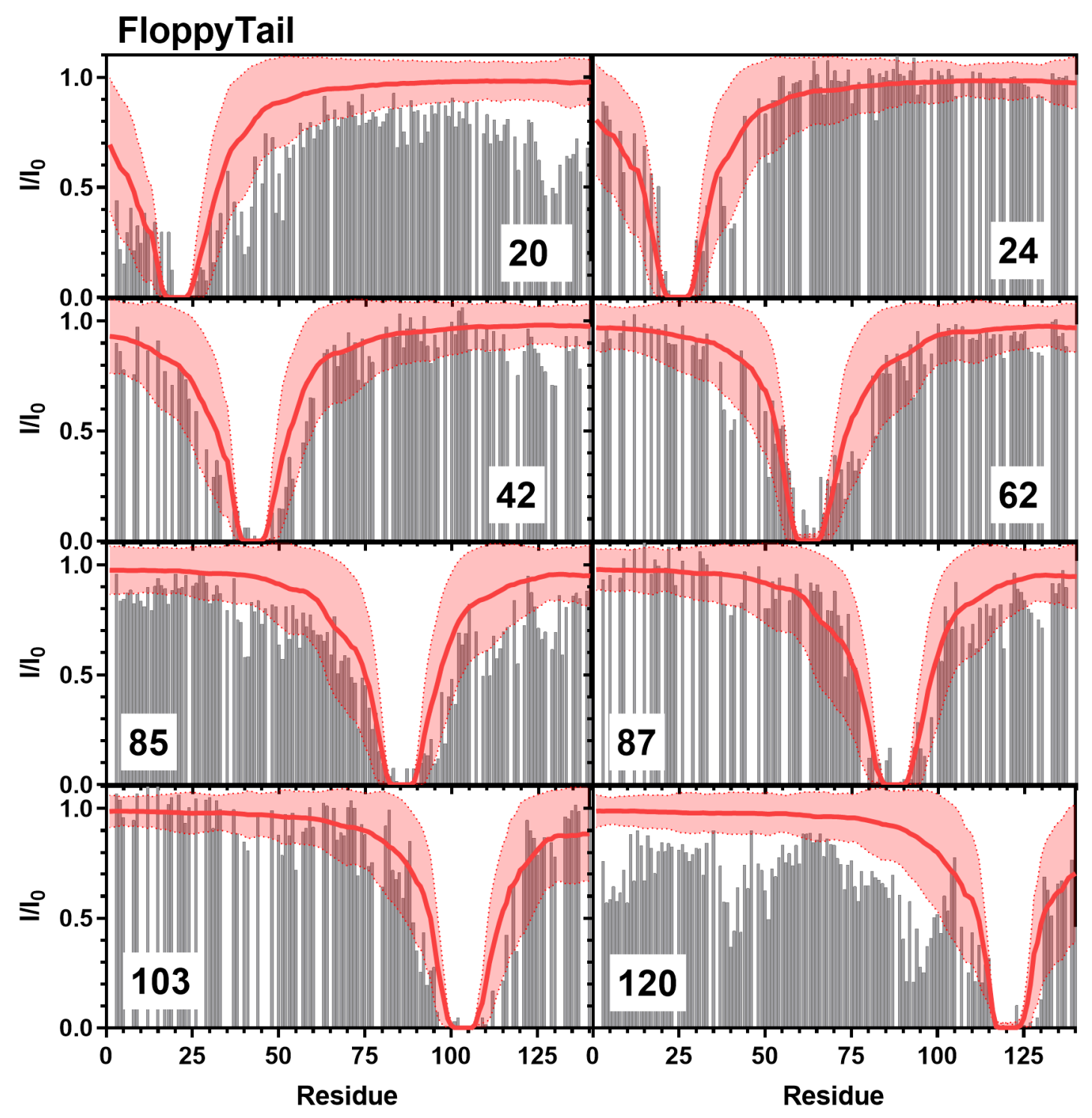

Figure S30: Comparison of Simulated PRE Values and Experimental PRE Values: Simulated PRE values from FloppyTail (red line) overlayed on top of experimental data (grey bars) from positions 20 (Top Left), 24 (Top Right), 42 (Upper Middle Left), 62 (Upper Middle Right), 85 (Lower Middle Left), 87 (Lower Middle Right), 103 (Bottom Left), 120 (Bottom Right). Experimental data for positions 20, 85, and 120 are from Sung et al. ${ }^{8}$ and data for positions $24,42,62,87$, and 103 are from Dedmon et al. ${ }^{9}$. 


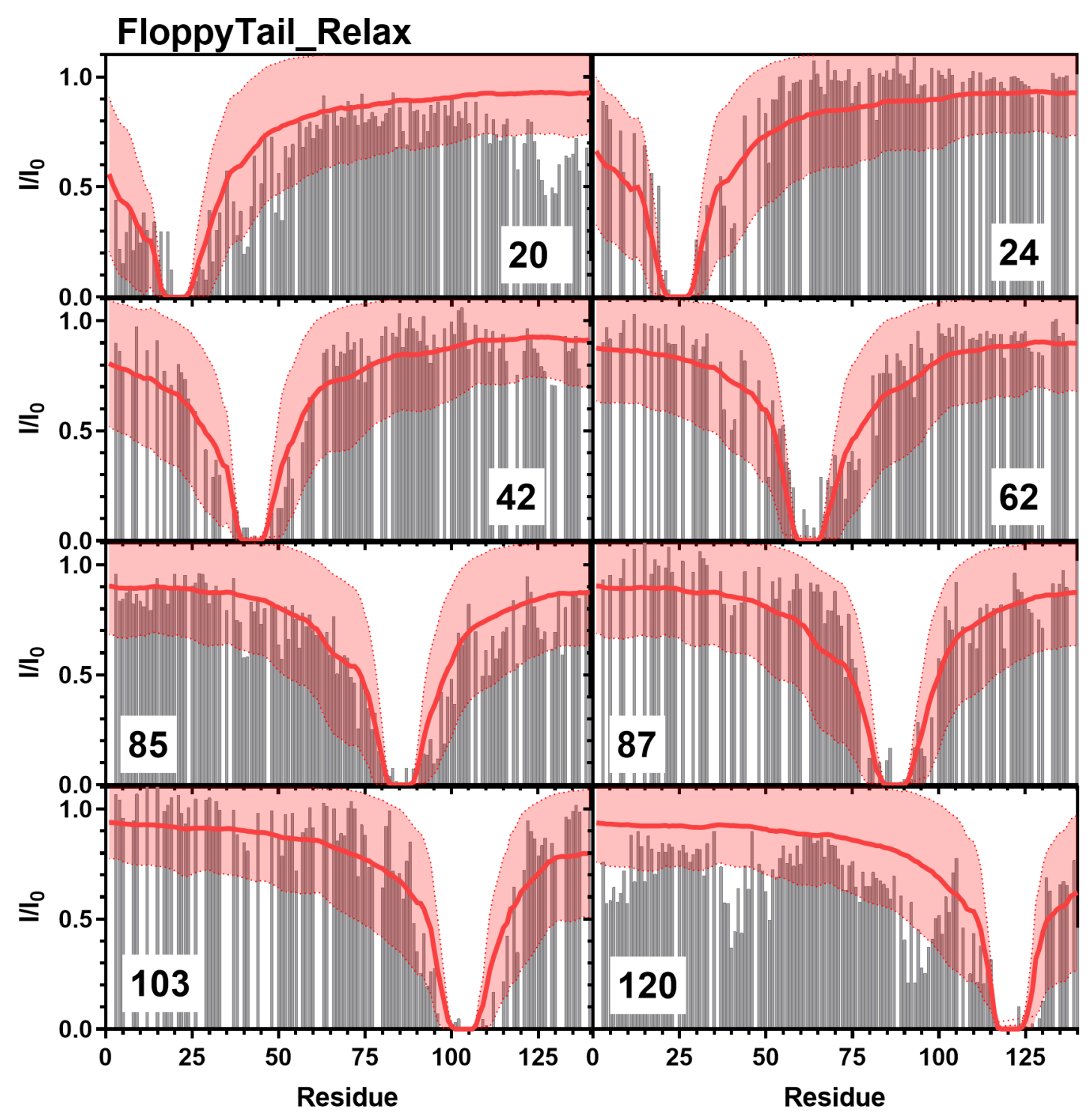

Figure S31: Comparison of Simulated PRE Values and Experimental PRE Values: Simulated PRE values from FloppyTail_Relax (red line) overlayed on top of experimental data (grey bars) from positions 20 (Top Left), 24 (Top Right), 42 (Upper Middle Left), 62 (Upper Middle Right), 85 (Lower Middle Left), 87 (Lower Middle Right), 103 (Bottom Left), 120 (Bottom Right). Experimental data for positions 20, 85, and 120 are from Sung et al. ${ }^{8}$ and data for positions 24 , 42, 62, 87, and 103 are from Dedmon et al. ${ }^{9}$. 


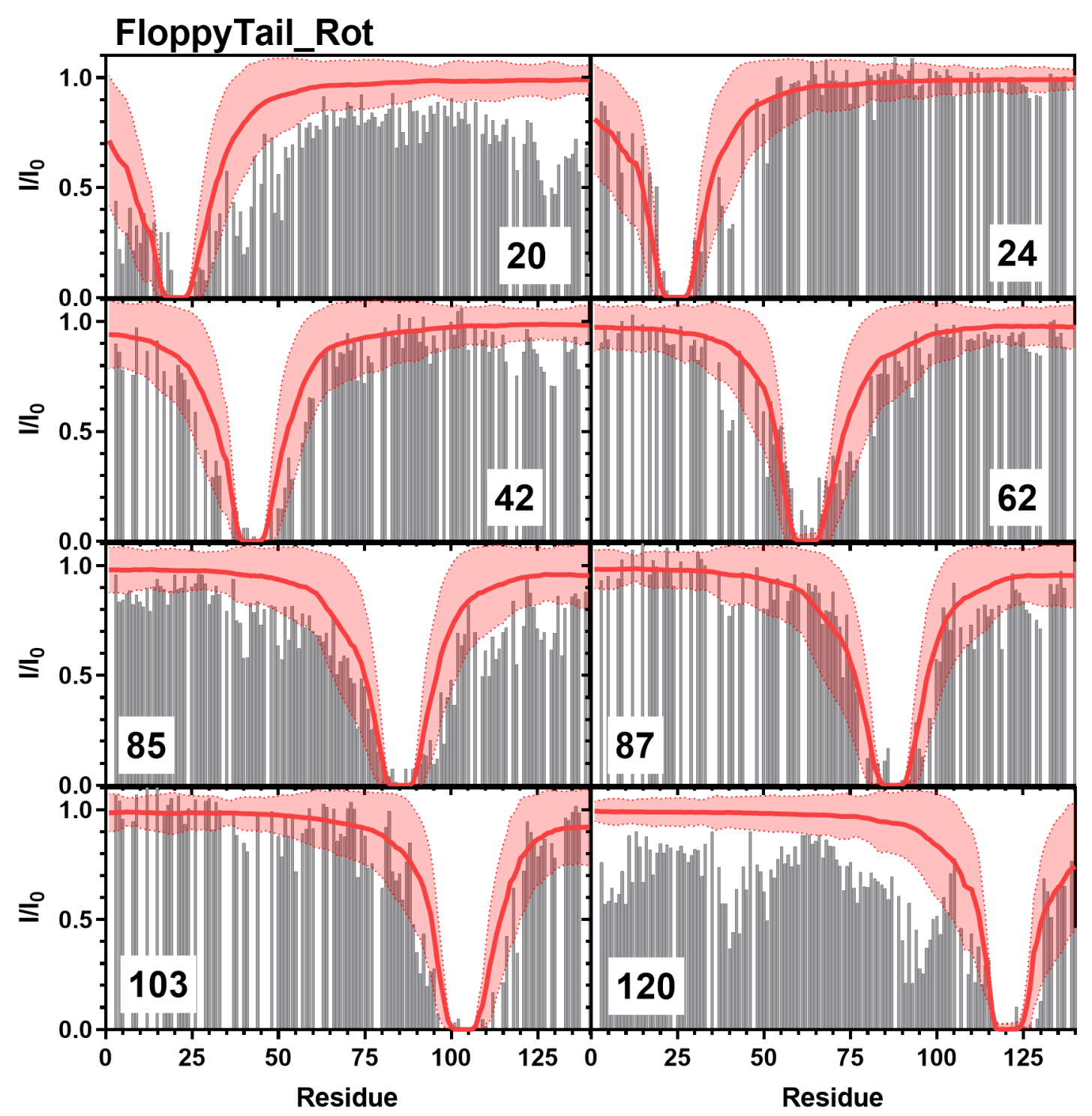

Figure S32: Comparison of Simulated PRE Values and Experimental PRE Values: Simulated PRE values from FloppyTail_Rot (red line) overlayed on top of experimental data (grey bars) from positions 20 (Top Left), 24 (Top Right), 42 (Upper Middle Left), 62 (Upper Middle Right), 85 (Lower Middle Left), 87 (Lower Middle Right), 103 (Bottom Left), 120 (Bottom Right). Experimental data for positions 20, 85, and 120 are from Sung et al. ${ }^{8}$ and data for positions 24 , 42, 62, 87, and 103 are from Dedmon et al. ${ }^{9}$. 


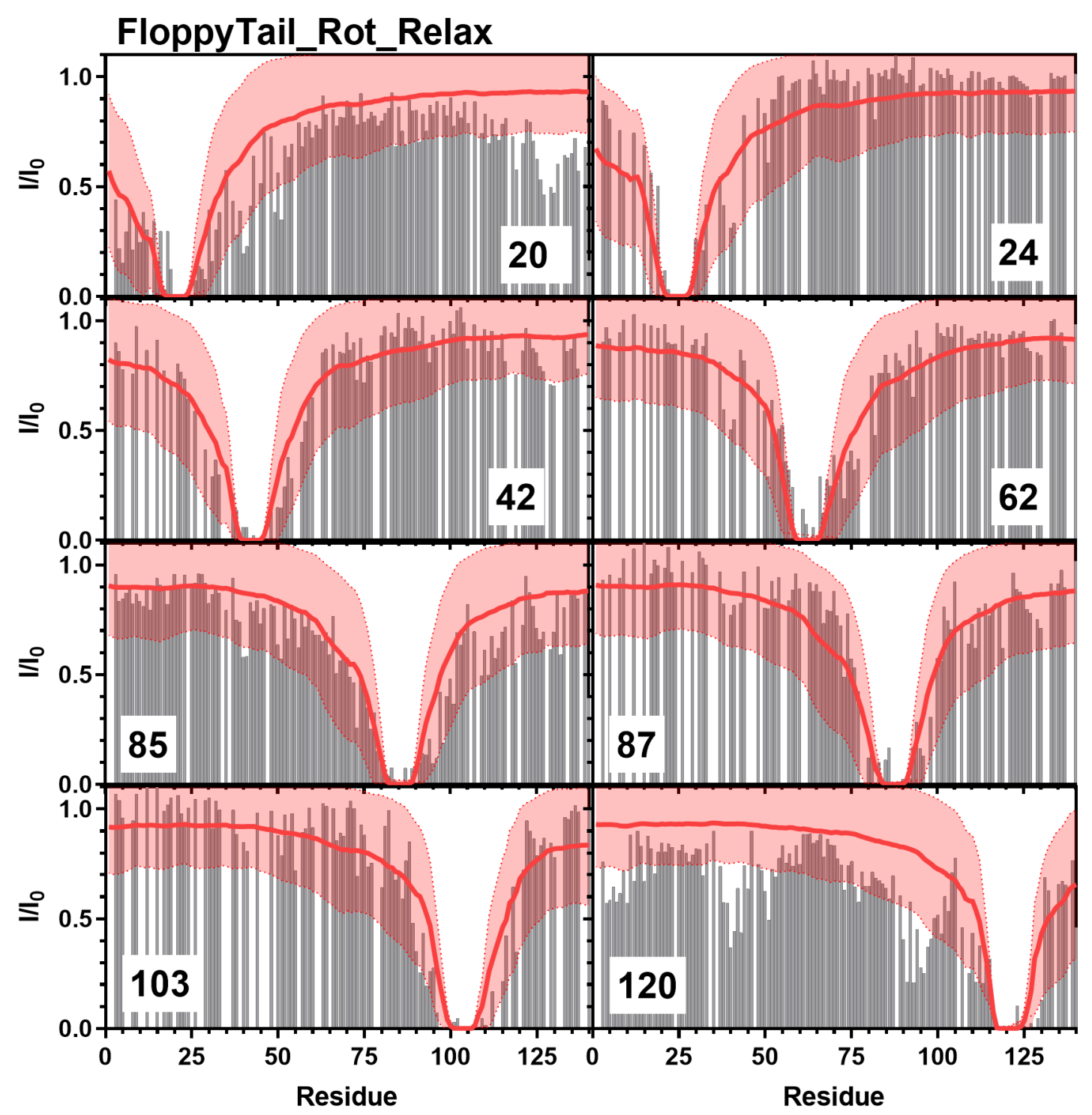

Figure S33: Comparison of Simulated PRE Values and Experimental PRE Values: Simulated PRE values from FloppyTail_Rot_Relax (red line) overlayed on top of experimental data (grey bars) from positions 20 (Top Left), 24 (Top Right), 42 (Upper Middle Left), 62 (Upper Middle Right), 85 (Lower Middle Left), 87 (Lower Middle Right), 103 (Bottom Left), 120 (Bottom Right). Experimental data for positions 20, 85, and 120 are from Sung et al. ${ }^{8}$ and data for positions 24, 42, 62, 87, and 103 are from Dedmon et al. ${ }^{9}$. 


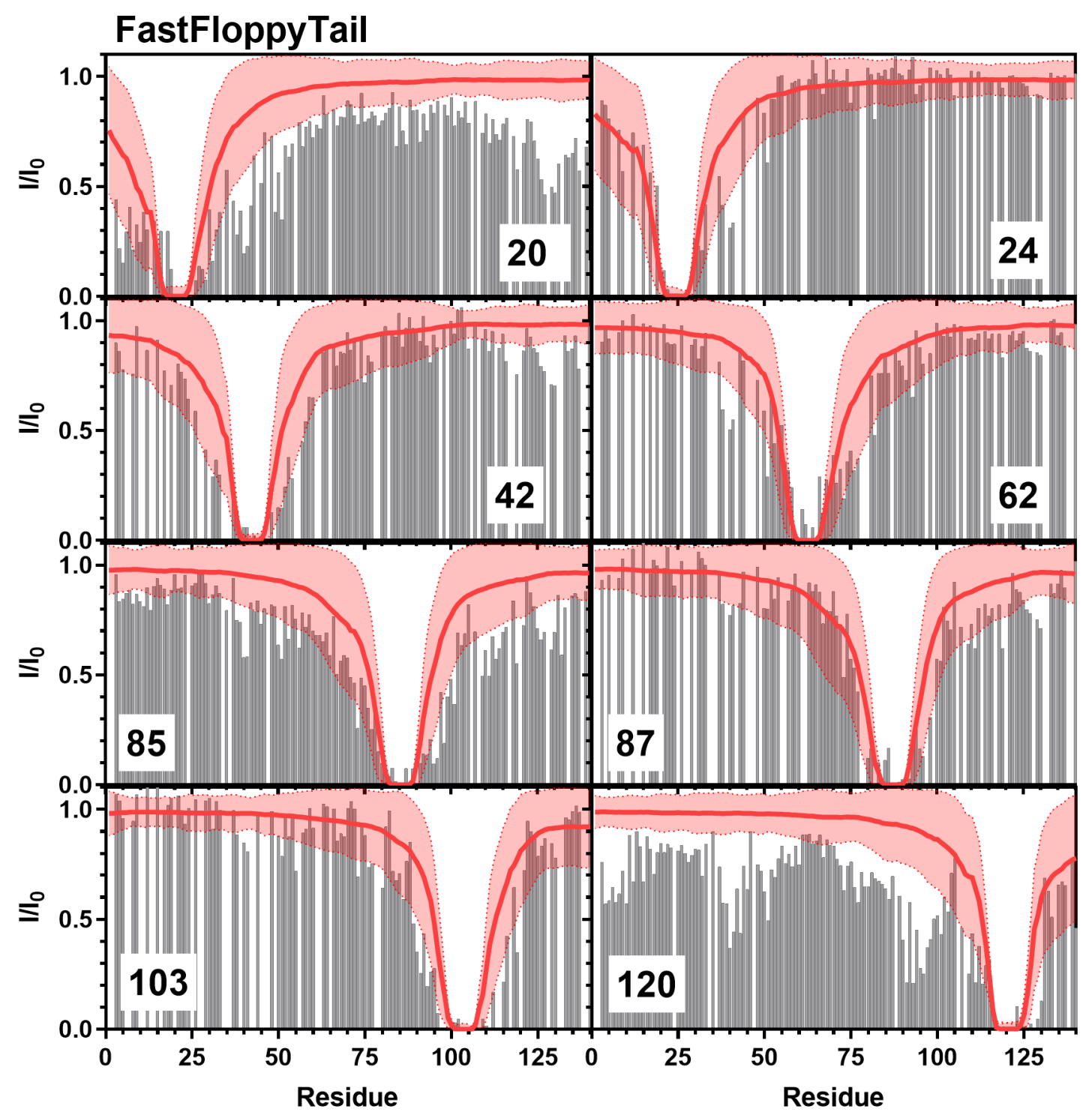

Figure S34: Comparison of Simulated PRE Values and Experimental PRE Values: Simulated PRE values from FastFloppyTail (red line) overlayed on top of experimental data (grey bars) from positions 20 (Top Left), 24 (Top Right), 42 (Upper Middle Left), 62 (Upper Middle Right), 85 (Lower Middle Left), 87 (Lower Middle Right), 103 (Bottom Left), 120 (Bottom Right). Experimental data for positions 20, 85, and 120 are from Sung et al. ${ }^{8}$ and data for positions 24, 42, 62, 87, and 103 are from Dedmon et al. ${ }^{9}$. 


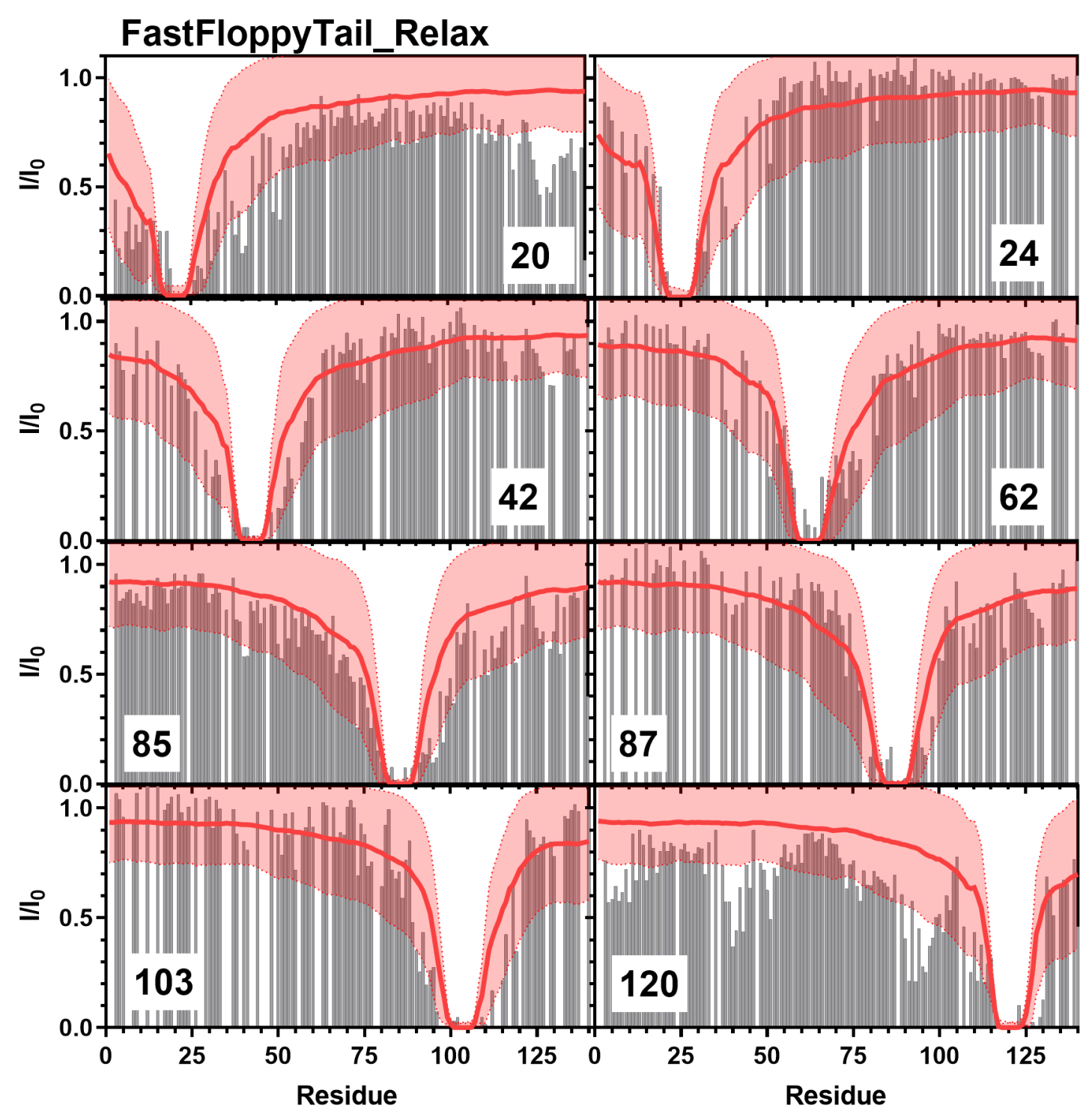

Figure S35: Comparison of Simulated PRE Values and Experimental PRE Values: Simulated PRE values from FastFloppyTail (red line) overlayed on top of experimental data (grey bars) from positions 20 (Top Left), 24 (Top Right), 42 (Upper Middle Left), 62 (Upper Middle Right), 85 (Lower Middle Left), 87 (Lower Middle Right), 103 (Bottom Left), 120 (Bottom Right). Experimental data for positions 20, 85, and 120 are from Sung et al. ${ }^{8}$ and data for positions 24 , 42, 62, 87, and 103 are from Dedmon et al. ${ }^{9}$. 


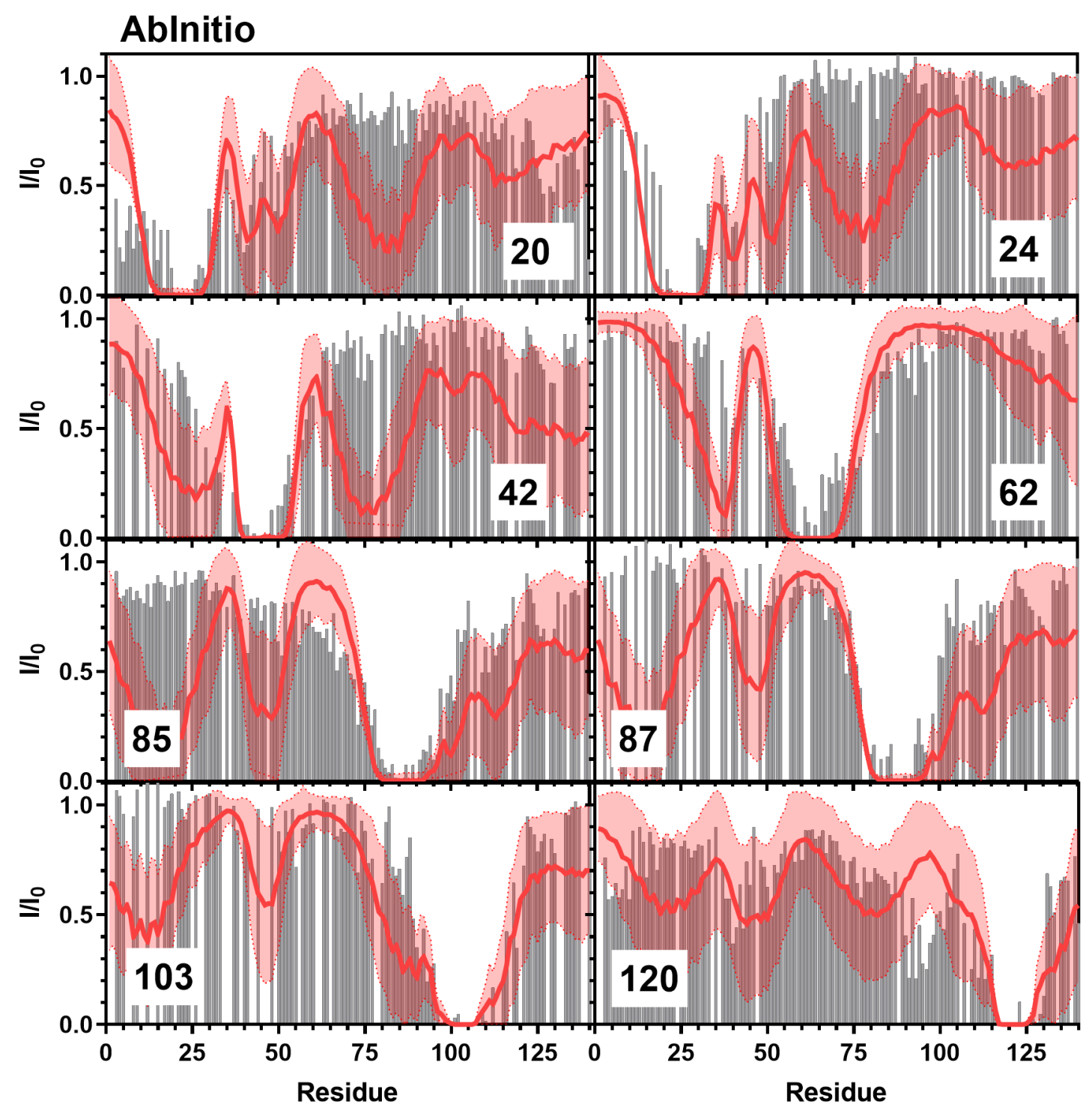

Figure S36: Comparison of Simulated PRE Values and Experimental PRE Values: Simulated PRE values from AbInitio (red line) overlayed on top of experimental data (grey bars) from positions 20 (Top Left), 24 (Top Right), 42 (Upper Middle Left), 62 (Upper Middle Right), 85 (Lower Middle Left), 87 (Lower Middle Right), 103 (Bottom Left), 120 (Bottom Right). Experimental data for positions 20, 85, and 120 are from Sung et al. ${ }^{8}$ and data for positions 24, 42, 62, 87, and 103 are from Dedmon et al. ${ }^{9}$. 


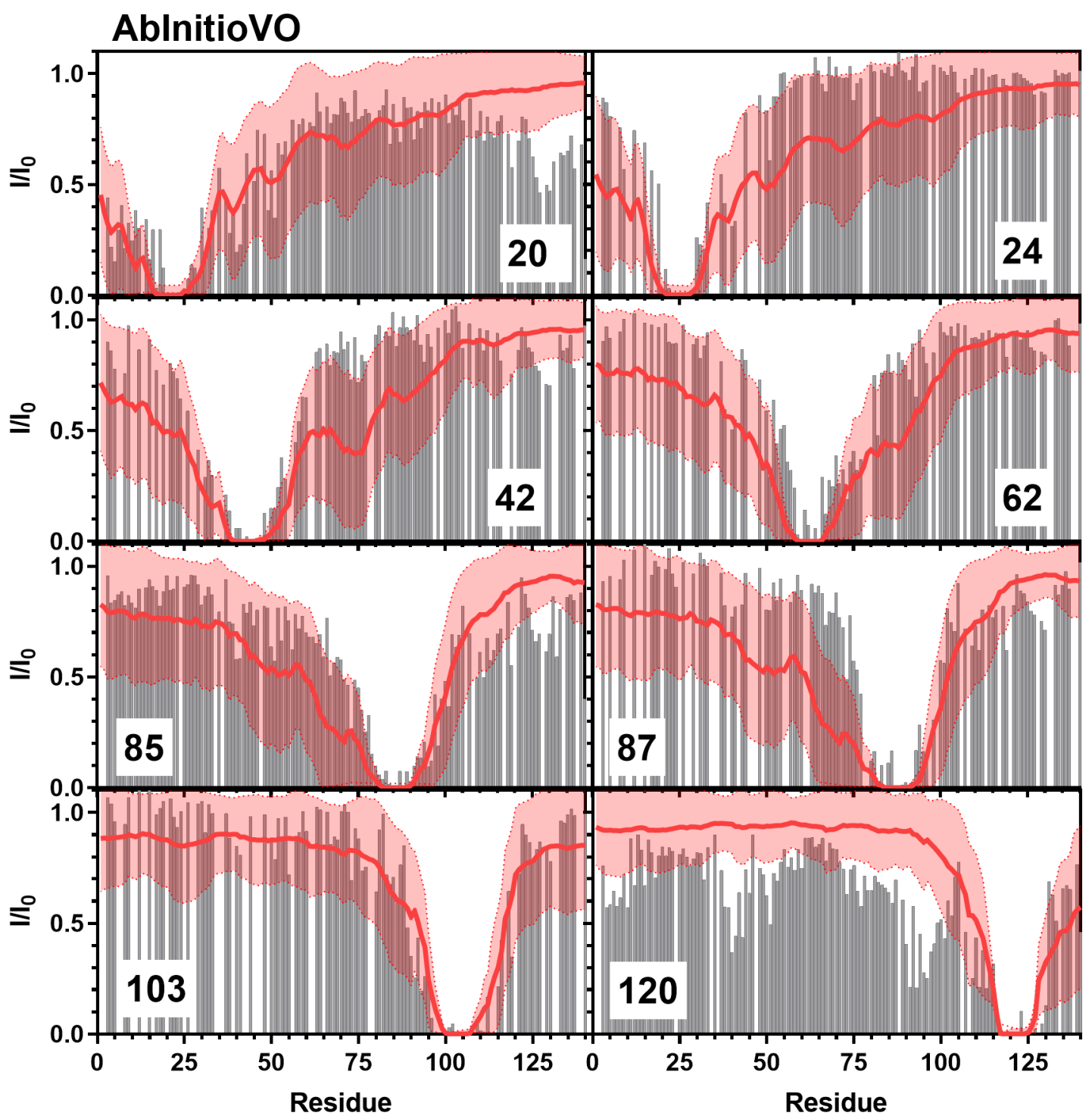

Figure S37: Comparison of Simulated PRE Values and Experimental PRE Values: Simulated PRE values from AbInitioVO (red line) overlayed on top of experimental data (grey bars) from positions 20 (Top Left), 24 (Top Right), 42 (Upper Middle Left), 62 (Upper Middle Right), 85 (Lower Middle Left), 87 (Lower Middle Right), 103 (Bottom Left), 120 (Bottom Right). Experimental data for positions 20, 85, and 120 are from Sung et al. ${ }^{8}$ and data for positions 24 , 42, 62, 87, and 103 are from Dedmon et al. ${ }^{9}$. 


\section{Comparison with $\alpha$ S Chemical Shift Data}

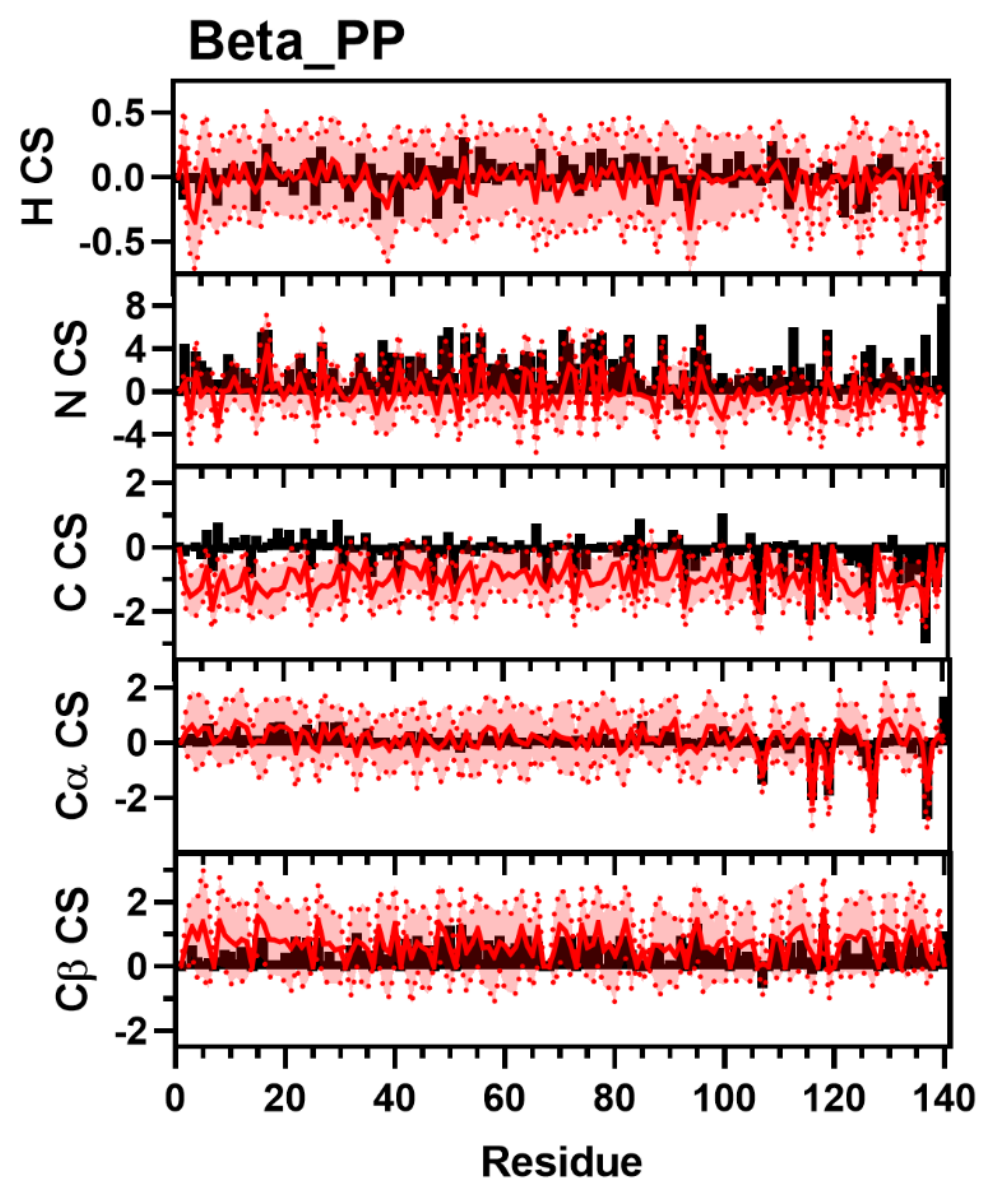

Figure S38: Comparison of Simulated and Experimental NMR Chemical Shift Data. Simulated Chemical Shift values from the Beta_PP ensemble (red) overlayed on experimental data (black bars) of N (Top), H (Upper Middle), $\mathrm{C}$ (Middle), C $\alpha$ (Lower Middle), and C $\beta$ (Bottom) chemical shifts from Sung et al. ${ }^{8}$ Neighbor corrected random coil chemical shift values have been subtracted from both simulated and experimental data ${ }^{11}$. 


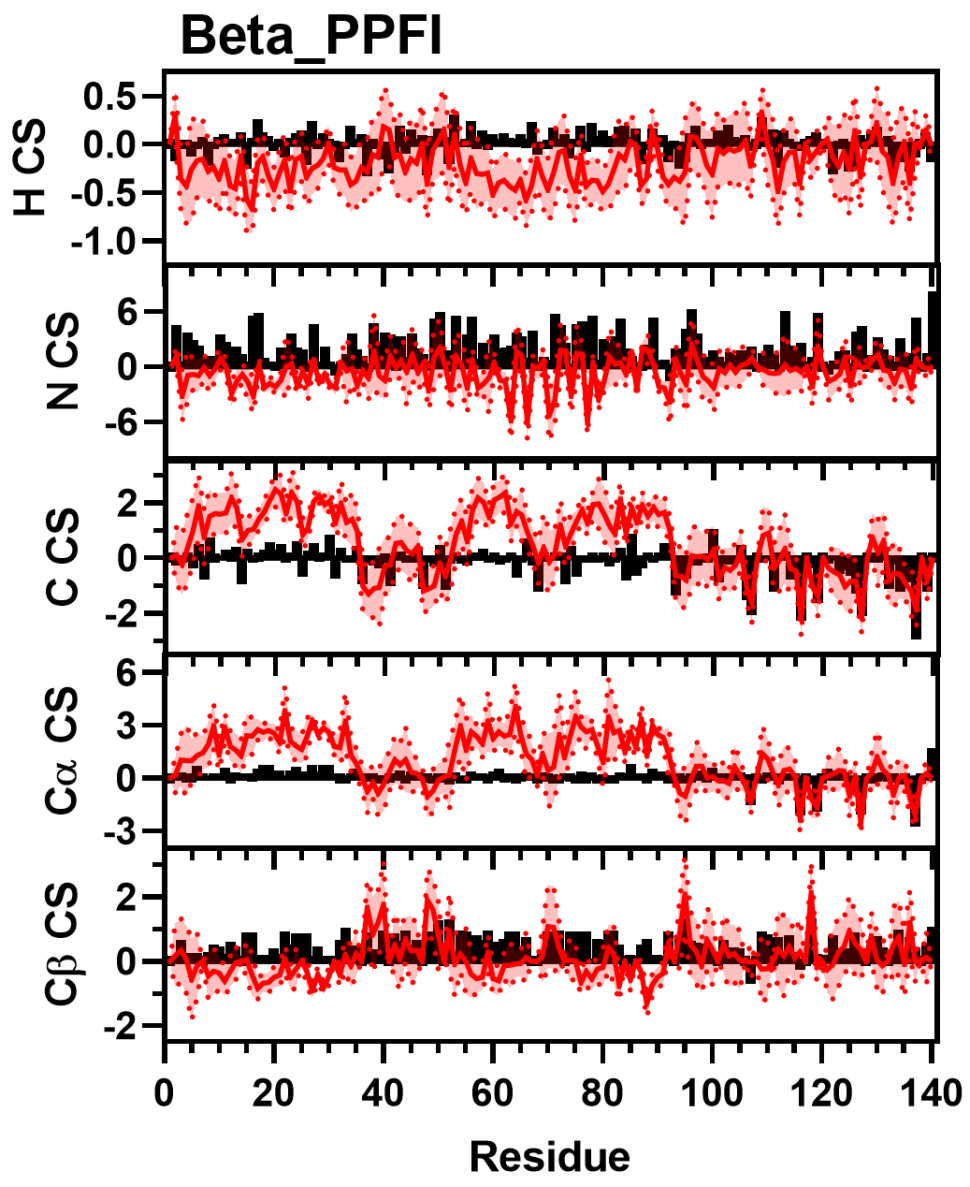

Figure S39: Comparison of Simulated and Experimental NMR Chemical Shift Data. Simulated Chemical Shift values from the Beta_PPFI ensemble (red) overlayed on experimental data (black bars) of N (Top), H (Upper Middle), C (Middle), C $\alpha$ (Lower Middle), and C $\beta$ (Bottom) chemical shifts from Sung et al. ${ }^{8}$ Neighbor corrected random coil chemical shift values have been subtracted from both simulated and experimental data ${ }^{11}$. 


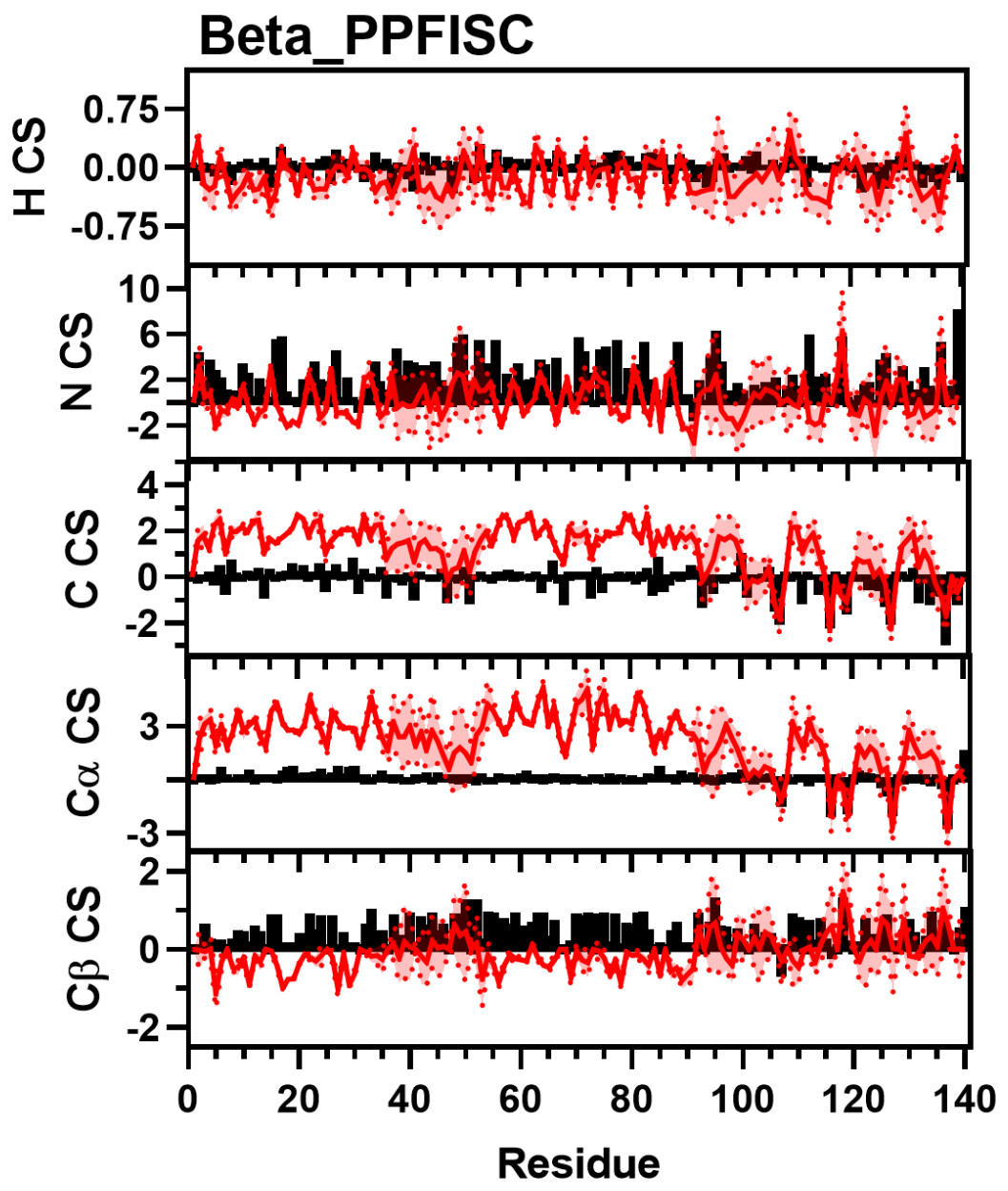

Figure S40: Comparison of Simulated and Experimental NMR Chemical Shift Data. Simulated Chemical Shift values from the Beta_PPFISC ensemble (red) overlayed on experimental data (black bars) of $\mathrm{N}$ (Top), $\mathrm{H}$ (Upper Middle), $\mathrm{C}$ (Middle), $\mathrm{C} \alpha$ (Lower Middle), and C $\beta$ (Bottom) chemical shifts from Sung et al. ${ }^{8}$ Neighbor corrected random coil chemical shift values have been subtracted from both simulated and experimental data ${ }^{11}$. 


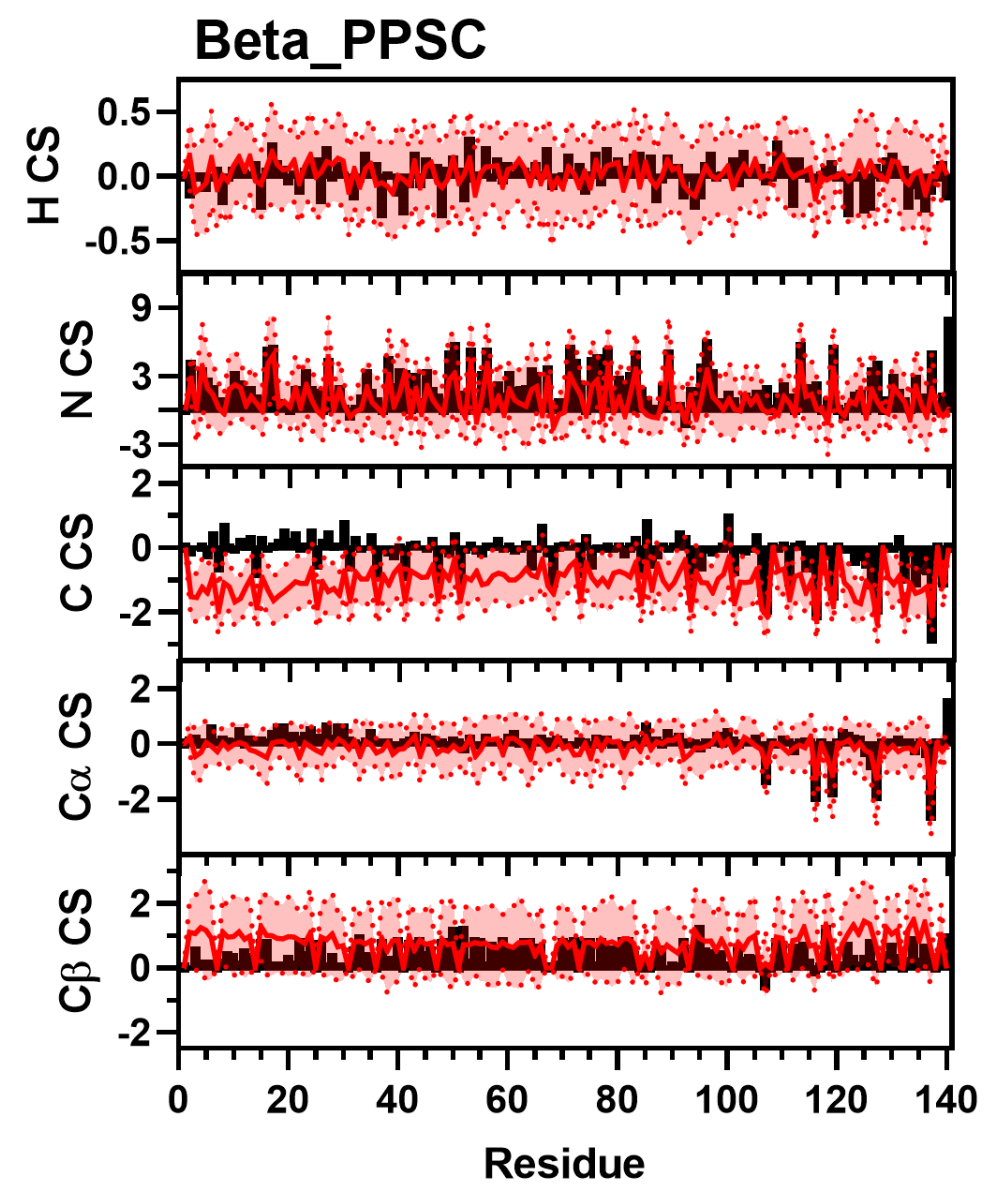

Figure S41: Comparison of Simulated and Experimental NMR Chemical Shift Data. Simulated Chemical Shift values from the Beta_PPSC ensemble (red) overlayed on experimental data (black bars) of N (Top), H (Upper Middle), C (Middle), C $\alpha$ (Lower Middle), and C $\beta$ (Bottom) chemical shifts from Sung et al. ${ }^{8}$ Neighbor corrected random coil chemical shift values have been subtracted from both simulated and experimental data ${ }^{11}$. 


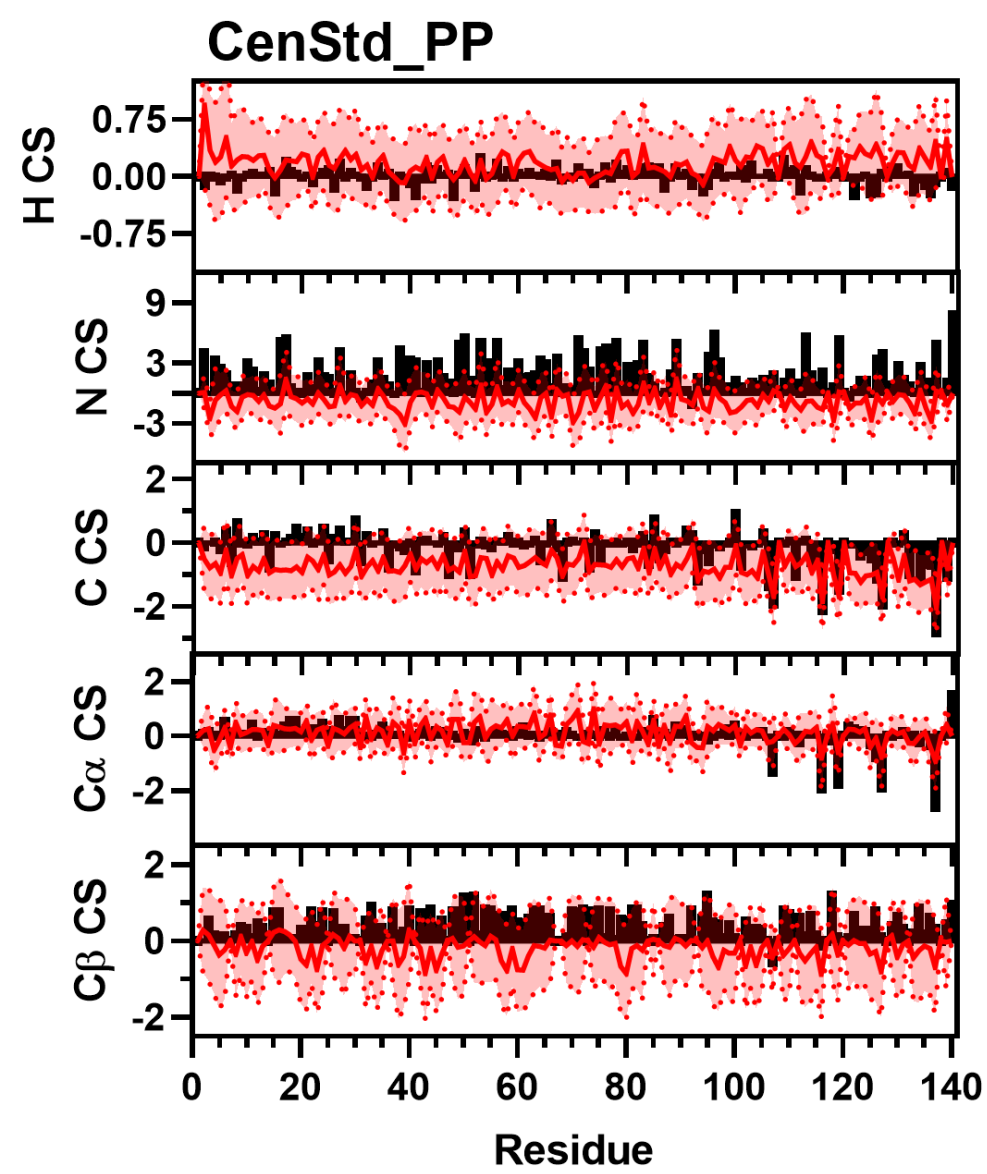

Figure S42: Comparison of Simulated and Experimental NMR Chemical Shift Data. Simulated Chemical Shift values from the CenStd_PP ensemble (red) overlayed on experimental data (black bars) of N (Top), H (Upper Middle), C (Middle), C $\alpha$ (Lower Middle), and C $\beta$ (Bottom) chemical shifts from Sung et al. ${ }^{8}$ Neighbor corrected random coil chemical shift values have been subtracted from both simulated and experimental data ${ }^{11}$. 


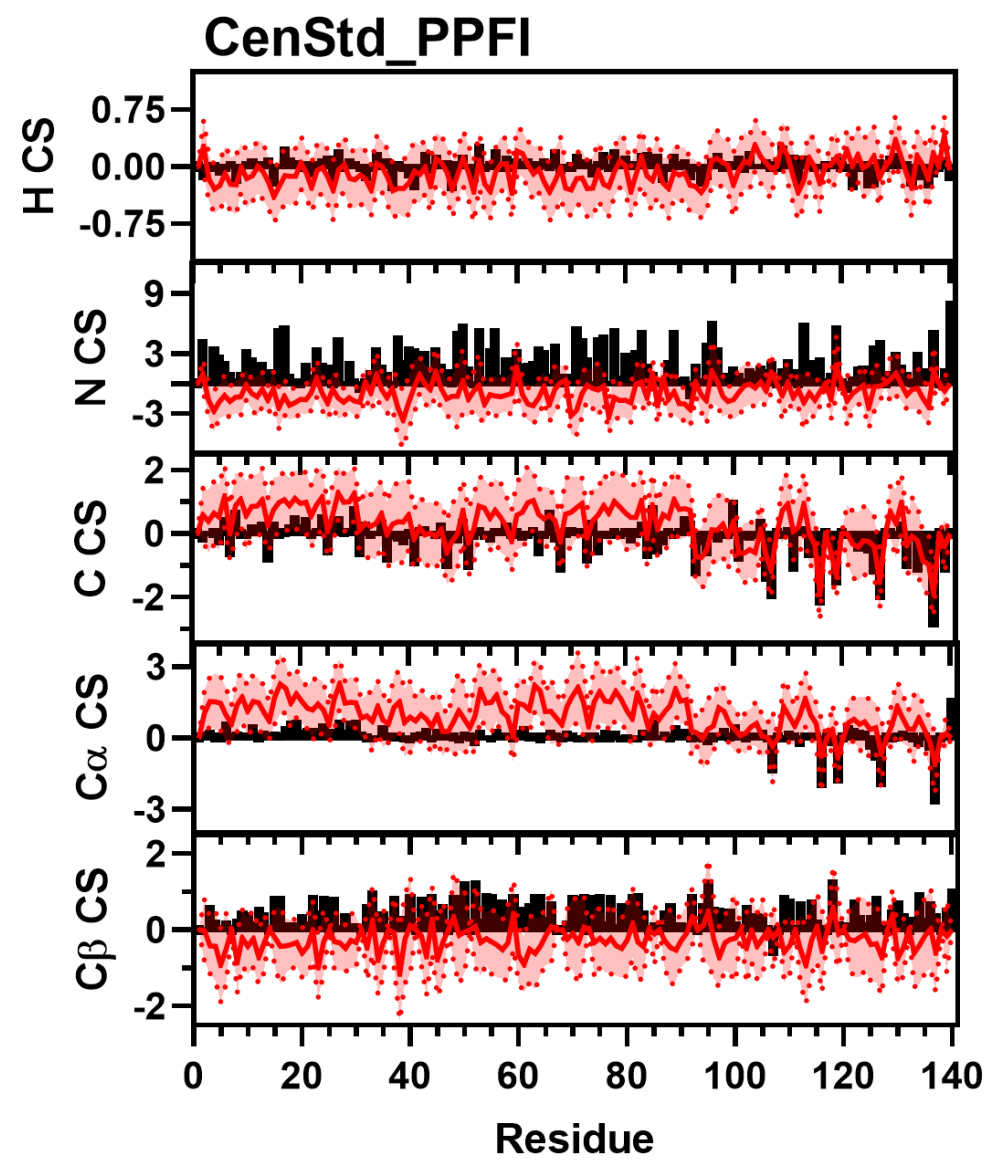

Figure S43: Comparison of Simulated and Experimental NMR Chemical Shift Data. Simulated Chemical Shift values from the CenStd_PPFI ensemble (red) overlayed on experimental data (black bars) of N (Top), H (Upper Middle), C (Middle), C $\alpha$ (Lower Middle), and C $\beta$ (Bottom) chemical shifts from Sung et al. ${ }^{8}$ Neighbor corrected random coil chemical shift values have been subtracted from both simulated and experimental data ${ }^{11}$. 


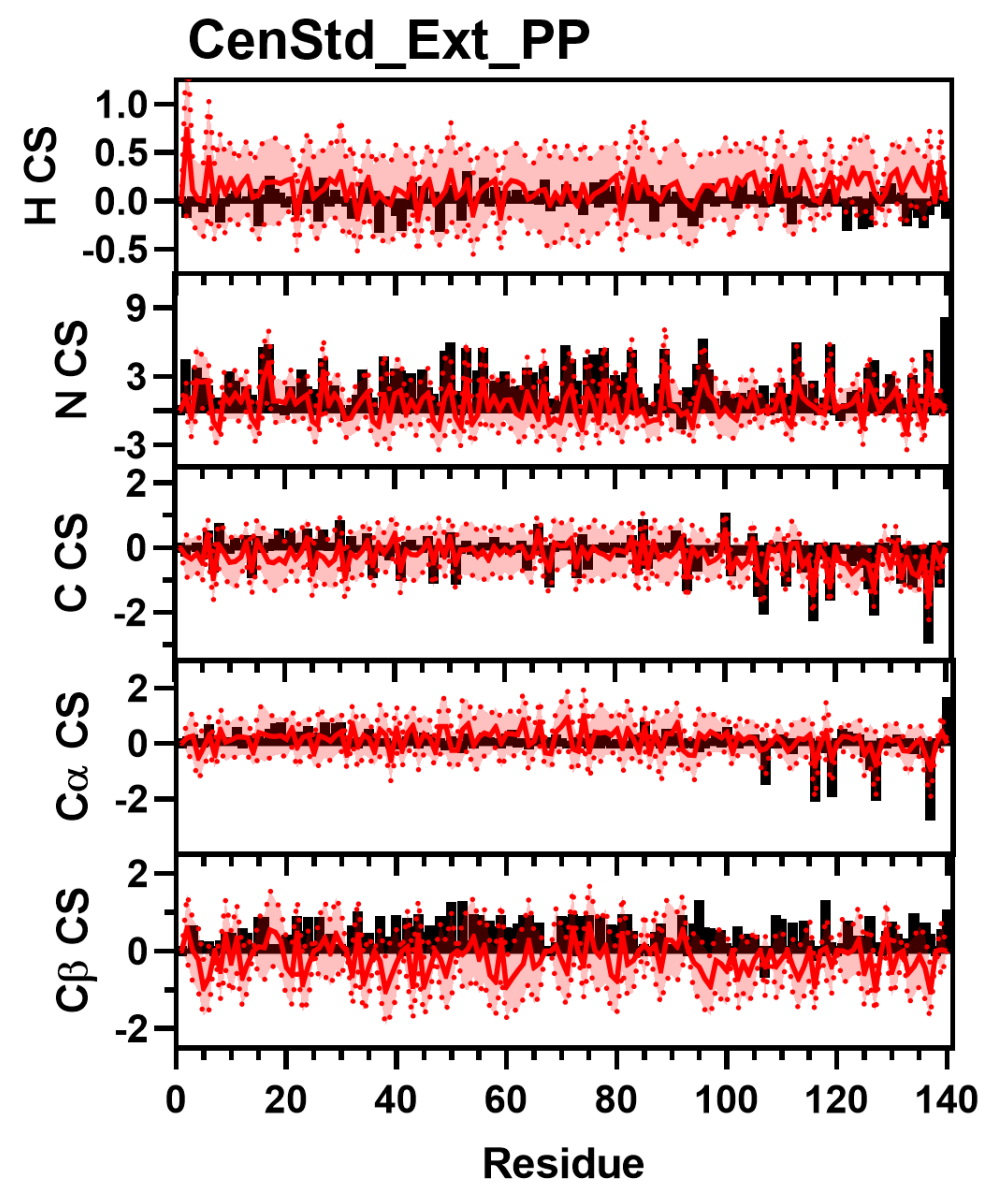

Figure S44: Comparison of Simulated and Experimental NMR Chemical Shift Data. Simulated Chemical Shift values from the CenStd_Ext_PP ensemble (red) overlayed on experimental data (black bars) of N (Top), H (Upper Middle), C (Middle), C $\alpha$ (Lower Middle), and C $\beta$ (Bottom) chemical shifts from Sung et al. ${ }^{8}$ Neighbor corrected random coil chemical shift values have been subtracted from both simulated and experimental data ${ }^{11}$. 


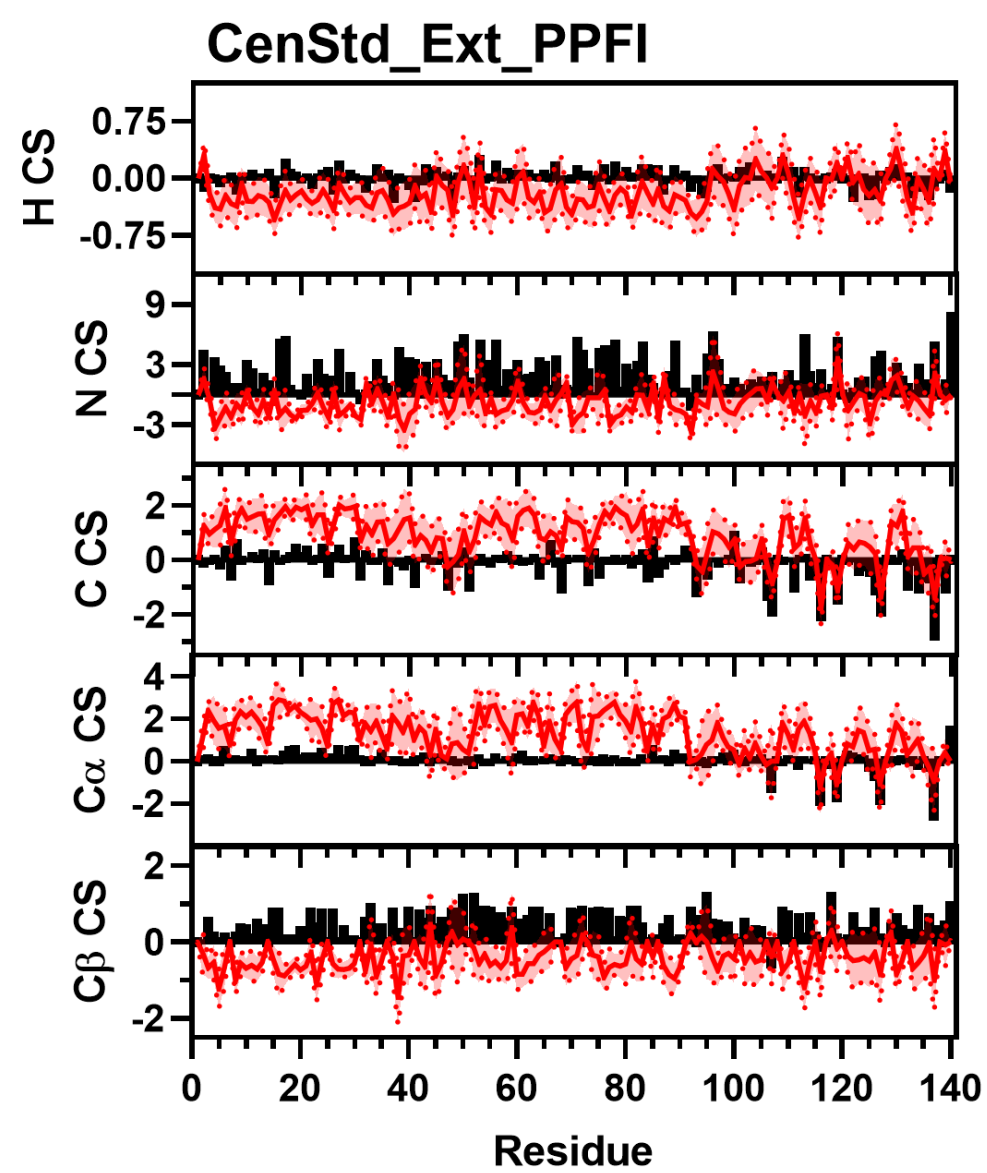

Figure S45: Comparison of Simulated and Experimental NMR Chemical Shift Data. Simulated Chemical Shift values from the CenStd_Ext_PPFI ensemble (red) overlayed on experimental data (black bars) of N (Top), H (Upper Middle), C (Middle), C $\alpha$ (Lower Middle), and C $\beta$ (Bottom) chemical shifts from Sung et al. ${ }^{8}$ Neighbor corrected random coil chemical shift values have been subtracted from both simulated and experimental data ${ }^{11}$. 


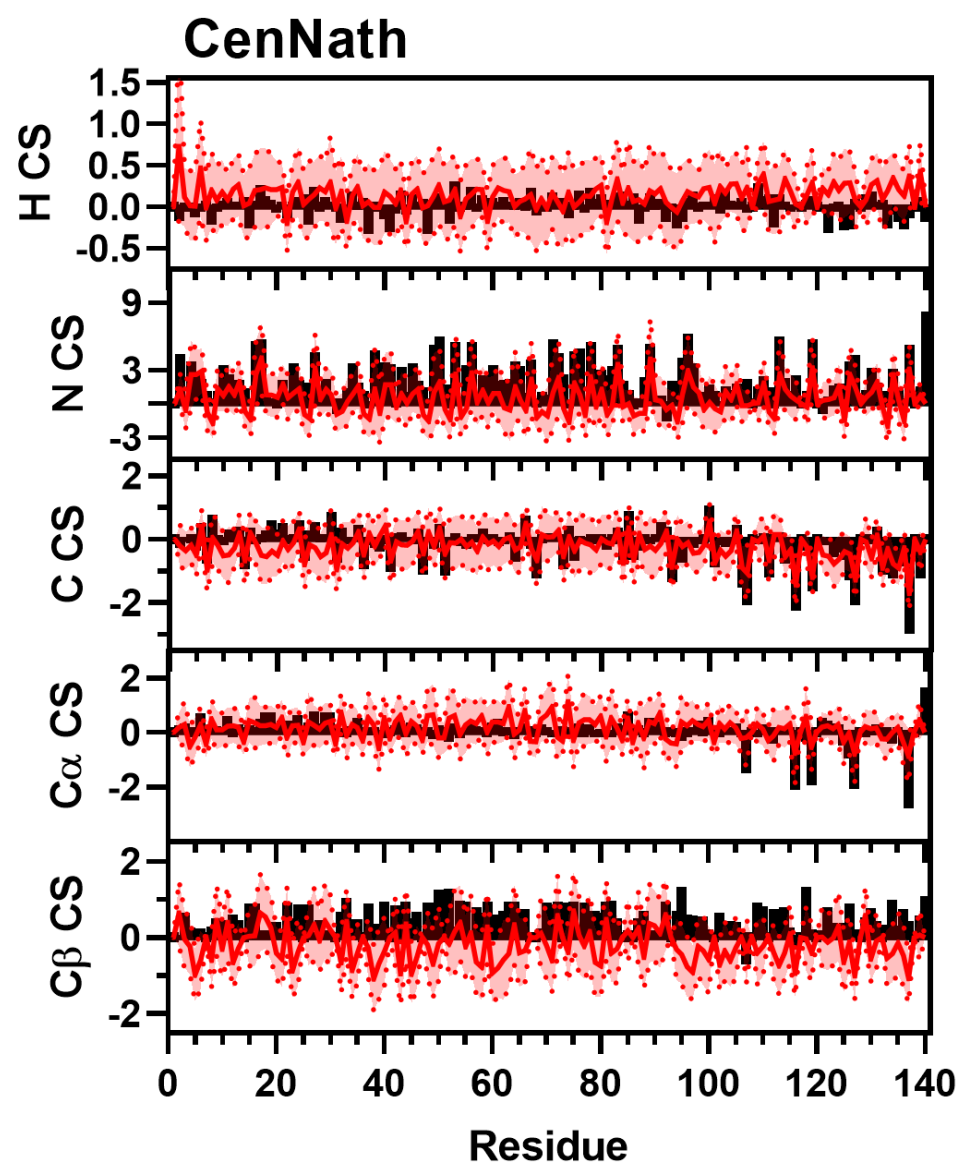

Figure S46: Comparison of Simulated and Experimental NMR Chemical Shift Data. Simulated Chemical Shift values from the CenNath ensemble (red) overlayed on experimental data (black bars) of N (Top), H (Upper Middle), C (Middle), C $\alpha$ (Lower Middle), and C $\beta$ (Bottom) chemical shifts from Sung et al. ${ }^{8}$ Neighbor corrected random coil chemical shift values have been subtracted from both simulated and experimental data ${ }^{11}$. 


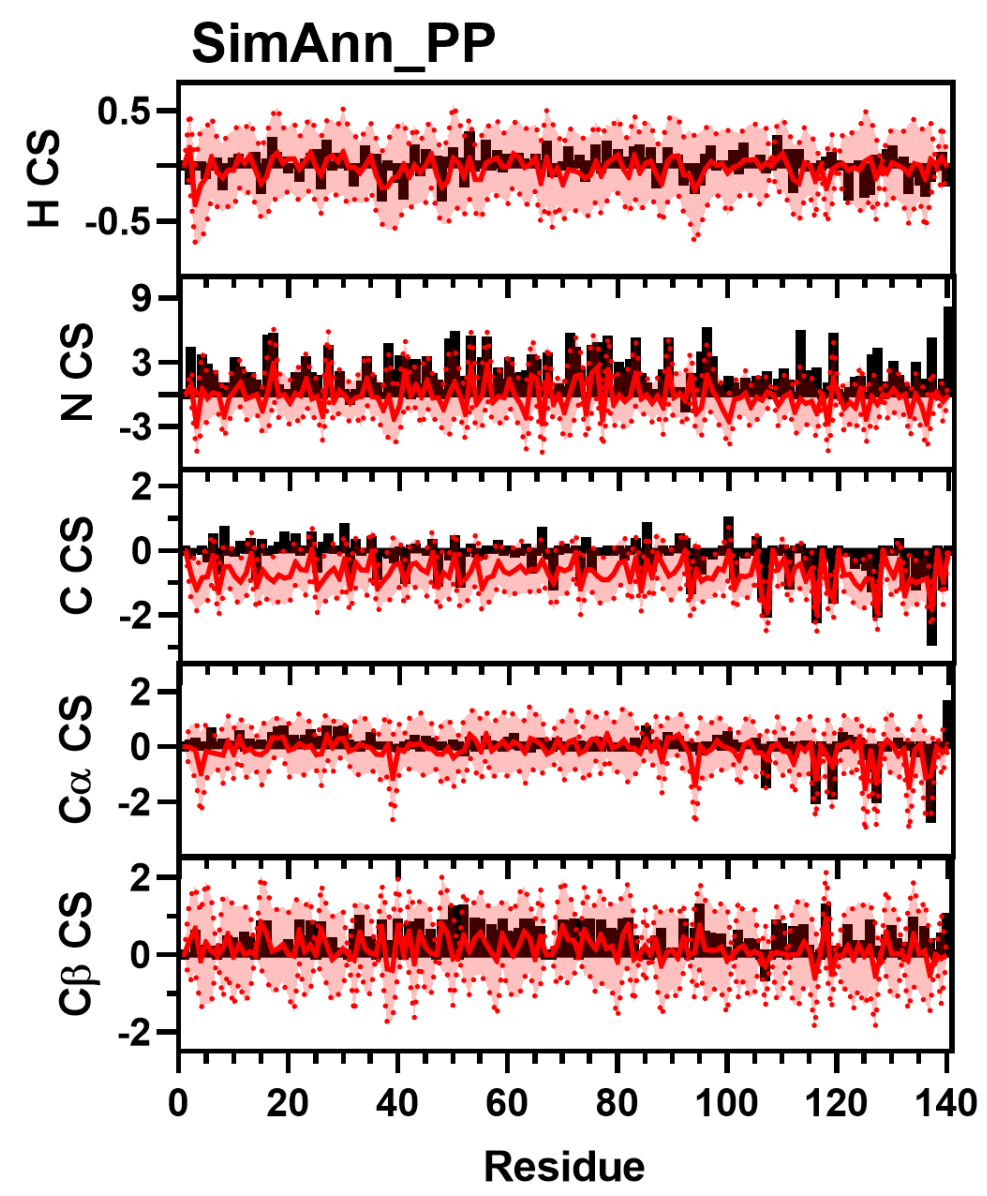

Figure S47: Comparison of Simulated and Experimental NMR Chemical Shift Data. Simulated Chemical Shift values from the SimAnn_PP ensemble (red) overlayed on experimental data (black bars) of N (Top), H (Upper Middle), C (Middle), C $\alpha$ (Lower Middle), and C $\beta$ (Bottom) chemical shifts from Sung et al. ${ }^{8}$ Neighbor corrected random coil chemical shift values have been subtracted from both simulated and experimental data ${ }^{11}$. 


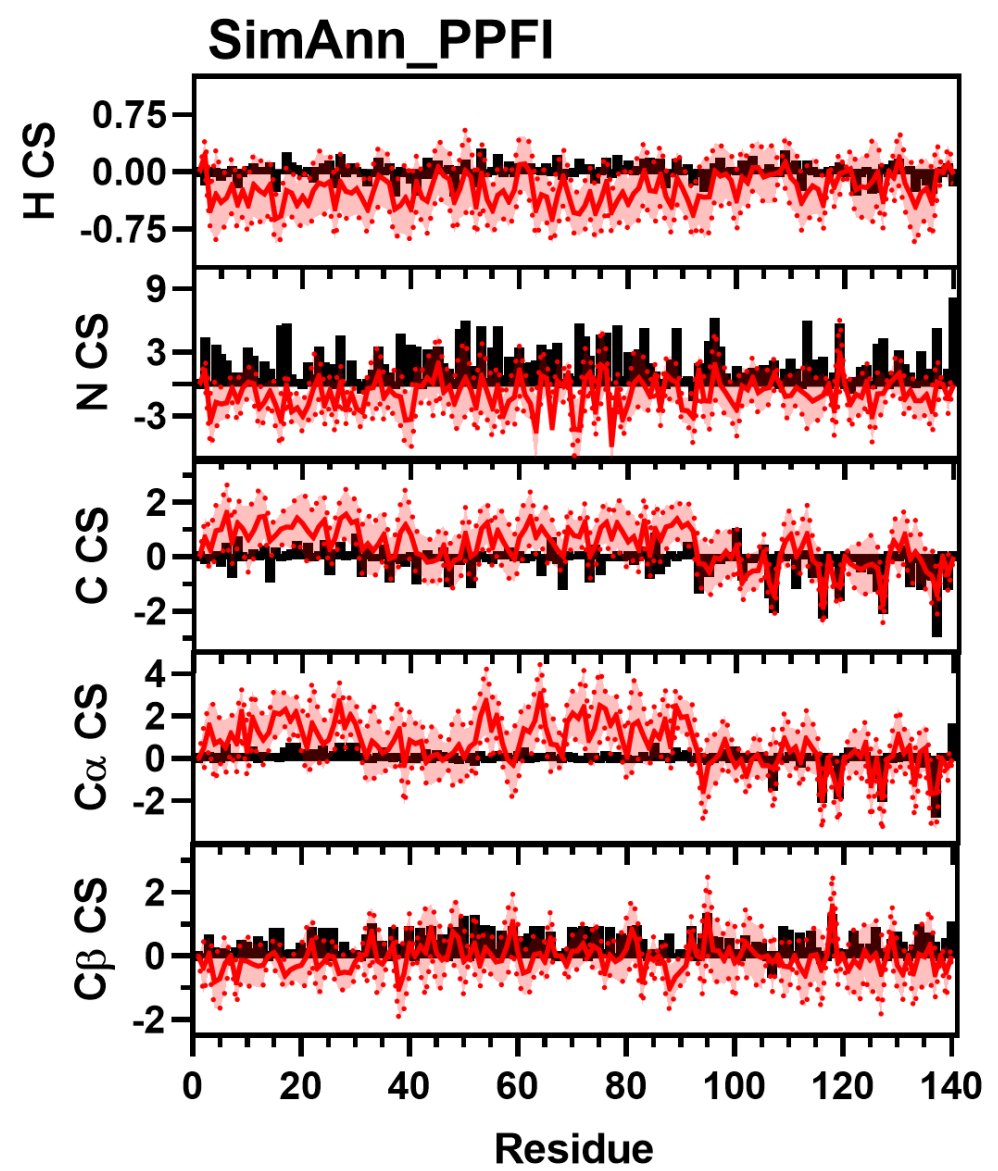

Figure S48: Comparison of Simulated and Experimental NMR Chemical Shift Data. Simulated Chemical Shift values from the SimAnn_PPFI ensemble (red) overlayed on experimental data (black bars) of N (Top), H (Upper Middle), C (Middle), C $\alpha$ (Lower Middle), and C $\beta$ (Bottom) chemical shifts from Sung et al. ${ }^{8}$ Neighbor corrected random coil chemical shift values have been subtracted from both simulated and experimental data ${ }^{11}$. 


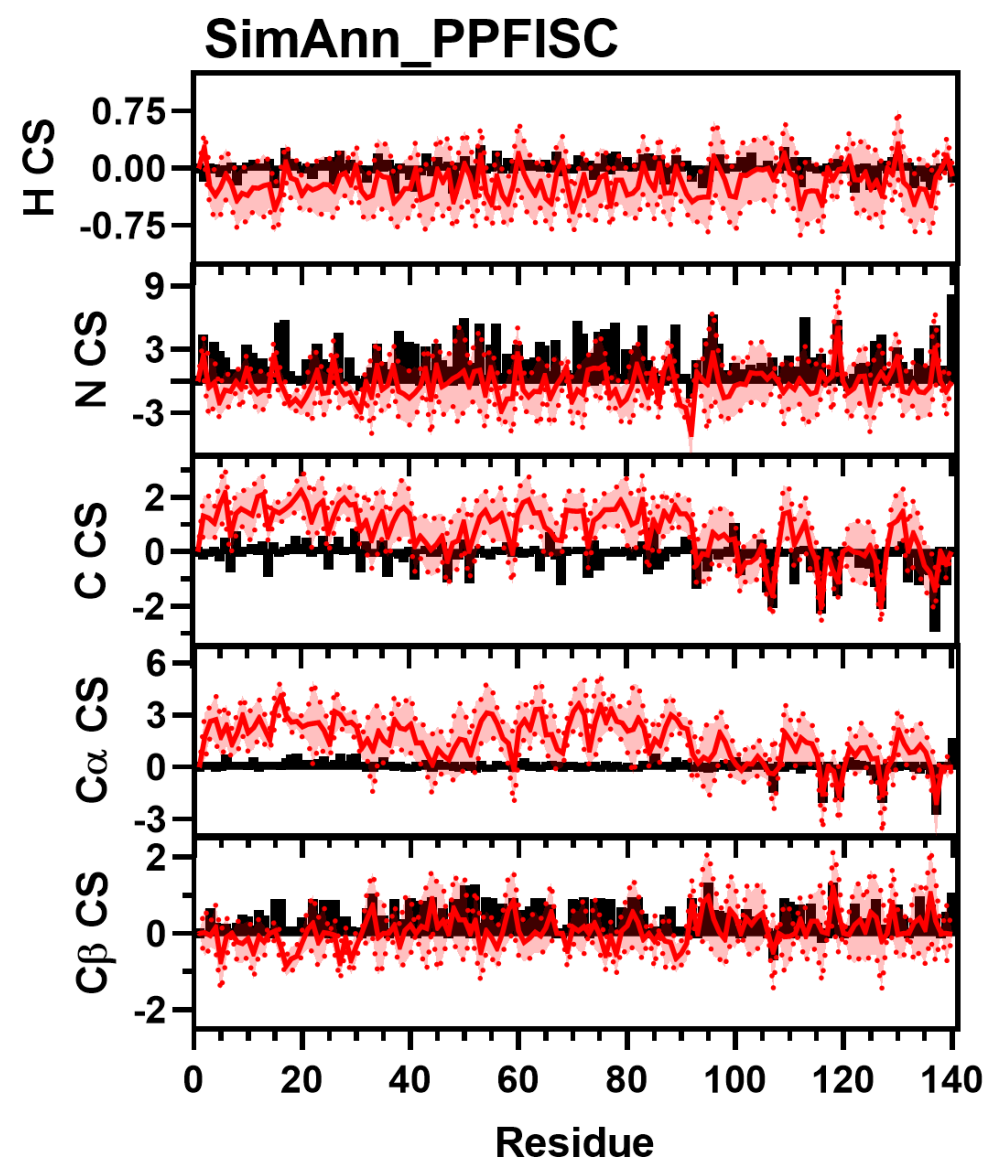

Figure S49: Comparison of Simulated and Experimental NMR Chemical Shift Data. Simulated Chemical Shift values from the SimAnn_PPFISC ensemble (red) overlayed on experimental data (black bars) of N (Top), H (Upper Middle), C (Middle), C $\alpha$ (Lower Middle), and C $\beta$ (Bottom) chemical shifts from Sung et al. ${ }^{8}$ Neighbor corrected random coil chemical shift values have been subtracted from both simulated and experimental data ${ }^{11}$. 


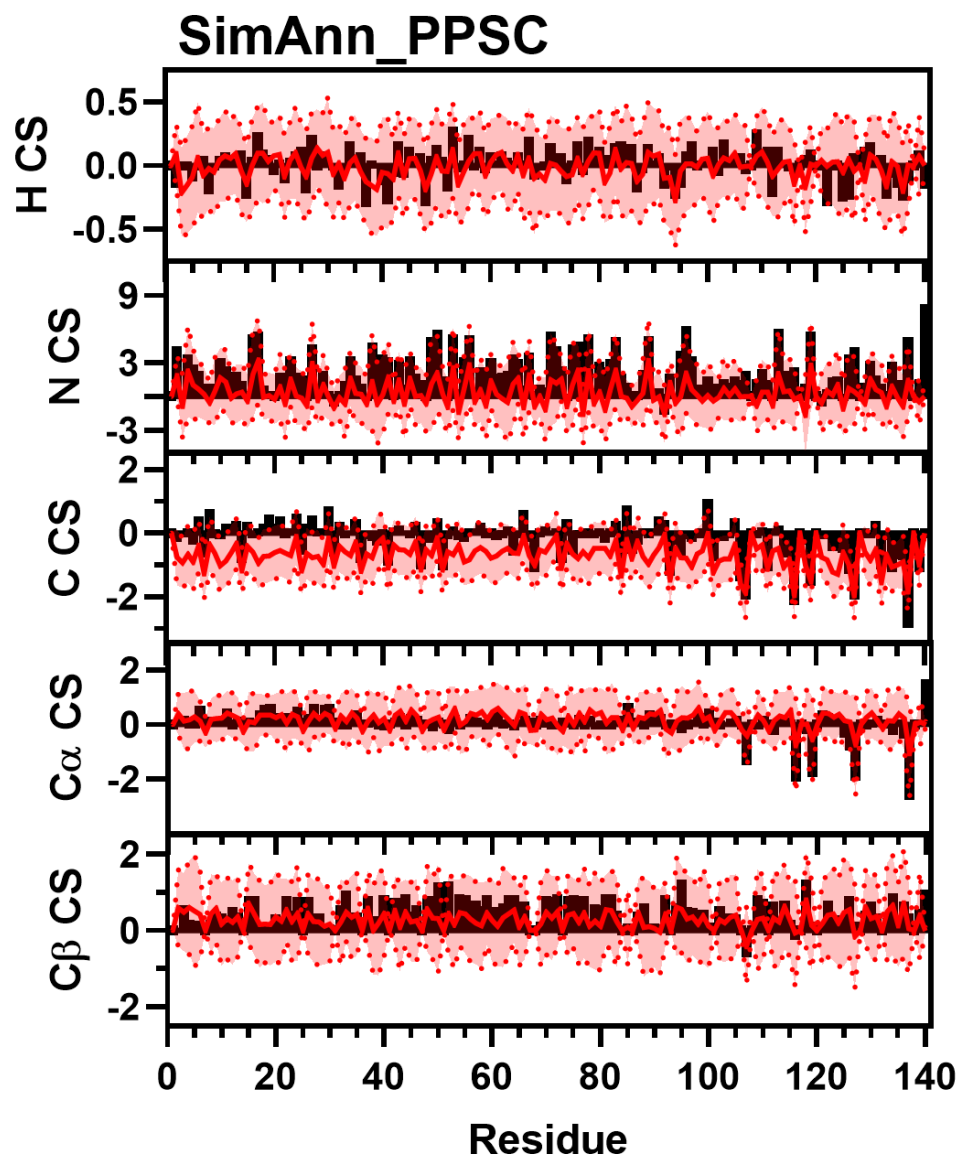

Figure S50: Comparison of Simulated and Experimental NMR Chemical Shift Data. Simulated Chemical Shift values from the SimAnn_PPSC ensemble (red) overlayed on experimental data (black bars) of $\mathrm{N}$ (Top), $\mathrm{H}$ (Upper Middle), C (Middle), $\mathrm{C} \alpha$ (Lower Middle), and C $\beta$ (Bottom) chemical shifts from Sung et al. ${ }^{8}$ Neighbor corrected random coil chemical shift values have been subtracted from both simulated and experimental data ${ }^{11}$. 


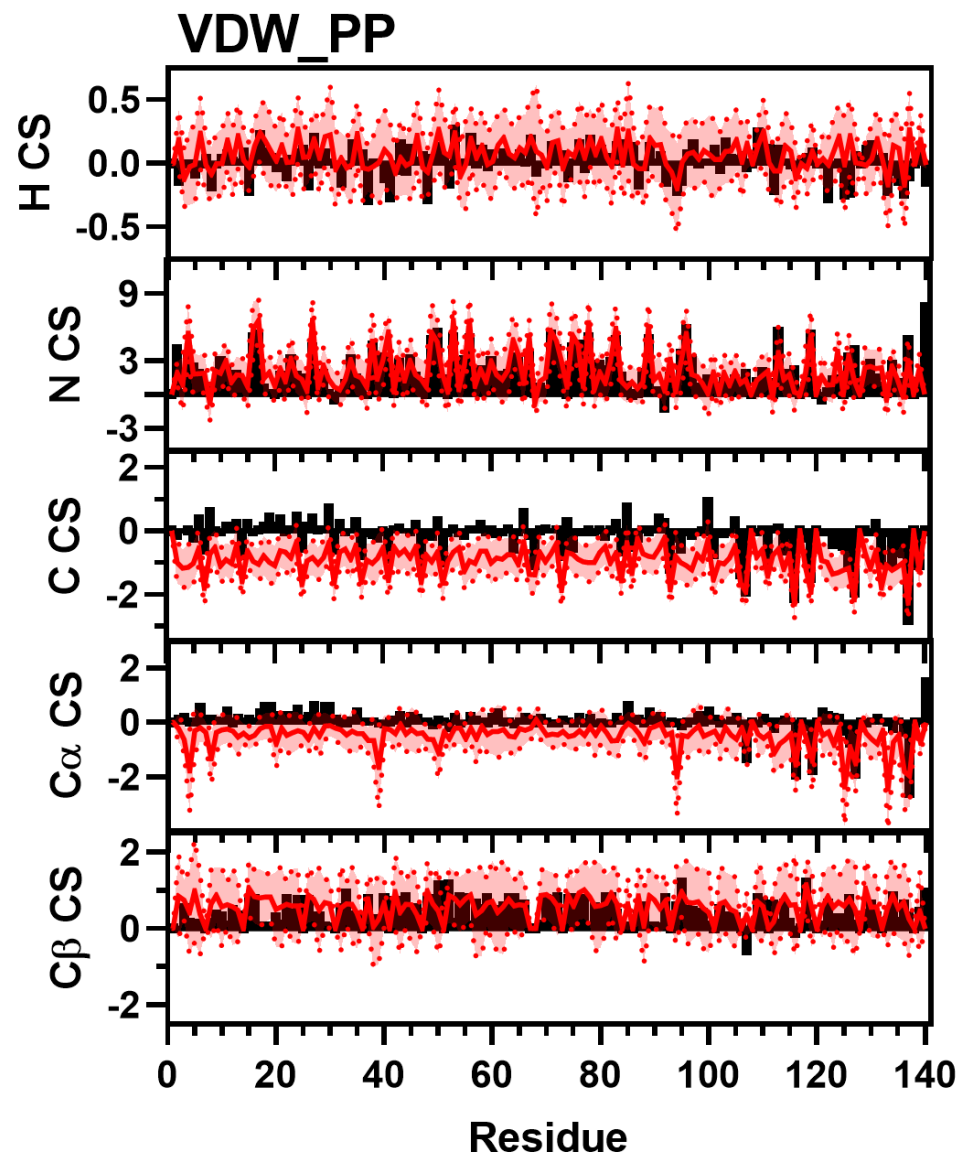

Figure S51: Comparison of Simulated and Experimental NMR Chemical Shift Data. Simulated Chemical Shift values from the VDW_PP ensemble (red) overlayed on experimental data (black bars) of $\mathrm{N}$ (Top), H (Upper Middle), C (Middle), C $\alpha$ (Lower Middle), and C $\beta$ (Bottom) chemical shifts from Sung et al. ${ }^{8}$ Neighbor corrected random coil chemical shift values have been subtracted from both simulated and experimental data ${ }^{11}$. 


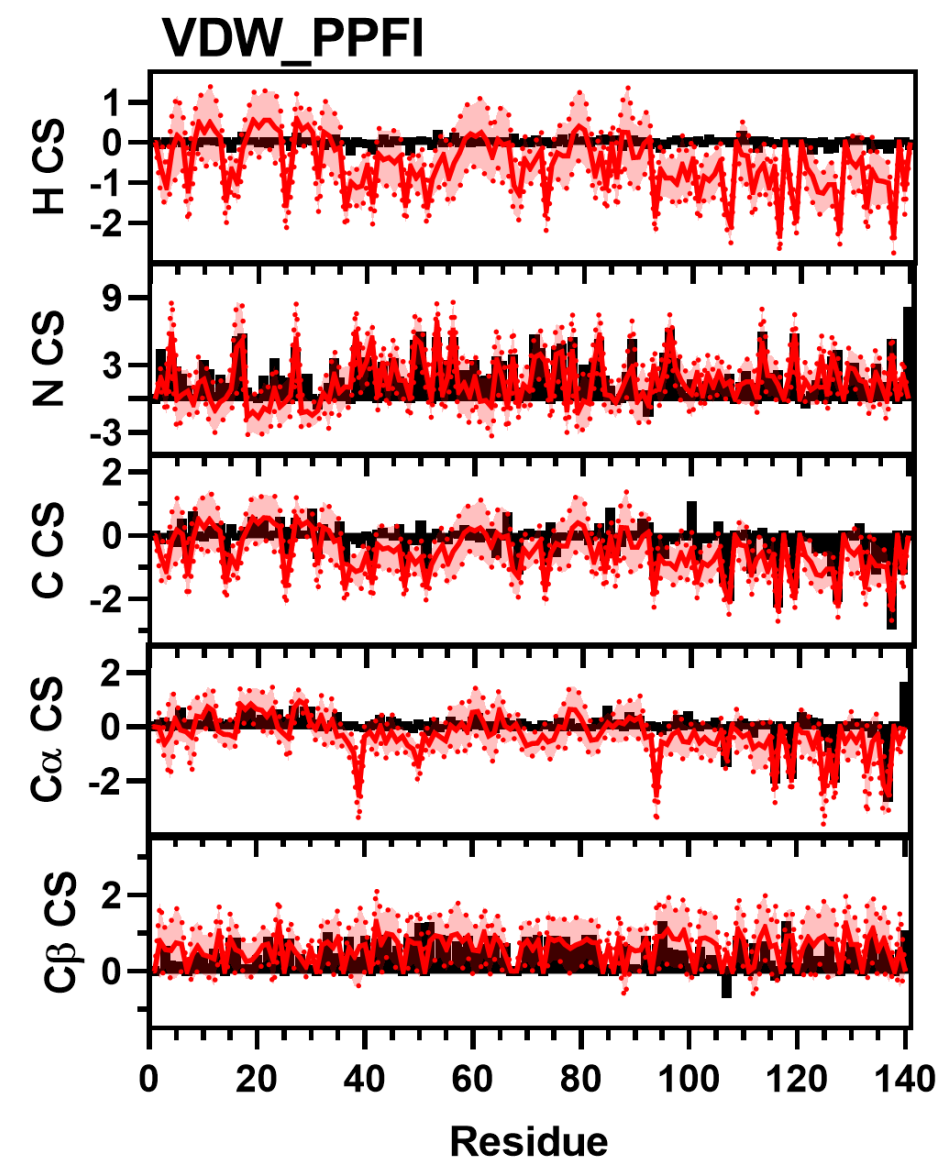

Figure S52: Comparison of Simulated and Experimental NMR Chemical Shift Data. Simulated Chemical Shift values from the VDW_PPFI ensemble (red) overlayed on experimental data (black bars) of N (Top), H (Upper Middle), $\overline{\mathrm{C}}$ (Middle), $\mathrm{C} \alpha$ (Lower Middle), and C $\beta$ (Bottom) chemical shifts from Sung et al. ${ }^{8}$ Neighbor corrected random coil chemical shift values have been subtracted from both simulated and experimental data ${ }^{11}$. 


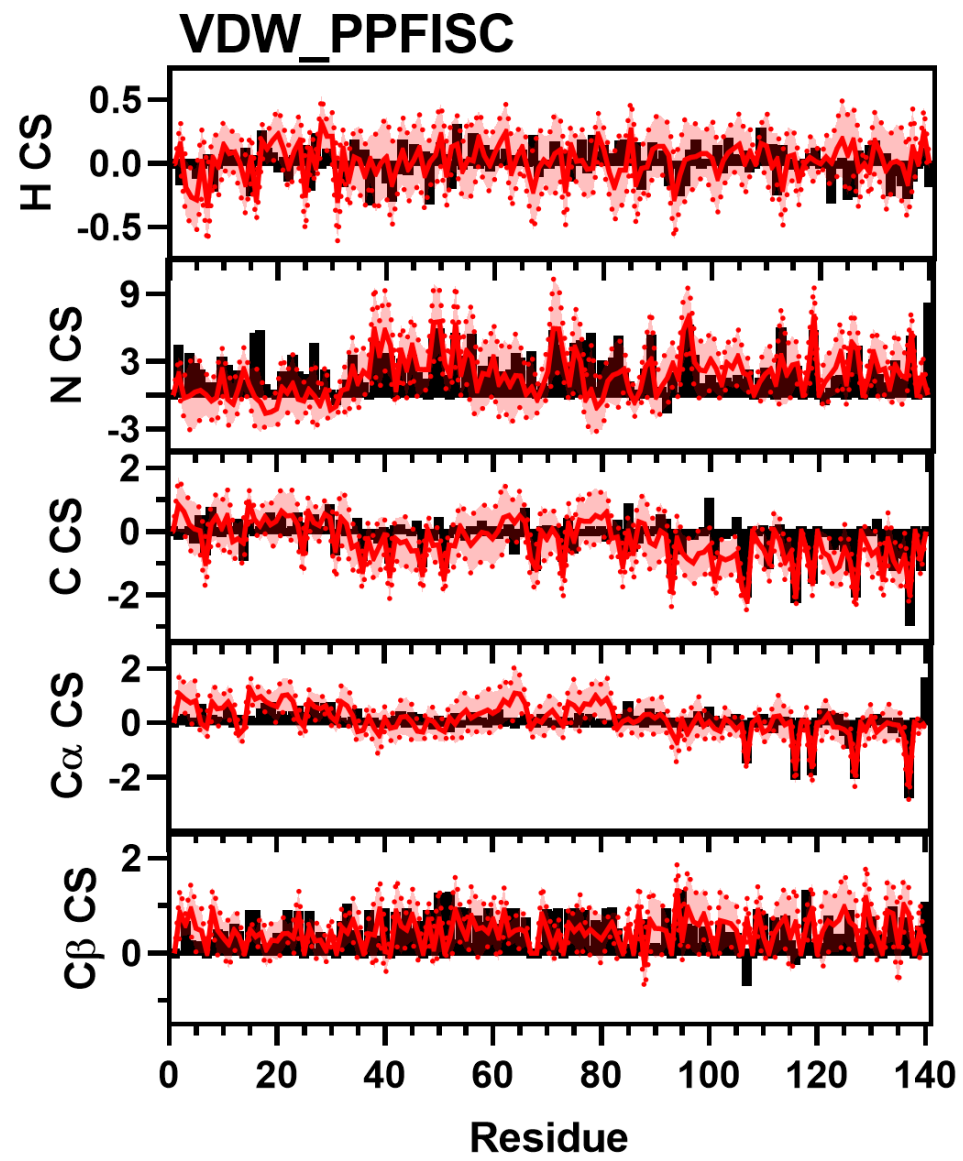

Figure S53: Comparison of Simulated and Experimental NMR Chemical Shift Data. Simulated Chemical Shift values from the VDW_PPFISC ensemble (red) overlayed on experimental data (black bars) of N (Top), H (Upper Middle), C (Middle), C $\alpha$ (Lower Middle), and C $\beta$ (Bottom) chemical shifts from Sung et al. ${ }^{8}$ Neighbor corrected random coil chemical shift values have been subtracted from both simulated and experimental data ${ }^{11}$. 


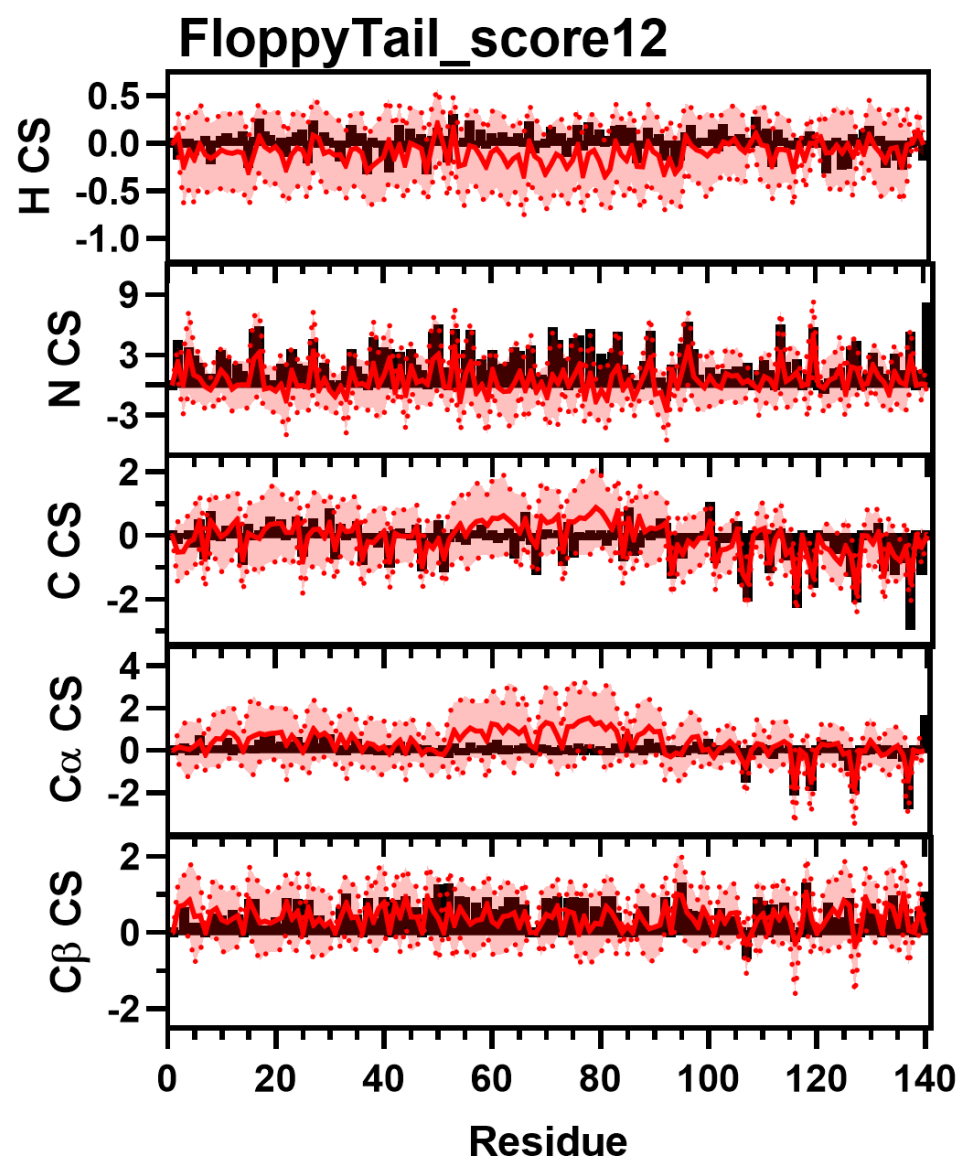

Figure S54: Comparison of Simulated and Experimental NMR Chemical Shift Data. Simulated Chemical Shift values from the FloppyTail_score12 ensemble (red) overlayed on experimental data (black bars) of N (Top), H (Upper Middle), C (Middle), C $\alpha$ (Lower Middle), and C $\beta$ (Bottom) chemical shifts from Sung et al. ${ }^{8}$ Neighbor corrected random coil chemical shift values have been subtracted from both simulated and experimental data ${ }^{11}$. 


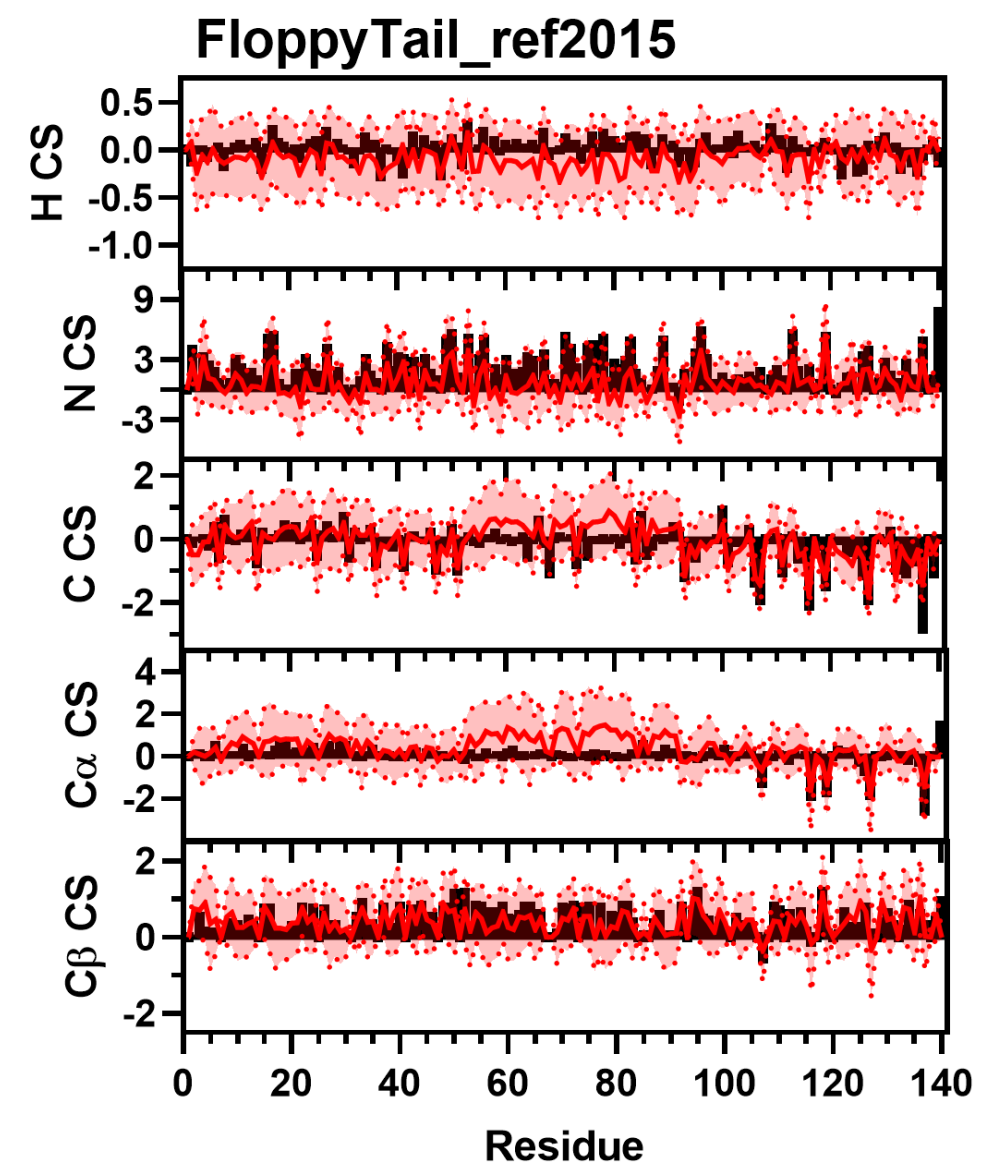

Figure S55: Comparison of Simulated and Experimental NMR Chemical Shift Data. Simulated Chemical Shift values from the FloppyTail_ref2015 ensemble (red) overlayed on experimental data (black bars) of N (Top), H (Upper Middle), C (Middle), C $\alpha$ (Lower Middle), and C $\beta$ (Bottom) chemical shifts from Sung et al. ${ }^{8}$ Neighbor corrected random coil chemical shift values have been subtracted from both simulated and experimental data ${ }^{11}$. 


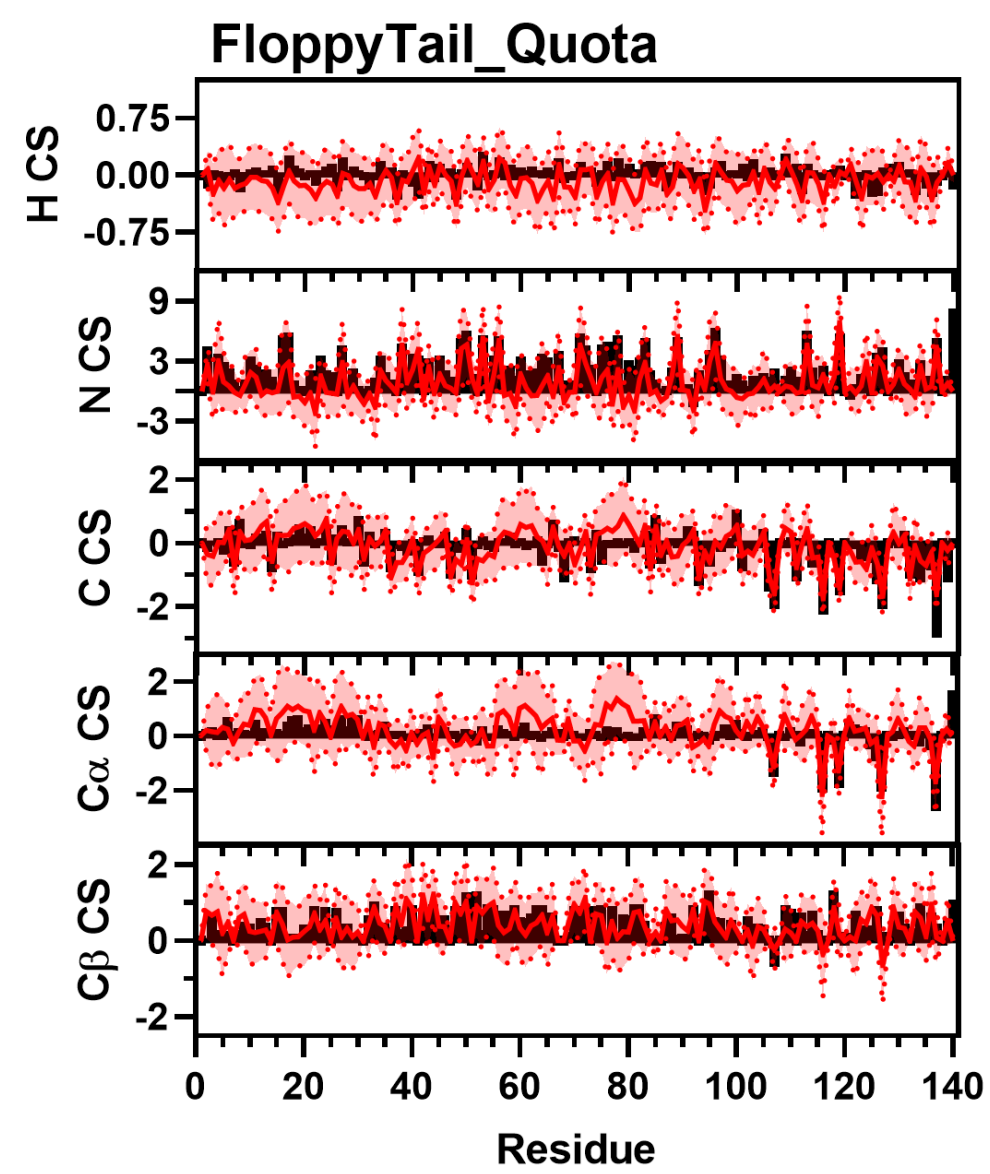

Figure S56: Comparison of Simulated and Experimental NMR Chemical Shift Data. Simulated Chemical Shift values from the FloppyTail_Quota ensemble (red) overlayed on experimental data (black bars) of N (Top), H (Upper Middle), C (Middle), C $\alpha$ (Lower Middle), and C $\beta$ (Bottom) chemical shifts from Sung et al. ${ }^{8}$ Neighbor corrected random coil chemical shift values have been subtracted from both simulated and experimental data ${ }^{11}$. 


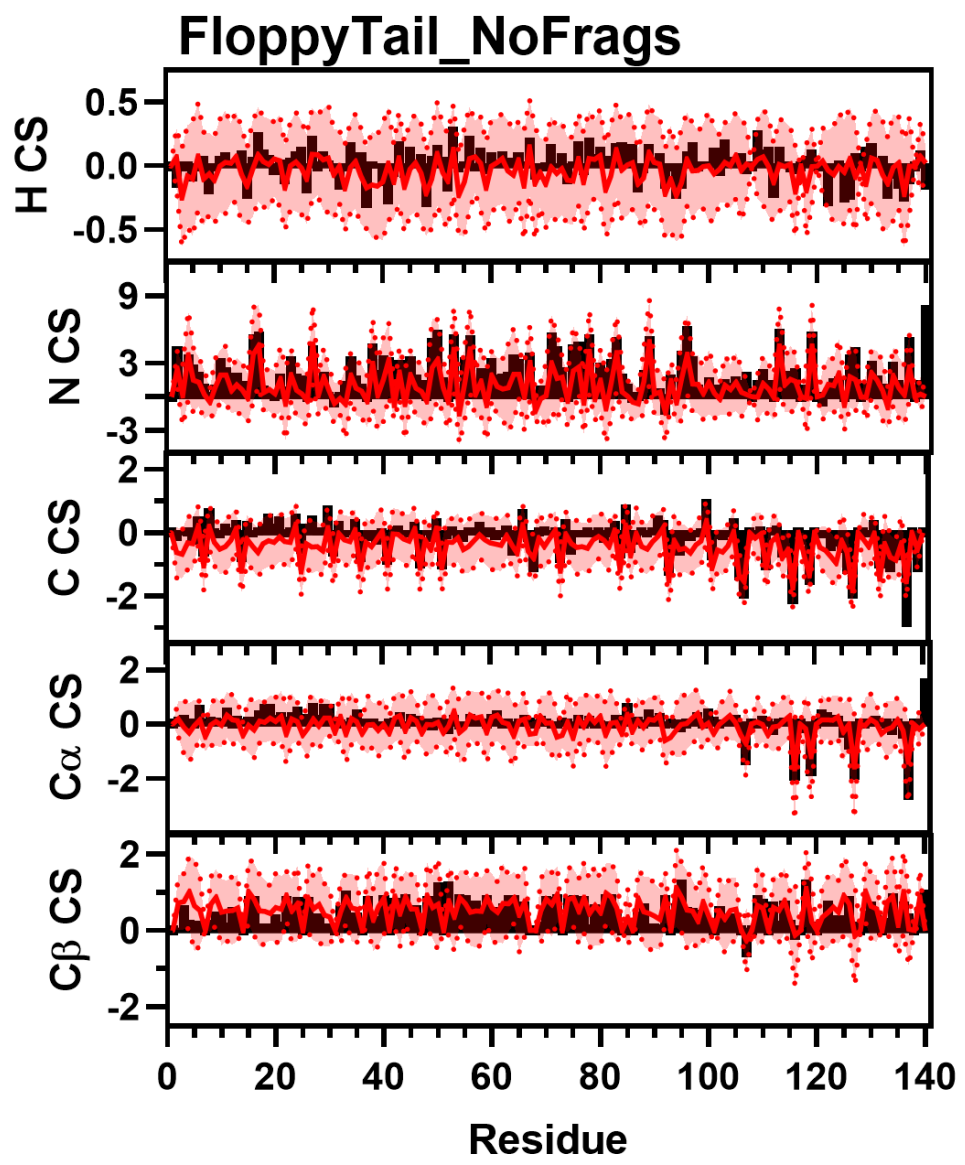

Figure S57: Comparison of Simulated and Experimental NMR Chemical Shift Data. Simulated Chemical Shift values from the FloppyTail_NoFrags ensemble (red) overlayed on experimental data (black bars) of N (Top), H (Upper Middle), C (Middle), C $\alpha$ (Lower Middle), and C $\beta$ (Bottom) chemical shifts from Sung et al. ${ }^{8}$ Neighbor corrected random coil chemical shift values have been subtracted from both simulated and experimental data ${ }^{11}$. 


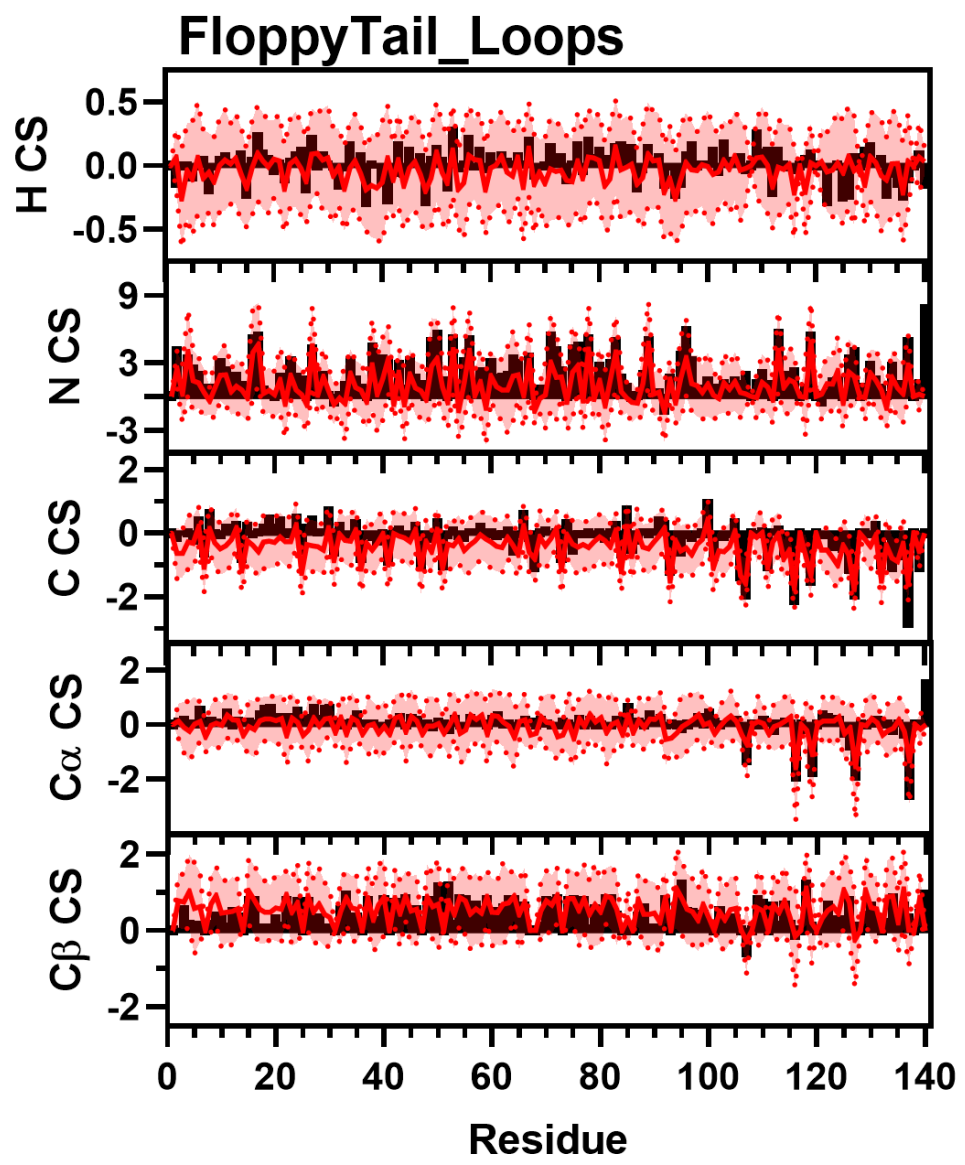

Figure S58: Comparison of Simulated and Experimental NMR Chemical Shift Data. Simulated Chemical Shift values from FloppyTail_Loops ensemble (red) overlayed on experimental data (black bars) of N (Top), H (Upper Middle), C (Middle), C $\alpha$ (Lower Middle), and C $\beta$ (Bottom) chemical shifts from Sung et al. ${ }^{8}$ Neighbor corrected random coil chemical shift values have been subtracted from both simulated and experimental data ${ }^{11}$. 


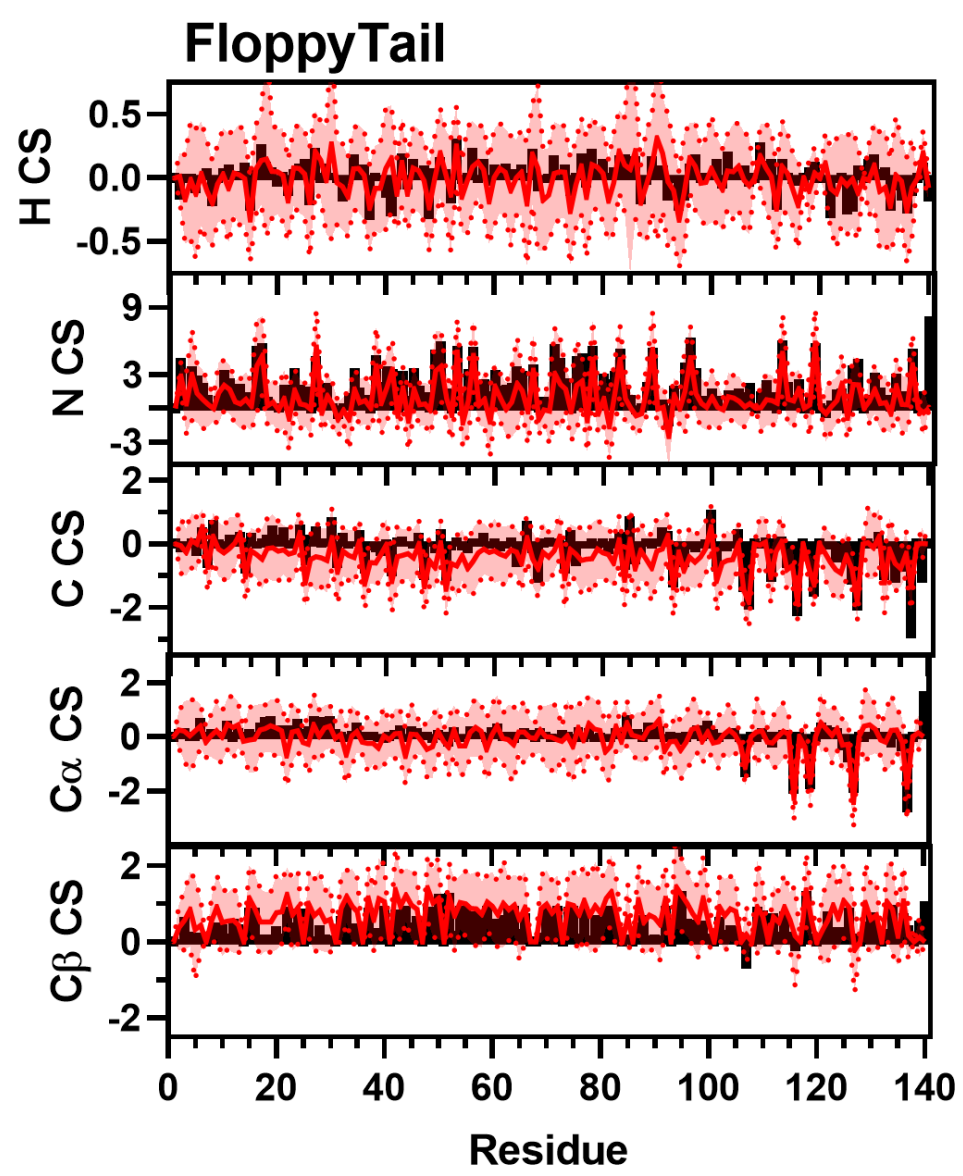

Figure S59: Comparison of Simulated and Experimental NMR Chemical Shift Data. Simulated Chemical Shift values from the FloppyTail ensemble (red) overlayed on experimental data (black bars) of N (Top), H (Upper Middle), C (Middle), C $\alpha$ (Lower Middle), and C $\beta$ (Bottom) chemical shifts from Sung et al. ${ }^{8}$ Neighbor corrected random coil chemical shift values have been subtracted from both simulated and experimental data ${ }^{11}$. 


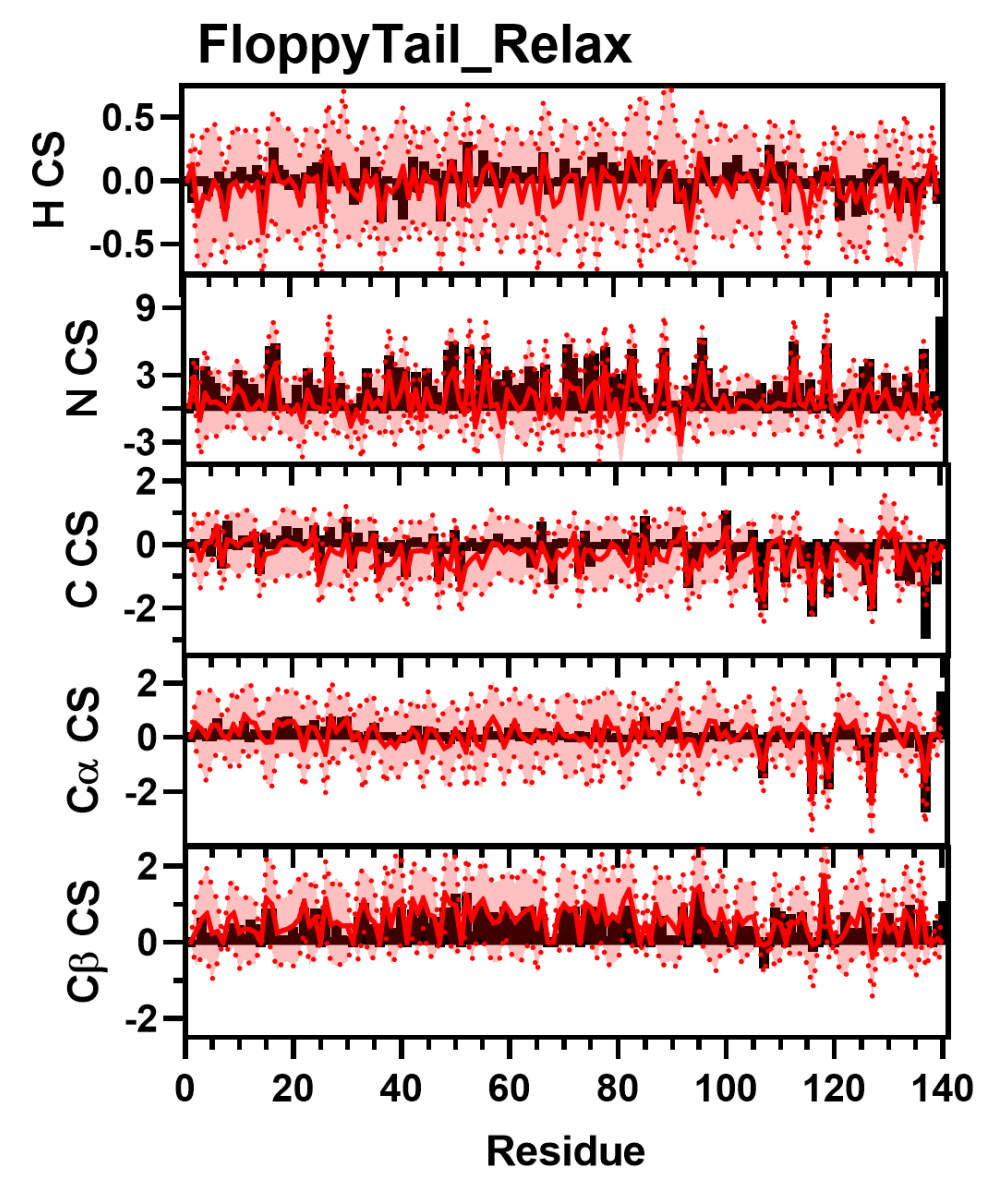

Figure S60: Comparison of Simulated and Experimental NMR Chemical Shift Data. Simulated Chemical Shift values from the FloppyTail_Relax ensemble (red) overlayed on experimental data (black bars) of N (Top), H (Upper Middle), C (Middle), C $\alpha$ (Lower Middle), and C $\beta$ (Bottom) chemical shifts from Sung et al. ${ }^{8}$ Neighbor corrected random coil chemical shift values have been subtracted from both simulated and experimental data ${ }^{11}$. 


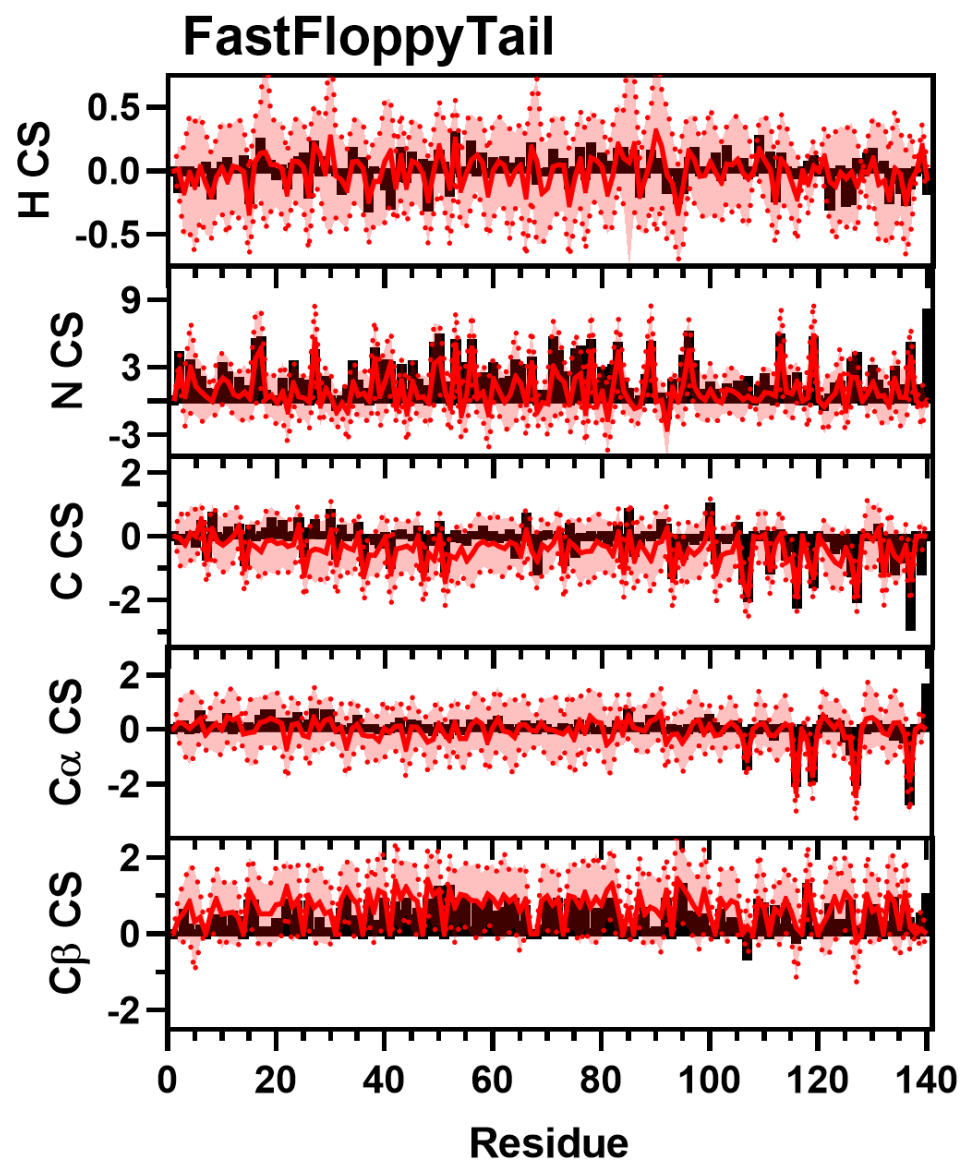

Figure S61: Comparison of Simulated and Experimental NMR Chemical Shift Data. Simulated Chemical Shift values from the FastFloppyTail ensemble (red) overlayed on experimental data (black bars) of $\mathrm{N}$ (Top), H (Upper Middle), C (Middle), C $\alpha$ (Lower Middle), and C $\beta$ (Bottom) chemical shifts from Sung et al. ${ }^{8}$ Neighbor corrected random coil chemical shift values have been subtracted from both simulated and experimental data ${ }^{11}$. 


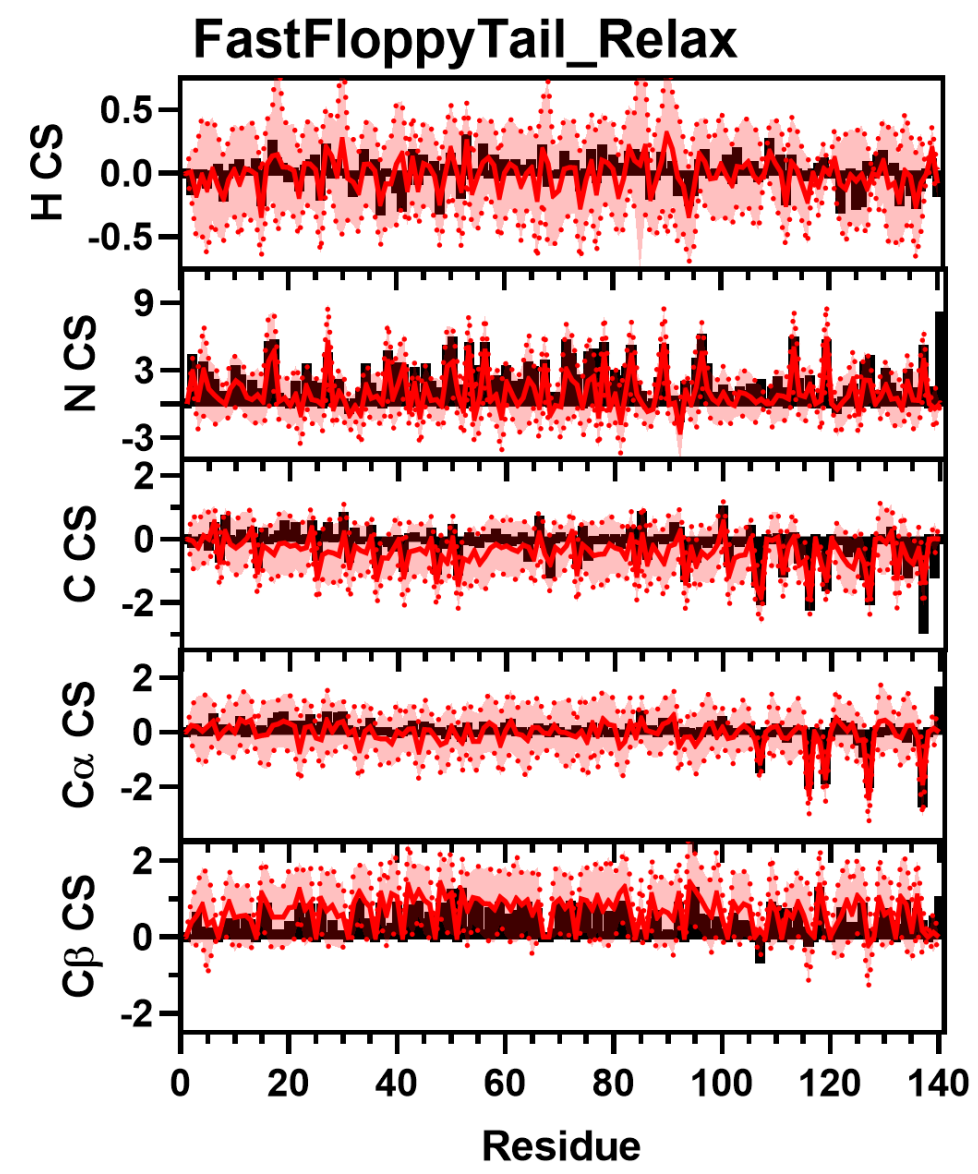

Figure S62: Comparison of Simulated and Experimental NMR Chemical Shift Data. Simulated Chemical Shift values from the FastFloppyTail_Relax ensemble (red) overlayed on experimental data (black bars) of N (Top), H (Upper Middle), C (Middle), C $\alpha$ (Lower Middle), and C $\beta$ (Bottom) chemical shifts from Sung et al. ${ }^{8}$ Neighbor corrected random coil chemical shift values have been subtracted from both simulated and experimental data ${ }^{11}$. 


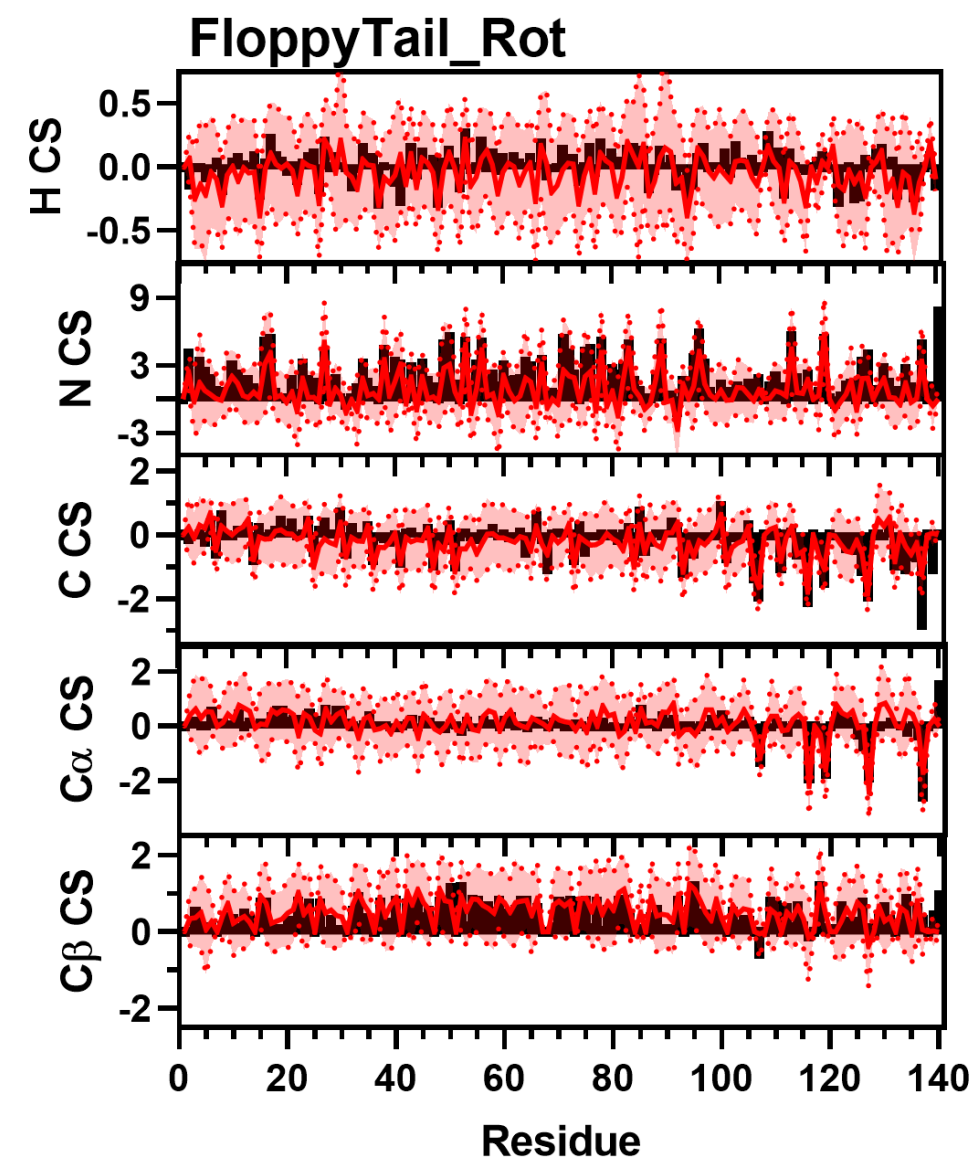

Figure S63: Comparison of Simulated and Experimental NMR Chemical Shift Data. Simulated Chemical Shift values from the FloppyTail_Rot ensemble (red) overlayed on experimental data (black bars) of N (Top), H (Upper Middle), C (Middle), C $\alpha$ (Lower Middle), and C $\beta$ (Bottom) chemical shifts from Sung et al. ${ }^{8}$ Neighbor corrected random coil chemical shift values have been subtracted from both simulated and experimental data ${ }^{11}$. 


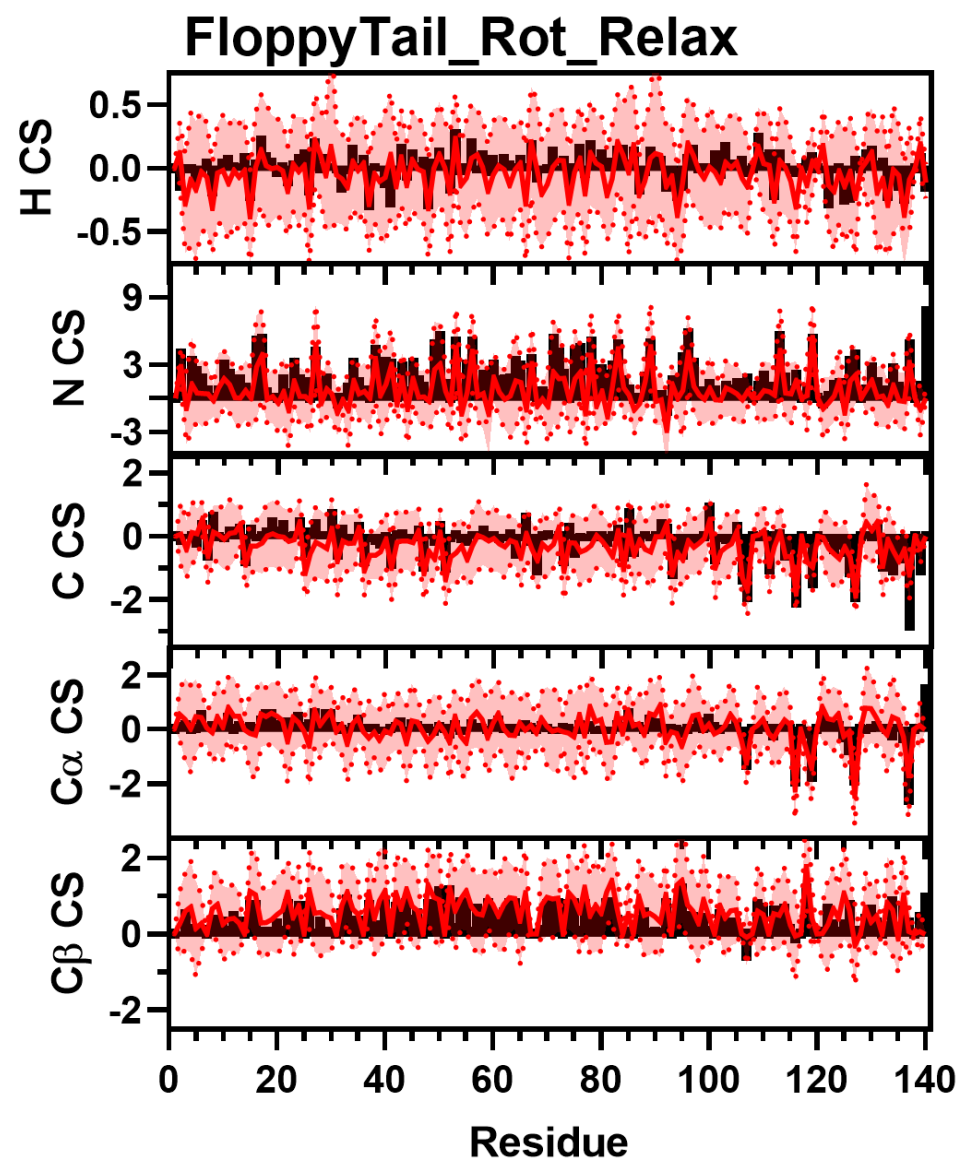

Figure S64: Comparison of Simulated and Experimental NMR Chemical Shift Data. Simulated Chemical Shift values from the FloppyTail_Rot_Relax ensemble (red) overlayed on experimental data (black bars) of N (Top), H (Upper Middle), C (Middle), C $\alpha$ (Lower Middle), and C $\beta$ (Bottom) chemical shifts from Sung et al. ${ }^{8}$ Neighbor corrected random coil chemical shift values have been subtracted from both simulated and experimental data ${ }^{11}$. 


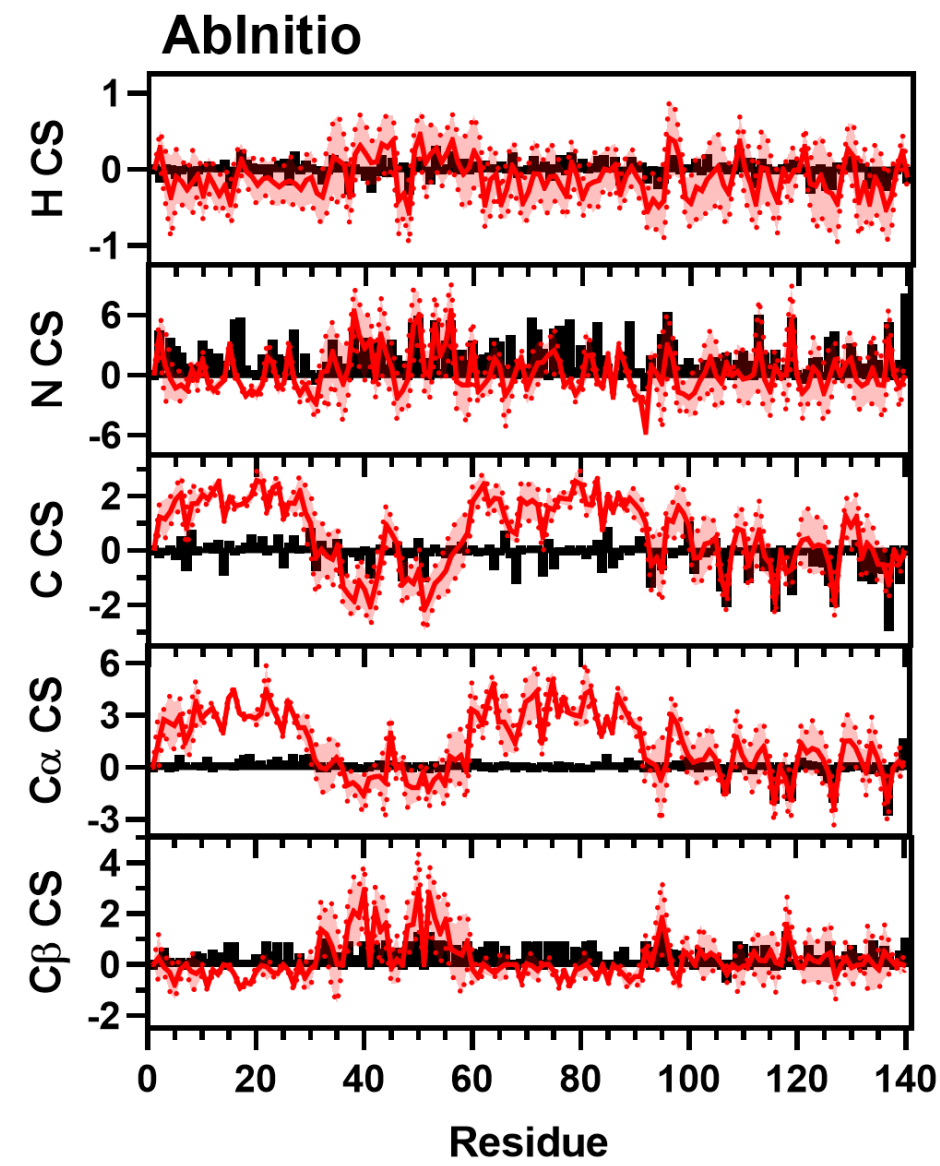

Figure S65: Comparison of Simulated and Experimental NMR Chemical Shift Data. Simulated Chemical Shift values from the AbInitio ensemble (red) overlayed on experimental data (black bars) of $\mathrm{N}$ (Top), H (Upper Middle), C (Middle), C $\alpha$ (Lower Middle), and C $\beta$ (Bottom) chemical shifts from Sung et al. ${ }^{8}$ Neighbor corrected random coil chemical shift values have been subtracted from both simulated and experimental data ${ }^{11}$. 


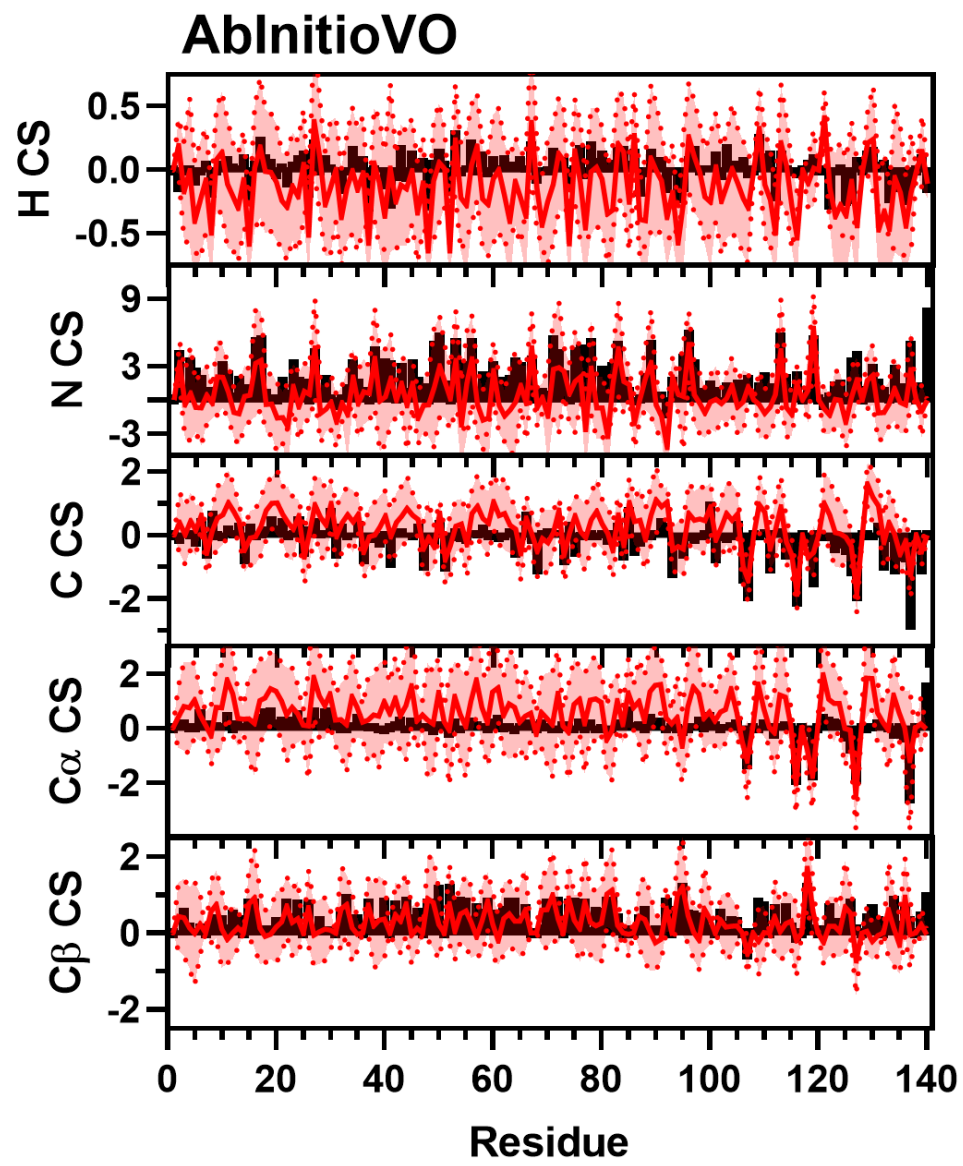

Figure S66: Comparison of Simulated and Experimental NMR Chemical Shift Data. Simulated Chemical Shift values from the AbInitioVO ensemble (red) overlayed on experimental data (black bars) of $\mathrm{N}$ (Top), H (Upper Middle), C (Middle), C $\alpha$ (Lower Middle), and C $\beta$ (Bottom) chemical shifts from Sung et al. ${ }^{8}$ Neighbor corrected random coil chemical shift values have been subtracted from both simulated and experimental data ${ }^{11}$. 

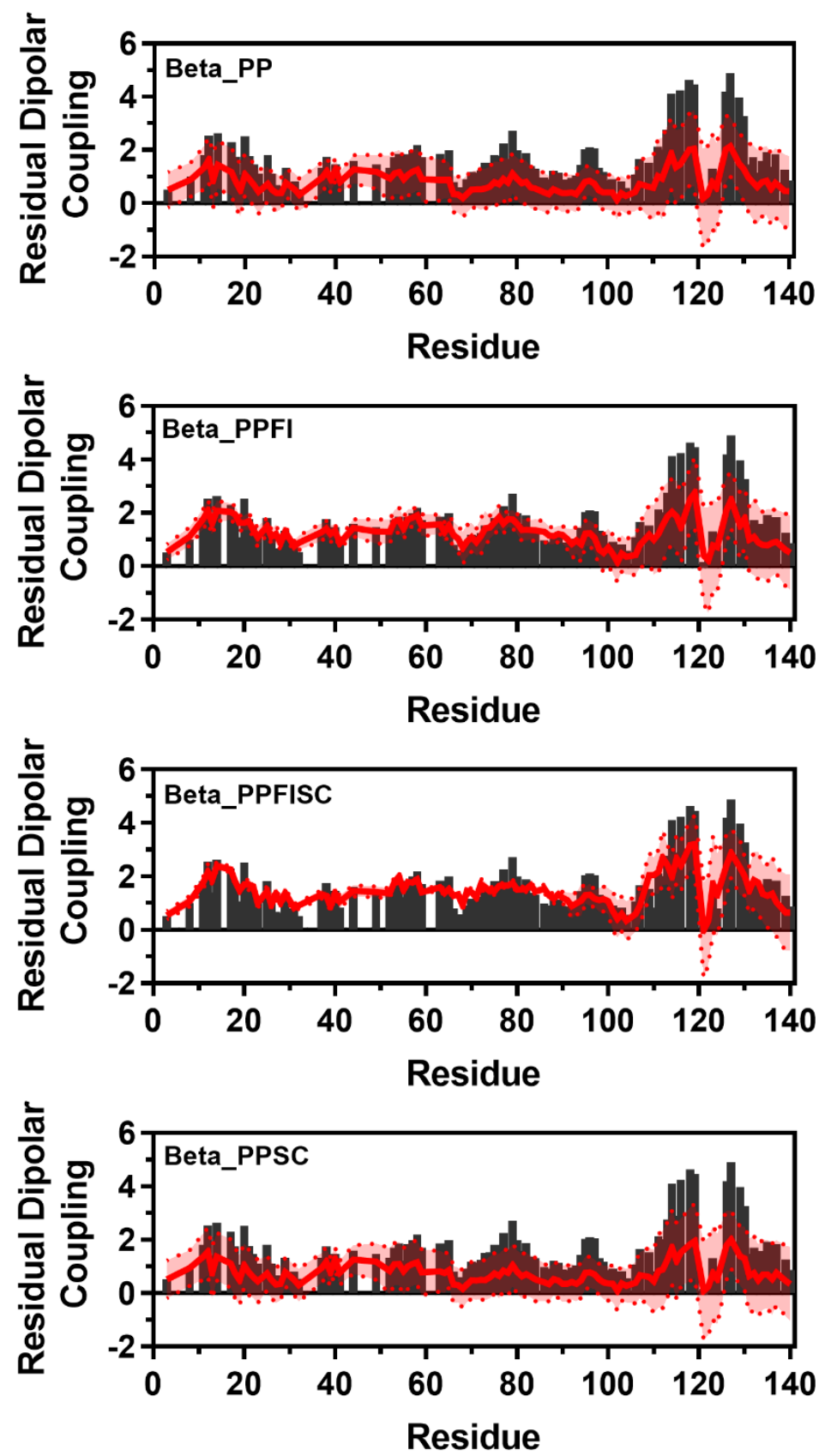

Figure S67: Comparison of Simulated and Experimental Residual Dipolar Coupling Data. Simulated RDC values (red line) from Beta_PP (Top), Beta_PPFI (Upper Middle), Beta_PPFISC (Lower Middle), and Beta_PPSC (Bottom) overlayed on experimental data (black bars) from Bertoncini et al. ${ }^{25}$. 

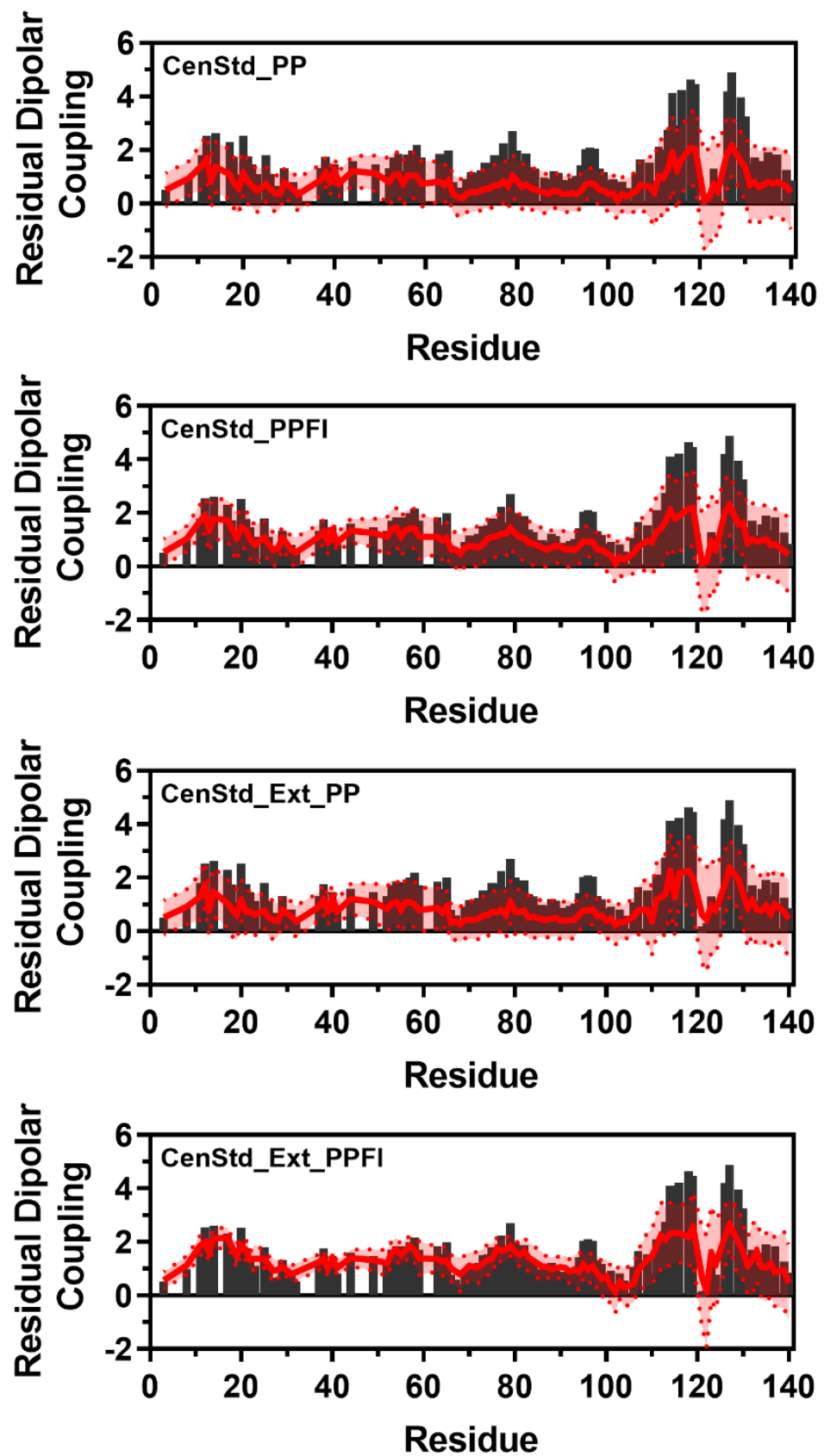

Figure S68: Comparison of Simulated and Experimental Residual Dipolar Coupling Data. Simulated RDC values (red line) from CenStd_PP (Top), CenStd_PPFI (Upper Middle), CenStd_Ext_PP (Lower Middle), and CenStd_Ext_PPFI (Bottom) overlayed on experimental data (black bars) from Bertoncini et al. ${ }^{25}$. 

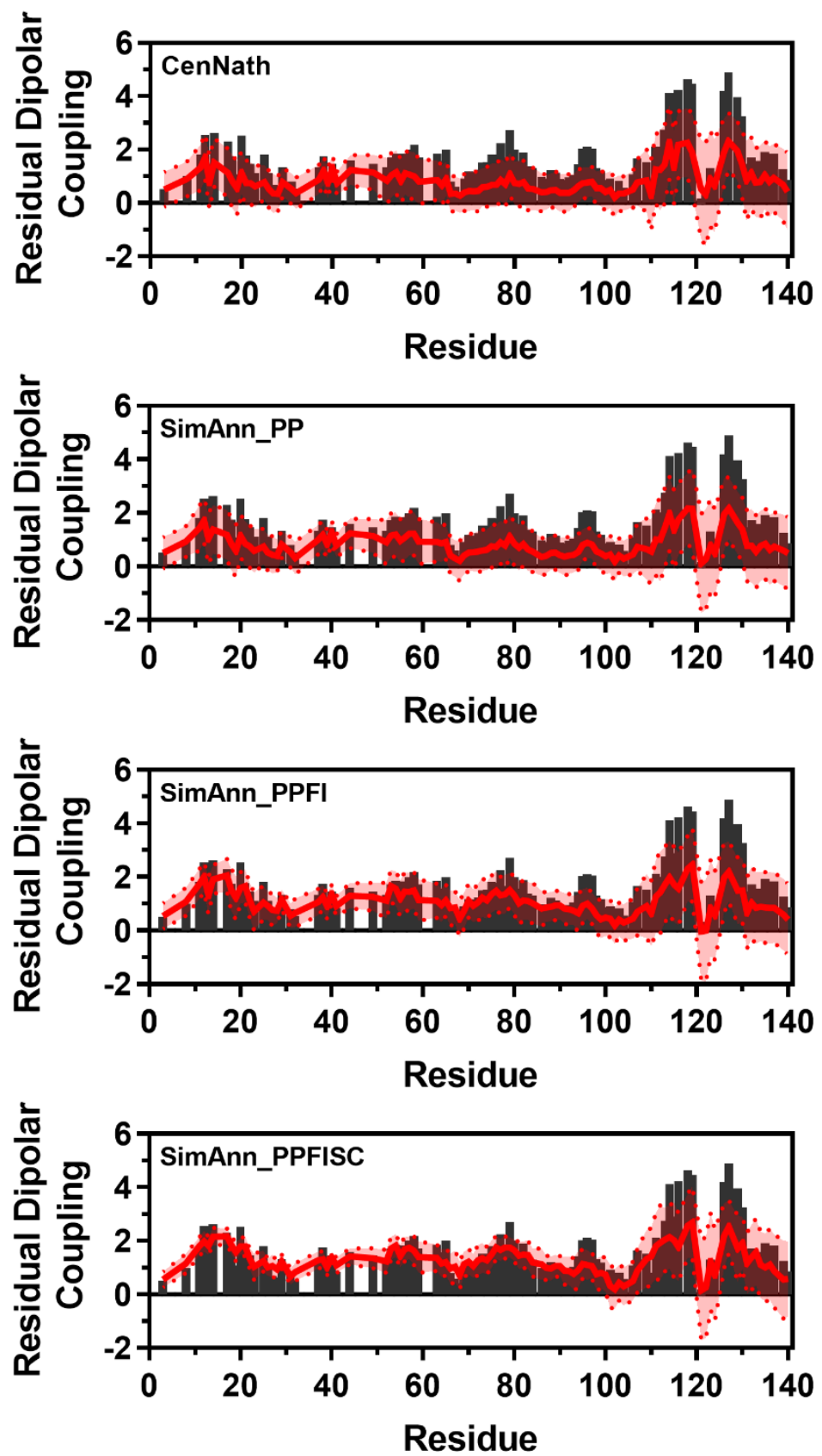

Figure S69: Comparison of Simulated and Experimental Residual Dipolar Coupling Data. Simulated RDC values (red line) from SimAnn_PP (Top), SimAnn_PPFI (Upper Middle), SimAnn_PPFISC (Lower Middle), and SimAnn_PPSC (Bottom) overlayed on experimental data (black bars) from Bertoncini et al. ${ }^{25}$. 

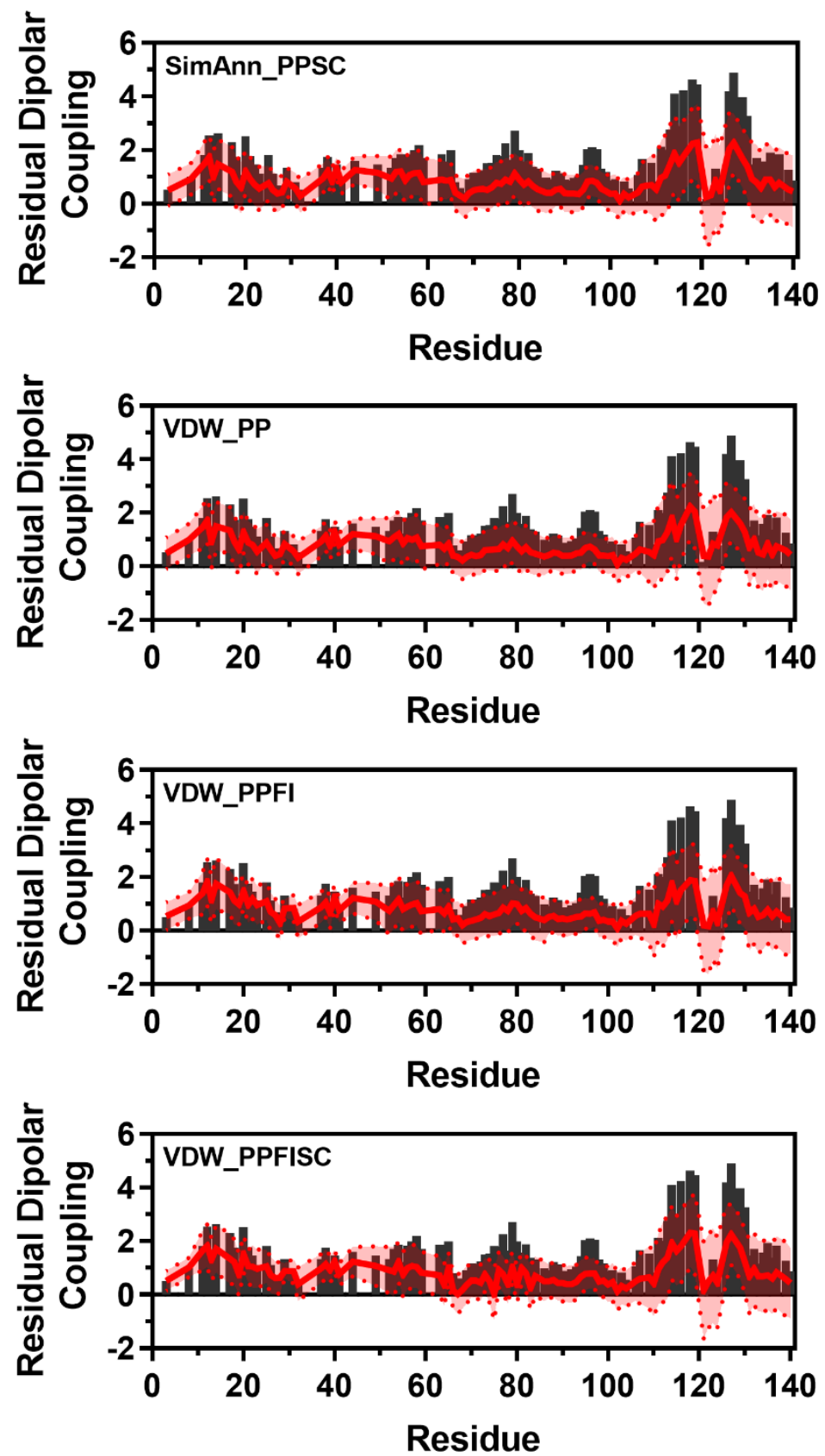

Figure S70: Comparison of Simulated and Experimental Residual Dipolar Coupling Data. Simulated RDC values (red line) from CenNath (Top), VDW_PP (Upper Middle), VDW_PPFI (Lower Middle), and VDW_PPFISC (Bottom) overlayed on experimental data (black bars) from Bertoncini et al. ${ }^{25}$. 

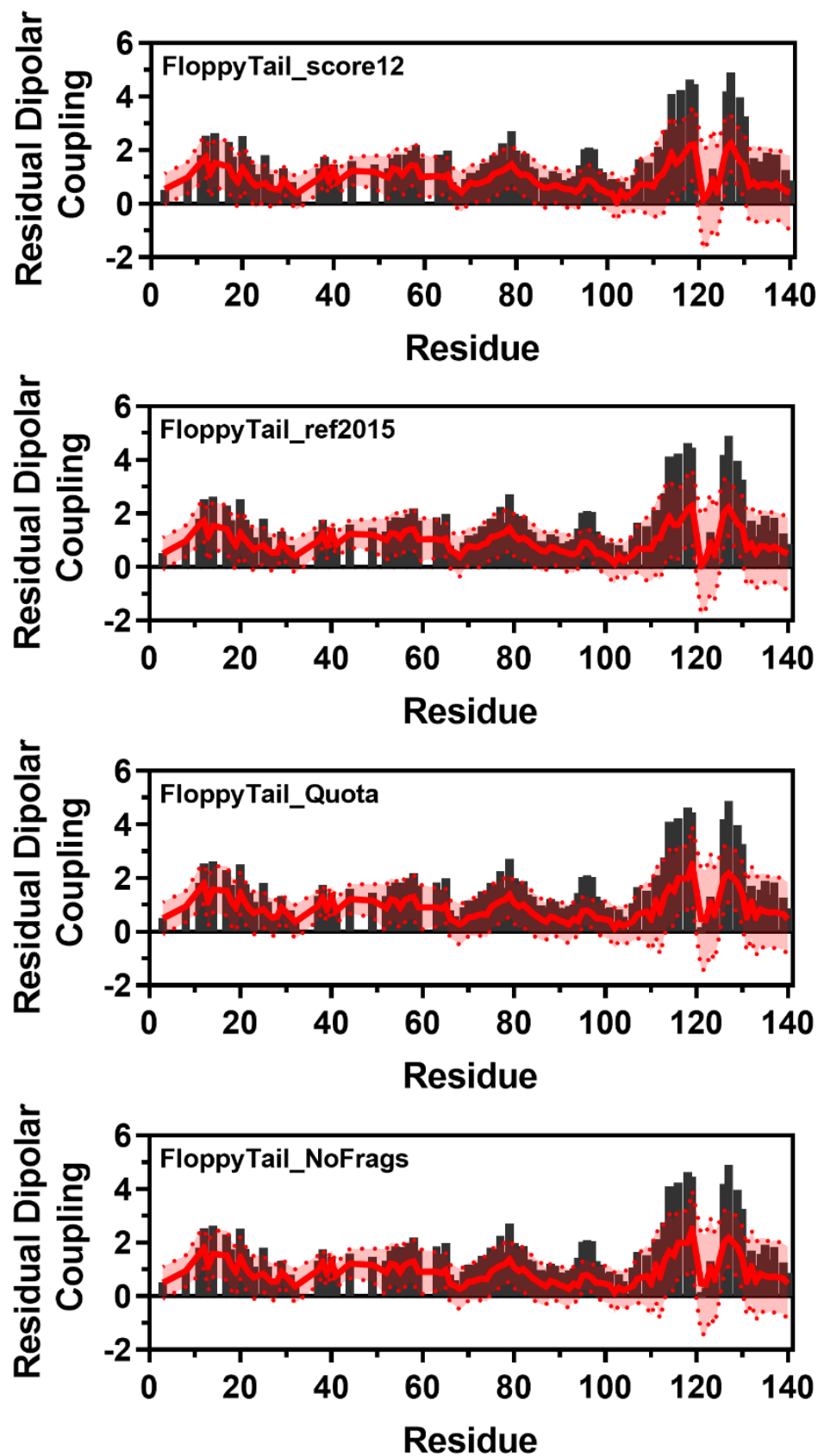

Figure S71: Comparison of Simulated and Experimental Residual Dipolar Coupling Data. Simulated RDC values (red line) from FloppyTail_score12 (Top), FloppyTail_ref2015 (Upper Middle), FloppyTail_Quota (Lower Middle), and FloppyTail_NoFrags (Bottom) overlayed on experimental data (black bars) from Bertoncini et al. ${ }^{25}$. 

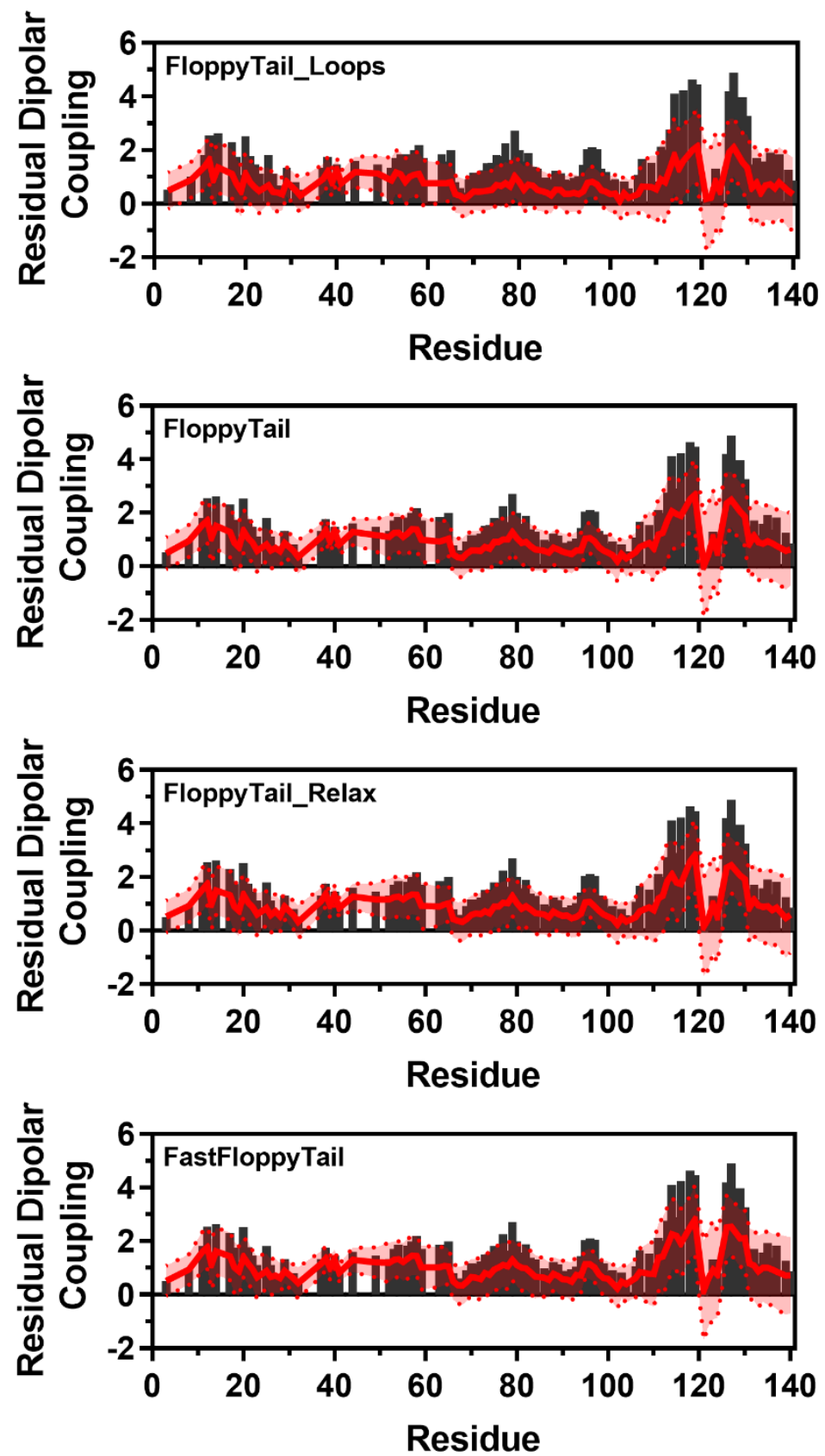

Figure S72: Comparison of Simulated and Experimental Residual Dipolar Coupling Data. Simulated RDC values (red line) from FloppyTail_Loops (Top), FloppyTail (Upper Middle), FloppyTail_Relax (Lower Middle), and FastFloppyTail (Bottom) overlayed on experimental data (black bars) from Bertoncini et al. ${ }^{25}$. 

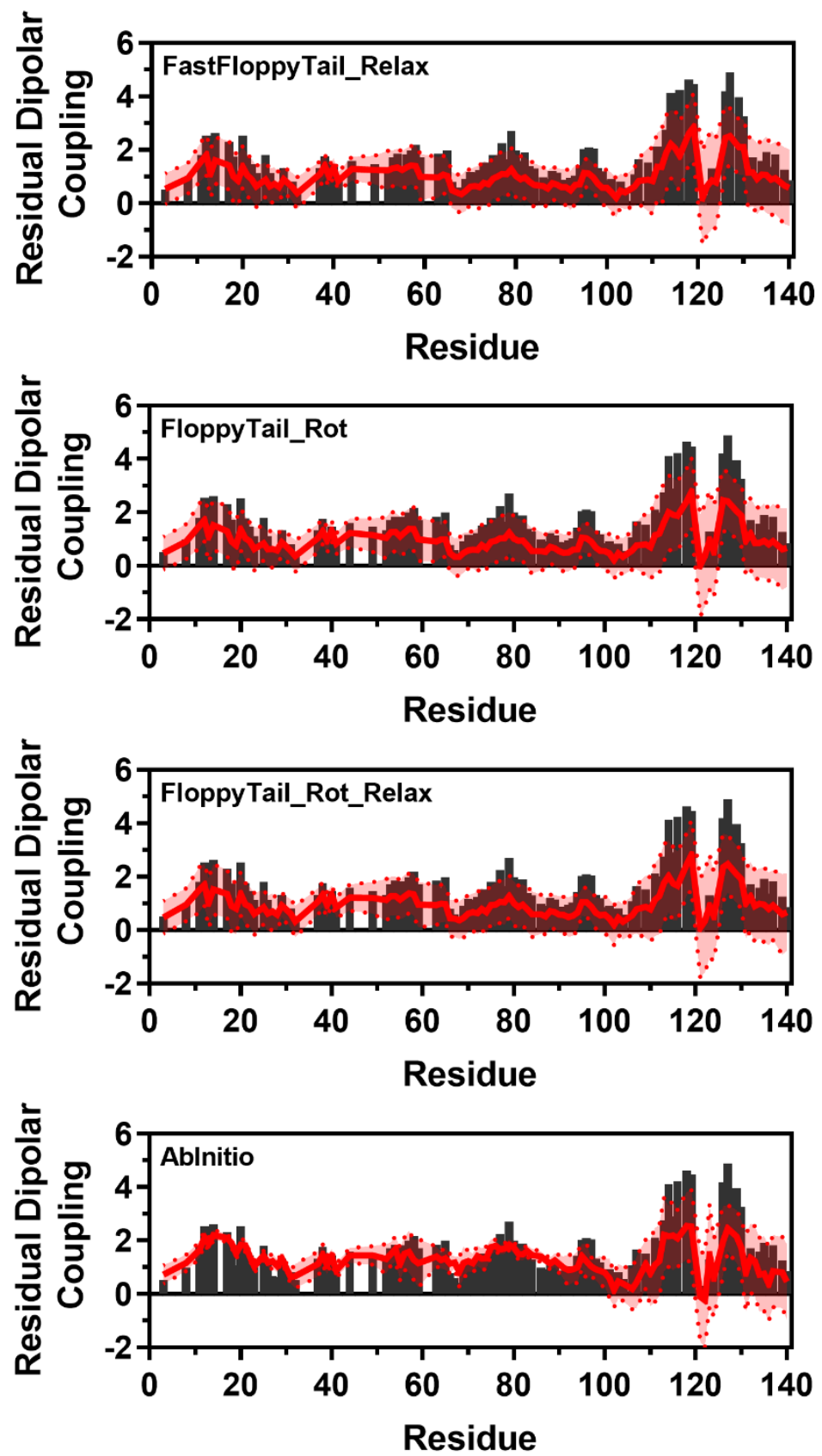

Figure S73: Comparison of Simulated and Experimental Residual Dipolar Coupling Data. Simulated RDC values (red line) from FastFloppyTail_Relax (Top), FloppyTail_Rot (Upper Middle), FloppyTail_Rot_Relax (Lower Middle), and AbInitio (Bottom) overlayed on experimental data (black bars) from Bertoncini et al. ${ }^{25}$. 


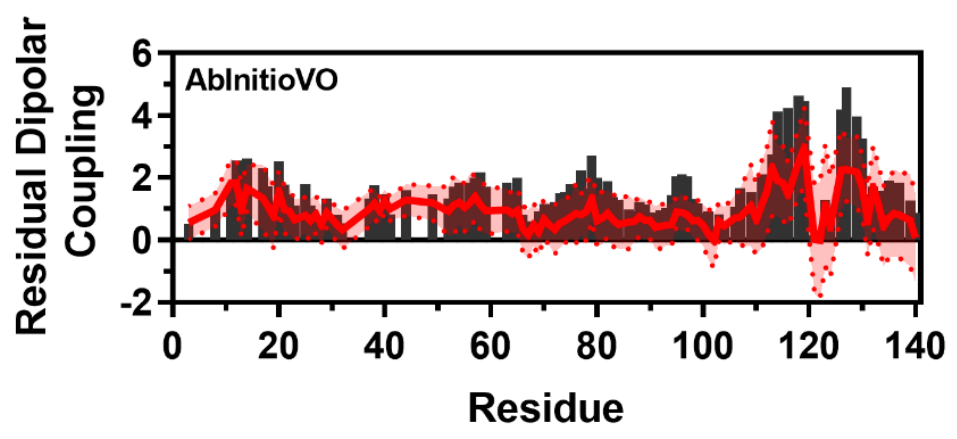

Figure S74: Comparison of Simulated and Experimental Residual Dipolar Coupling Data. Simulated RDC values (red line) from AbInitioVO (Top) overlayed on experimental data (black bars) from Bertoncini et al. ${ }^{25}$. 


\section{Comparison with $\alpha \mathrm{S}$ J-Coupling Data}

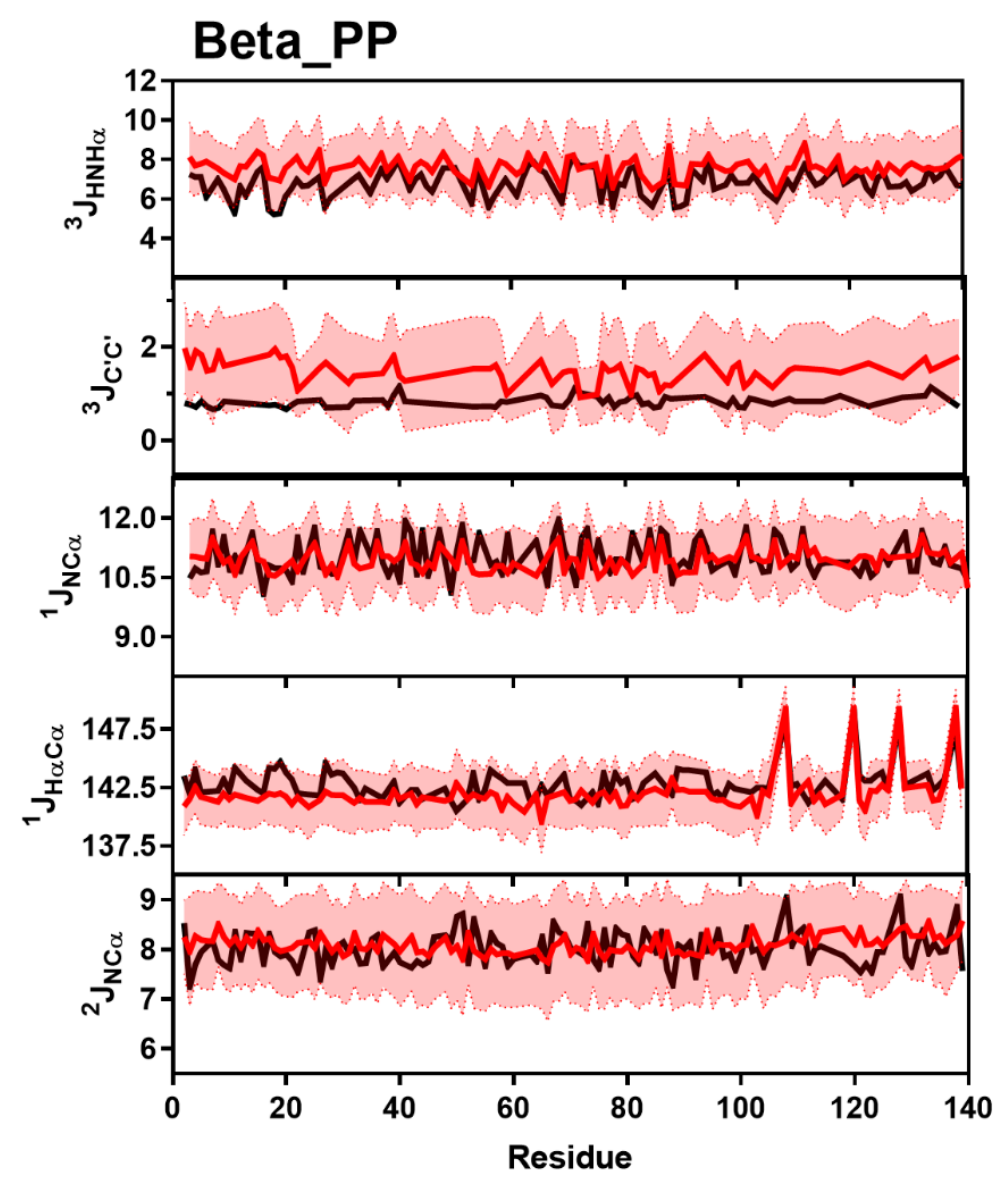

Figure S75: Comparison of Simulated and Experimental J-Coupling Data. J-Coupling values from the Beta_PP ensemble (red) overlayed on experimental data (black) of ${ }^{3} \mathbf{J}_{\mathrm{HNH} \alpha}$ (Top), ${ }^{3} \mathrm{~J}_{\mathrm{CC}}$ (Upper Middle), ${ }^{1} \mathrm{~J}_{\mathrm{H} \alpha \mathrm{C} \alpha}$ (Middle), ${ }^{1} \mathrm{~J}_{\mathrm{NC} \alpha}$ (Lower Middle) and ${ }^{2} \mathrm{~J}_{\mathrm{NC} \alpha}$ (Bottom) from Mantsyzov et al. and Lee et al. ${ }^{13,18}$. 


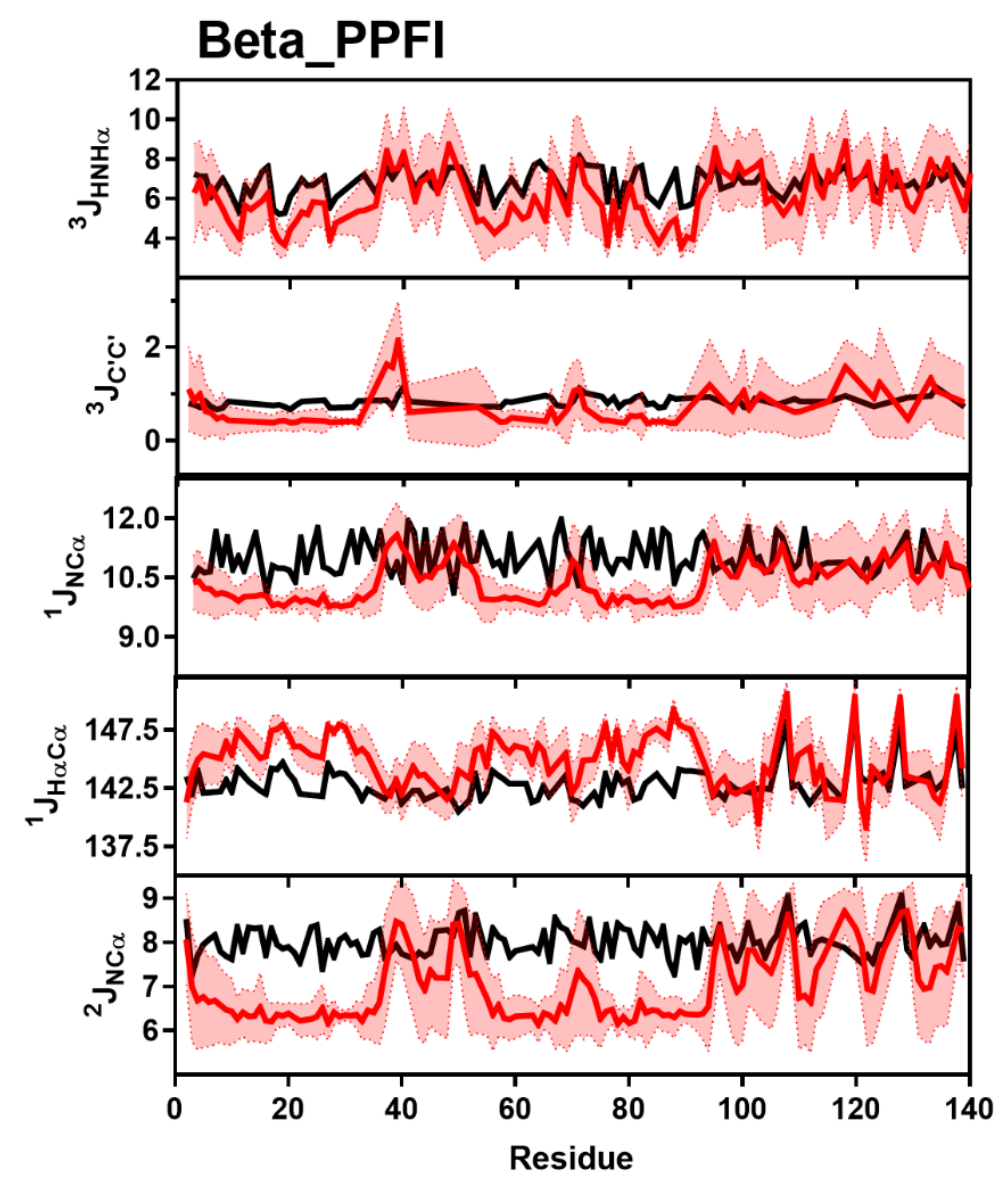

Figure S76: Comparison of Simulated and Experimental J-Coupling Data. Simulated J-Coupling values from the Beta_PPFI ensemble (red) overlayed on experimental data (black) of ${ }^{3} \mathrm{~J}_{\mathrm{HNH} \alpha}$ (Top), ${ }^{3} \mathrm{~J}_{\mathrm{CC}}$ (Upper Middle), ${ }^{1} \mathrm{~J}_{\mathrm{H} \alpha \mathrm{C} \alpha}$ (Middle), ${ }^{1} \mathrm{~J}_{\mathrm{NC} \alpha}$ (Lower Middle) and ${ }^{2} \mathrm{~J}_{\mathrm{NC} \alpha}$ (Bottom) from Mantsyzov et al. and Lee et al. ${ }^{13,18}$. 


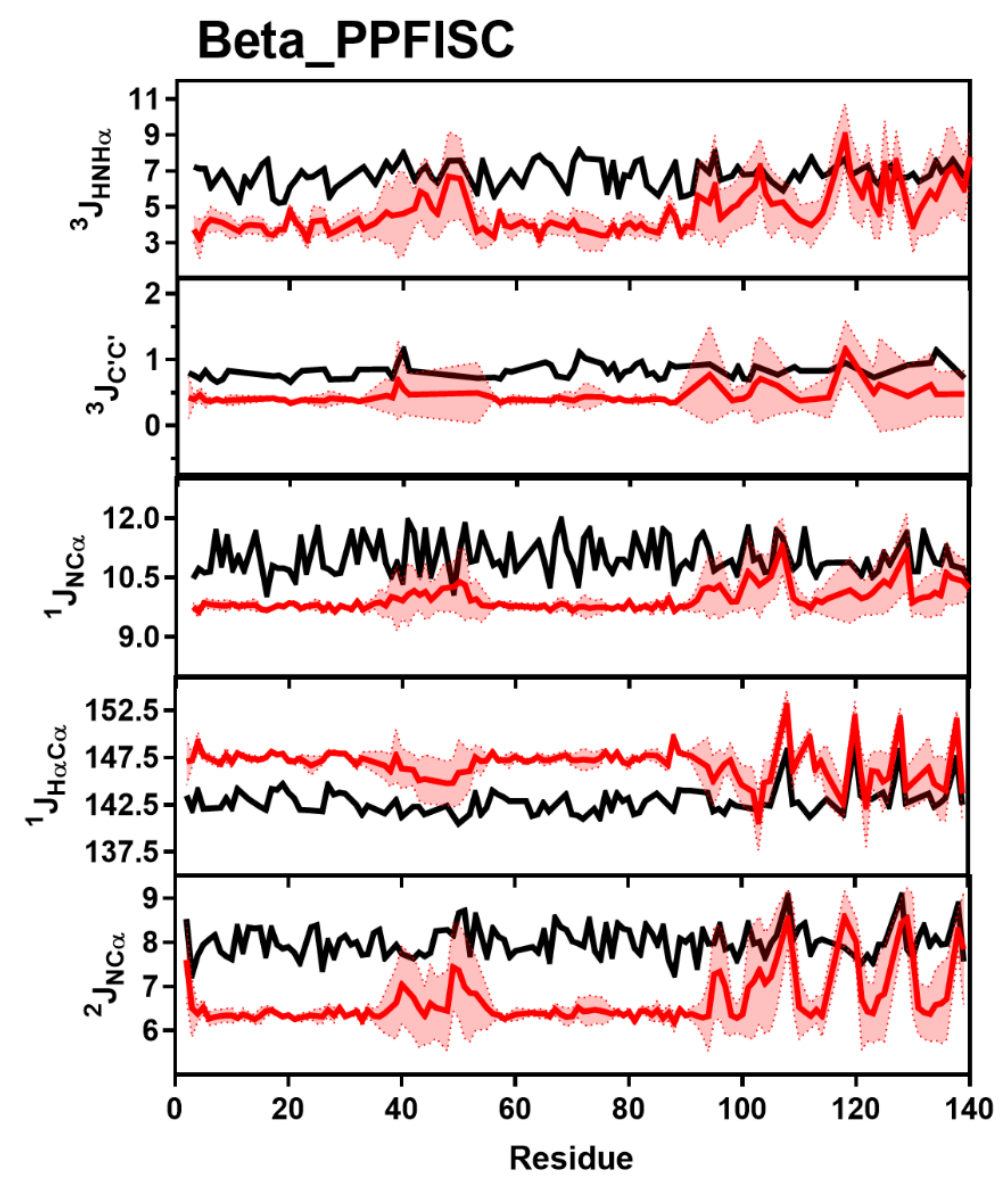

Figure S77: Comparison of Simulated and Experimental J-Coupling Data. Simulated J-Coupling values from the Beta_PPFISC ensemble (red) overlayed on experimental data (black) of ${ }^{3} \mathrm{~J}_{\mathrm{HNH} \alpha}$ (Top), ${ }^{3} \mathrm{~J}_{\mathrm{CC}}$ (Upper Middle), ${ }^{1} \mathrm{~J}_{\mathrm{H} \alpha \mathrm{C} \alpha}$ (Middle), ${ }^{1} \mathrm{~J}_{\mathrm{NC} \alpha}$ (Lower Middle) and ${ }^{2} \mathrm{~J}_{\mathrm{NC} \alpha}$ (Bottom) from Mantsyzov et al. and Lee et al. ${ }^{13,18}$. 


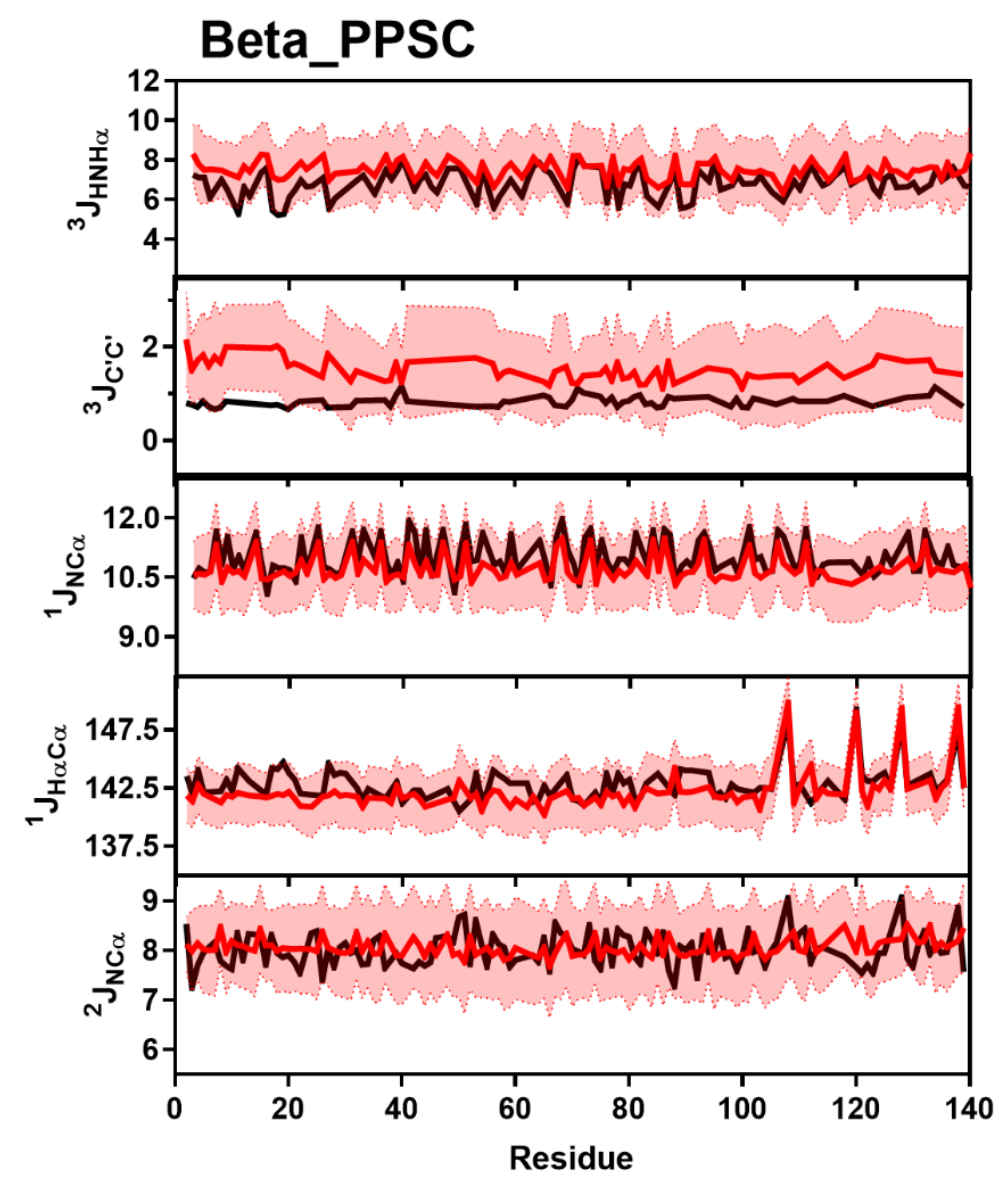

Figure S78: Comparison of Simulated and Experimental J-Coupling Data. Simulated J-Coupling values from the Beta_PPSC ensemble (red) overlayed on experimental data (black) of ${ }^{3} \mathrm{~J}_{\mathrm{HNH} \alpha}$ (Top), ${ }^{3} \mathrm{~J}_{\mathrm{CC}}$ (Upper Middle), ${ }^{1} \mathrm{~J}_{\mathrm{H} \alpha \mathrm{C} \alpha}$ (Middle), ${ }^{1} \mathrm{~J}_{\mathrm{NC} \alpha}$ (Lower Middle) and ${ }^{2} \mathrm{~J}_{\mathrm{NC} \alpha}$ (Bottom) from Mantsyzov et al. and Lee et al. ${ }^{13,18}$. 


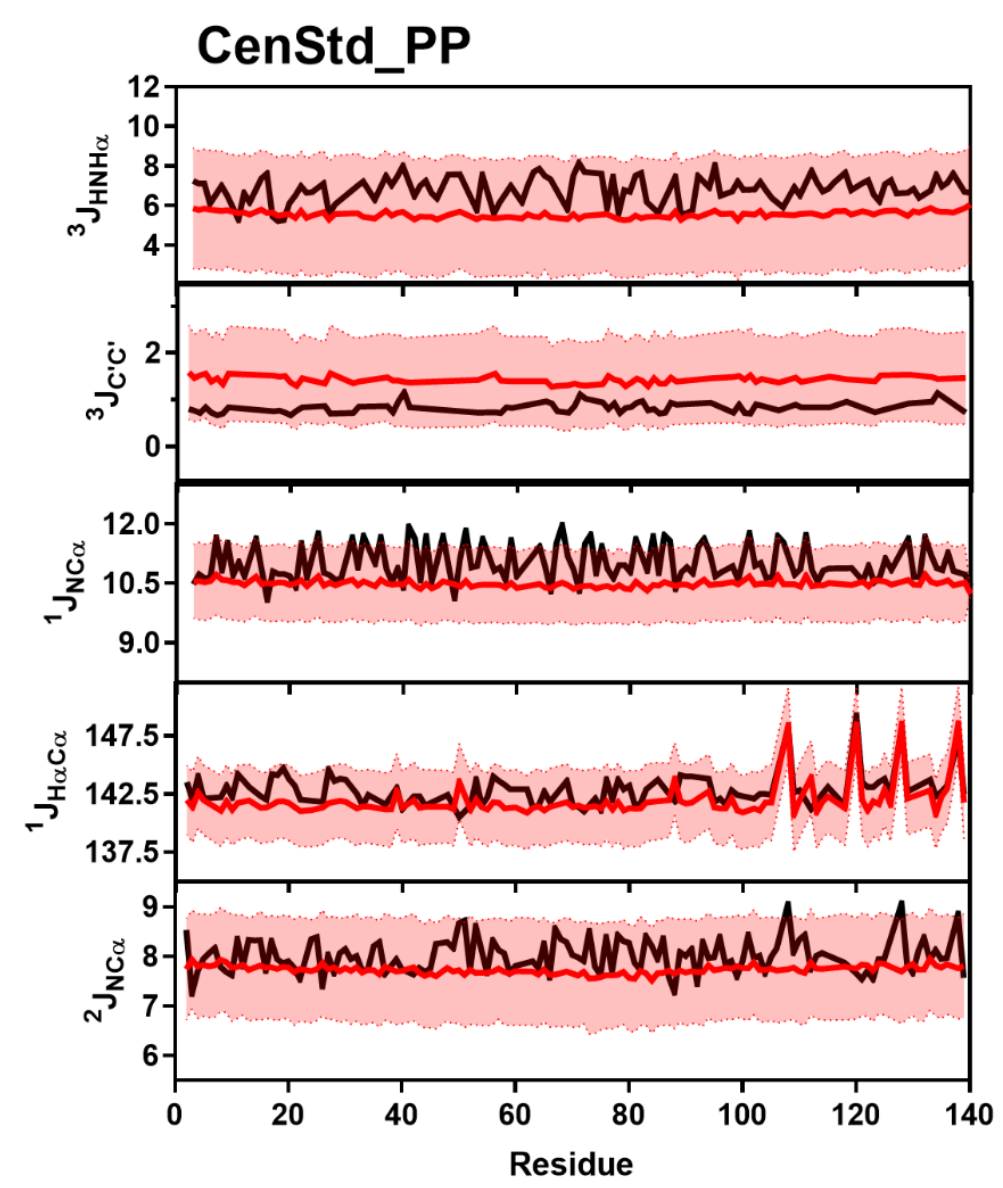

Figure S79: Comparison of Simulated and Experimental J-Coupling Data. Simulated J-Coupling values from the CenStd_PP ensemble (red) overlayed on experimental data (black) of ${ }^{3} \mathrm{~J}_{\mathrm{HNH} \alpha}$ (Top), ${ }^{3} \mathrm{~J}_{\mathrm{CC}}$ (Upper Middle), ${ }^{1} \mathrm{~J}_{\mathrm{H} \alpha \mathrm{C} \alpha}$ (Middle), ${ }^{1} \mathrm{~J}_{\mathrm{NC} \alpha}$ (Lower Middle) and ${ }^{2} \mathrm{~J}_{\mathrm{NC} \alpha}$ (Bottom) from Mantsyzov et al. and Lee et al. ${ }^{13,18}$. 


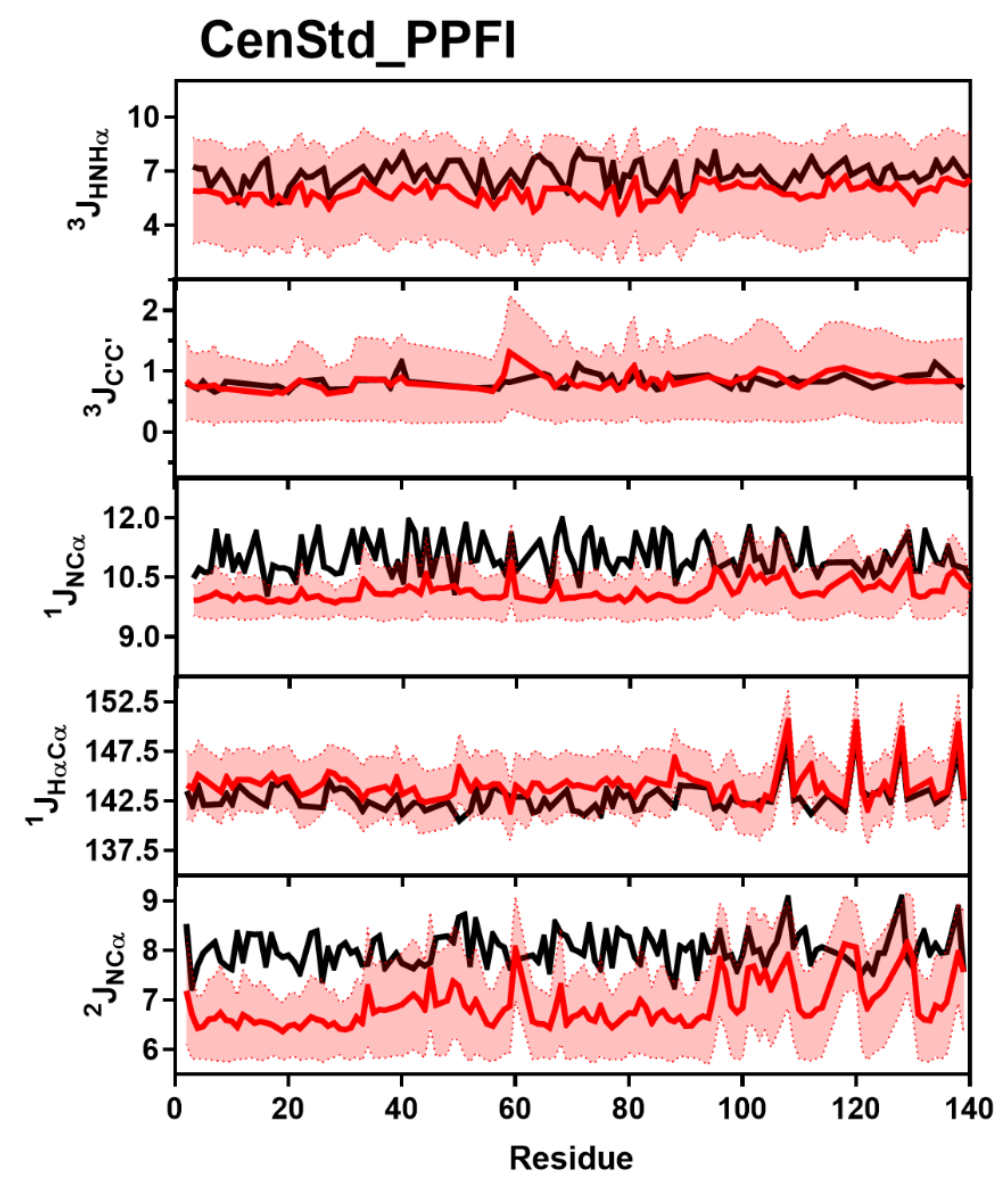

Figure S80: Comparison of Simulated and Experimental J-Coupling Data. Simulated J-Coupling values from the CenStd_PPFI ensemble (red) overlayed on experimental data (black) of ${ }^{3} \mathrm{~J}_{\mathrm{HNH} \alpha}$ (Top), ${ }^{3} \mathrm{~J}_{\mathrm{CC}}$ (Upper Middle), ${ }^{1} \mathrm{~J}_{\mathrm{H} \alpha \mathrm{C} \alpha}$ (Middle), ${ }^{1} \mathrm{~J}_{\mathrm{NC} \alpha}$ (Lower Middle) and ${ }^{2} \mathrm{~J}_{\mathrm{NC} \alpha}$ (Bottom) from Mantsyzov et al. and Lee et al. ${ }^{13,18}$. 


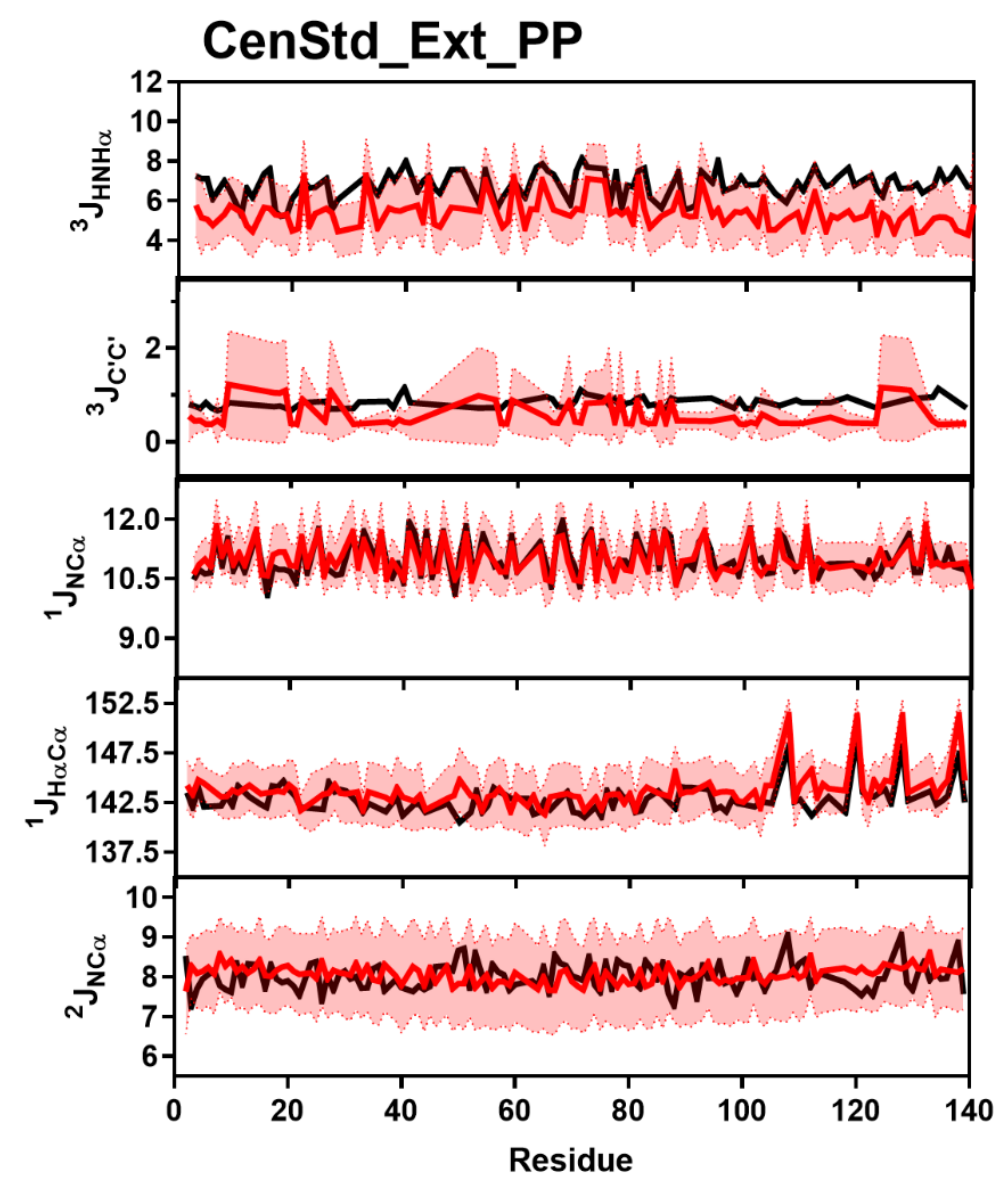

Figure S81: Comparison of Simulated and Experimental J-Coupling Data. Simulated J-Coupling values from the CenStd_Ext_PP ensemble (red) overlayed on experimental data (black) of ${ }^{3} \mathrm{~J}_{\mathrm{HNH} \alpha}$ (Top), ${ }^{3} \mathrm{~J}_{\mathrm{CC}}$ (Upper Middle), ${ }^{1} \mathrm{~J}_{\mathrm{H} \alpha \mathrm{C} \alpha}$ (Middle), ${ }^{1} \mathrm{~J}_{\mathrm{NC} \alpha}$ (Lower Middle) and ${ }^{2} \mathrm{~J}_{\mathrm{NC} \alpha}$ (Bottom) from Mantsyzov et al. and Lee et al. ${ }^{13,18}$. 


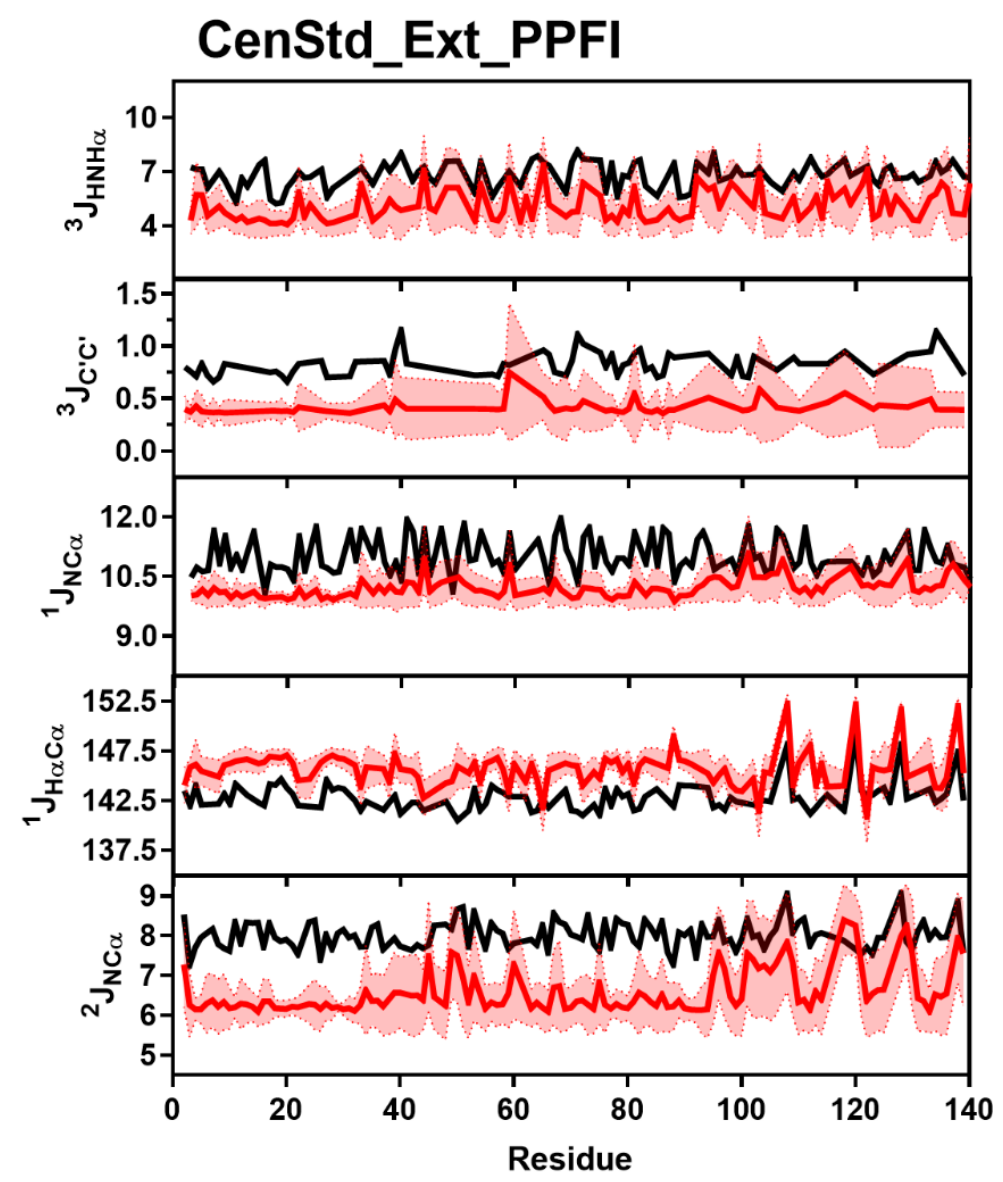

Figure S82: Comparison of Simulated and Experimental J-Coupling Data. Simulated J-Coupling values from the CenStd_Ext_PPFI ensemble (red) overlayed on experimental data (black) of ${ }^{3} \mathrm{~J}_{\mathrm{HNH} \alpha}$ (Top), ${ }^{3} \mathrm{~J}_{\mathrm{CC}}$ (Upper Middle), ${ }^{1} \mathrm{~J}_{\mathrm{H} \alpha \mathrm{C} \alpha}$ (Middle), ${ }^{1} \mathrm{~J}_{\mathrm{NC} \alpha}$ (Lower Middle) and ${ }^{2} \mathrm{~J}_{\mathrm{NC} \alpha}$ (Bottom) from Mantsyzov et al. and Lee et al. ${ }^{13,18}$. 


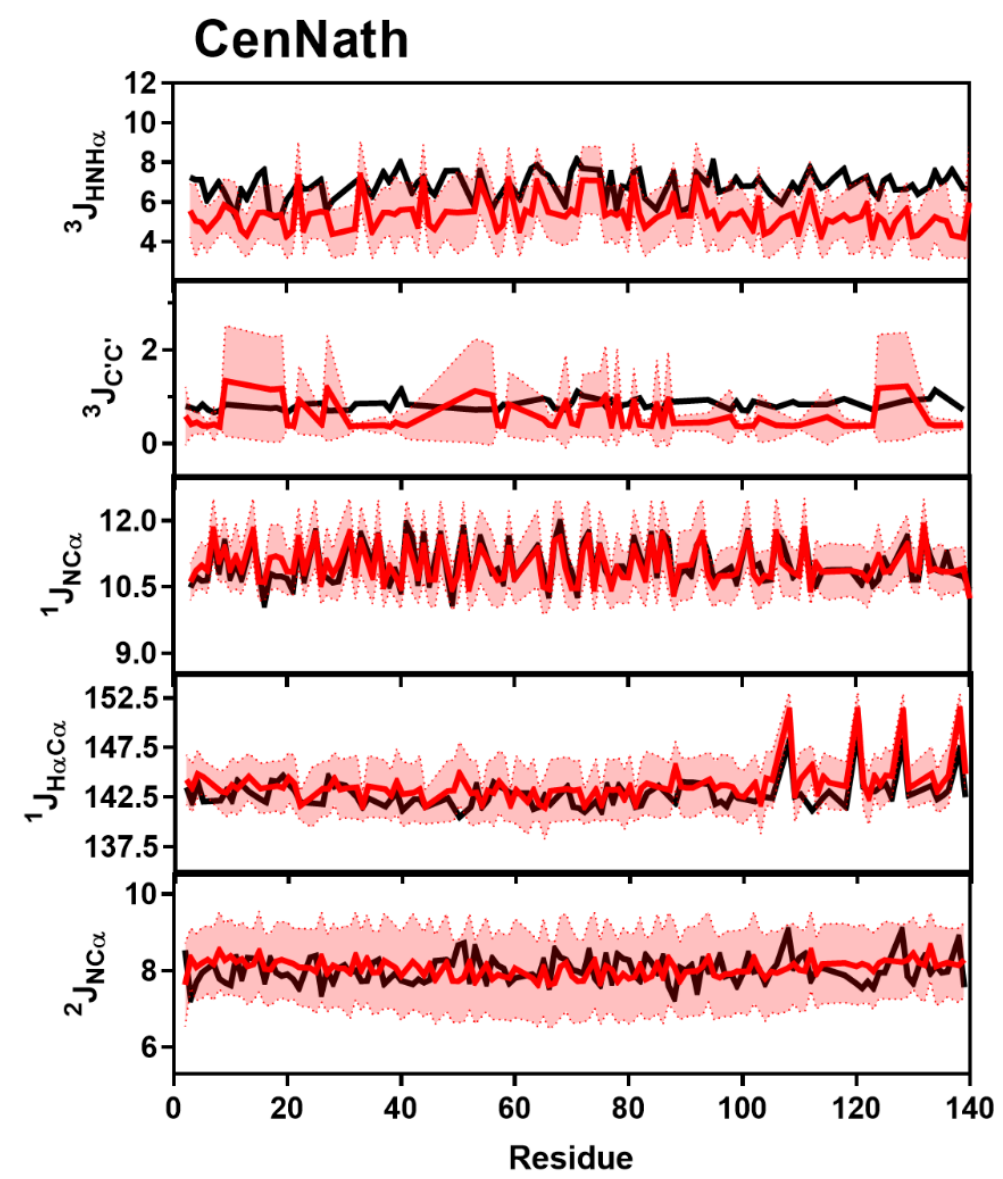

Figure S83: Comparison of Simulated and Experimental J-Coupling Data. Simulated J-Coupling values from the CenNath ensemble (red) overlayed on experimental data (black) of ${ }^{3} \mathrm{~J}_{\mathrm{HNH} \alpha}$ (Top), ${ }^{3} \mathrm{~J}_{\mathrm{CC}}$ (Upper Middle), ${ }^{1} \mathrm{~J}_{\mathrm{H} \alpha \mathrm{C} \alpha}$ (Middle), ${ }^{1} \mathrm{~J}_{\mathrm{NC} \alpha}$ (Lower Middle) and ${ }^{2} \mathrm{~J}_{\mathrm{NC} \alpha}$ (Bottom) from Mantsyzov et al. and Lee et al. ${ }^{13,18}$. 


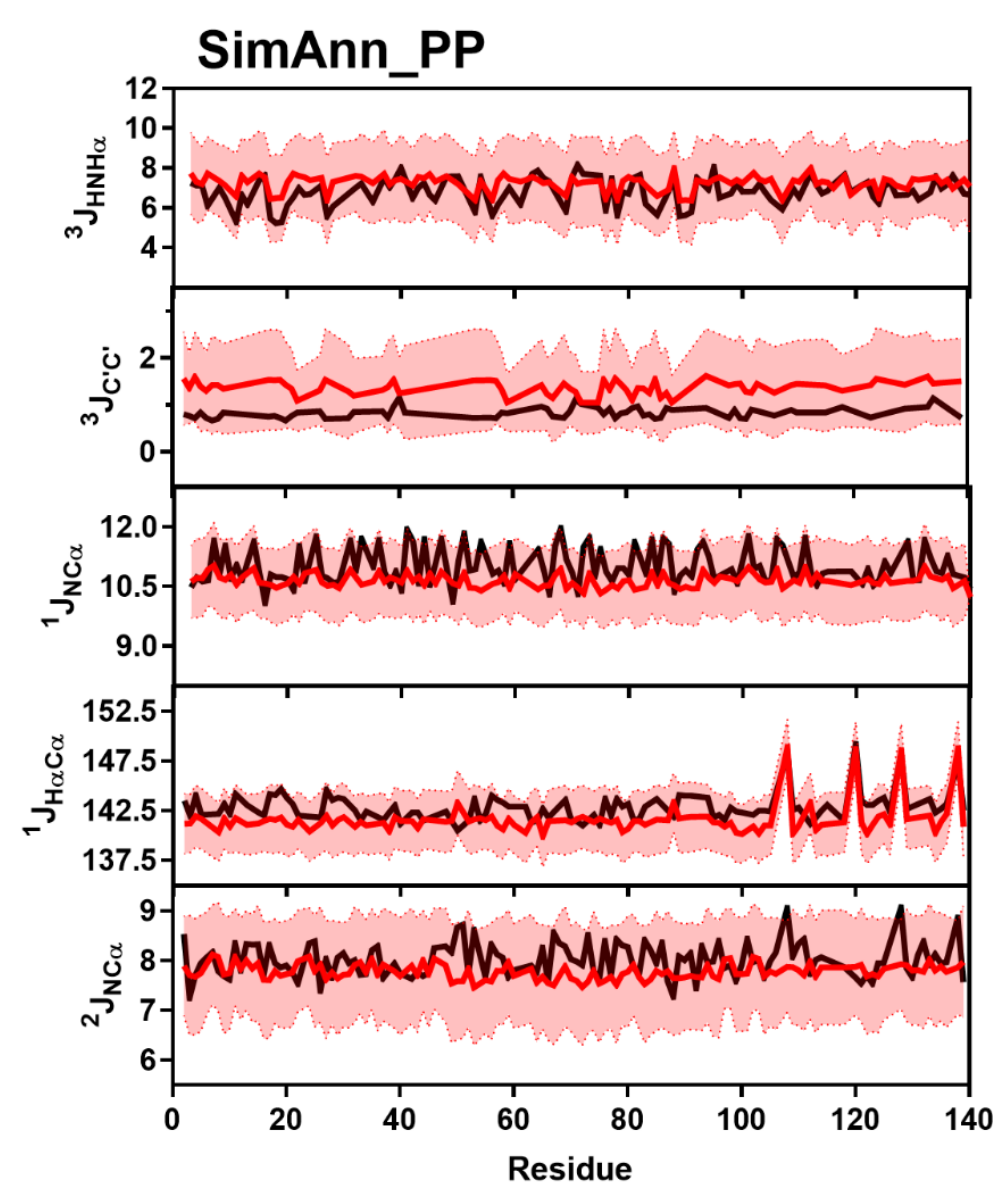

Figure S84: Comparison of Simulated and Experimental J-Coupling Data. Simulated J-Coupling values from the SimAnn_PP ensemble (red) overlayed on experimental data (black) of ${ }^{3} \mathrm{~J}_{\mathrm{HNH} \alpha}$ (Top), ${ }^{3} \mathrm{~J}_{\mathrm{CC}}$ (Upper Middle), ${ }^{1} \mathrm{~J}_{\mathrm{H} \alpha \mathrm{C} \alpha}$ (Middle), ${ }^{1} \mathrm{~J}_{\mathrm{NC} \alpha}$ (Lower Middle) and ${ }^{2} \mathrm{~J}_{\mathrm{NC} \alpha}$ (Bottom) from Mantsyzov et al. and Lee et al. ${ }^{13,18}$. 


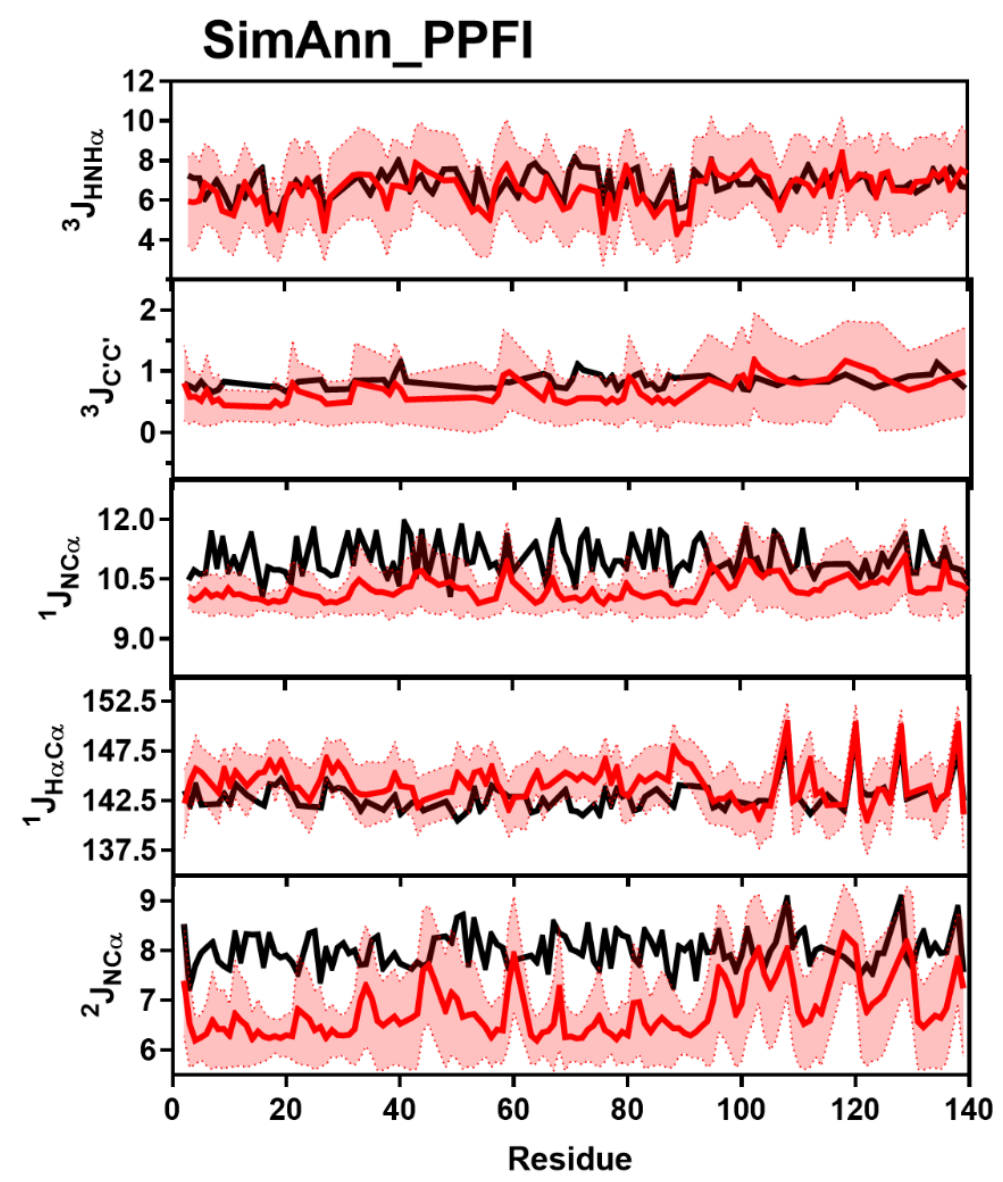

Figure S85: Comparison of Simulated and Experimental J-Coupling Data. Simulated J-Coupling values from the SimAnn_PPFI ensemble (red) overlayed on experimental data (black) of ${ }^{3} \mathrm{~J}_{\mathrm{HNH} \alpha}$ (Top), ${ }^{3} \mathrm{~J}_{\mathrm{CC}}$ (Upper Middle), ${ }^{1} \mathrm{~J}_{\mathrm{H} \alpha \mathrm{C} \alpha}$ (Middle), ${ }^{1} \mathrm{~J}_{\mathrm{NC} \alpha}$ (Lower Middle) and ${ }^{2} \mathrm{~J}_{\mathrm{NC} \alpha}$ (Bottom) from Mantsyzov et al. and Lee et al. ${ }^{13,18}$. 


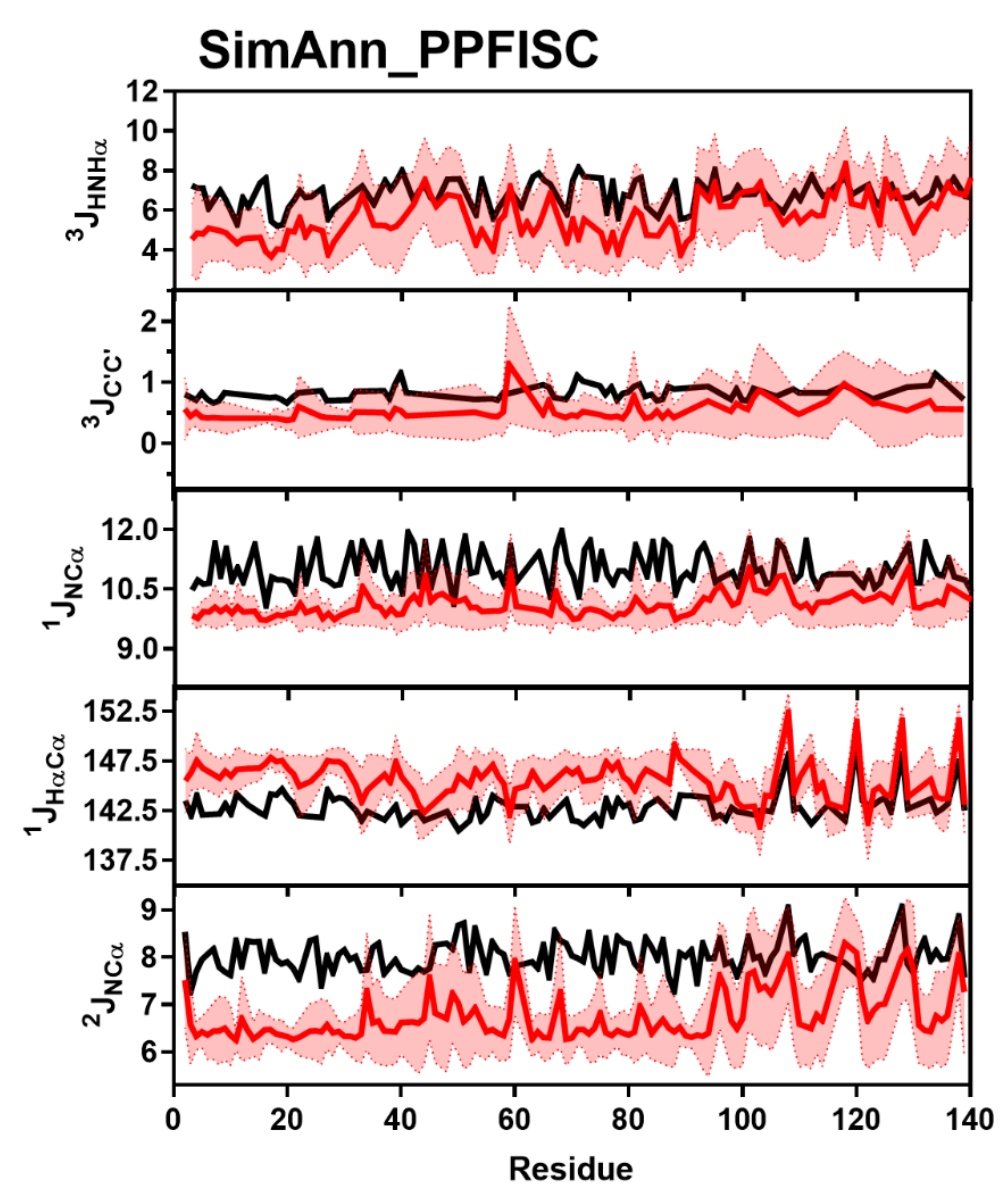

Figure S86: Comparison of Simulated and Experimental J-Coupling Data. Simulated J-Coupling values from the SimAnn_PPFISC ensemble (red) overlayed on experimental data (black) of ${ }^{3} \mathrm{~J}_{\mathrm{HNH} \alpha}$ (Top), ${ }^{3} \mathrm{~J}_{\mathrm{CC}}$ (Upper Middle), ${ }^{1} \mathrm{~J}_{\mathrm{H} \alpha \mathrm{C} \alpha}$ (Middle), ${ }^{1} \mathrm{~J}_{\mathrm{NC} \alpha}$ (Lower Middle) and ${ }^{2} \mathrm{~J}_{\mathrm{NC} \alpha}$ (Bottom) from Mantsyzov et al. and Lee et al. ${ }^{13,18}$. 


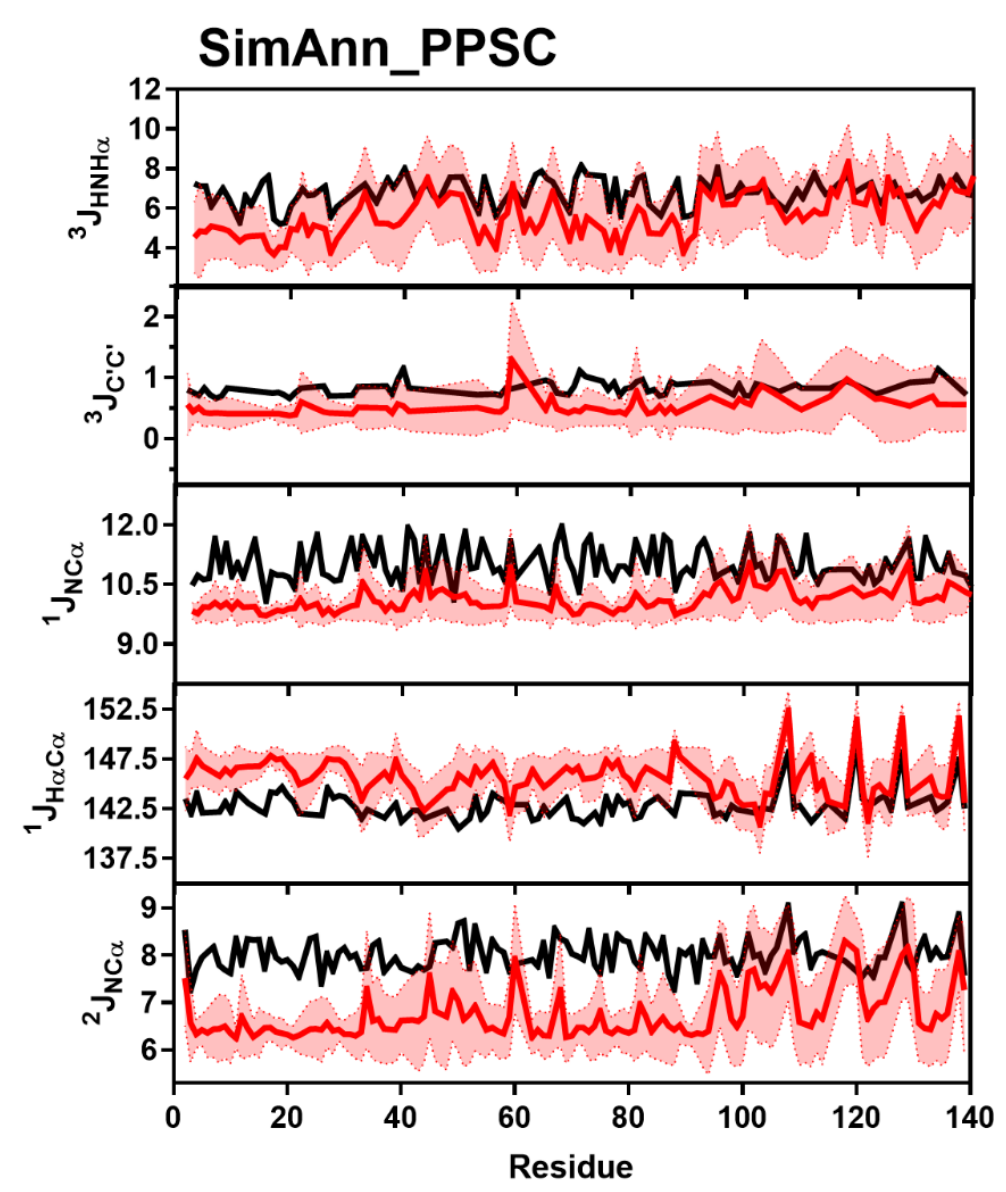

Figure S87: Comparison of Simulated and Experimental J-Coupling Data. Simulated J-Coupling values from the SimAnn_PPSC ensemble (red) overlayed on experimental data (black) of ${ }^{3} \mathrm{~J}_{\mathrm{HNH} \alpha}$ (Top), ${ }^{3} \mathrm{~J}_{\mathrm{CC}}$ (Upper Middle), ${ }^{1} \mathrm{~J}_{\mathrm{H} \alpha \mathrm{C} \alpha}$ (Middle), ${ }^{1} \mathrm{~J}_{\mathrm{NC} \alpha}$ (Lower Middle) and ${ }^{2} \mathrm{~J}_{\mathrm{NC} \alpha}$ (Bottom) from Mantsyzov et al. and Lee et al. ${ }^{13,18}$. 


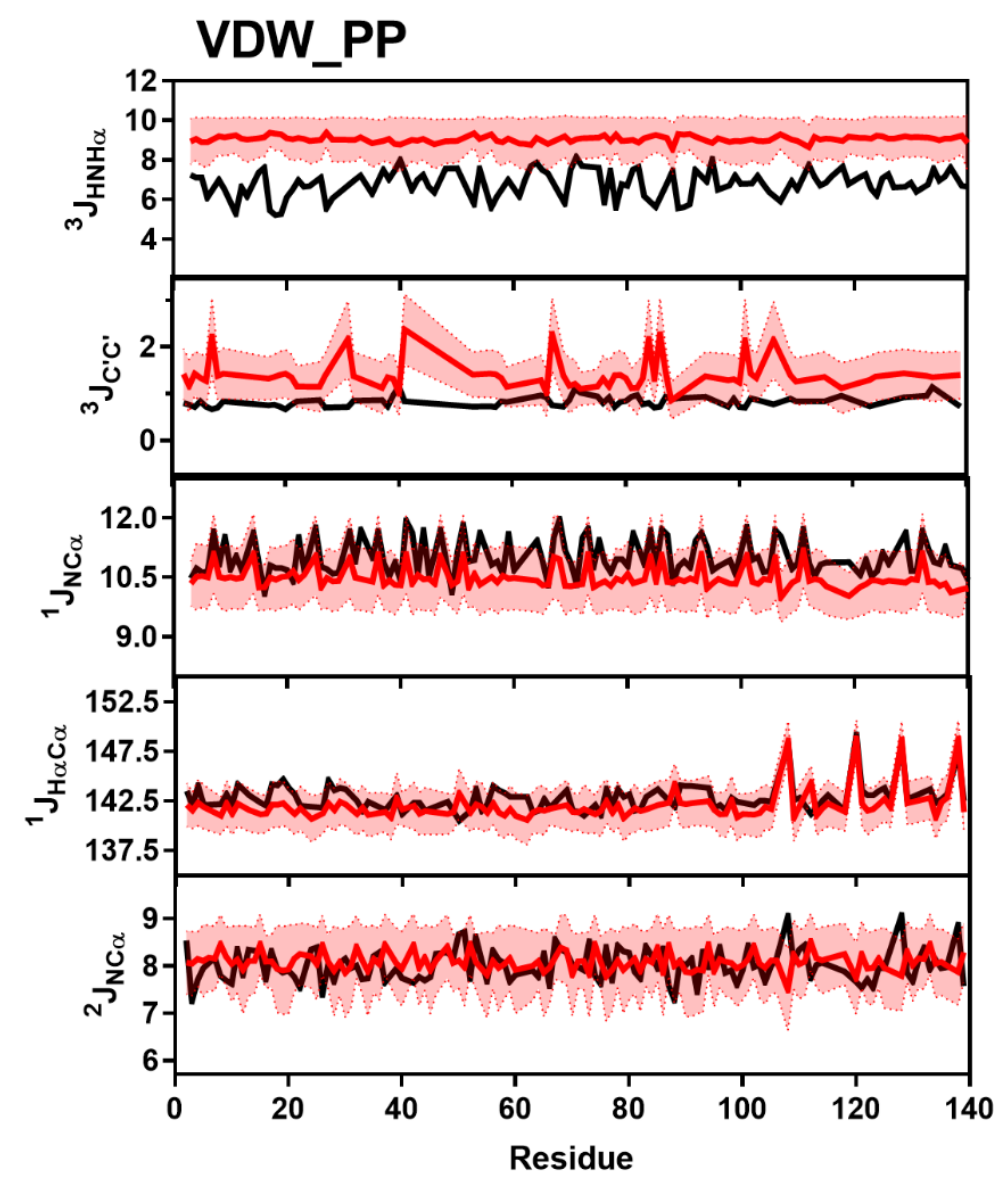

Figure S88: Comparison of Simulated and Experimental J-Coupling Data. Simulated J-Coupling values from the VDW_PP ensemble (red) overlayed on experimental data (black) of ${ }^{3} \mathrm{~J}_{\mathrm{HNH} \alpha}$ (Top), ${ }^{3} \mathrm{~J}_{\mathrm{CC}}$ (Upper Middle), ${ }^{1} \mathrm{~J}_{\mathrm{H} \alpha \mathrm{C} \alpha}$ (Middle), ${ }^{1} \mathrm{~J}_{\mathrm{NC} \alpha}$ (Lower Middle) and ${ }^{2} \mathrm{~J}_{\mathrm{NC} \alpha}$ (Bottom) from Mantsyzov et al. and Lee et al. ${ }^{13,18}$. 


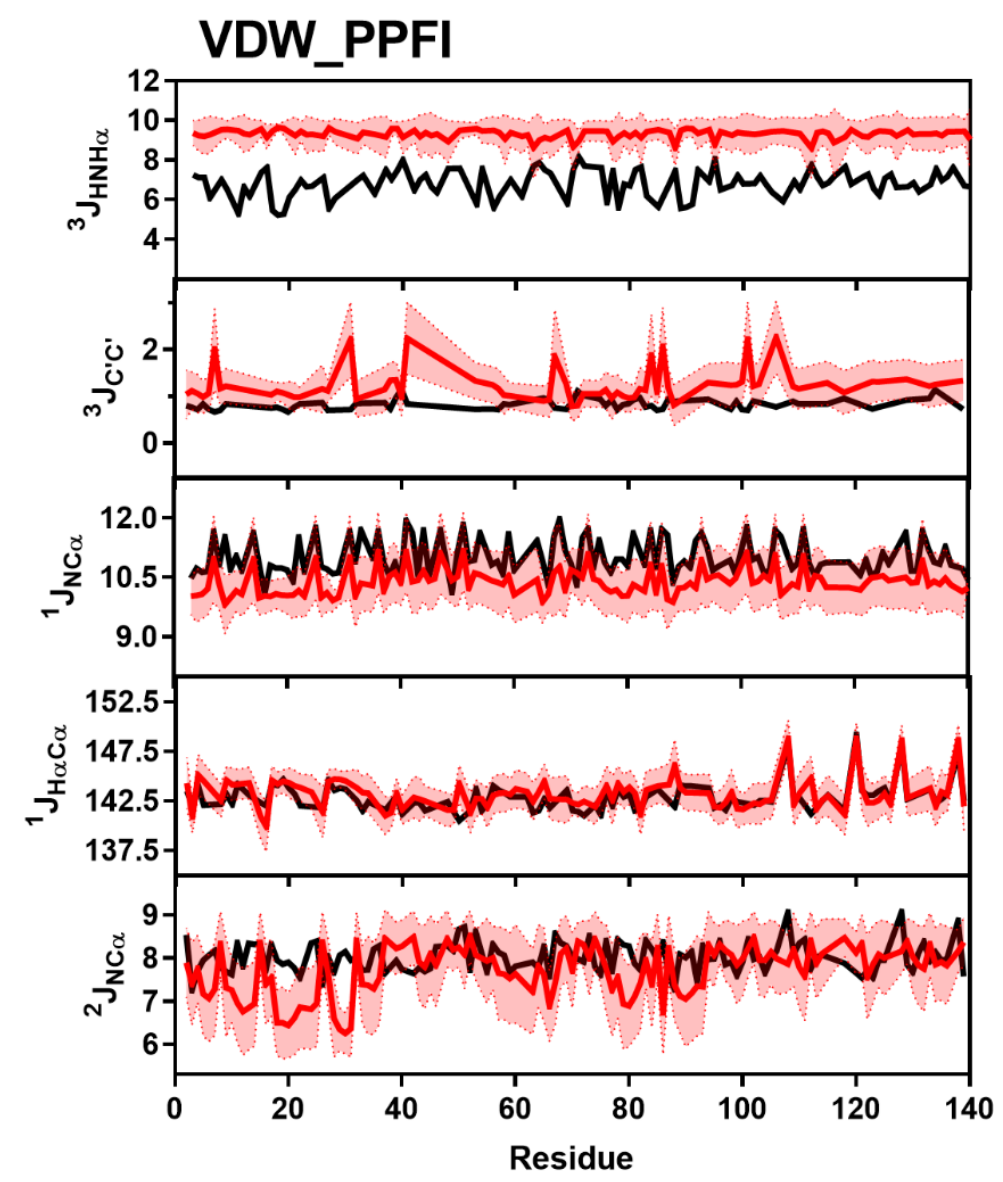

Figure S89: Comparison of Simulated and Experimental J-Coupling Data. Simulated J-Coupling values from the VDW_PPFI ensemble (red) overlayed on experimental data (black) of ${ }^{3} \mathrm{~J}_{\mathrm{HNH} \alpha}$ (Top), ${ }^{3} \mathrm{~J}_{\mathrm{CC}}$ (Upper Middle), ${ }^{1} \mathrm{~J}_{\mathrm{H} \alpha \mathrm{C} \alpha}$ (Middle), ${ }^{1} \mathrm{~J}_{\mathrm{NC} \alpha}$ (Lower Middle) and ${ }^{2} \mathrm{~J}_{\mathrm{NC} \alpha}$ (Bottom) from Mantsyzov et al. and Lee et al. ${ }^{13,18}$. 


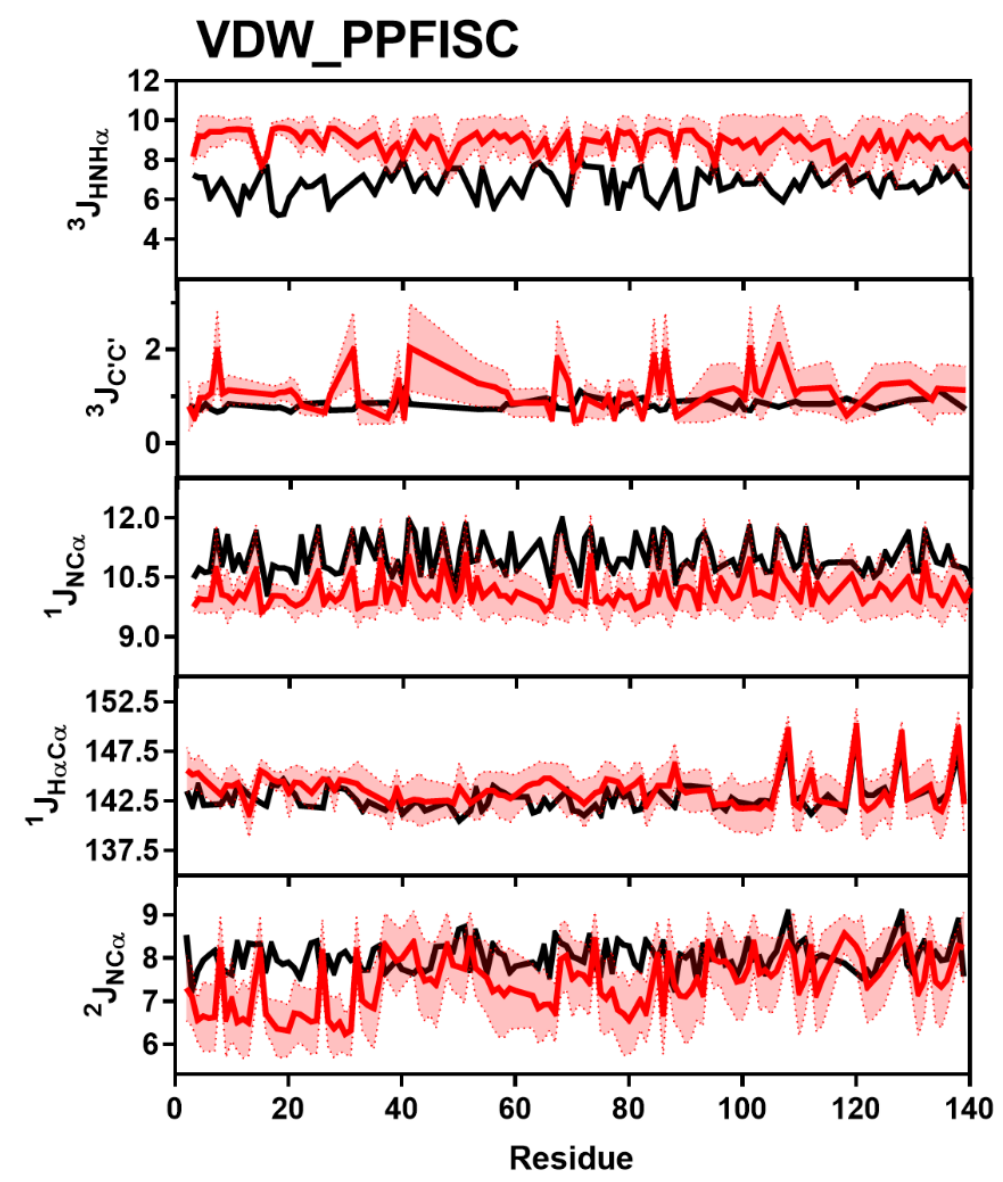

Figure S90: Comparison of Simulated and Experimental J-Coupling Data. Simulated J-Coupling values from the VDW_PPFISC ensemble (red) overlayed on experimental data (black) of ${ }^{3} \mathrm{~J}_{\mathrm{HNH} \alpha}$ (Top), ${ }^{3} \mathrm{~J}_{\mathrm{CC}}$ (Upper Middle), ${ }^{1} \mathrm{~J}_{\mathrm{H} \alpha \mathrm{C} \alpha}$ (Middle), ${ }^{1} \mathrm{~J}_{\mathrm{NC} \alpha}$ (Lower Middle) and ${ }^{2} \mathrm{~J}_{\mathrm{NC} \alpha}$ (Bottom) from Mantsyzov et al. and Lee et al. ${ }^{13,18}$. 


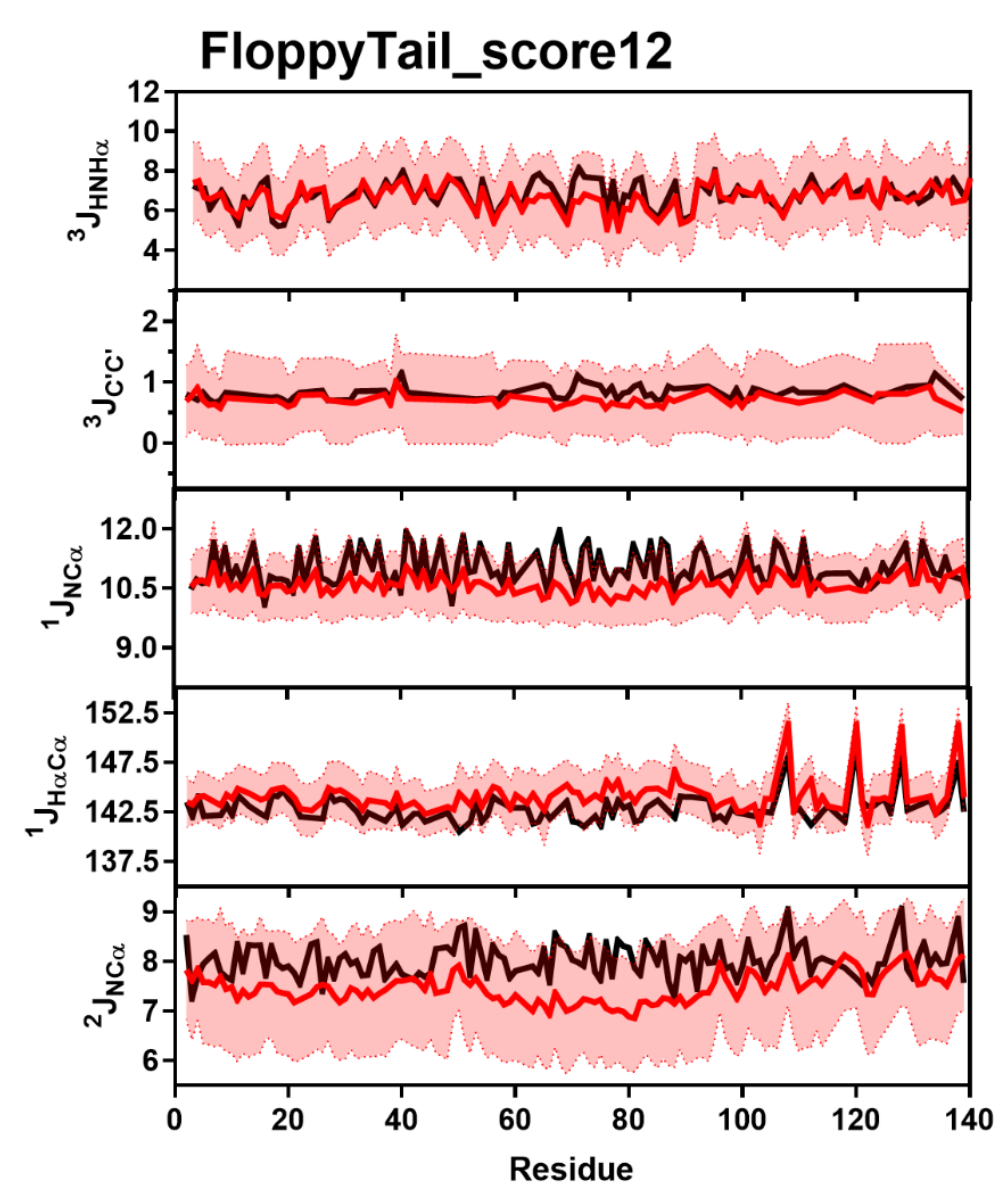

Figure S91: Comparison of Simulated and Experimental J-Coupling Data. Simulated J-Coupling values from the FloppyTail_score12 ensemble (red) overlayed on experimental data (black) of ${ }^{3} \mathrm{~J}_{\mathrm{HNH} \alpha}$ (Top), ${ }^{3} \mathrm{~J}_{\mathrm{CC}}$ (Upper Middle), ${ }^{1} \mathrm{~J}_{\mathrm{H} \alpha \mathrm{C} \alpha}$ (Middle), ${ }^{1} \mathrm{~J}_{\mathrm{NC} \alpha}$ (Lower Middle) and ${ }^{2} \mathrm{~J}_{\mathrm{NC} \alpha}$ (Bottom) from Mantsyzov et al. and Lee et al. ${ }^{13,18}$. 


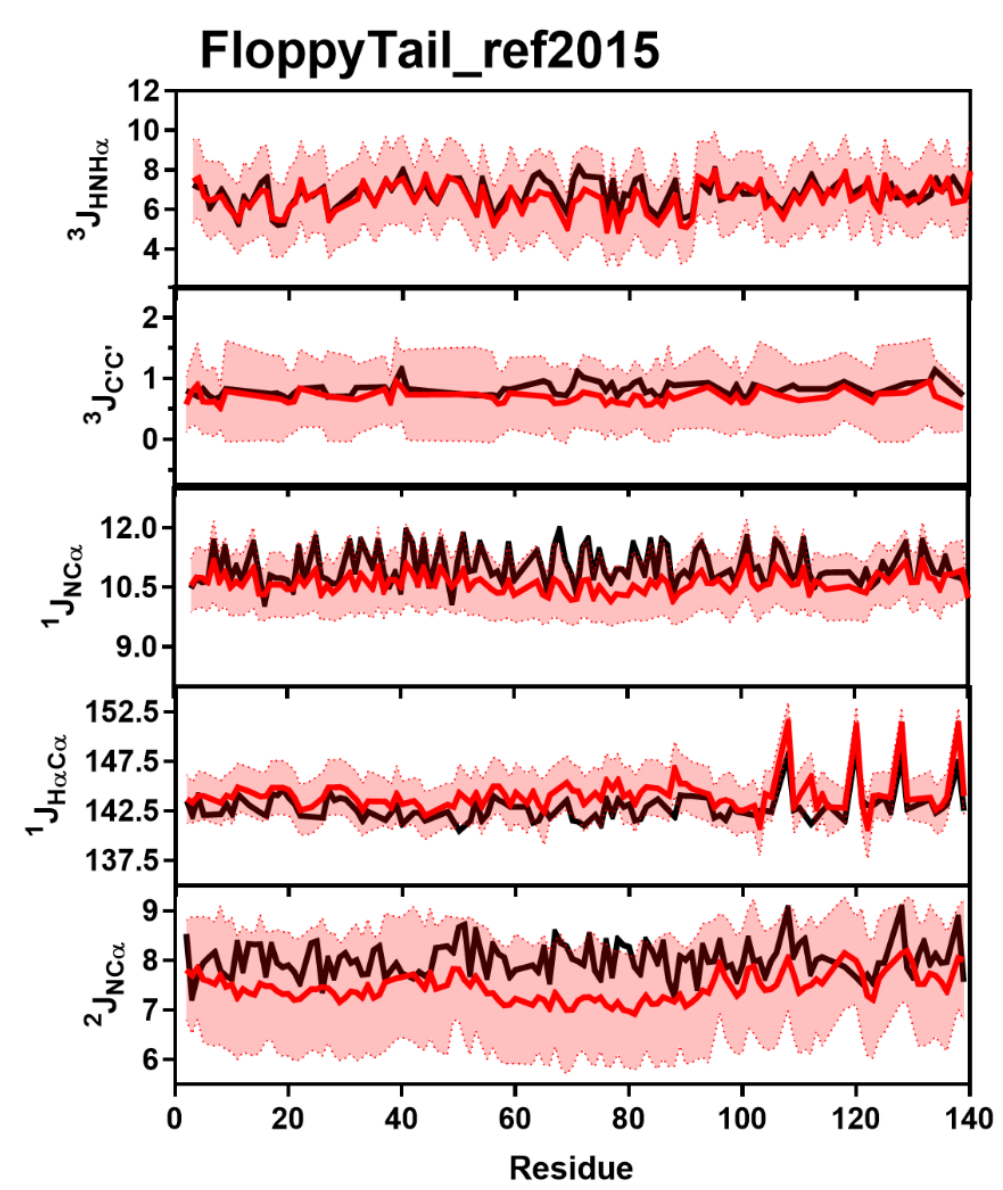

Figure S92: Comparison of Simulated and Experimental J-Coupling Data. Simulated J-Coupling values from the FloppyTail_ref2015 ensemble (red) overlayed on experimental data (black) of ${ }^{3} \mathrm{~J}_{\mathrm{HNH} \alpha}$ (Top), ${ }^{3} \mathrm{~J}_{\mathrm{CC}}$ (Upper Middle), ${ }^{1} \mathrm{~J}_{\mathrm{H} \alpha \mathrm{C} \alpha}$ (Middle), ${ }^{1} \mathrm{~J}_{\mathrm{NC} \alpha}$ (Lower Middle) and ${ }^{2} \mathrm{~J}_{\mathrm{NC} \alpha}$ (Bottom) from Mantsyzov et al. and Lee et al. ${ }^{13,18}$. 


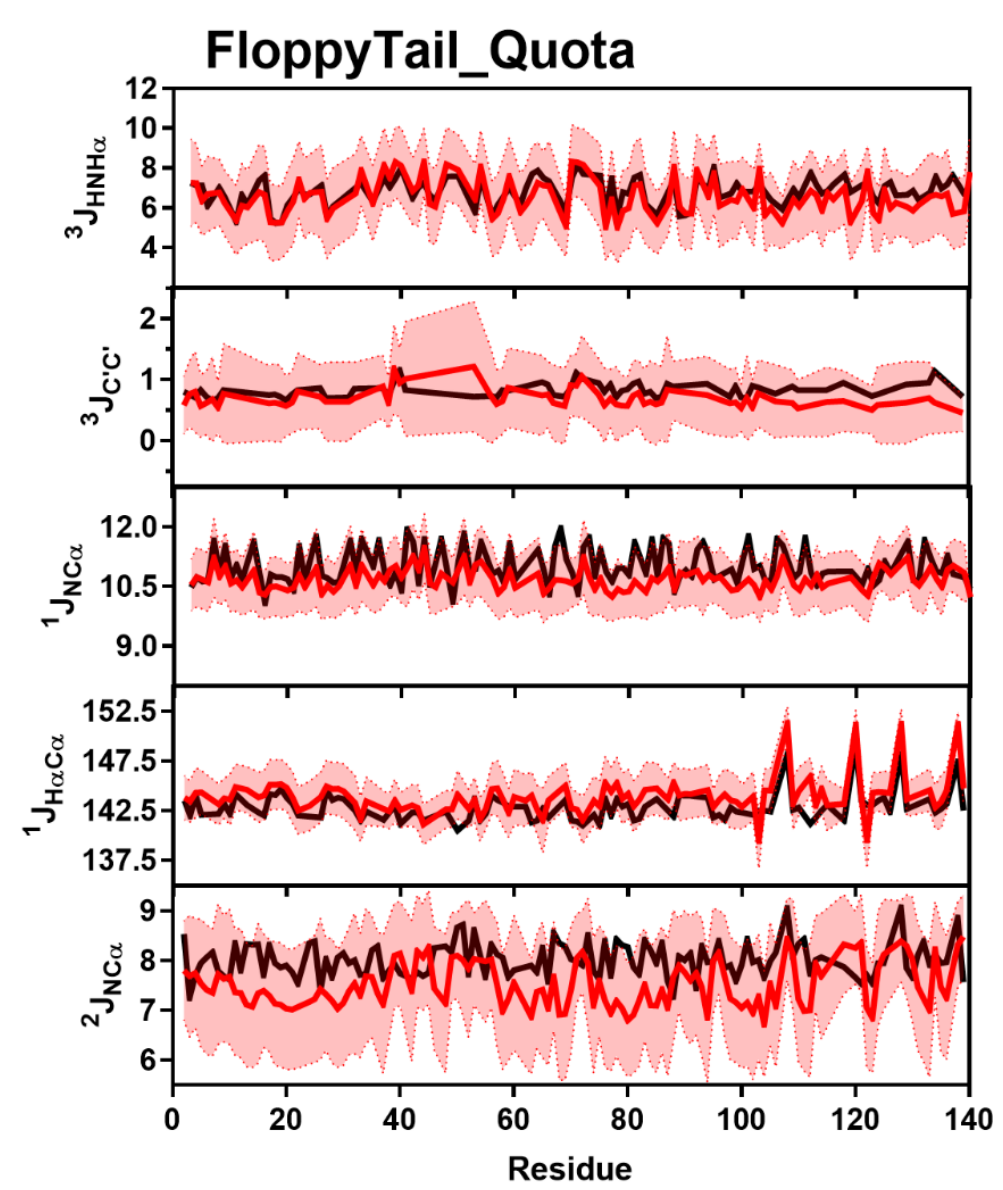

Figure S93: Comparison of Simulated and Experimental J-Coupling Data. Simulated J-Coupling values from the FloppyTail_Quota ensemble (red) overlayed on experimental data (black) of ${ }^{3} \mathrm{~J}_{\mathrm{HNH} \alpha}$ (Top), ${ }^{3} \mathrm{~J}_{\mathrm{CC}}$ (Upper Middle), ${ }^{1} \mathrm{~J}_{\mathrm{H} \alpha \mathrm{C} \alpha}$ (Middle), ${ }^{1} \mathrm{~J}_{\mathrm{NC} \alpha}$ (Lower Middle) and ${ }^{2} \mathrm{~J}_{\mathrm{NC} \alpha}$ (Bottom) from Mantsyzov et al. and Lee et al. ${ }^{13,18}$. 


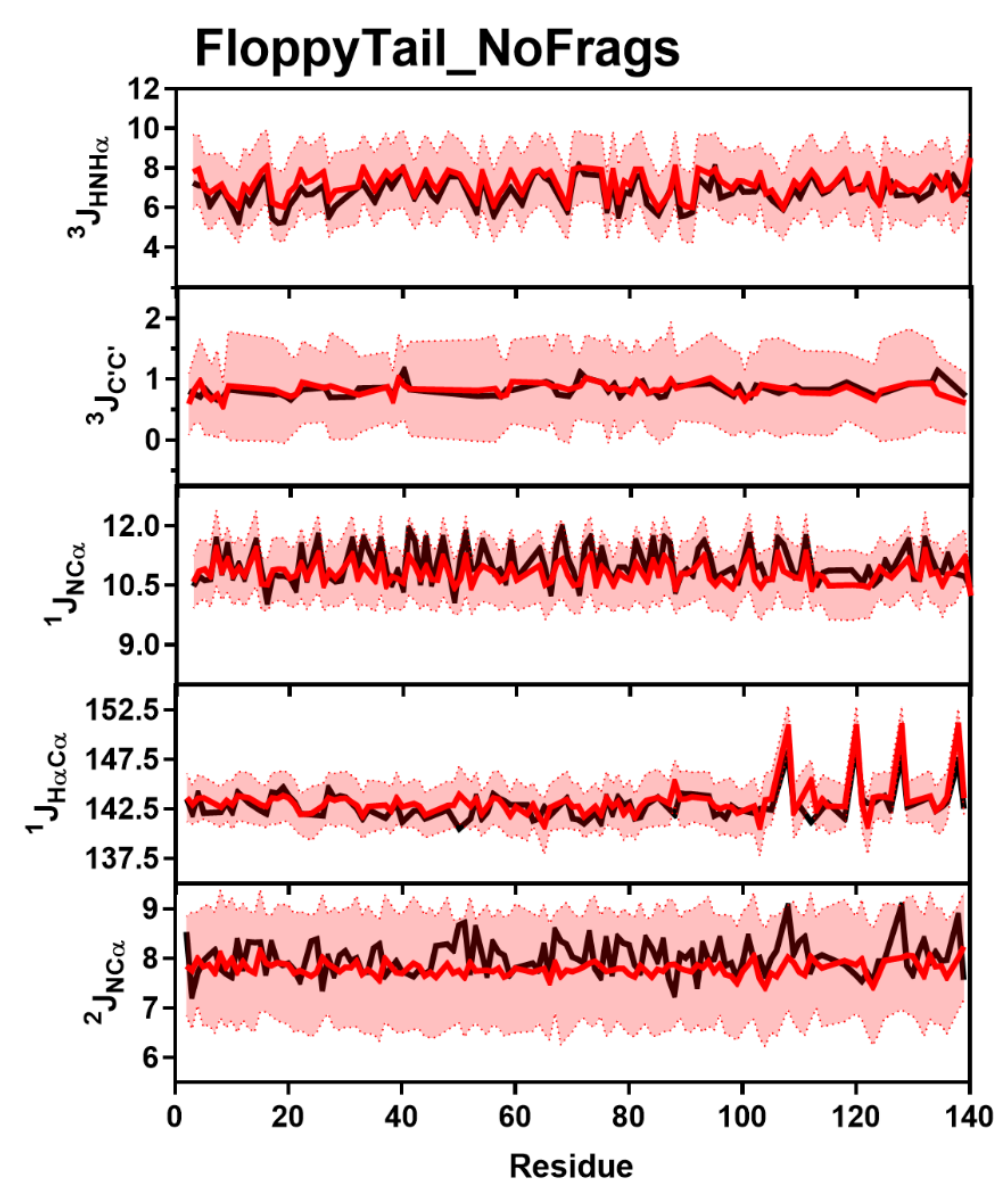

Figure S94: Comparison of Simulated and Experimental J-Coupling Data. Simulated J-Coupling values from the FloppyTail_NoFrags ensemble (red) overlayed on experimental data (black) of ${ }^{3} \mathrm{~J}_{\mathrm{HNH} \alpha}$ (Top), ${ }^{3} \mathrm{~J}_{\mathrm{CC}}$ (Upper Middle), ${ }^{1} \mathrm{~J}_{\mathrm{H} \alpha \mathrm{C} \alpha}$ (Middle), ${ }^{1} \mathrm{~J}_{\mathrm{NC} \alpha}$ (Lower Middle) and ${ }^{2} \mathrm{~J}_{\mathrm{NC} \alpha}$ (Bottom) from Mantsyzov et al. and Lee et al. ${ }^{13,18}$. 


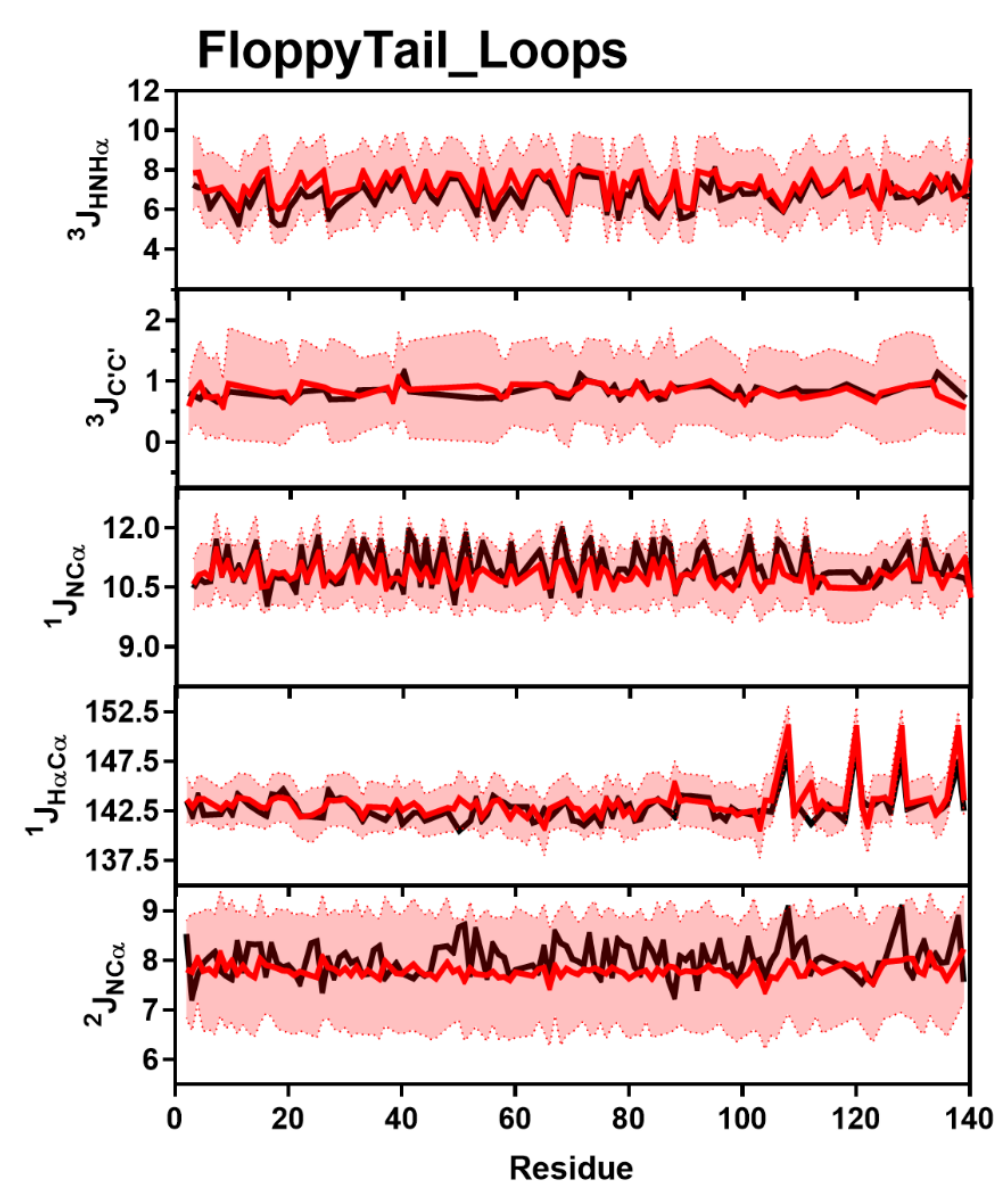

Figure S95: Comparison of Simulated and Experimental J-Coupling Data. Simulated J-Coupling values from the FloppyTail_Loops ensemble (red) overlayed on experimental data (black) of ${ }^{3} \mathrm{~J}_{\mathrm{HNH} \alpha}$ (Top), ${ }^{3} \mathrm{~J}_{\mathrm{CC}}$ (Upper Middle), ${ }^{1} \mathrm{~J}_{\mathrm{H} \alpha \mathrm{C} \alpha}$ (Middle), ${ }^{1} \mathrm{~J}_{\mathrm{NC} \alpha}$ (Lower Middle) and ${ }^{2} \mathrm{~J}_{\mathrm{NC} \alpha}$ (Bottom) from Mantsyzov et al. and Lee et al. ${ }^{13,18}$. 


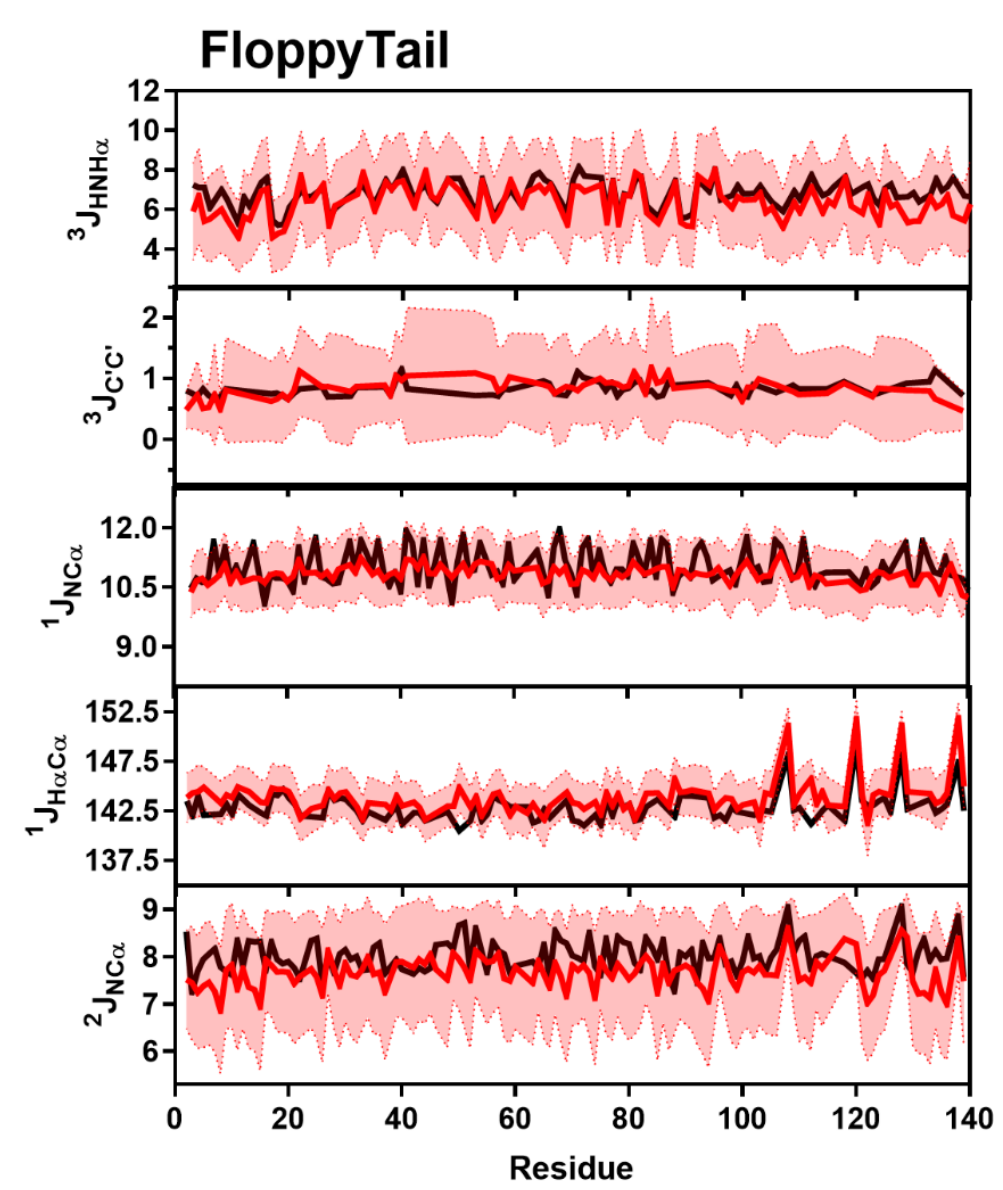

Figure S96: Comparison of Simulated and Experimental J-Coupling Data. Simulated J-Coupling values from the FloppyTail ensemble (red) overlayed on experimental data (black) of ${ }^{3} \mathrm{~J}_{\mathrm{HNH} \alpha}$ (Top), ${ }^{3} \mathrm{~J}_{\mathrm{CC}}$ (Upper Middle), ${ }^{1} \mathrm{~J}_{\mathrm{H} \alpha \mathrm{C} \alpha}$ (Middle), ${ }^{1} \mathrm{~J}_{\mathrm{NC} \alpha}$ (Lower Middle) and ${ }^{2} \mathrm{~J}_{\mathrm{NC} \alpha}$ (Bottom) from Mantsyzov et al. and Lee et al. ${ }^{13,18}$. 


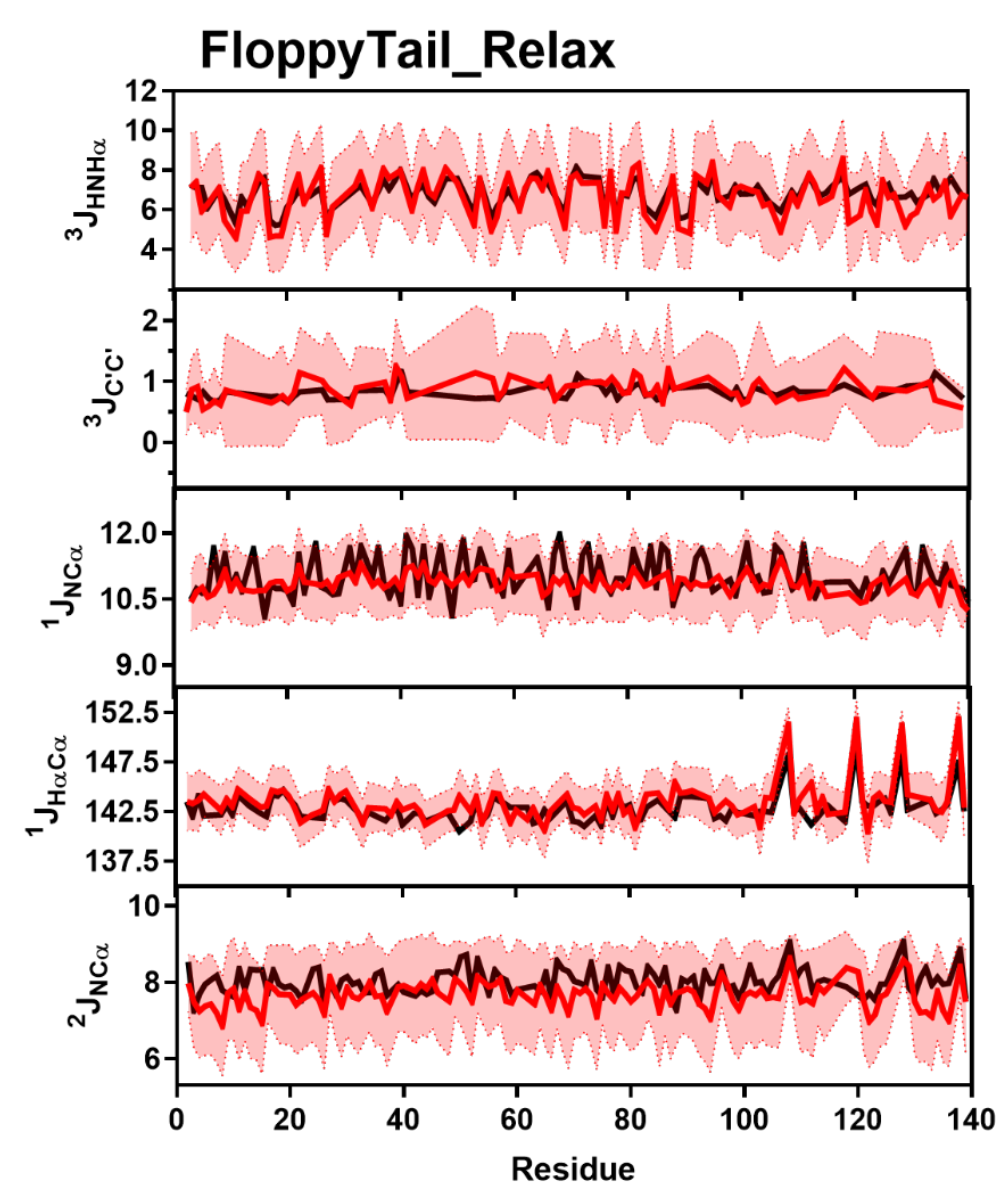

Figure S97: Comparison of Simulated and Experimental J-Coupling Data. Simulated J-Coupling values from the FloppyTail_Relax ensemble (red) overlayed on experimental data (black) of ${ }^{3} \mathrm{~J}_{\mathrm{HNH} \alpha}$ (Top), ${ }^{3} \mathrm{~J}_{\mathrm{CC}}$ (Upper Middle), ${ }^{1} \mathrm{~J}_{\mathrm{H} \alpha \mathrm{C} \alpha}$ (Middle), ${ }^{1} \mathrm{~J}_{\mathrm{NC} \alpha}$ (Lower Middle) and ${ }^{2} \mathrm{~J}_{\mathrm{NC} \alpha}$ (Bottom) from Mantsyzov et al. and Lee et al. ${ }^{13,18}$. 


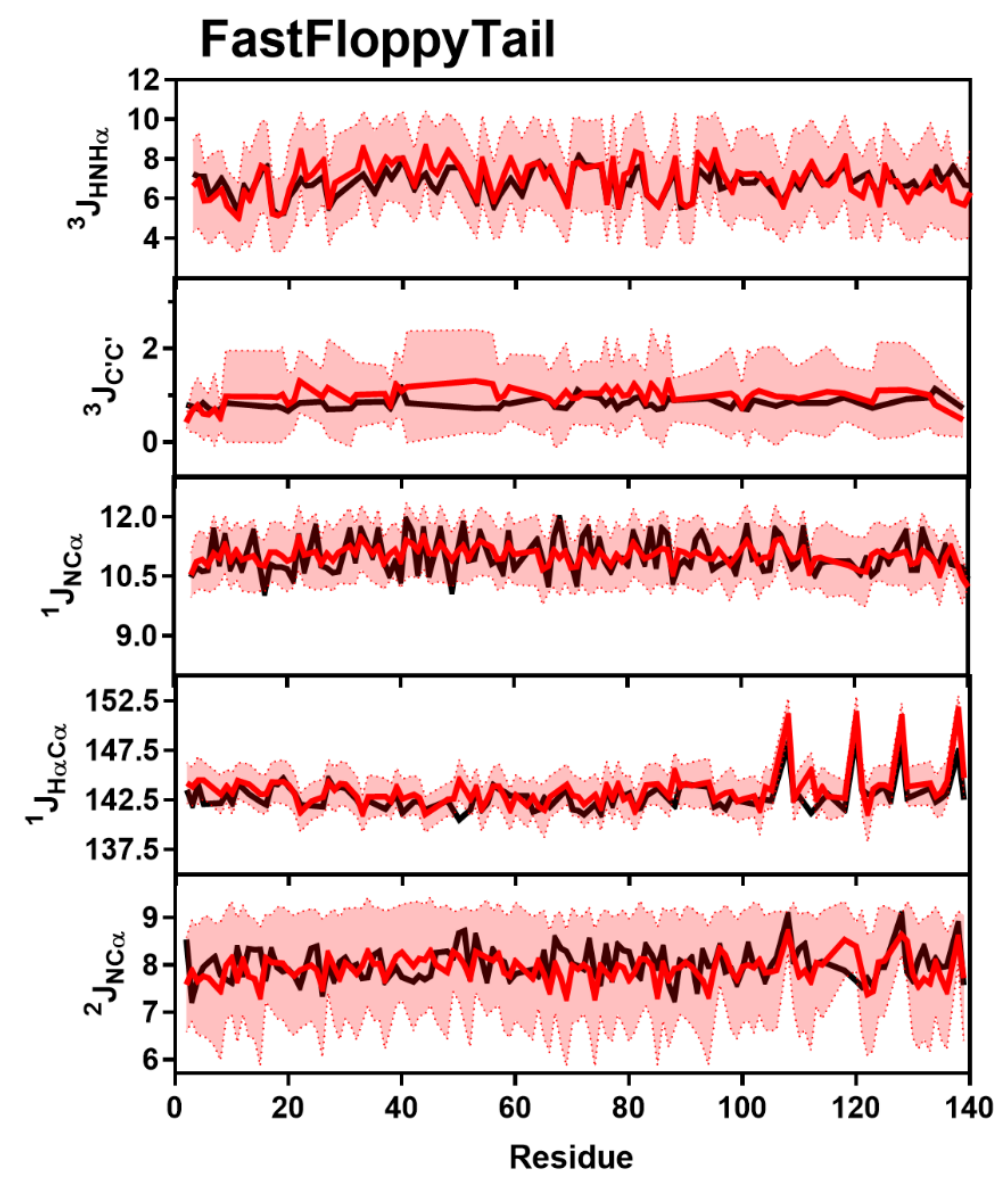

Figure S98: Comparison of Simulated and Experimental J-Coupling Data. Simulated J-Coupling values from the FastFloppyTail ensemble (red) overlayed on experimental data (black) of ${ }^{3} \mathrm{~J}_{\mathrm{HNH} \alpha}$ (Top), ${ }^{3} \mathrm{~J}_{\mathrm{CC}}$ (Upper Middle), ${ }^{1} \mathrm{~J}_{\mathrm{H} \alpha \mathrm{C} \alpha}$ (Middle), ${ }^{1} \mathrm{~J}_{\mathrm{NC} \alpha}$ (Lower Middle) and ${ }^{2} \mathrm{~J}_{\mathrm{NC} \alpha}$ (Bottom) from Mantsyzov et al. and Lee et al. ${ }^{13,18}$. 


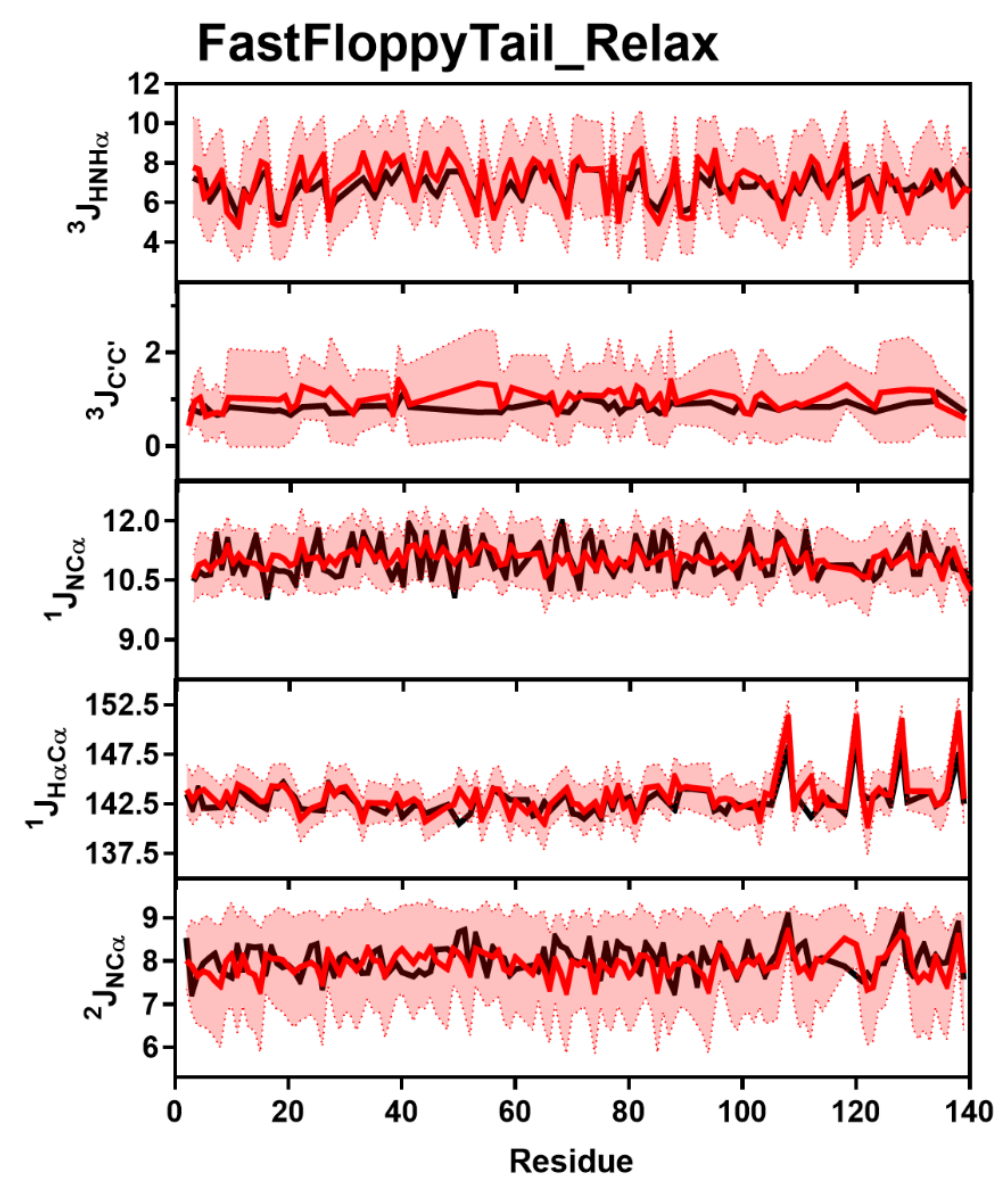

Figure S99: Comparison of Simulated and Experimental J-Coupling Data. Simulated J-Coupling values from the FastFloppyTail_Relax ensemble (red) overlayed on experimental data (black) of ${ }^{3} \mathrm{~J}_{\mathrm{HNH} \alpha}$ (Top), ${ }^{3} \mathrm{~J}_{\mathrm{CC}}$ (Upper Middle), ${ }^{1} \mathrm{~J}_{\mathrm{H} \alpha \mathrm{C} \alpha}$ (Middle), ${ }^{1} \mathrm{~J}_{\mathrm{NC} \alpha}$ (Lower Middle) and ${ }^{2} \mathrm{~J}_{\mathrm{NC} \alpha}$ (Bottom) from Mantsyzov et al. and Lee et al. ${ }^{13,18}$. 


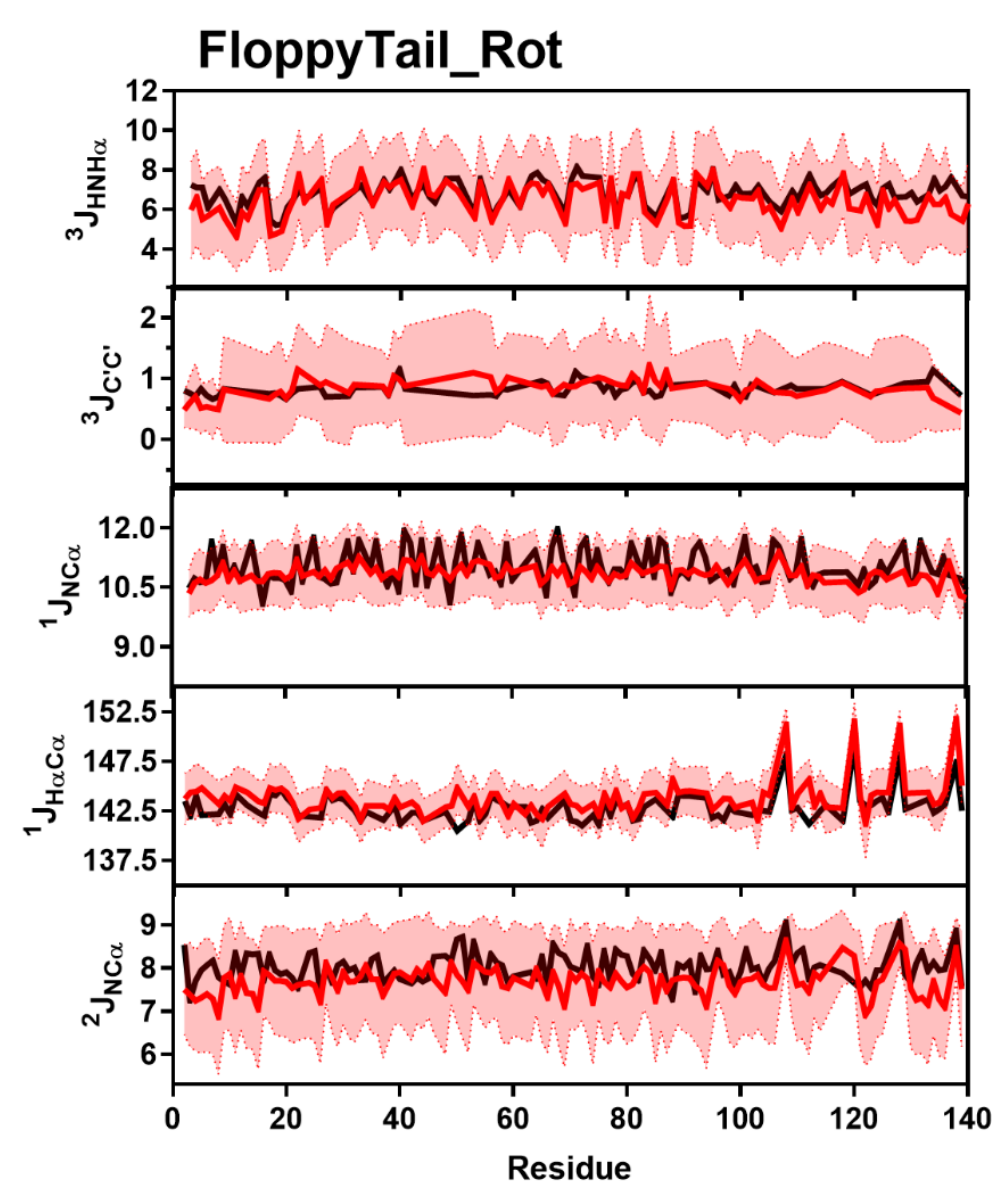

Figure S100: Comparison of Simulated and Experimental J-Coupling Data. Simulated J-Coupling values from the FloppyTail_Rot ensemble (red) overlayed on experimental data (black) of ${ }^{3} \mathrm{~J}_{\mathrm{HNH} \alpha}$ (Top), ${ }^{3} \mathrm{~J}_{\mathrm{CC}}$ (Upper Middle), ${ }^{1} \mathrm{~J}_{\mathrm{H} \alpha \mathrm{C} \alpha}$ (Middle), ${ }^{1} \mathrm{~J}_{\mathrm{NC} \alpha}$ (Lower Middle) and ${ }^{2} \mathrm{~J}_{\mathrm{NC} \alpha}$ (Bottom) from Mantsyzov et al. and Lee et al. ${ }^{13,18}$. 


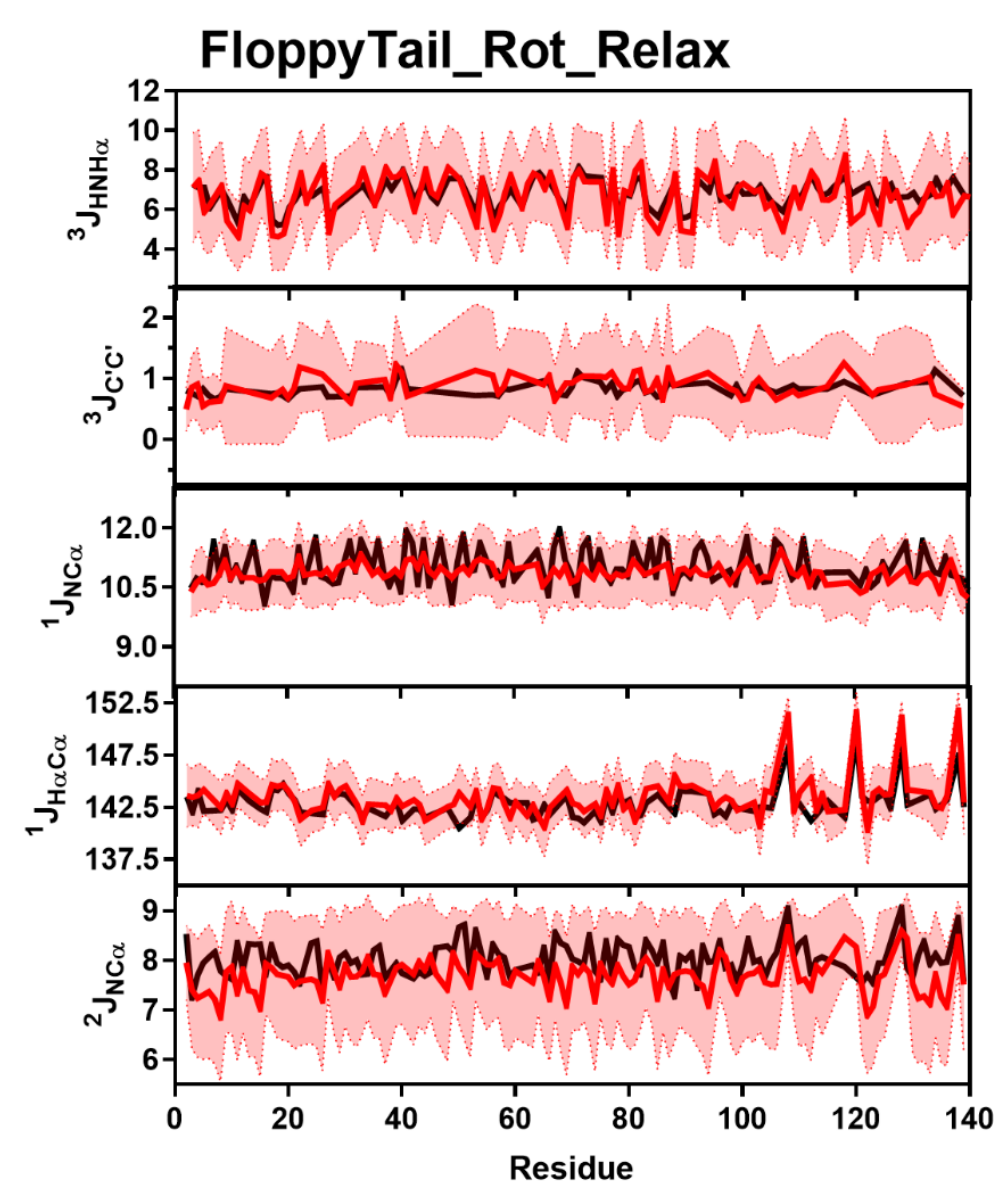

Figure S101: Comparison of Simulated and Experimental J-Coupling Data. Simulated J-Coupling values from the FloppyTail_Rot_Relax ensemble (red) overlayed on experimental data (black) of ${ }^{3} \mathrm{~J}_{\mathrm{HNH} \alpha}$ (Top), ${ }^{3} \mathrm{~J}_{\mathrm{CC}}$ (Upper Middle), ${ }^{1} \mathrm{~J}_{\mathrm{H} \alpha \mathrm{C} \alpha}$ (Middle), ${ }^{1} \mathrm{~J}_{\mathrm{NC} \alpha}$ (Lower Middle) and ${ }^{2} \mathrm{~J}_{\mathrm{NC} \alpha}$ (Bottom) from Mantsyzov et al. and Lee et al. ${ }^{13,18}$. 


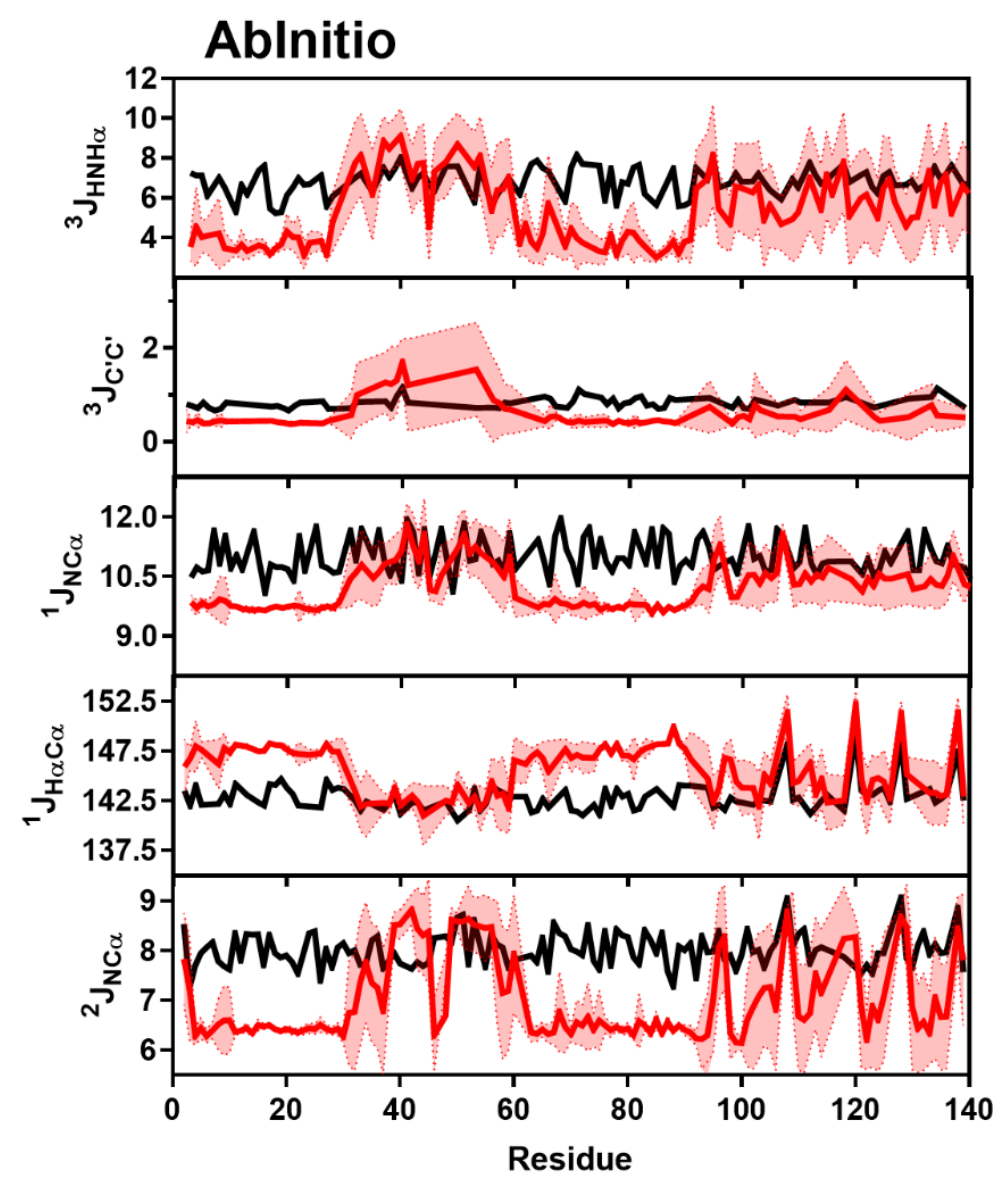

Figure S102: Comparison of Simulated and Experimental J-Coupling Data. Simulated J-Coupling values from the AbInitio ensemble (red) overlayed on experimental data (black) of ${ }^{3} \mathrm{~J}_{\mathrm{HNH} \alpha}$ (Top), ${ }^{3} \mathrm{~J}_{\mathrm{CC}}$ (Upper Middle), ${ }^{1} \mathrm{~J}_{\mathrm{H} \alpha \mathrm{C} \alpha}$ (Middle), ${ }^{1} \mathrm{~J}_{\mathrm{NC} \alpha}$ (Lower Middle) and ${ }^{2} \mathrm{~J}_{\mathrm{NC} \alpha}$ (Bottom) from Mantsyzov et al. and Lee et al. ${ }^{13,18}$. 


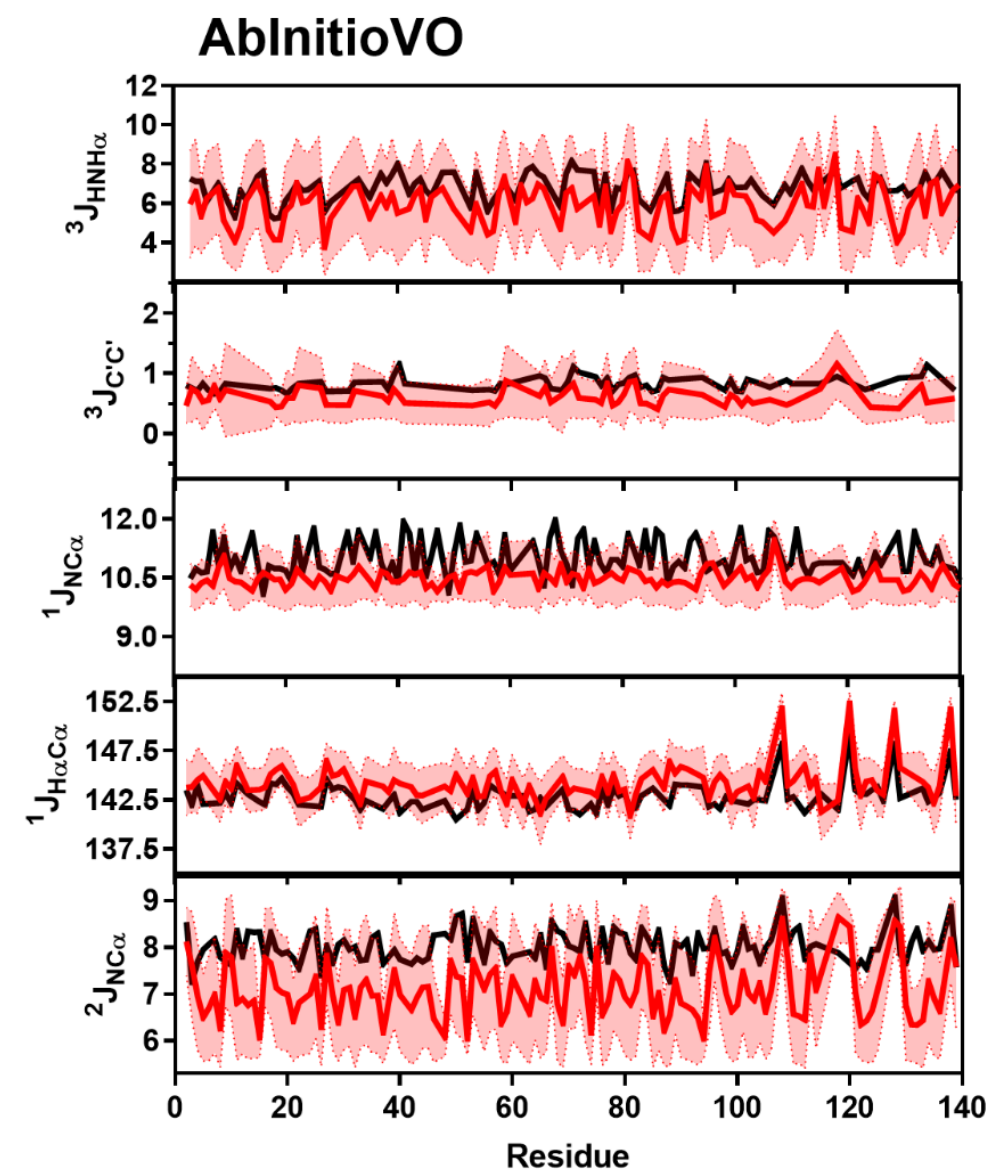

Figure S103: Comparison of Simulated and Experimental J-Coupling Data. Simulated J-Coupling values from the AbInitioVO ensemble (red) overlayed on experimental data (black) of ${ }^{3} \mathrm{~J}_{\mathrm{HNH} \alpha}$ (Top), ${ }^{3} \mathrm{~J}_{\mathrm{CC}}$ (Upper Middle), ${ }^{1} \mathrm{~J}_{\mathrm{H} \alpha \mathrm{C} \alpha}$ (Middle), ${ }^{1} \mathrm{~J}_{\mathrm{NC} \alpha}$ (Lower Middle) and ${ }^{2} \mathrm{~J}_{\mathrm{NC} \alpha}$ (Bottom) from Mantsyzov et al. and Lee et al. ${ }^{13,18}$. 
Construction of the New Rg Score Term: The previous "rg" centroid score term used by Rosetta is equal to a constant multiplied by the value of the radius of gyration of the protein at each step of the simulation. Therefore, even when a protein approaches its appropriate radius of gyration, this term continues to force compaction energetically. In our effort to abrogate this effect, which leads to the production of overly-compact conformations of disordered proteins, we have chosen to craft the potential in the form of a self-avoiding walk (SAW) probability distribution.

$$
\begin{gathered}
P(r)=A \frac{4 \pi}{\overline{R_{g}}}\left(\frac{r_{g}}{R_{g}}\right)^{2+g} \exp \left[-\alpha\left(\frac{r_{g}}{R_{g}}\right)^{\delta}\right] \\
g=(\gamma-1) / v \\
\delta=1 /(1-v) \\
\overline{R_{g}}=\sqrt{\frac{2 l_{p} b}{(2 v+1)(2 v+2)}} N^{v}
\end{gathered}
$$

Here, $r_{g}$ is the radius of gyration of the protein in a particular conformation, $\overline{R_{g}}$ is the average radius of gyration for a given protein, $v$ is the scaling exponent that, as exemplified in Eqn. S11, relates the number of residues in a protein $N$ to an average $R g$. The constants $b(0.38 \mathrm{~nm}), l_{p}(0.53$ $\mathrm{nm})$, and $\gamma(1.1615)$ have been previously estimated for proteins and the constants $A$ and $\alpha$ are determined for each set of $v$ and $\overline{R_{g}}$ through the conditions $\int_{0}^{\infty} P\left(r_{g}\right) d r_{g}=1$ and $\int_{0}^{\infty} P\left(r_{g}\right) r_{g}{ }^{2} d r={\overline{R_{g}}}^{2}{ }^{27-28}$. This provides for both a broad distribution of radii of gyration as well as an energetic barrier for overly compact states. The energy potential is defined as:

$$
E_{\overline{R_{g}}}\left(r_{g}\right)=1-^{P\left(r_{g}\right)} /_{\max }\left[P\left(r_{g}\right)\right]
$$

Through this form, the energy value scales from zero to one for any average radius of gyration allowing the weight of this score term within our overall score function to determine the depth of the potential.

To utilize this potential, a radius of gyration needs to be predicted from the primary sequence. Here, we utilize functional forms described in Hofmann et al. to predict the scaling exponent for a given protein based on the primary sequence composition and predict the radius of gyration using Eqn. S11 ${ }^{28}$. The scaling exponent can be predicted from either the net charge or hydrophobicity of the protein via the equations:

$$
\begin{gathered}
v(Q)=\frac{1}{3}+a\left[1+\frac{\exp \left(x_{0}-Q\right)}{z}\right]^{-1} \\
v(H)=\frac{1}{3}+a\left[1+\frac{\exp \left(x_{0}+c H-d\right)}{z}\right]^{-1}
\end{gathered}
$$


The parameters $a=0.394, z=0.009, x_{0}=0.114, c=1.72$ and $d=0.9$, were determined by fitting observed radii of gyration for a set of proteins in guanidinium hydrochloride to the above equations 28.

To select between the scaling factors computed by the net charge and hydrophobicity when computing an expected radius of gyration, the polyampholyte theory was applied to determine the effect of the net charge on the excluded volume of the system by computing the excess volume:

$$
v^{*}=\frac{4 \pi l_{B}(f-g)^{2}}{\kappa^{2}}-\frac{\pi l_{B}^{2}(f+g)^{2}}{\kappa}
$$

Here, $f$ and $g$ are the fraction of positively (Arg/Lys) and negatively (Glu/Asp) charged residues, respectively, $\kappa$ is the Debye length defined by $\kappa^{-1}=0.304 / \sqrt{I}$, where $I$ is the ionic strength and $l_{B}$ is the Bjerrum length defined by:

$$
l_{B}=e^{2} /\left(4 \pi \varepsilon_{0} \varepsilon_{r} k_{B} T\right)
$$

In the above equation, $e$ is the elementary charge $\left(1.602 \times 10^{-19}\right), \varepsilon_{0}$ is the dielectric constant ( 8.854 $\left.\times 10^{-12}\right), \varepsilon_{r}$ is the permittivity of water (78.7) and $k_{B}$ is the Boltzmann constant. In the general case for the calculation of $v^{*}$, the ionic strength and the temperature are set to $0.15 \mathrm{M}$ and $298 \mathrm{~K}$ respectively. Since values of $v^{*}$ greater than zero correspond to net electrostatic repulsion, when $v^{*}$ is greater than zero, the scaling term computed using the net charge is applied when predicting the radius of gyration. Conversely, when $v^{*}$ is less than zero, there is a net electrostatic attraction, forcing the scaling based on the hydrophobicity to dominate. In cases where both the fractions of positively and negatively charged residues are zero, the scaling is determined using the net hydrophobicity. Lastly, in the cases where either the fraction of positively or negatively charged residues equals zero, the scaling due to the net charge is selected.

Upon further inspection of the above method proposed by Hofmann et al., we noted that when testing this function for lysozyme (PDB: 2LZM) the predicted scaling value $(v=0.519)$ is significantly higher than the expected value for a folded protein ${ }^{28}$. Since the fitting was performed on denatured proteins, the work by Hofmann et al. shows that when determining the radius of gyration for a suite of proteins from the Protein Data Bank, the minimum observed scaling factor is not equal to 0.33 , which is the value for well-folded proteins, but instead is $\sim 0.4{ }^{28}$. Therefore, we utilized the per residue disordered probability of the protein to determine if a given segment of the protein of interest was folded or unfolded. The RaptorX server was used to obtain per residue disordered probabilities, where residues with a disordered probability $>0.5$ were determined to be disordered ${ }^{6}$. Segments were defined as any stretch of residues greater than 10 in which all residues were of the same classification, ordered or disordered. Segments deemed ordered were assigned a scaling value of 0.33 while the scaling value for disordered regions was computed using the above equations.

Implementation of the New Rg Score Term in PyRosetta: The updated "rg" term was implemented within PyRosetta by producing a new score term using the WholeStructureEnergy object. The annotated script (available upon email request to JJF or EJP) demonstrates how this is 
accomplished in detail, and the method is explained in brief below. After producing a pose from the input sequence, the net disordered probability was computed from the per residue disordered probability from RaptorX ${ }^{6}$. For net disordered probabilities below 0.5 , a scaling constant value of 0.33 was assigned. For all others, scaling values were computed from the net charge and hydrophobicitiy and a scaling constant value was selected using the effect of the net charge on the excluded volume as detailed above. After determining the value of the scaling constant, an expected average radius of gyration and radii of gyration probability distribution described by a self-avoiding random walk were computed. The values for $A$ and $\alpha$ were obtained using a discrete sum of distances from zero to $5 \times R_{g} \AA$ at a spacing of $0.01 \AA$. The probability distribution was converted in a potential energy function as described above and stored as the interpolated function after conversion using the scipy interp1d functionality ${ }^{29}$. Each time the pose was scored, the radius of gyration was computed using the neighbor atom coordinates and the value of the new radius of gyration score term value was assigned at each residue as the energy determined from the interpolated potential divided by the total number of residues. The weight of the score term within the score1-3 score functions was set at 80.0, which was determined to be optimal via the process detailed below.

Determination of the Optimal Weight for the New Rg Score Term: To determine the optimal weight of the new $R_{g}$ score term, $\alpha \mathrm{S}$ was simulated using a range of weighting values. We identified the optimal weighting value as that which maximized the impact of the score term on the average radius of gyration while minimizing the restriction on the conformational diversity. 


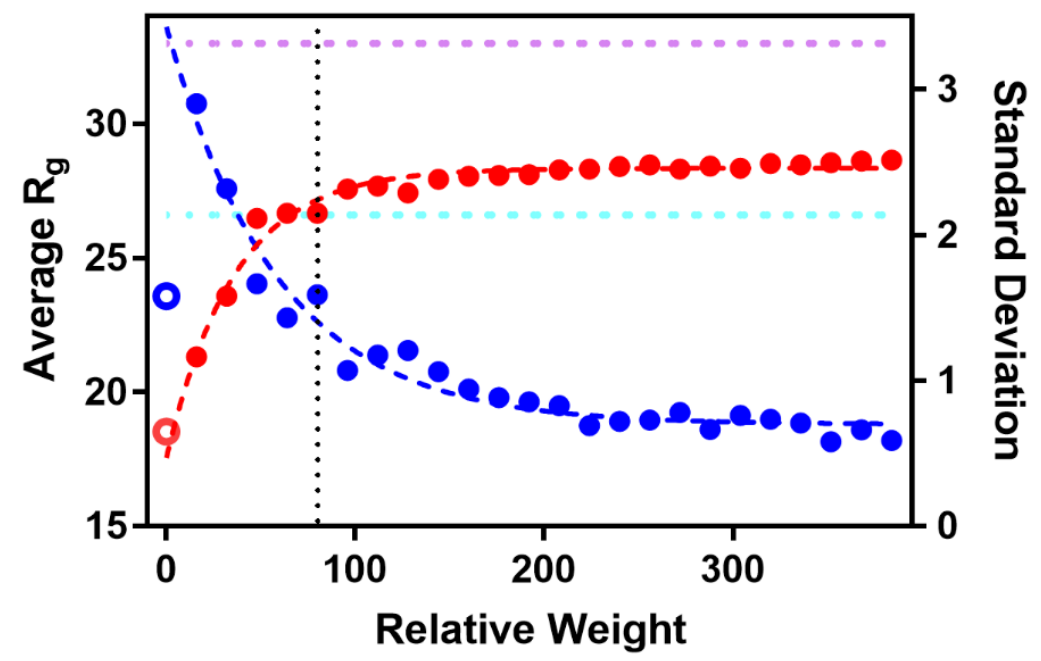

Figure S104: Determination of optimal new $R_{g}$ score term weight. Plot shows the average (filled blue circles) and standard deviation (filled red circles) radius of gyration resultant from simulations with various relative weights of the new $R_{g}$ score term compared to the simulation lacking the traditional rg term (open red and blue circles) and experimental data from SAXS (pink dashed line) and NMR (cyan dashed line). The vertical black dashed line illustrates the chosen optimal score term weight, as this pushes the average $R g$ closest to the experimental value without overconstraining the sampling. Therefore, the weights of all terms in the new score 3 term is equivalent to the traditional score 3 term, with the canonical rg term set to weight a zero and the weight of this new term set to 84 .

\section{Comparison of AbInitio and AbInitioVO Simulations to Experimental Data}

\section{Simulated Proteins and Abbreviations}

The 25-protein test set to assessing the accuracy of AbInitioVO compared to AbInito consisted of anti-HIV protein AOP-RANTES (1b3a), $\alpha$-spectrin SH3 domain D46G mutant (1bk2), Rubredoxin from Pyrococcus Furiosus (1bq9), Drosophila engrailed homeodomain (1 enh), Protein L B1 domain from Peptosteptococcus Magnus (1hz6), B2 immunoglobulin-binding domain of Streptococcal Protein G (1pgx), amino-terminal domain of phage 434 repressor (1r69), Human FYN SH3 domain (1shf), ubiquitin (1ubi), CRO repressor protein from bacteriophage lambda (5cro), Human translation initiation factor EIF1A (1d7q), Human co-chaperone P23 (1ejf), Cterminal domain of 50S ribosomal protein L11 (1fox), histone H1 (1ghc), HMGB protein NHP6A (1lwm), subunit F6 from the peripheral stalk region of ATP synthase (1vzs), Rap1 BRCT domain from Saccharomyces Cerevisiae (2142), yeast Tah1 (2lsu), $\alpha$-synuclein ( $\alpha \mathrm{S}$ or asyn), Drosophila adaptor protein Drk N-terminal SH3 domain (dsh3), C-terminal domain of nucleoprotein of measles virus (ntal), PaaA2 antitoxin from human pathogen E. coli $\mathrm{O} 157$ (paaa), cyclin-dependent kinase inhibitor Sic1 (sic1), and microtubule-associated protein tau K18 isoform (tauk). 


\section{Summary of Simulated Proteins}

Table S5: Comparison of AbInitio and AbInitioVO Outputs to PDB Structures

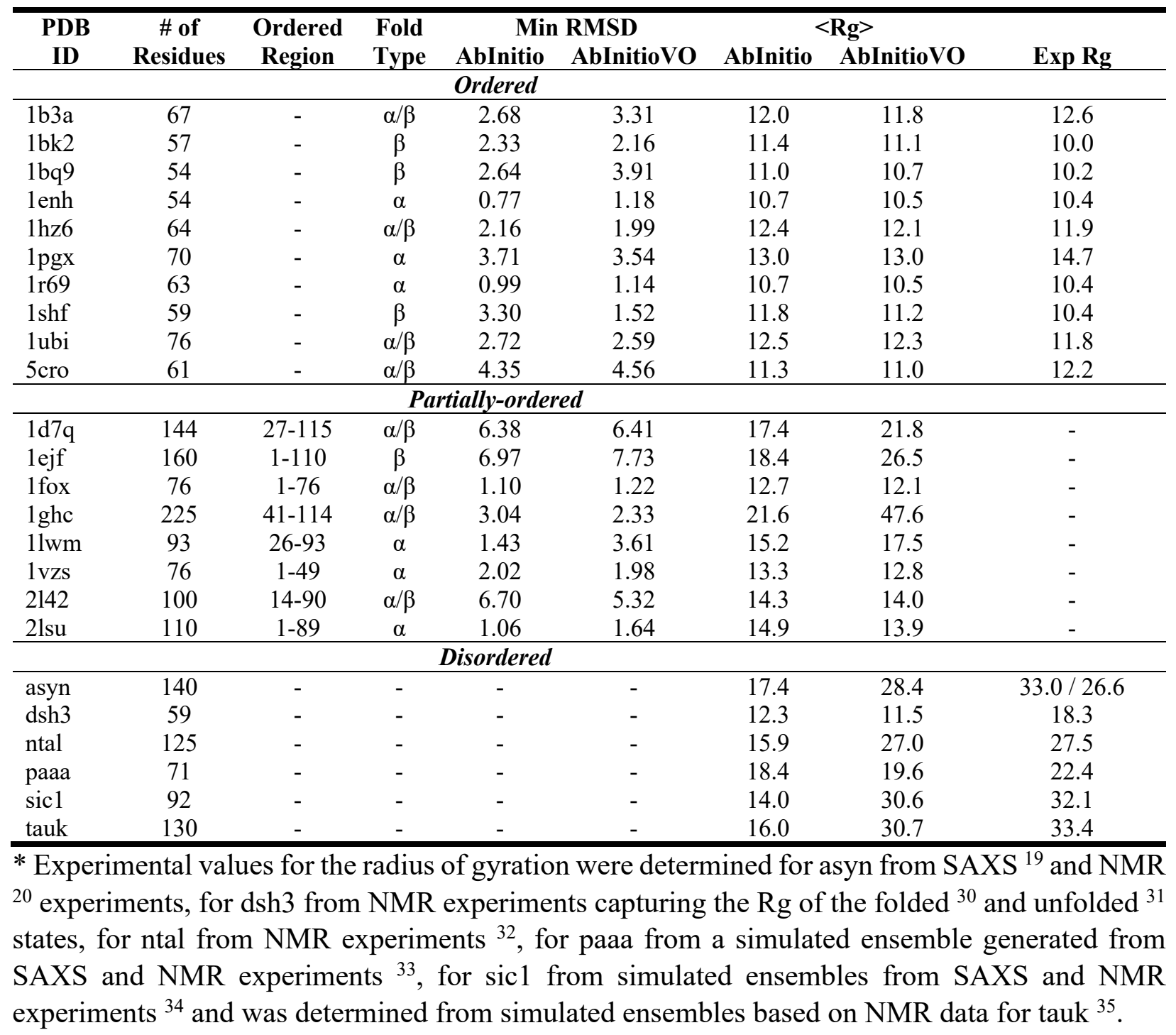




\section{Comparison of Radii of Gyration:}

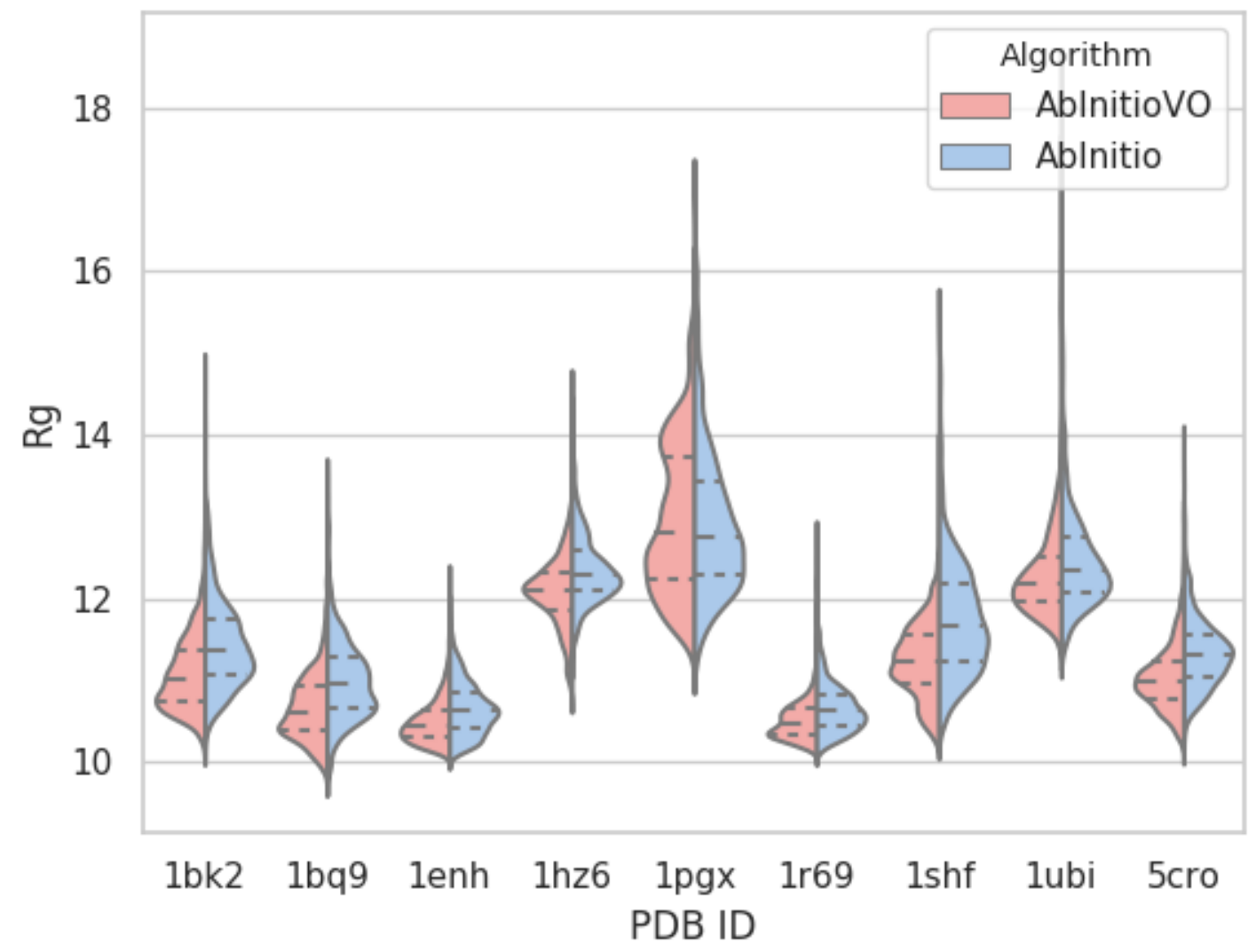

Figure S105: Radii of Gyration of Ordered Proteins. Violin plots show histogram for each simulation protein, as indicated by the x-axis, for all output from the AbInitioVO (red, left) and AbInitio (blue, right) simulations. Dashed lines on each violin represent the upper and lower quartile and the mean Rg. 


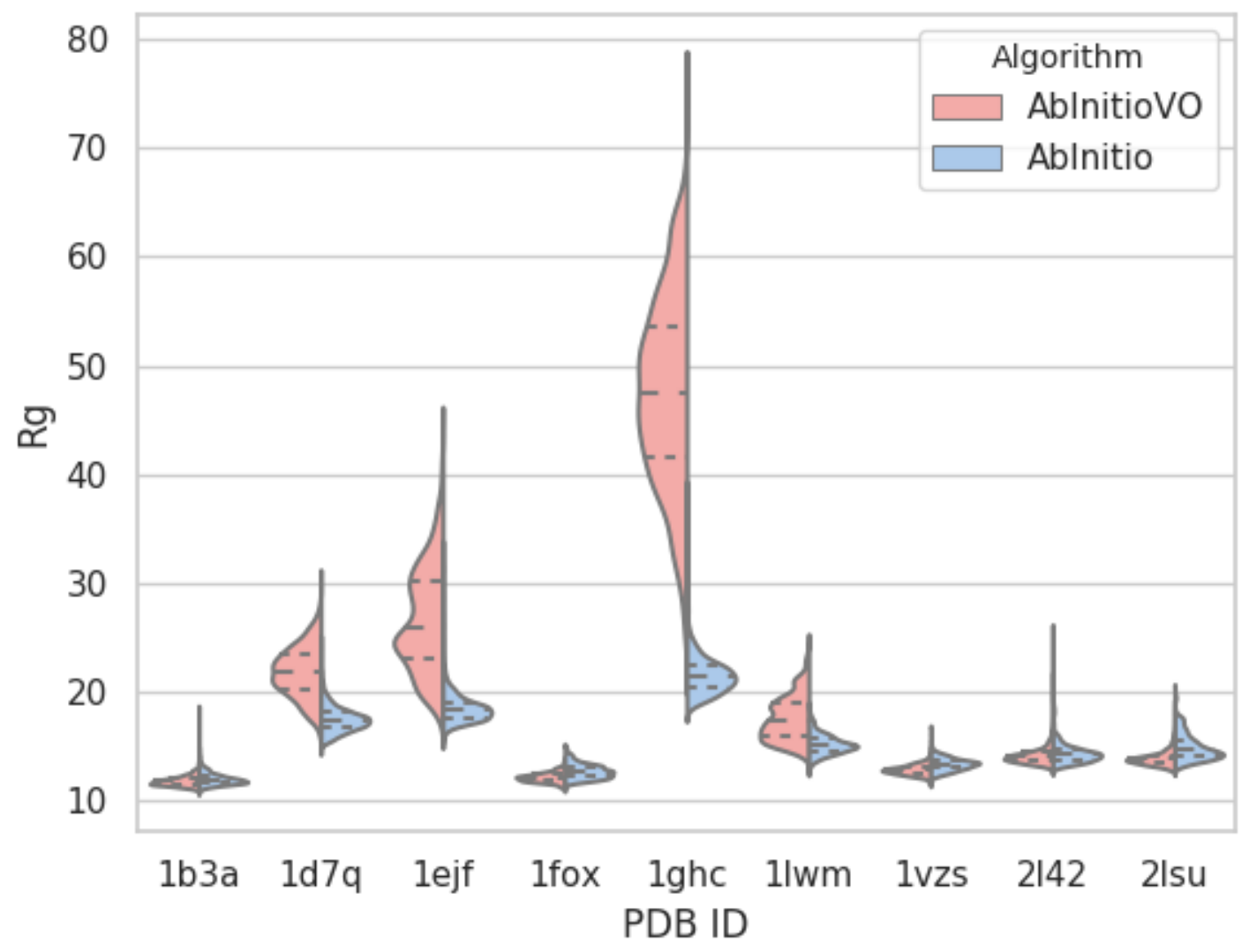

Figure S106: Radii of Gyration of Partially-Ordered Proteins. Violin plots show histogram for each simulation protein, as indicated by the x-axis, for all output from the AbInitioVO (red, left) and AbInitio (blue, right) simulations. Dashed lines on each violin represent the upper and lower quartile and the mean Rg. 


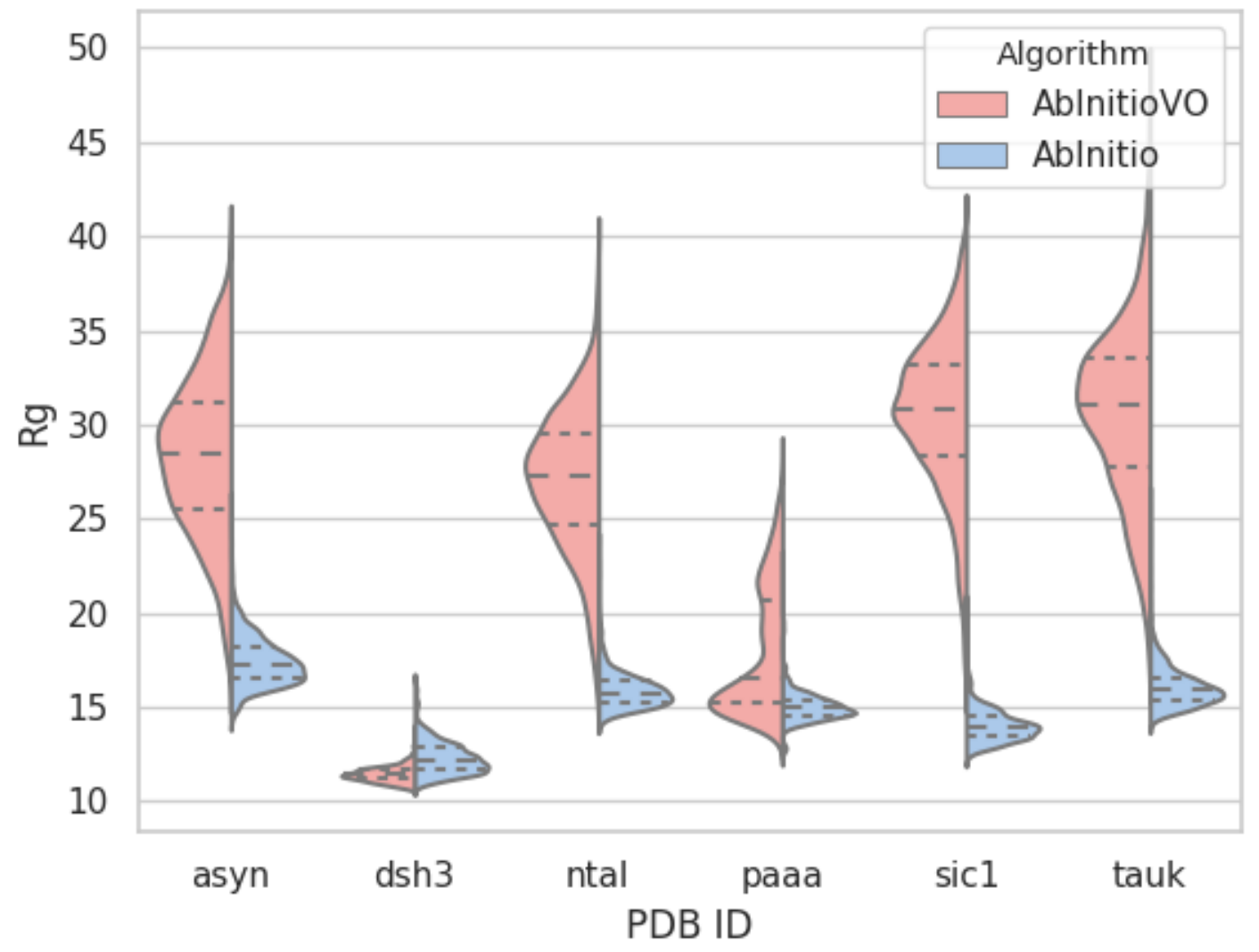

Figure S107: Radii of Gyration of Disordered Proteins. Violin plots show histogram for each simulation protein, as indicated by the x-axis, for all output from the AbInitioVO (red, left) and AbInitio (blue, right) simulations. Dashed lines on each violin represent the upper and lower quartile and the mean Rg. 


\section{Comparison of Folding Funnels}
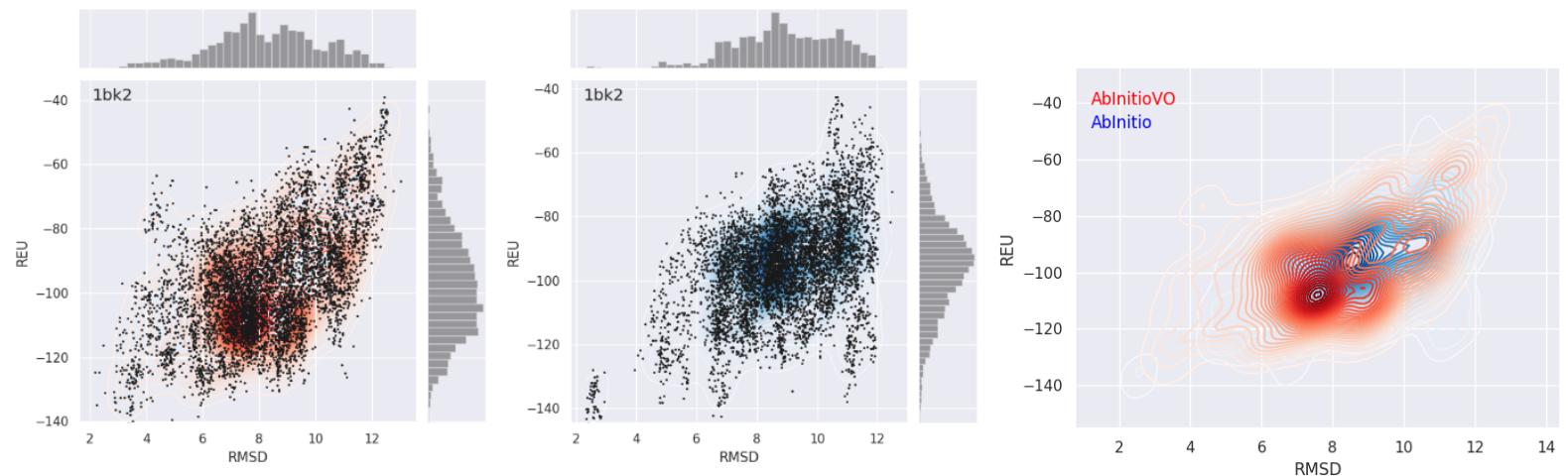

Figure S108: Folding Funnel Comparison for 1bka. Folding funnel from AbInitioVO (left) and AbInitio (middle) simulations plotting C $\alpha$ RMSD versus Rosetta Energy Units (REU) from each structure compared to the PDB structure. For each folding funnel, histograms of the computed RMSD (top) and REU (right) are shown. KDE plot (right) showing overlay of AbInitiovo (red) and AbInitio (blue) folding funnels.
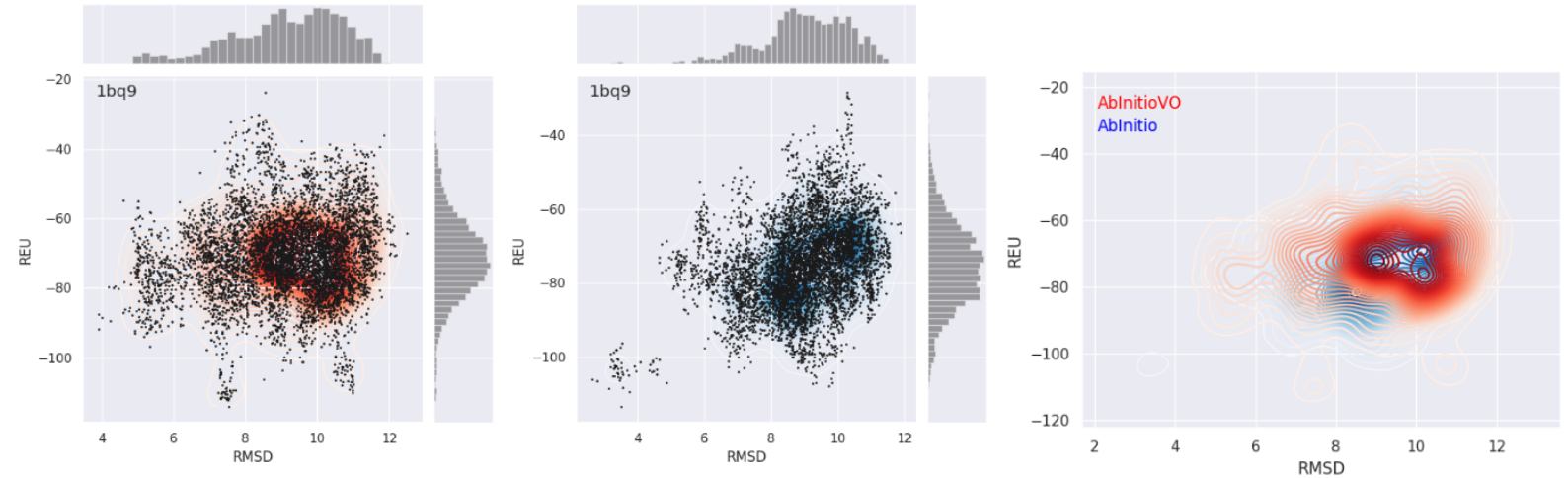

Figure S109: Folding Funnel Comparison for 1bq9. Folding funnel from AbInitioVO (left) and AbInitio (middle) simulations plotting C $\alpha$ RMSD versus Rosetta Energy Units (REU) from each structure compared to the PDB structure. For each folding funnel, histograms of the computed RMSD (top) and REU (right) are shown. KDE plot (right) showing overlay of AbInitiovo (red) and AbInitio (blue) folding funnels. 

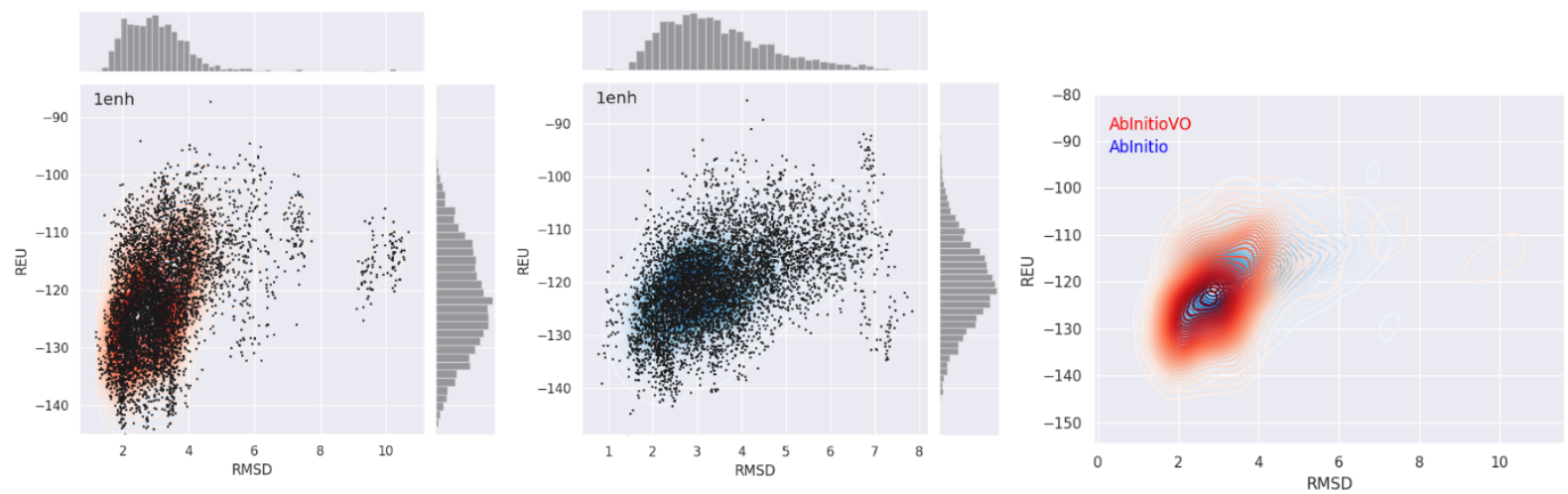

Figure S110: Folding Funnel Comparison for 1enh. Folding funnel from AbInitioVO (left) and AbInitio (middle) simulations plotting C $\alpha$ RMSD versus Rosetta Energy Units (REU) from each structure compared to the PDB structure. For each folding funnel, histograms of the computed RMSD (top) and REU (right) are shown. KDE plot (right) showing overlay of AbInitiovo (red) and AbInitio (blue) folding funnels.
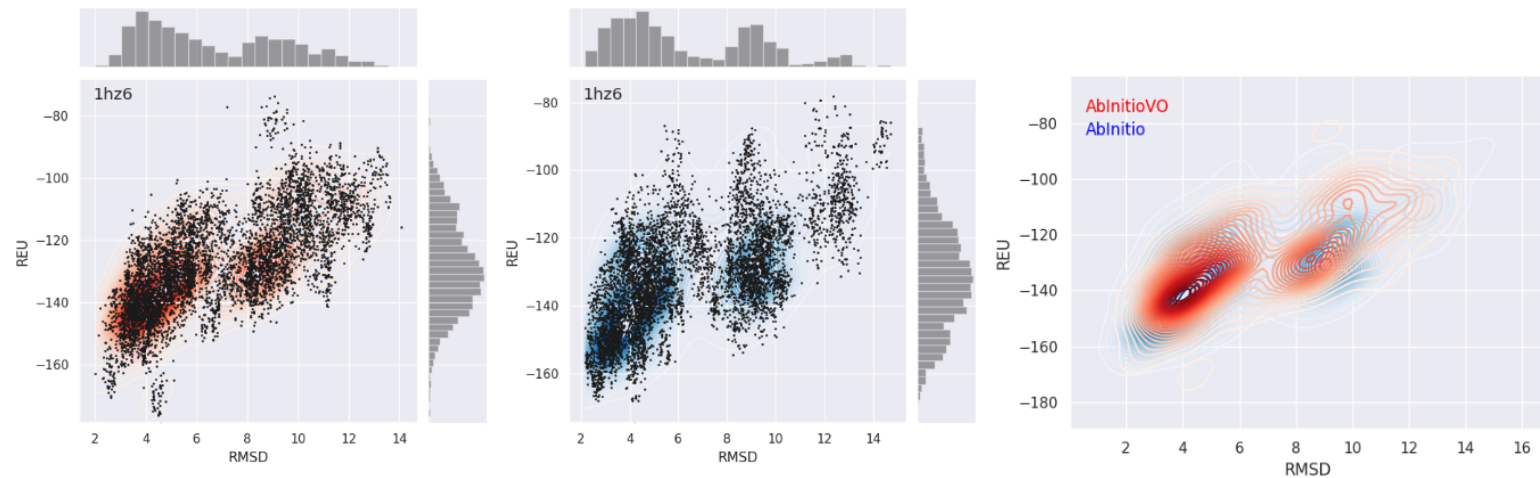

Figure S111: Folding Funnel Comparison for 1hz6. Folding funnel from AbInitioVO (left) and AbInitio (middle) simulations plotting C $\alpha$ RMSD versus Rosetta Energy Units (REU) from each structure compared to the PDB structure. For each folding funnel, histograms of the computed RMSD (top) and REU (right) are shown. KDE plot (right) showing overlay of AbInitiovo (red) and AbInitio (blue) folding funnels. 

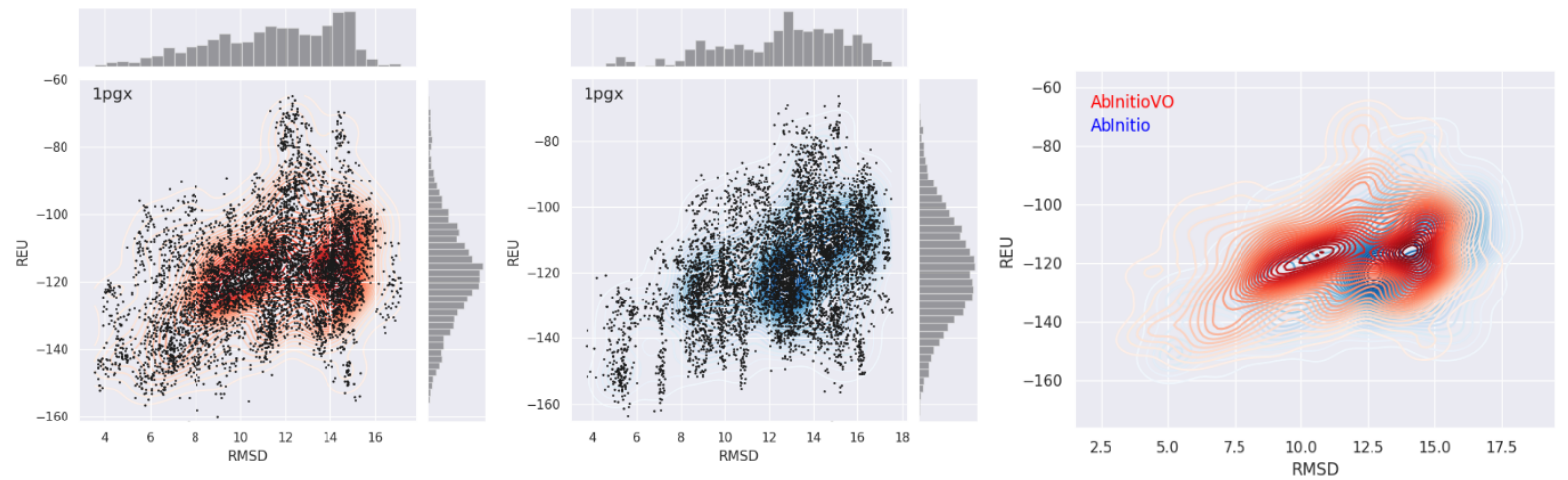

Figure S112: Folding Funnel Comparison for 1pgx. Folding funnel from AbInitioVO (left) and AbInitio (middle) simulations plotting C $\alpha$ RMSD versus Rosetta Energy Units (REU) from each structure compared to the PDB structure. For each folding funnel, histograms of the computed RMSD (top) and REU (right) are shown. KDE plot (right) showing overlay of AbInitiovo (red) and AbInitio (blue) folding funnels.
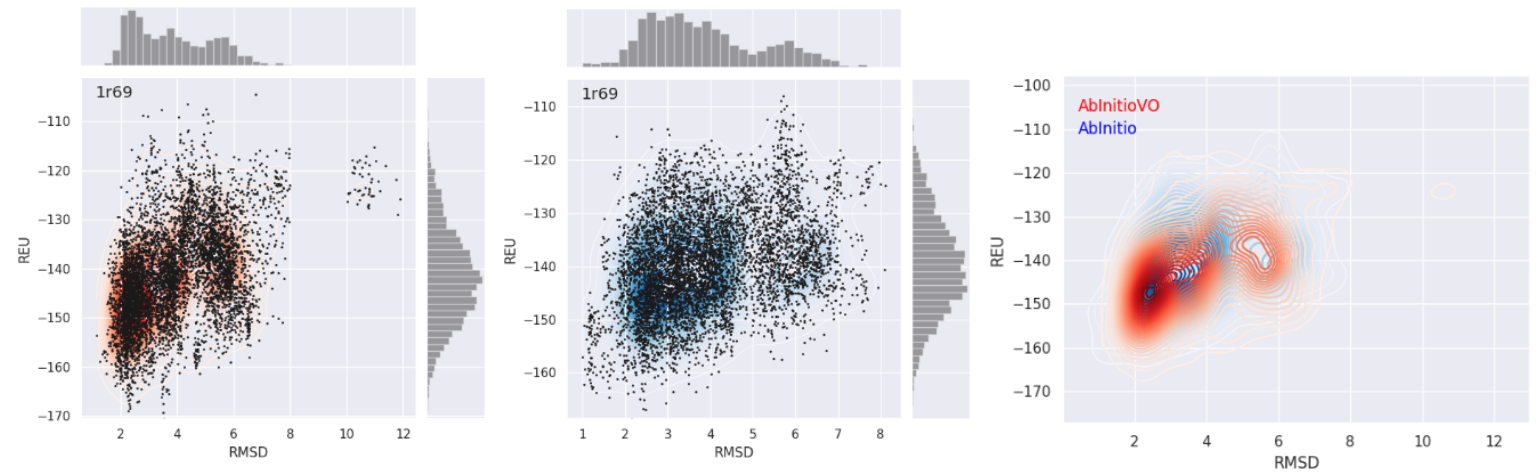

Figure S113: Folding Funnel Comparison for 1r69. Folding funnel from AbInitioVO (left) and AbInitio (middle) simulations plotting C $\alpha$ RMSD versus Rosetta Energy Units (REU) from each structure compared to the PDB structure. For each folding funnel, histograms of the computed RMSD (top) and REU (right) are shown. KDE plot (right) showing overlay of AbInitiovo (red) and AbInitio (blue) folding funnels. 

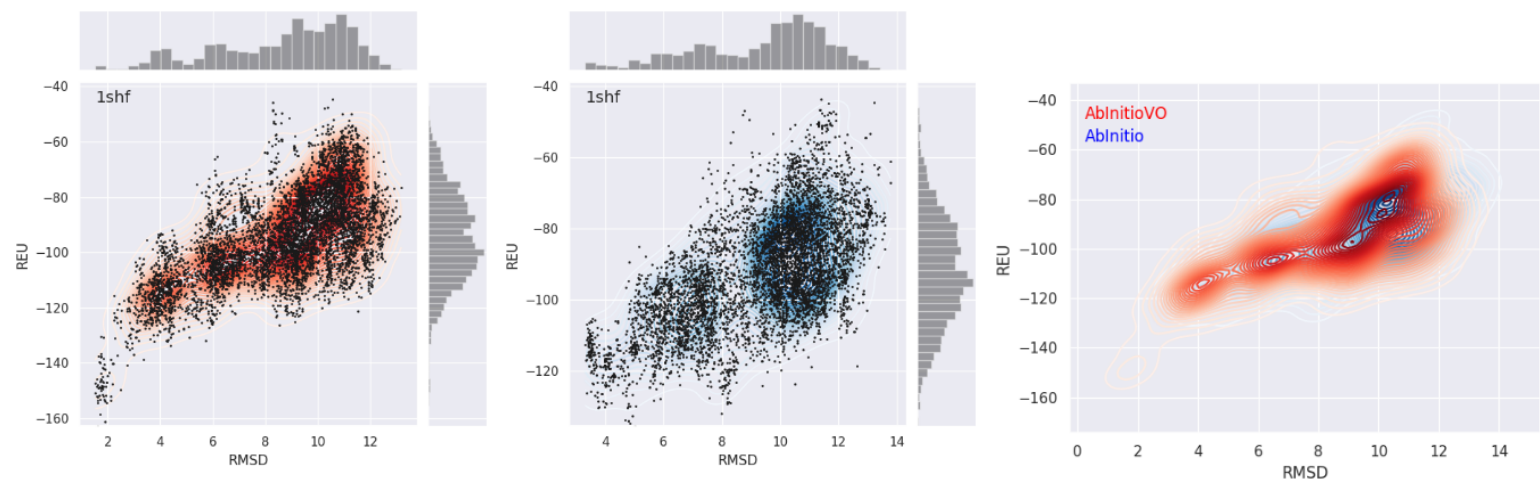

Figure S114: Folding Funnel Comparison for 1shf. Folding funnel from AbInitioVO (left) and AbInitio (middle) simulations plotting C $\alpha$ RMSD versus Rosetta Energy Units (REU) from each structure compared to the PDB structure. For each folding funnel, histograms of the computed RMSD (top) and REU (right) are shown. KDE plot (right) showing overlay of AbInitiovo (red) and AbInitio (blue) folding funnels.
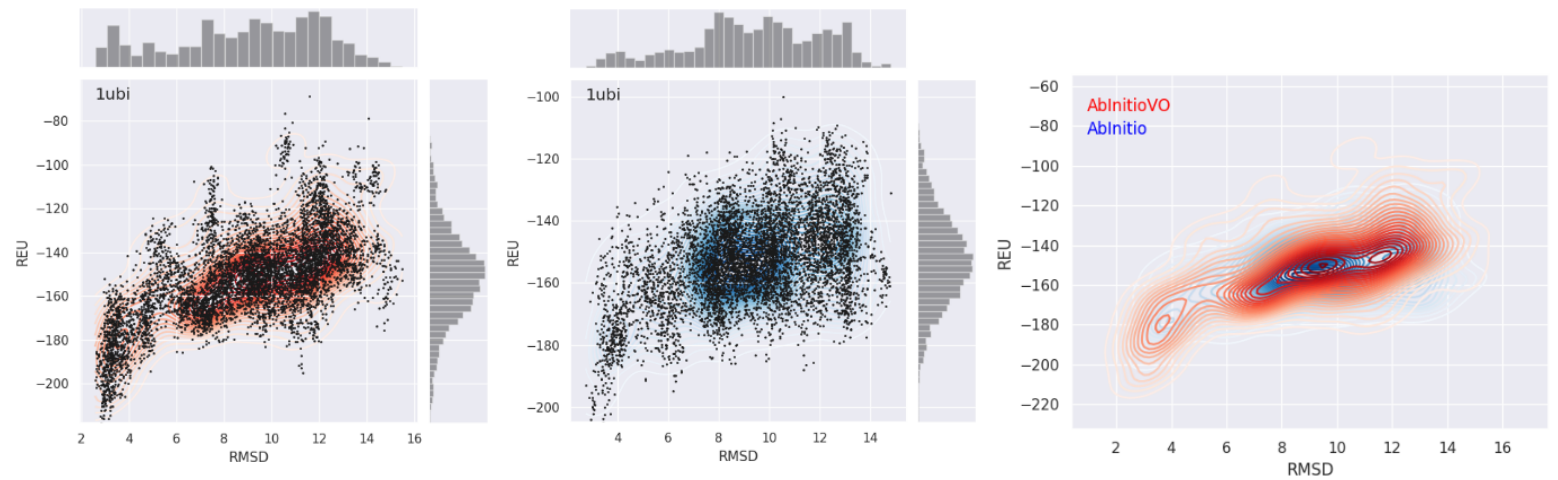

Figure S115: Folding Funnel Comparison for 1ubi. Folding funnel from AbInitioVO (left) and AbInitio (middle) simulations plotting C $\alpha$ RMSD versus Rosetta Energy Units (REU) from each structure compared to the PDB structure. For each folding funnel, histograms of the computed RMSD (top) and REU (right) are shown. KDE plot (right) showing overlay of AbInitiovo (red) and AbInitio (blue) folding funnels. 

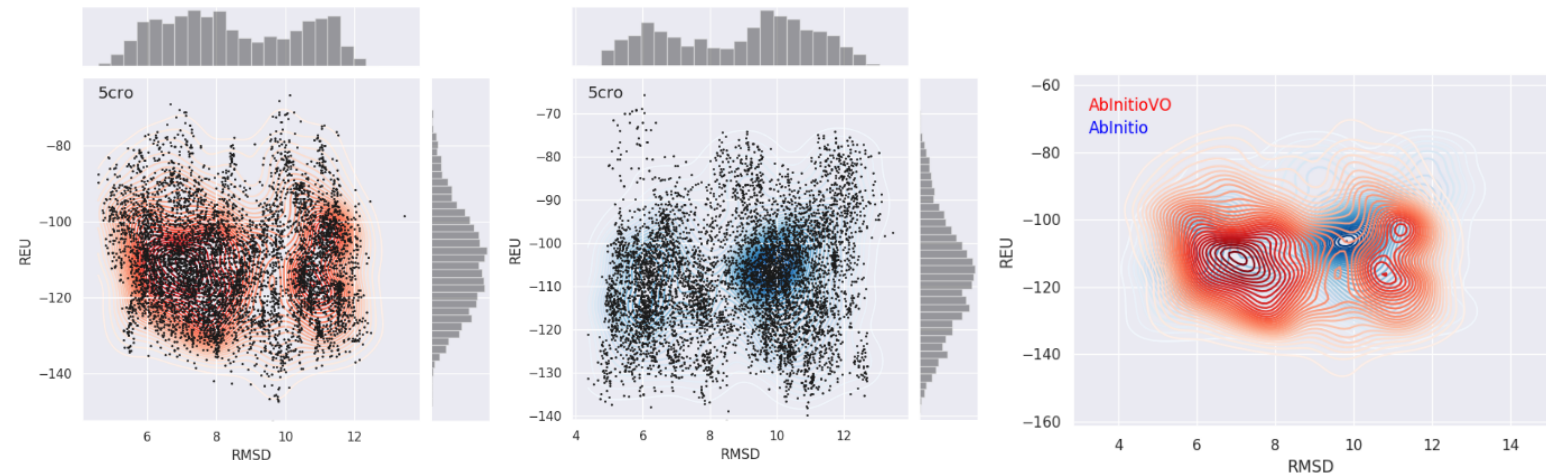

Figure S116: Folding Funnel Comparison for 5cro. Folding funnel from AbInitioVO (left) and AbInitio (middle) simulations plotting C $\alpha$ RMSD versus Rosetta Energy Units (REU) from each structure compared to the PDB structure. For each folding funnel, histograms of the computed RMSD (top) and REU (right) are shown. KDE plot (right) showing overlay of AbInitiovo (red) and AbInitio (blue) folding funnels.
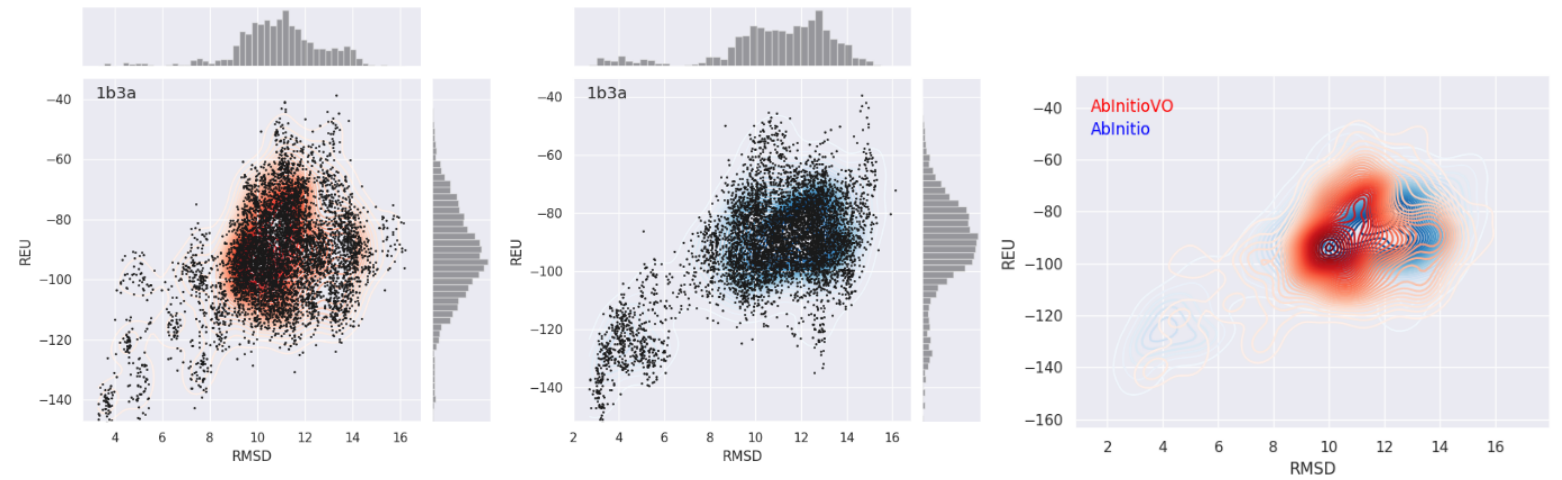

Figure S117: Folding Funnel Comparison for 1b3a. Folding funnel from AbInitioVO (left) and AbInitio (middle) simulations plotting C $\alpha$ RMSD versus Rosetta Energy Units (REU) from each structure compared to the folded domain of the PDB structure. For each folding funnel, histograms of the computed RMSD (top) and REU (right) are shown. KDE plot (right) showing overlay of AbInitiovo (red) and AbInitio (blue) folding funnels. 

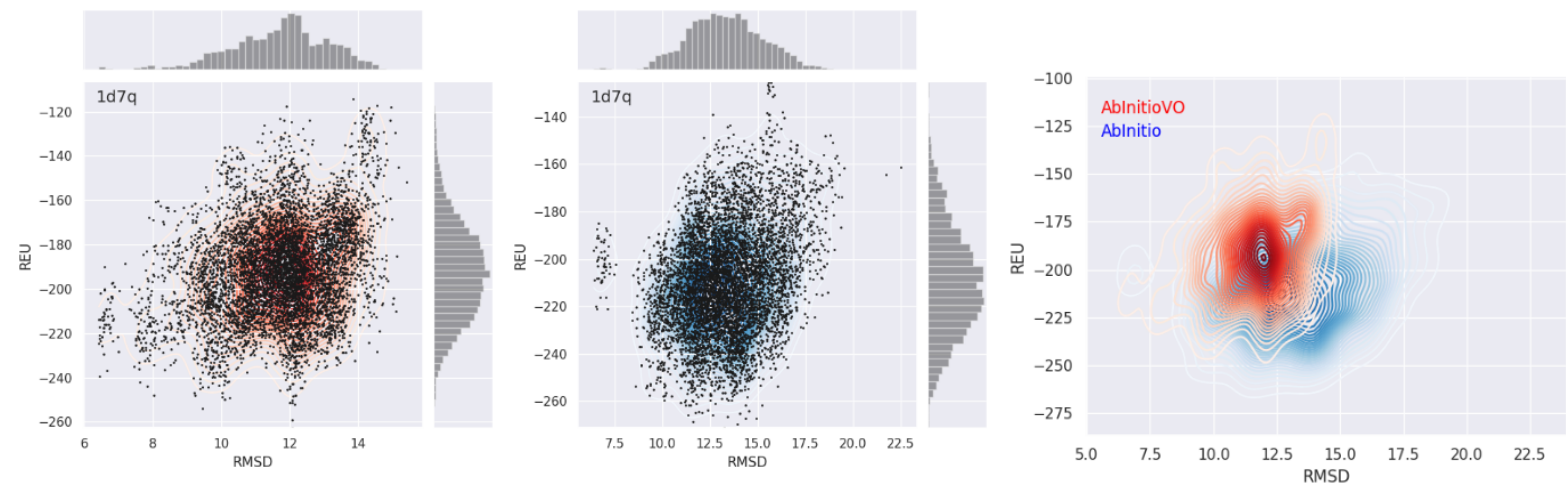

Figure S118: Folding Funnel Comparison for 1d7q. Folding funnel from AbInitioVO (left) and AbInitio (middle) simulations plotting C $\alpha$ RMSD versus Rosetta Energy Units (REU) from each structure compared to the folded domain of the PDB structure. For each folding funnel, histograms of the computed RMSD (top) and REU (right) are shown. KDE plot (right) showing overlay of AbInitiovo (red) and AbInitio (blue) folding funnels.
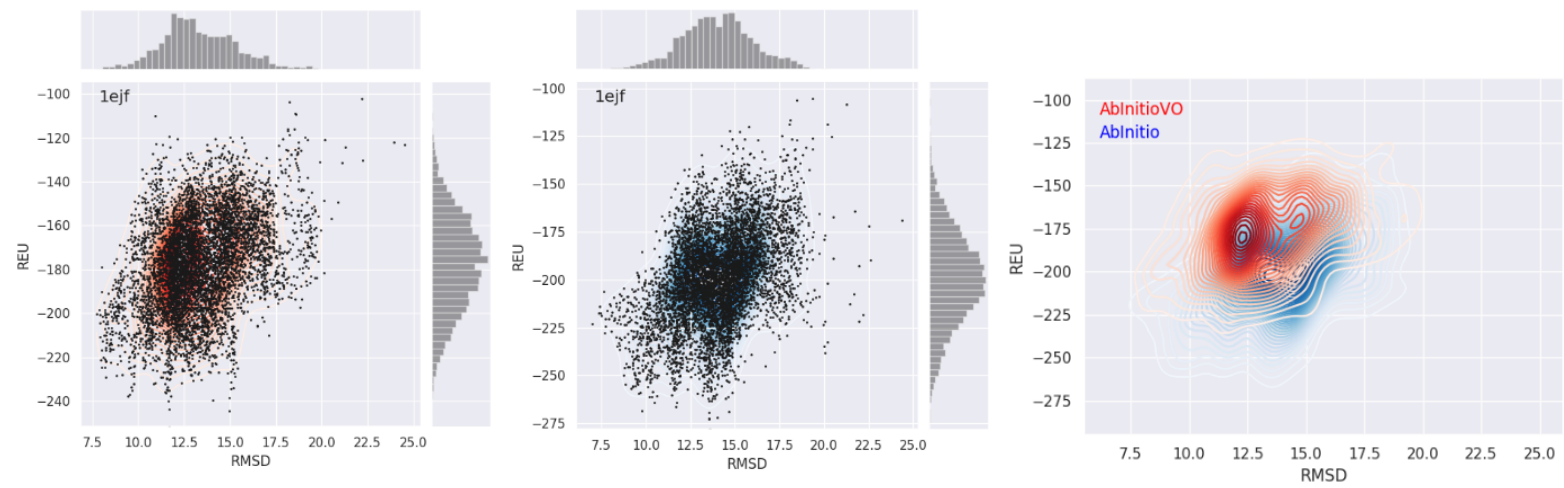

Figure S119: Folding Funnel Comparison for 1ejf. Folding funnel from AbInitioVO (left) and AbInitio (middle) simulations plotting C $\alpha$ RMSD versus Rosetta Energy Units (REU) from each structure compared to the folded domain of the PDB structure. For each folding funnel, histograms of the computed RMSD (top) and REU (right) are shown. KDE plot (right) showing overlay of AbInitiovo (red) and AbInitio (blue) folding funnels. 

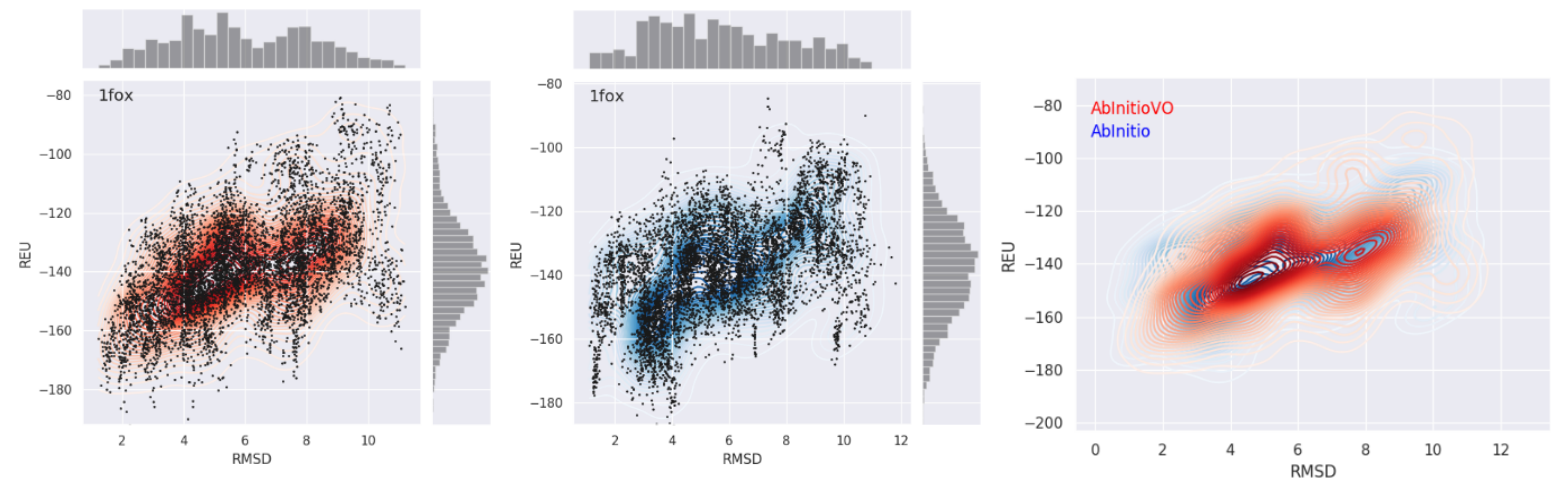

Figure S120: Folding Funnel Comparison for 1 fox. Folding funnel from AbInitioVO (left) and AbInitio (middle) simulations plotting C $\alpha$ RMSD versus Rosetta Energy Units (REU) from each structure compared to the folded domain of the PDB structure. For each folding funnel, histograms of the computed RMSD (top) and REU (right) are shown. KDE plot (right) showing overlay of AbInitiovo (red) and AbInitio (blue) folding funnels.
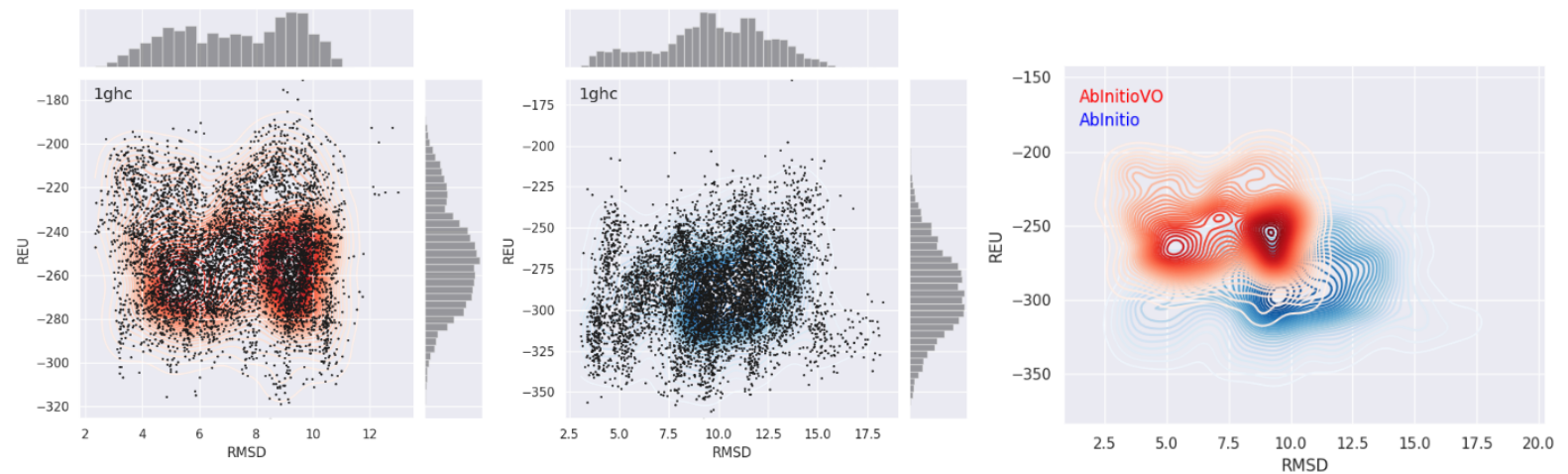

Figure S121: Folding Funnel Comparison for 1ghc. Folding funnel from AbInitioVO (left) and AbInitio (middle) simulations plotting C $\alpha$ RMSD versus Rosetta Energy Units (REU) from each structure compared to the folded domain of the PDB structure. For each folding funnel, histograms of the computed RMSD (top) and REU (right) are shown. KDE plot (right) showing overlay of AbInitiovo (red) and AbInitio (blue) folding funnels. 

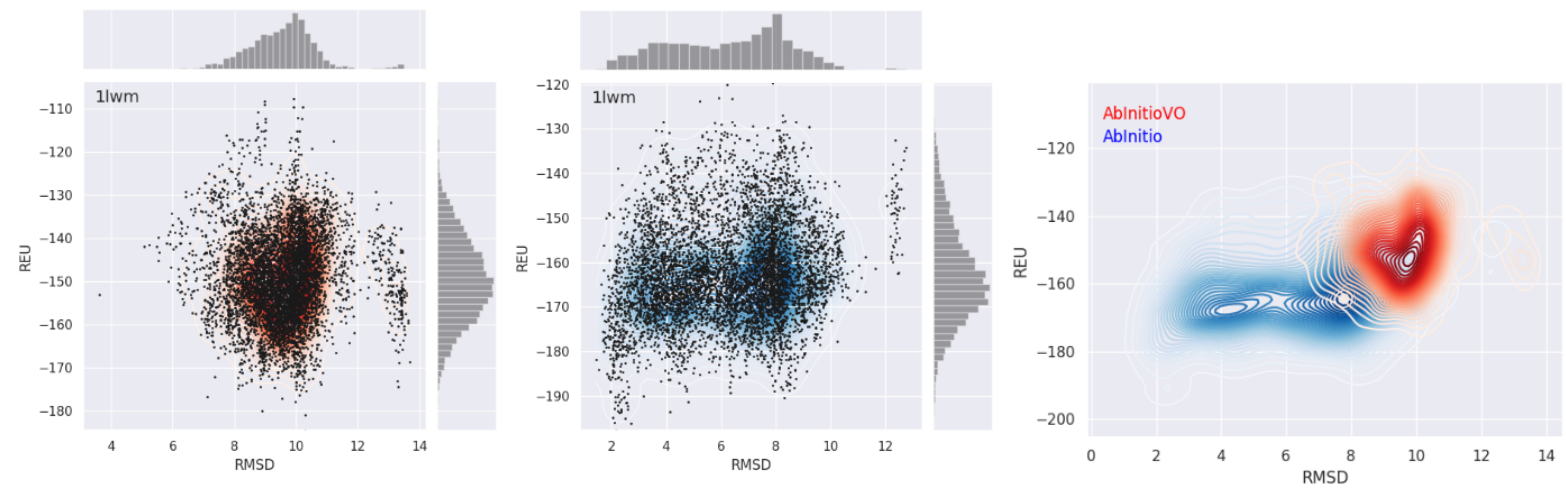

Figure S122: Folding Funnel Comparison for 1lwm. Folding funnel from AbInitioVO (left) and AbInitio (middle) simulations plotting C $\alpha$ RMSD versus Rosetta Energy Units (REU) from each structure compared to the folded domain of the PDB structure. For each folding funnel, histograms of the computed RMSD (top) and REU (right) are shown. KDE plot (right) showing overlay of AbInitiovo (red) and AbInitio (blue) folding funnels.
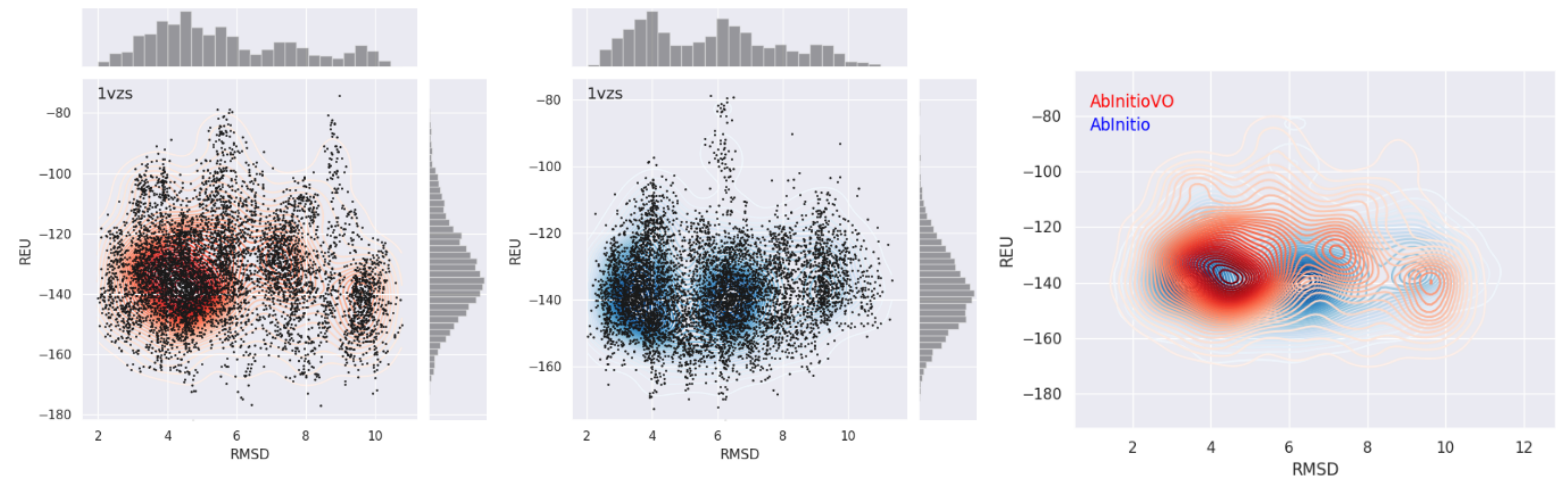

Figure S123: Folding Funnel Comparison for 1vzs. Folding funnel from AbInitioVO (left) and AbInitio (middle) simulations plotting C $\alpha$ RMSD versus Rosetta Energy Units (REU) from each structure compared to the folded domain of the PDB structure. For each folding funnel, histograms of the computed RMSD (top) and REU (right) are shown. KDE plot (right) showing overlay of AbInitiovo (red) and AbInitio (blue) folding funnels. 

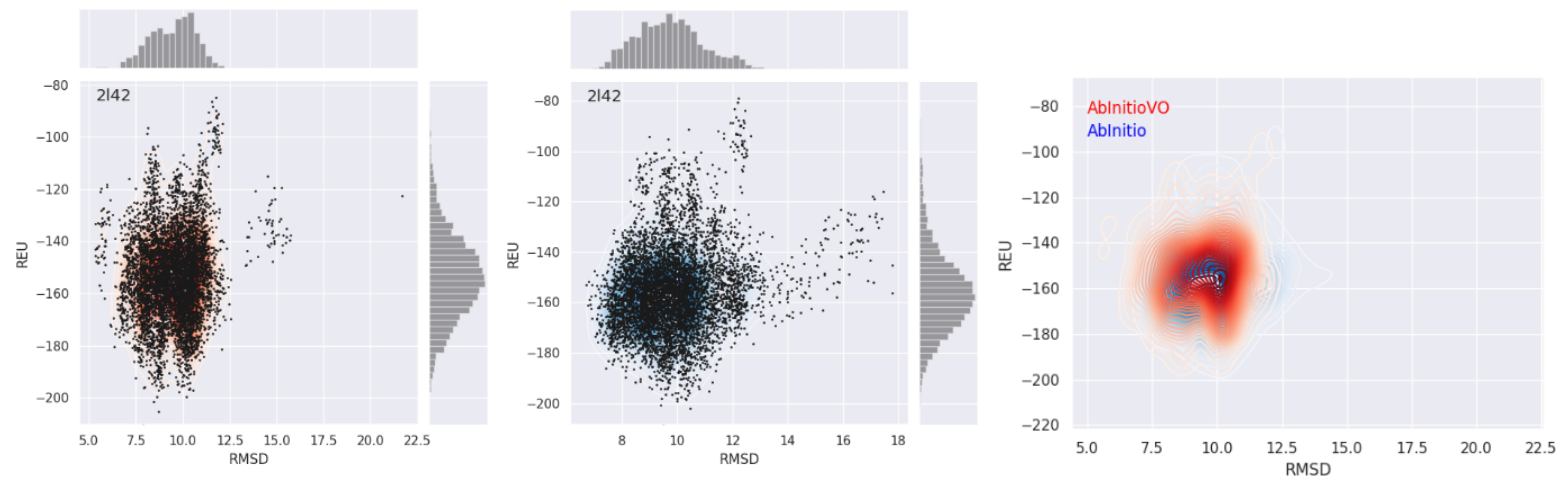

Figure S124: Folding Funnel Comparison for 2142. Folding funnel from AbInitioVO (left) and AbInitio (middle) simulations plotting C $\alpha$ RMSD versus Rosetta Energy Units (REU) from each structure compared to the folded domain of the PDB structure. For each folding funnel, histograms of the computed RMSD (top) and REU (right) are shown. KDE plot (right) showing overlay of AbInitiovo (red) and AbInitio (blue) folding funnels.
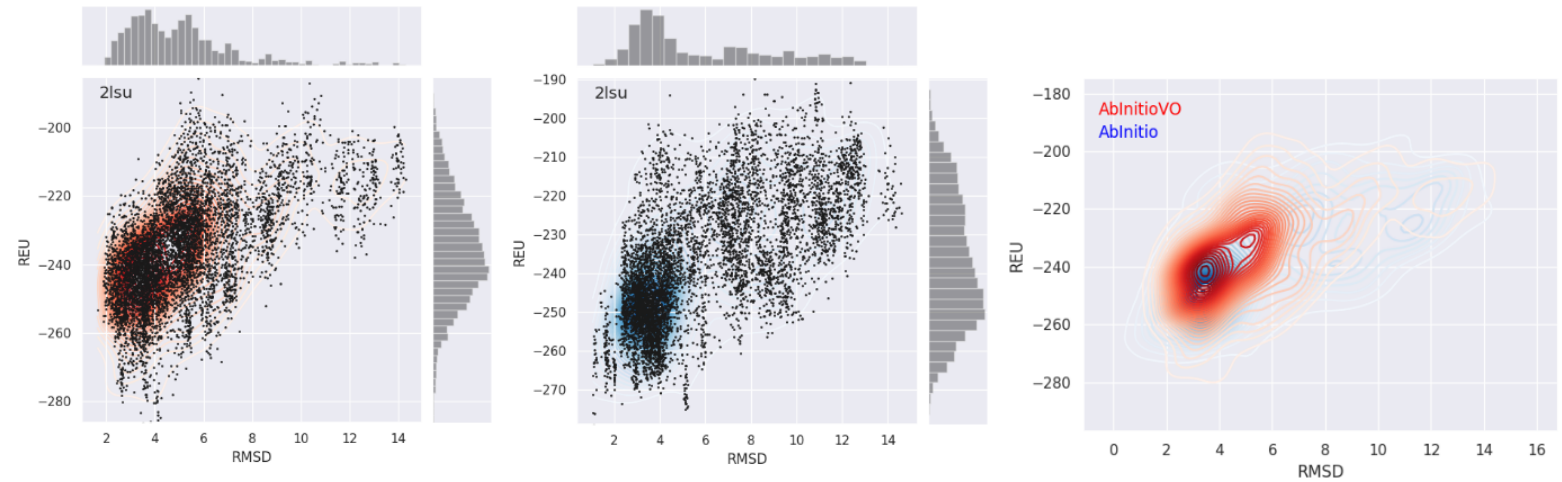

Figure S125: Folding Funnel Comparison for 2lsu. Folding funnel from AbInitioVO (left) and AbInitio (middle) simulations plotting C $\alpha$ RMSD versus Rosetta Energy Units (REU) from each structure compared to the folded domain of the PDB structure. For each folding funnel, histograms of the computed RMSD (top) and REU (right) are shown. KDE plot (right) showing overlay of AbInitiovo (red) and AbInitio (blue) folding funnels. 

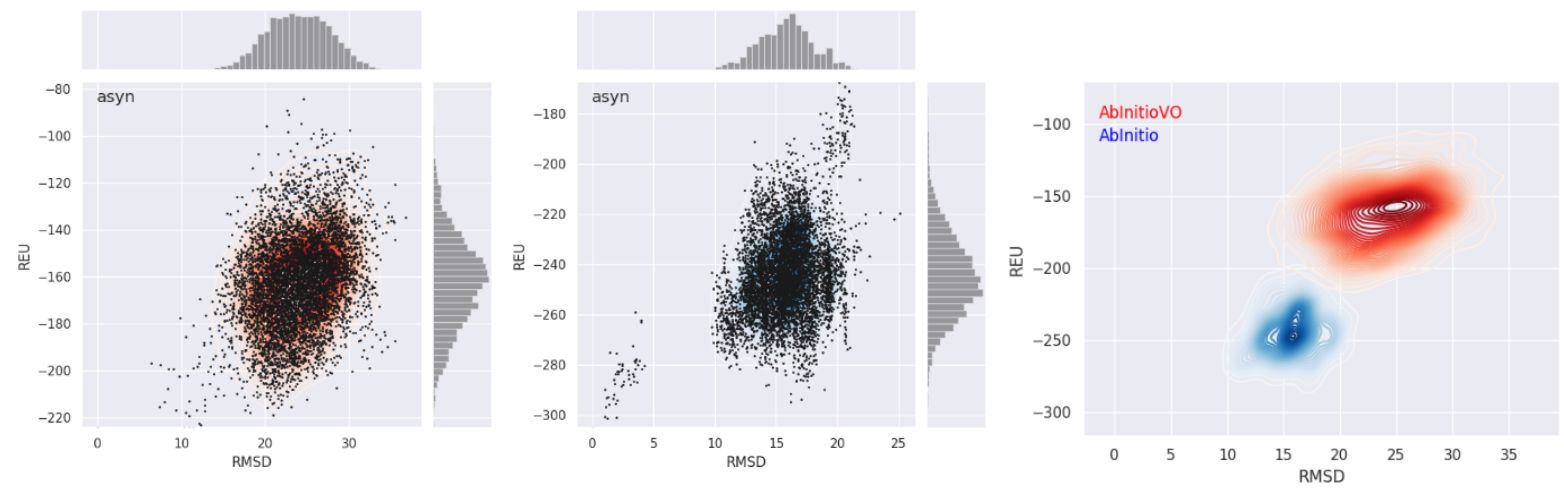

Figure S126: Folding Funnel Comparison for asyn. Folding funnel from AbInitioVO (left) and AbInitio (middle) simulations plotting C $\alpha$ RMSD versus Rosetta Energy Units (REU) from each structure compared to the lowest energy structure. For each folding funnel, histograms of the computed RMSD (top) and REU (right) are shown. KDE plot (right) showing overlay of AbInitiovo (red) and AbInitio (blue) folding funnels.
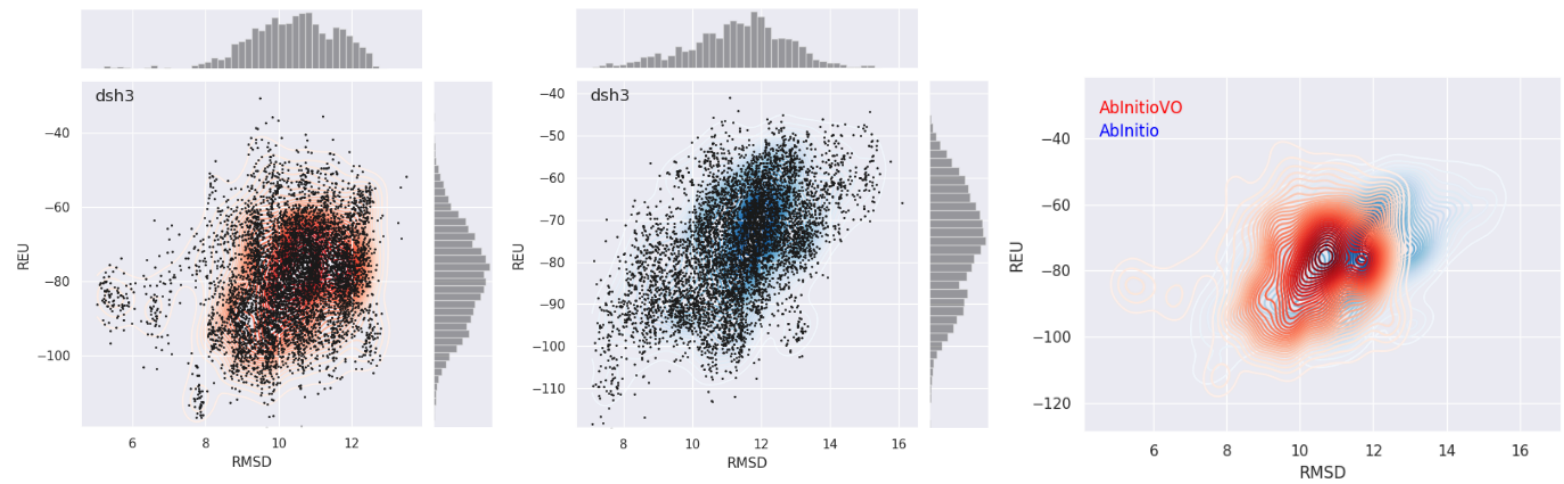

Figure S127: Folding Funnel Comparison for dsh3. Folding funnel from AbInitioVO (left) and AbInitio (middle) simulations plotting C $\alpha$ RMSD versus Rosetta Energy Units (REU) from each structure compared to the PDB structure 2A37. For each folding funnel, histograms of the computed RMSD (top) and REU (right) are shown. KDE plot (right) showing overlay of AbInitiovo (red) and AbInitio (blue) folding funnels. 

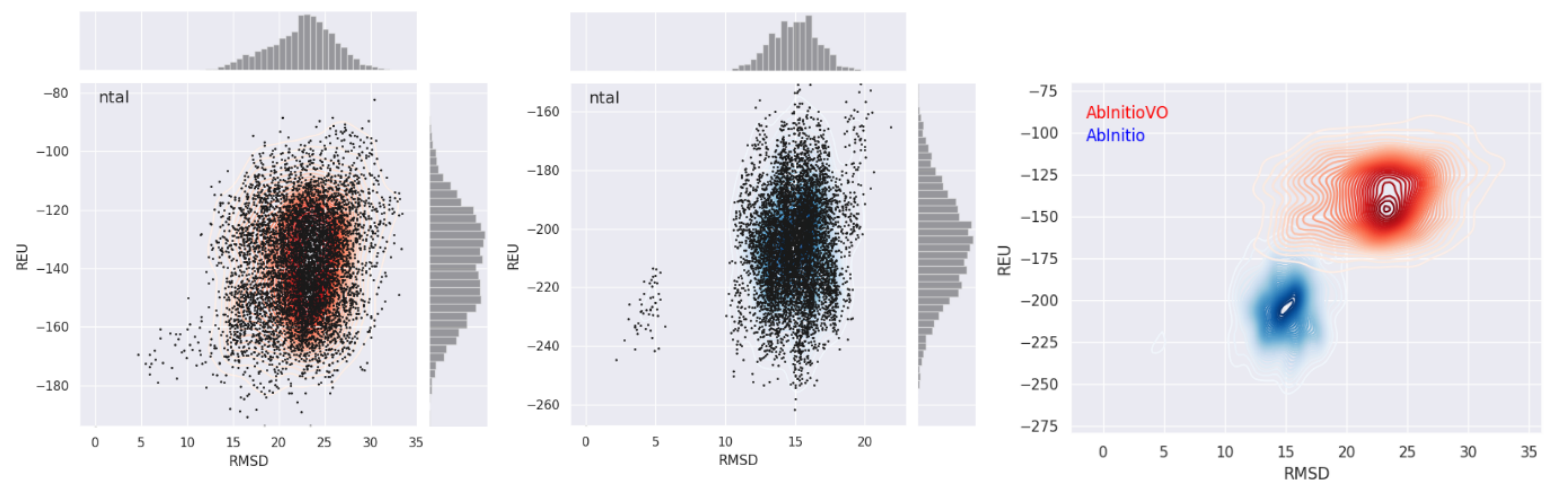

Figure S128: Folding Funnel Comparison for ntal. Folding funnel from AbInitioVO (left) and AbInitio (middle) simulations plotting C $\alpha$ RMSD versus Rosetta Energy Units (REU) from each structure compared to the lowest energy structure. For each folding funnel, histograms of the computed RMSD (top) and REU (right) are shown. KDE plot (right) showing overlay of AbInitiovo (red) and AbInitio (blue) folding funnels.
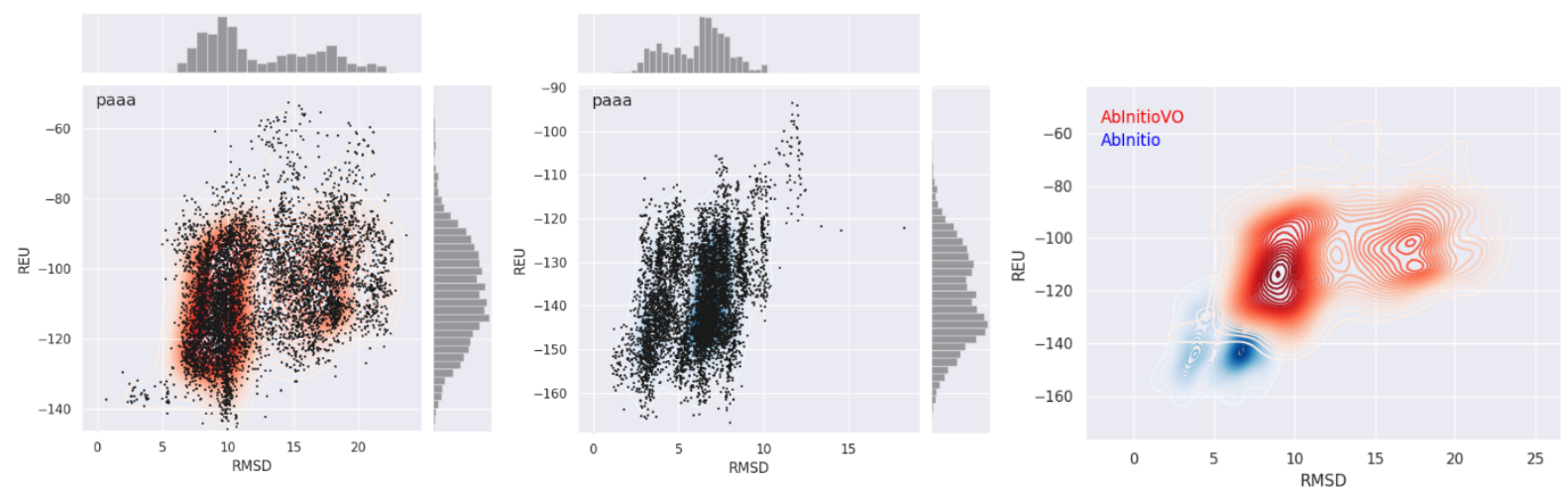

Figure S129: Folding Funnel Comparison for paaa. Folding funnel from AbInitioVO (left) and AbInitio (middle) simulations plotting C $\alpha$ RMSD versus Rosetta Energy Units (REU) from each structure compared to the lowest energy structure. For each folding funnel, histograms of the computed RMSD (top) and REU (right) are shown. KDE plot (right) showing overlay of AbInitiovo (red) and AbInitio (blue) folding funnels. 

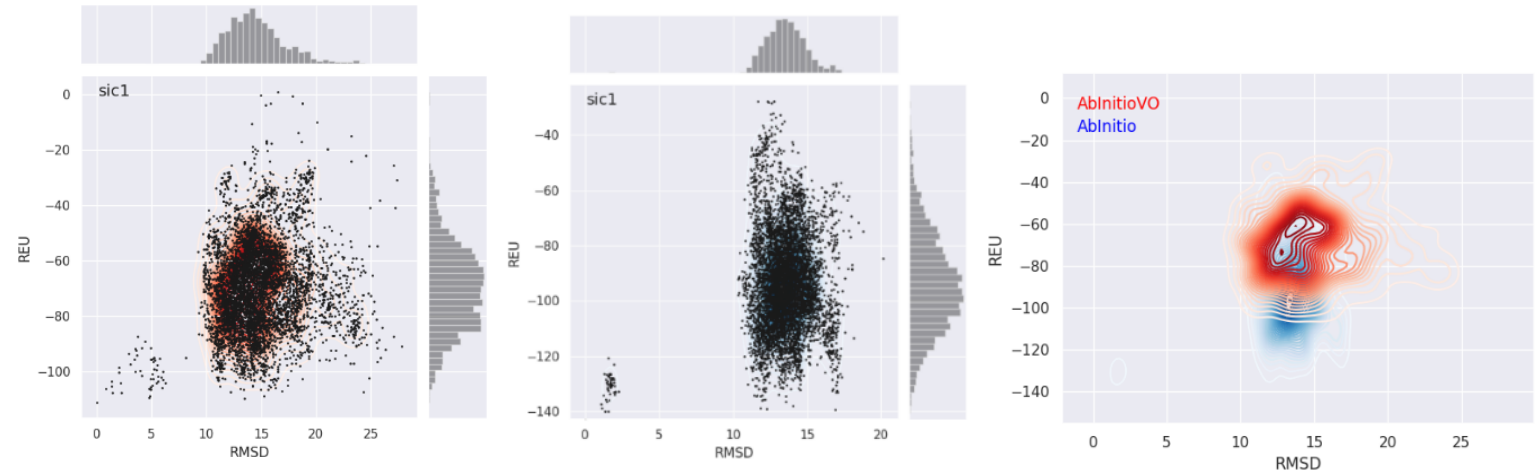

Figure S130: Folding Funnel Comparison for sic1. Folding funnel from AbInitioVO (left) and AbInitio (middle) simulations plotting C $\alpha$ RMSD versus Rosetta Energy Units (REU) from each structure compared to the lowest energy structure. For each folding funnel, histograms of the computed RMSD (top) and REU (right) are shown. KDE plot (right) showing overlay of AbInitiovo (red) and AbInitio (blue) folding funnels.
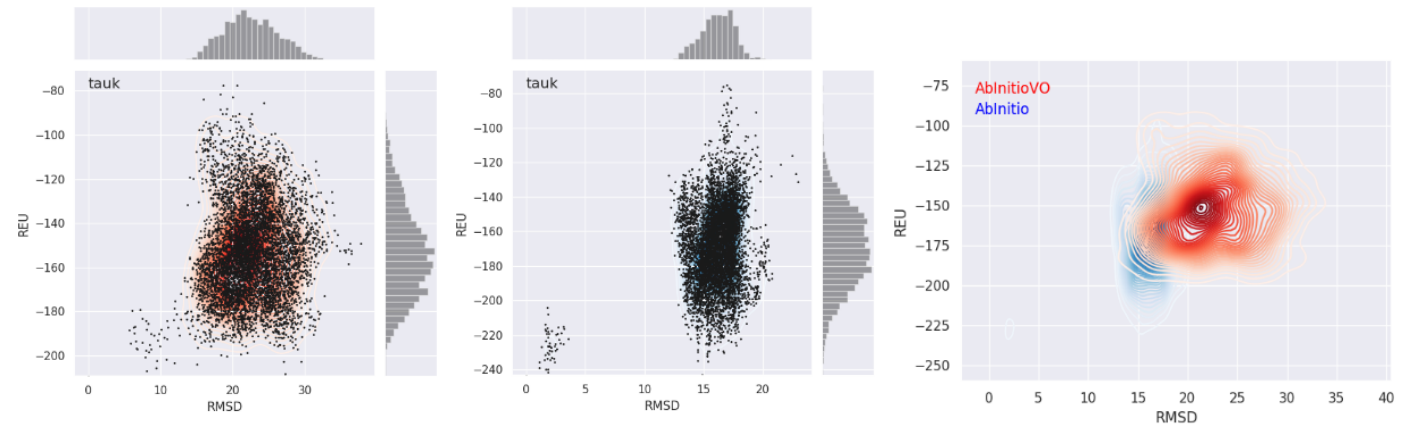

Figure S131: Folding Funnel Comparison for tauk. Folding funnel from AbInitioVO (left) and AbInitio (middle) simulations plotting C $\alpha$ RMSD versus Rosetta Energy Units (REU) from each structure compared to the lowest energy structure. For each folding funnel, histograms of the computed RMSD (top) and REU (right) are shown. KDE plot (right) showing overlay of AbInitiovo (red) and AbInitio (blue) folding funnels. 

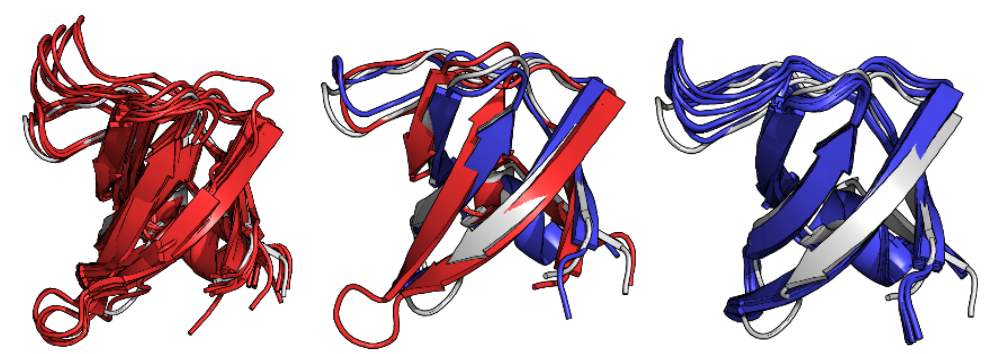

Figure S132: 1bk2 Lowest RMSD Structures. 10 lowest RMSD structures from AbInitioVO (left) and AbInitio (right) along with the overlay (center) of PDB 1bk2 (white) and the single lowest RMSD structure from AbInitioVO (red) and AbInitio (blue)
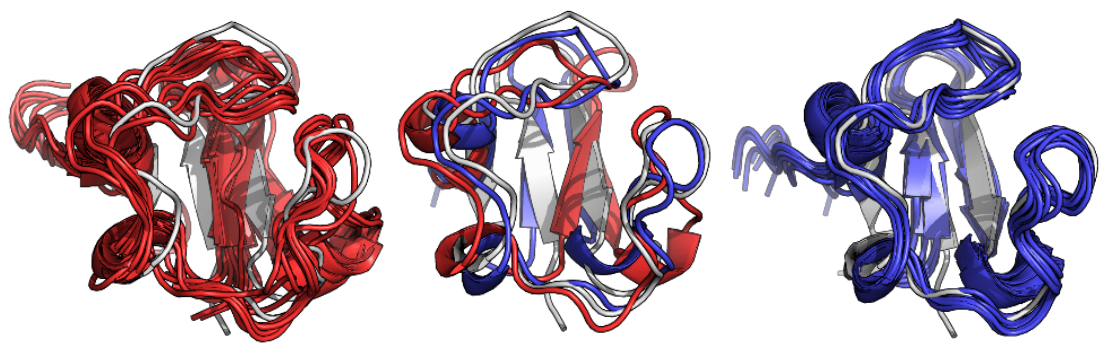

Figure S133: 1bq9 Lowest RMSD Structures. 10 lowest RMSD structures from AbInitioVO (left) and AbInitio (right) along with the overlay (center) of PDB 1bq9 (white) and the single lowest RMSD structure from AbInitioVO (red) and AbInitio (blue)
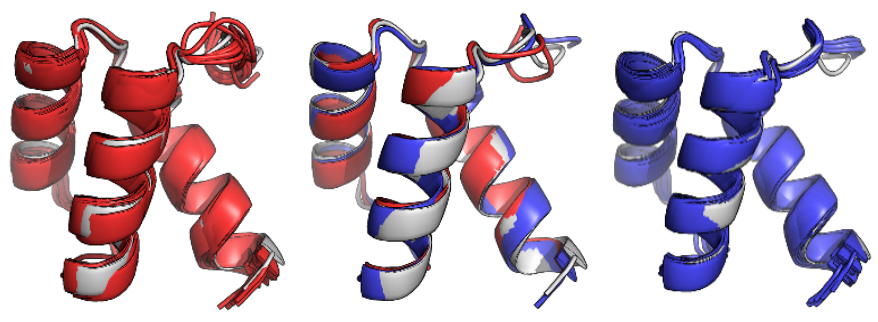

Figure S134: 1enh Lowest RMSD Structures. 10 lowest RMSD structures from AbInitioVO (left) and AbInitio (right) along with the overlay (center) of PDB 1enh (white) and the single lowest RMSD structure from AbInitioVO (red) and AbInitio (blue) 

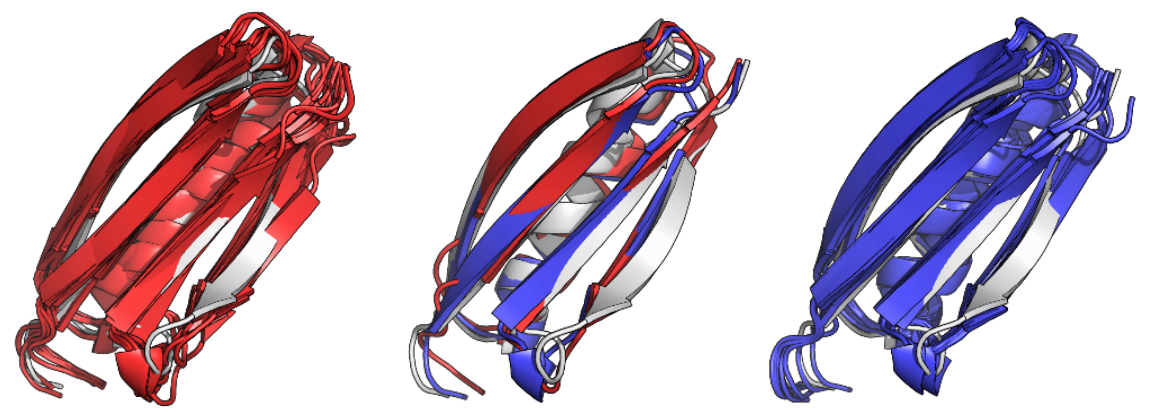

Figure S135: $1 \mathrm{hz} 6$ Lowest RMSD Structures. 10 lowest RMSD structures from AbInitioVO (left) and AbInitio (right) along with the overlay (center) of PDB 1hz6 (white) and the single lowest RMSD structure from AbInitioVO (red) and AbInitio (blue)
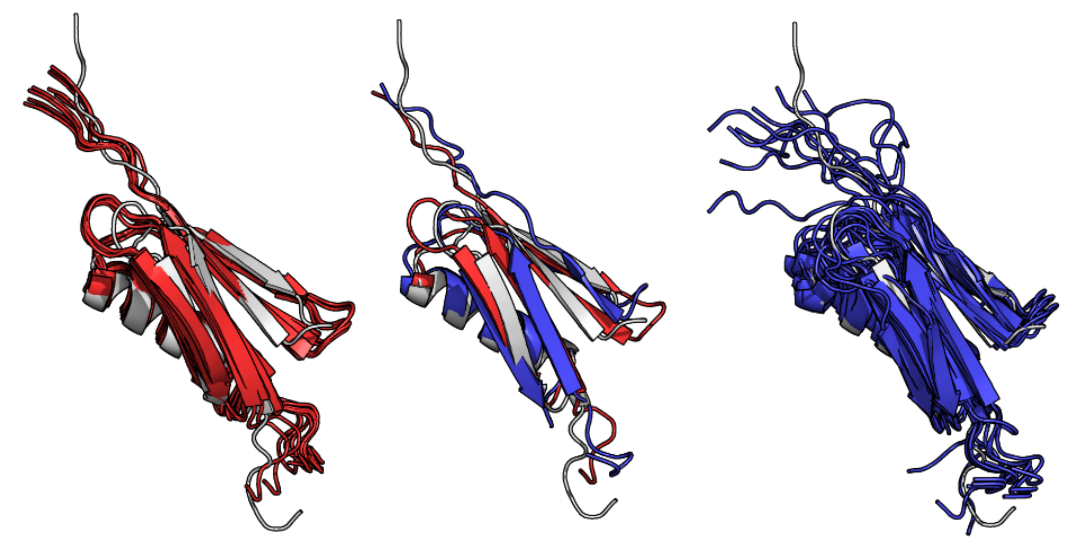

Figure S136: 1 pgx Lowest RMSD Structures. 10 lowest RMSD structures from AbInitioVO (left) and AbInitio (right) along with the overlay (center) of PDB 1pgx (white) and the single lowest RMSD structure from AbInitioVO (red) and AbInitio (blue)
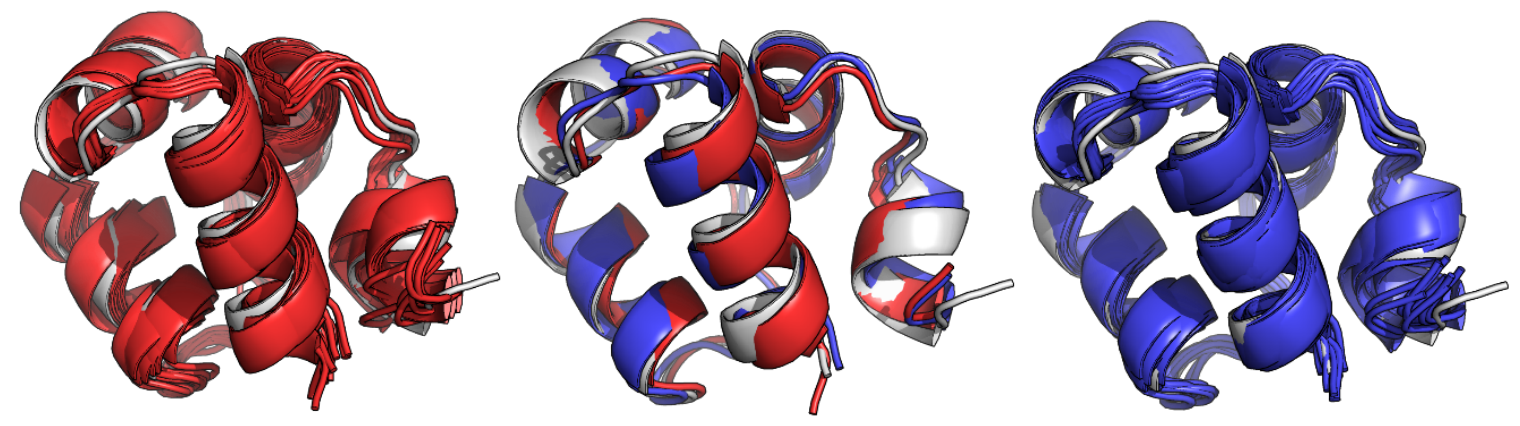

Figure S137: 1r69 Lowest RMSD Structures. 10 lowest RMSD structures from AbInitioVO (left) and AbInitio (right) along with the overlay (center) of PDB 1r69 (white) and the single lowest RMSD structure from AbInitioVO (red) and AbInitio (blue) 

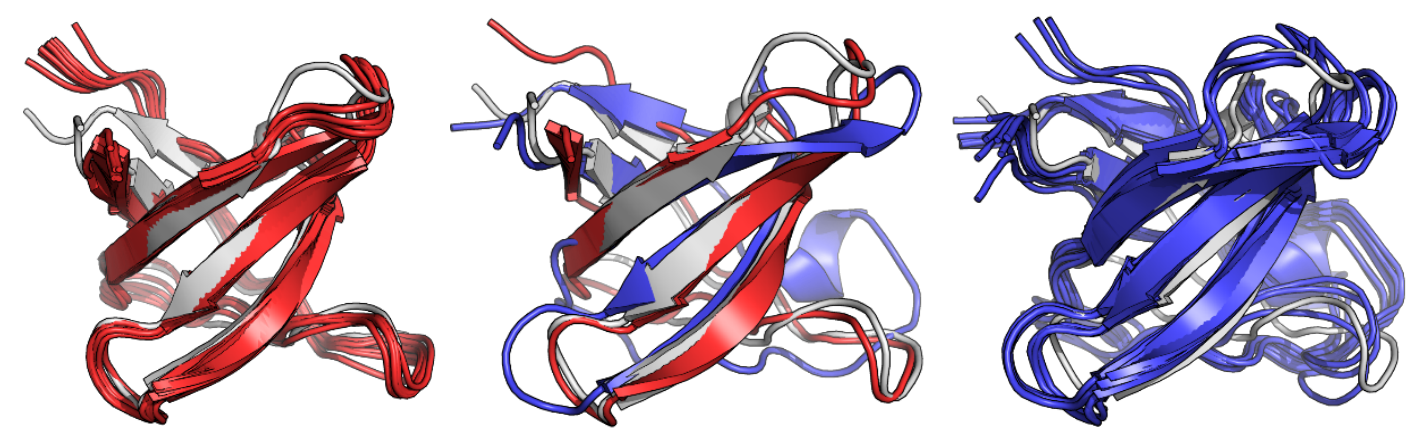

Figure S138: 1shf Lowest RMSD Structures. 10 lowest RMSD structures from AbInitioVO (left) and AbInitio (right) along with the overlay (center) of PDB 1shf (white) and the single lowest RMSD structure from AbInitioVO (red) and AbInitio (blue)
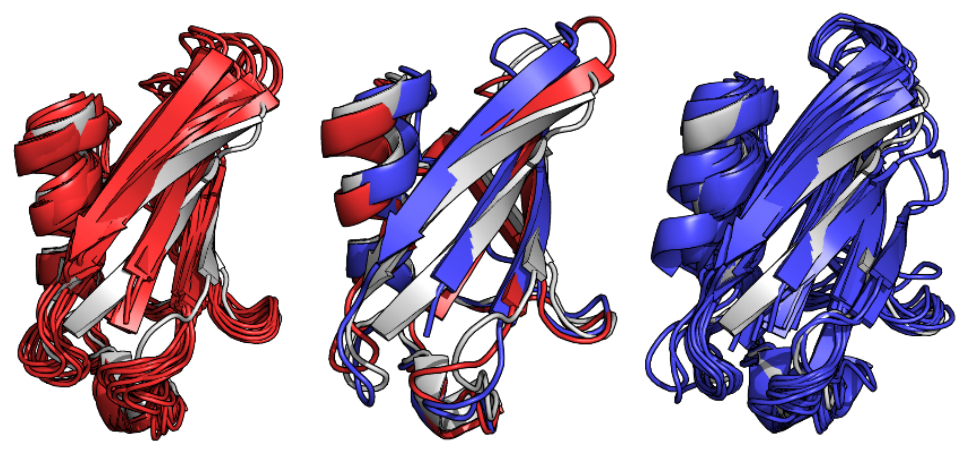

Figure S139: 1ubi Lowest RMSD Structures. 10 lowest RMSD structures from AbInitioVO (left) and AbInitio (right) along with the overlay (center) of PDB lubi (white) and the single lowest RMSD structure from AbInitioVO (red) and AbInitio (blue)
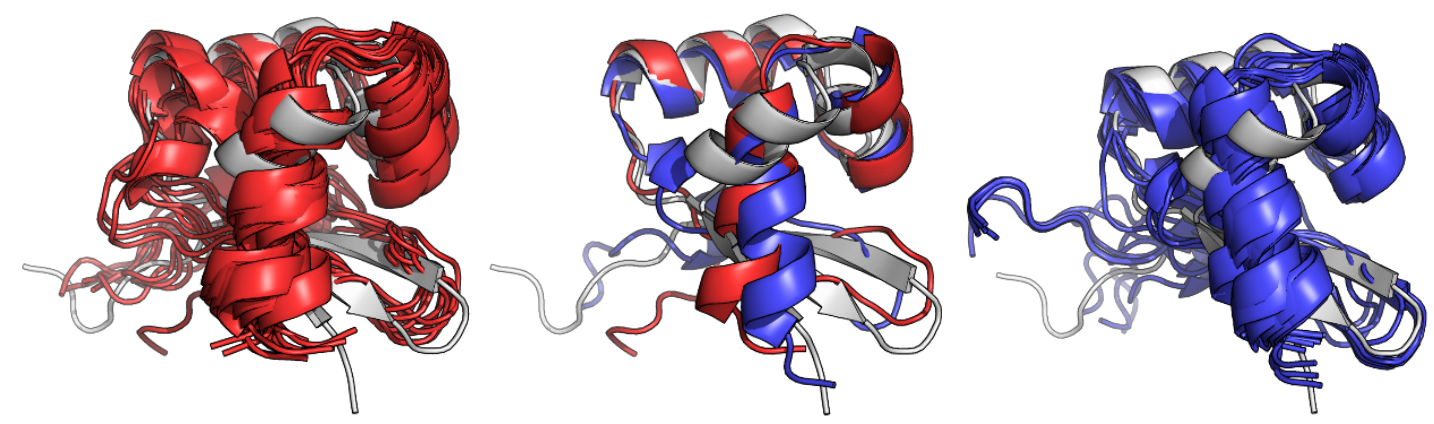

Figure S140: 5cro Lowest RMSD Structures. 10 lowest RMSD structures from AbInitioVO (left) and AbInitio (right) along with the overlay (center) of PDB 5cro (white) and the single lowest RMSD structure from AbInitioVO (red) and AbInitio (blue) 


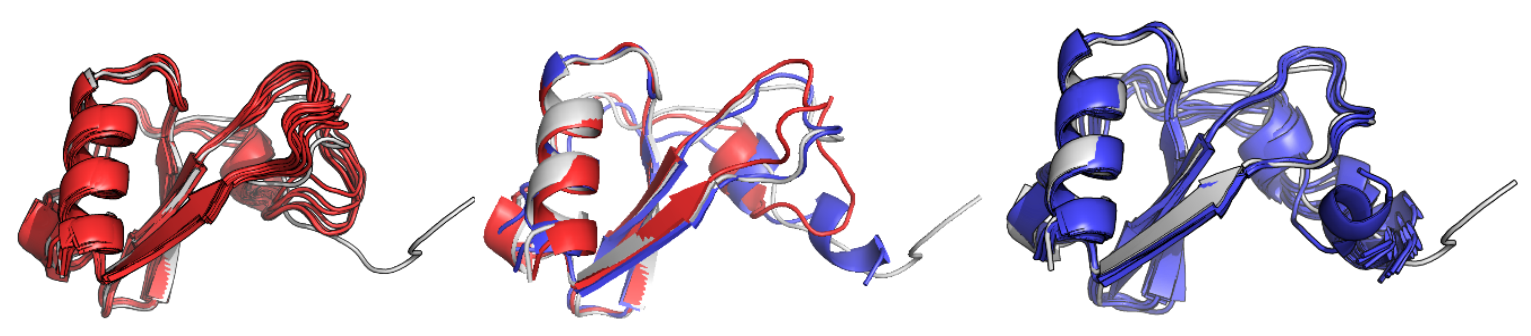

Figure S141: 1b3a Lowest RMSD Structures. 10 lowest RMSD structures from AbInitioVO (left) and AbInitio (right) along with the overlay (center) of PDB 1b3a (white) and the single lowest RMSD structure from AbInitioVO (red) and AbInitio (blue)
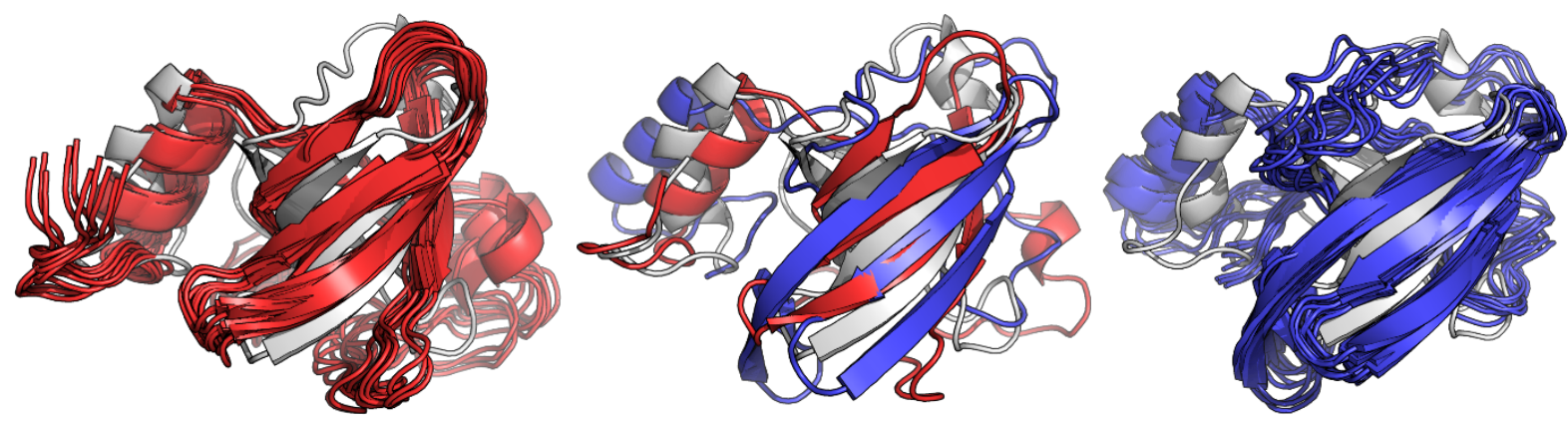

Figure S142: 1d7q Lowest RMSD Structures. 10 lowest RMSD structures from AbInitioVO (left) and AbInitio (right) along with the overlay (center) of PDB 1d7q (white) and the single lowest RMSD structure from AbInitioVO (red) and AbInitio (blue)
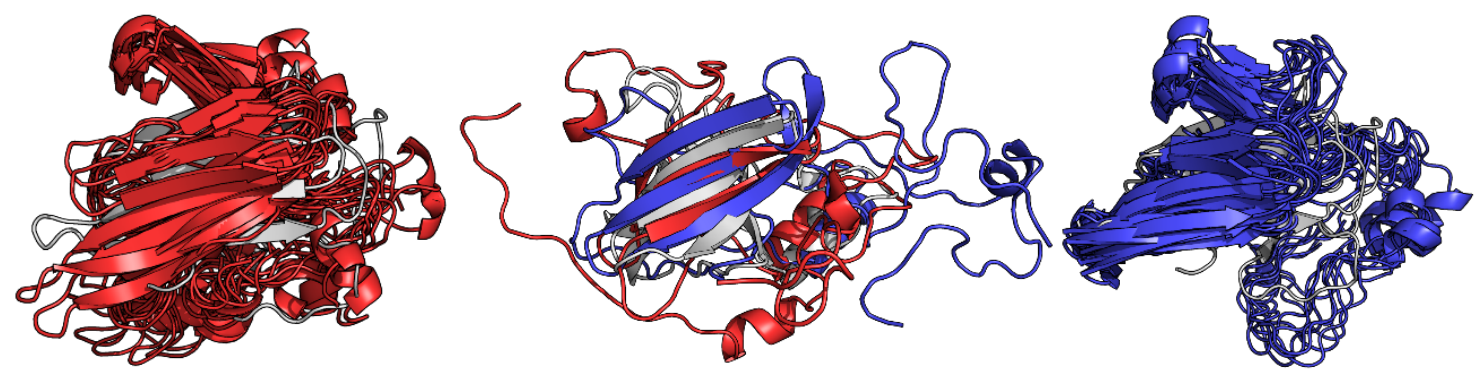

Figure S143: 1ejf Lowest RMSD Structures. 10 lowest RMSD structures from AbInitioVO (left) and AbInitio (right) along with the overlay (center) of PDB 1ejf (white) and the single lowest RMSD structure from AbInitioVO (red) and AbInitio (blue) 

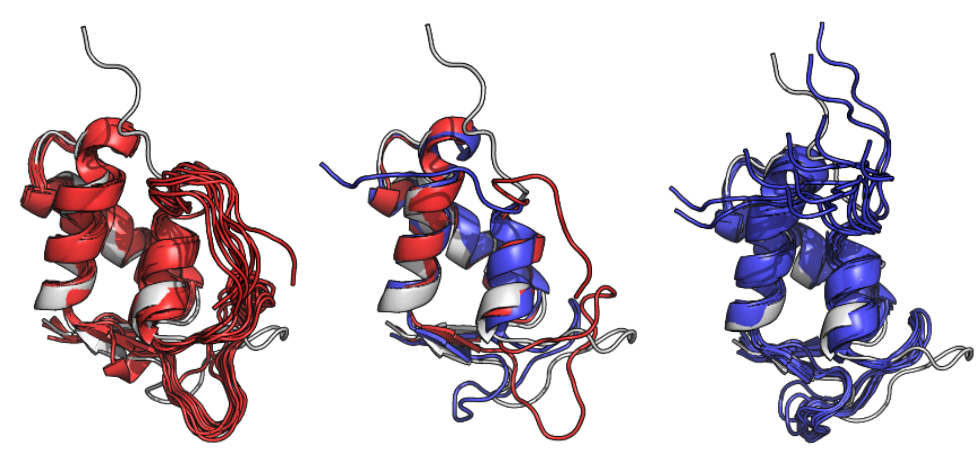

Figure S144: 1 fox Lowest RMSD Structures. 10 lowest RMSD structures from AbInitioVO (left) and AbInitio (right) along with the overlay (center) of PDB 1 fox (white) and the single lowest RMSD structure from AbInitioVO (red) and AbInitio (blue)
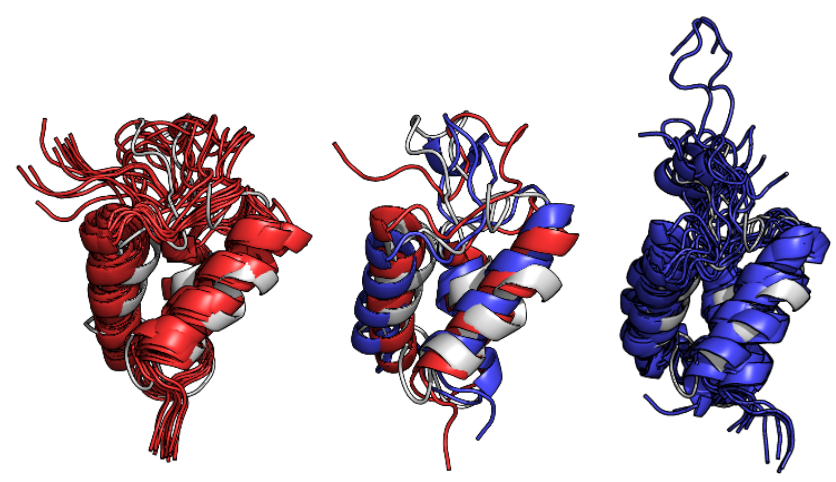

Figure S145: 1ghc Lowest RMSD Structures. 10 lowest RMSD structures from AbInitioVO (left) and AbInitio (right) along with the overlay (center) of PDB 1ghc (white) and the single lowest RMSD structure from AbInitioVO (red) and AbInitio (blue)
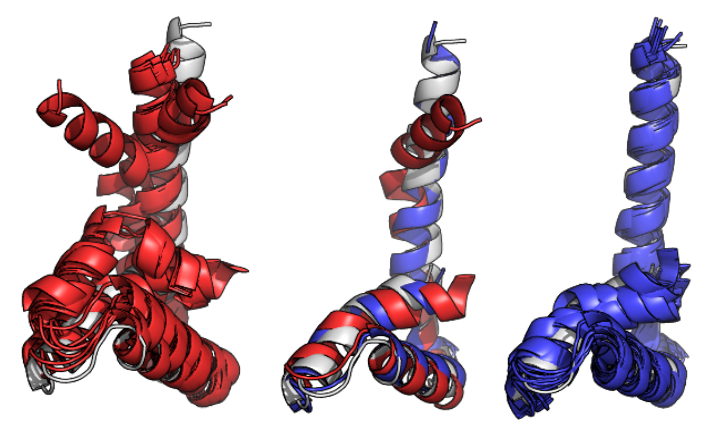

Figure S146: 1lwm Lowest RMSD Structures. 10 lowest RMSD structures from AbInitioVO (left) and AbInitio (right) along with the overlay (center) of PDB 1lwm (white) and the single lowest RMSD structure from AbInitioVO (red) and AbInitio (blue) 

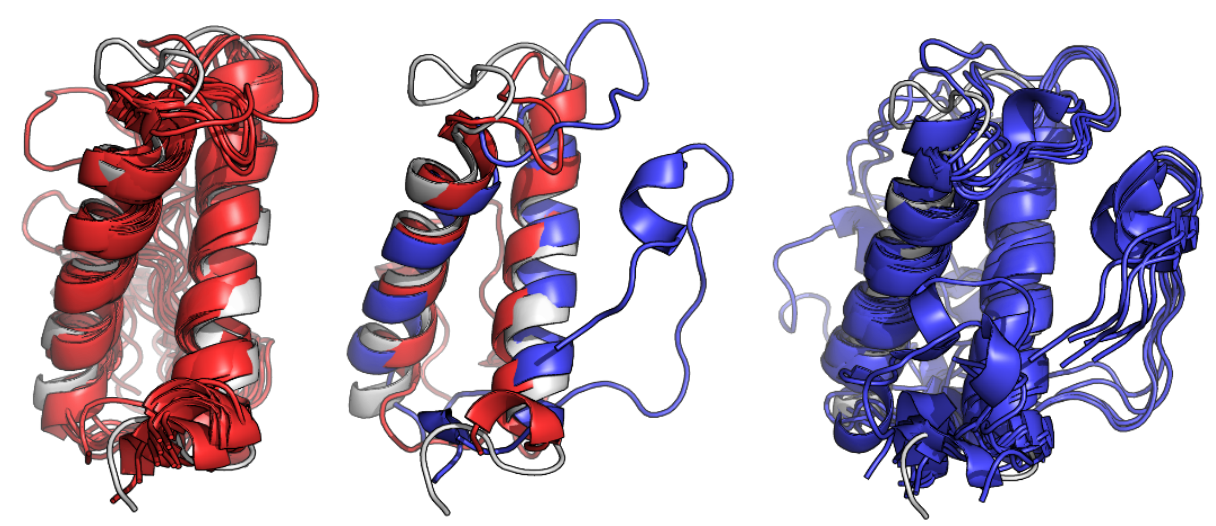

Figure S147: 1vzs Lowest RMSD Structures. 10 lowest RMSD structures from AbInitioVO (left) and AbInitio (right) along with the overlay (center) of PDB 1vzs (white) and the single lowest RMSD structure from AbInitioVO (red) and AbInitio (blue)
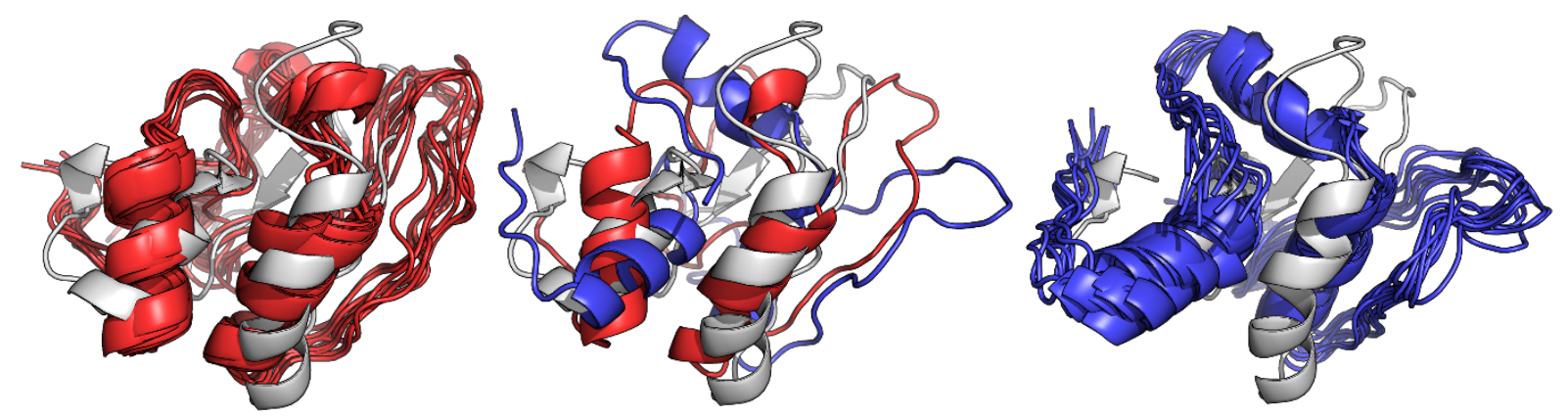

Figure S148: 2142 Lowest RMSD Structures. 10 lowest RMSD structures from AbInitioVO (left) and AbInitio (right) along with the overlay (center) of PDB 2142 (white) and the single lowest RMSD structure from AbInitioVO (red) and AbInitio (blue)
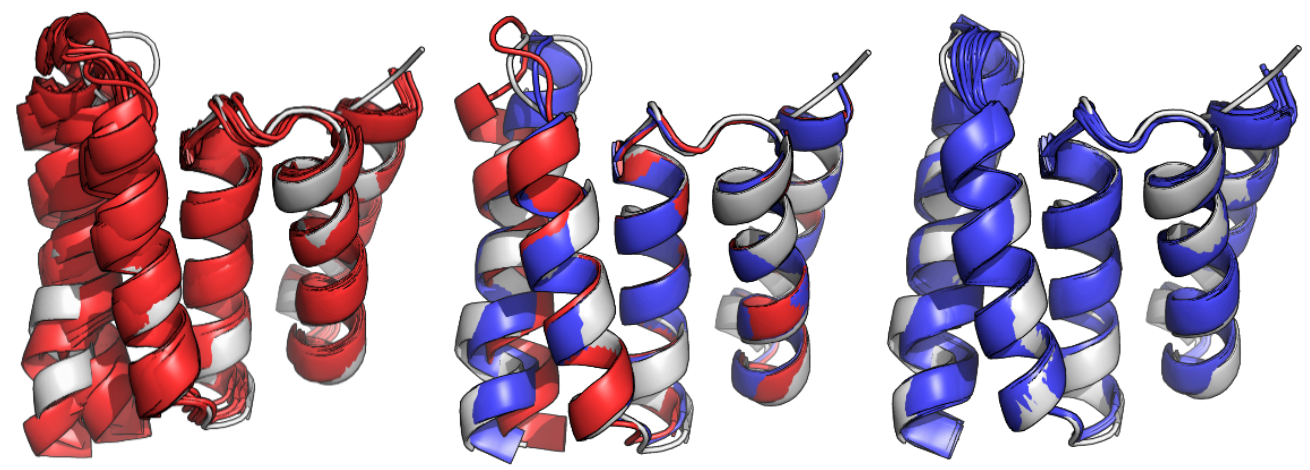

Figure S149: 21su Lowest RMSD Structures. 10 lowest RMSD structures from AbInitioVO (left) and AbInitio (right) along with the overlay (center) of PDB 2lsu (white) and the single lowest RMSD structure from AbInitioVO (red) and AbInitio (blue) 

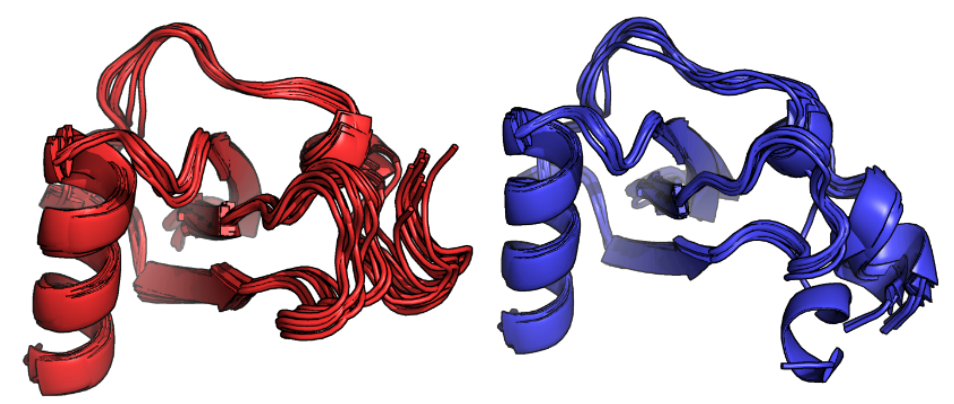

Figure S150: 1b3a Lowest RMSD Full Structures. 10 lowest RMSD structures from AbInitioVO (left) and AbInitio (right)
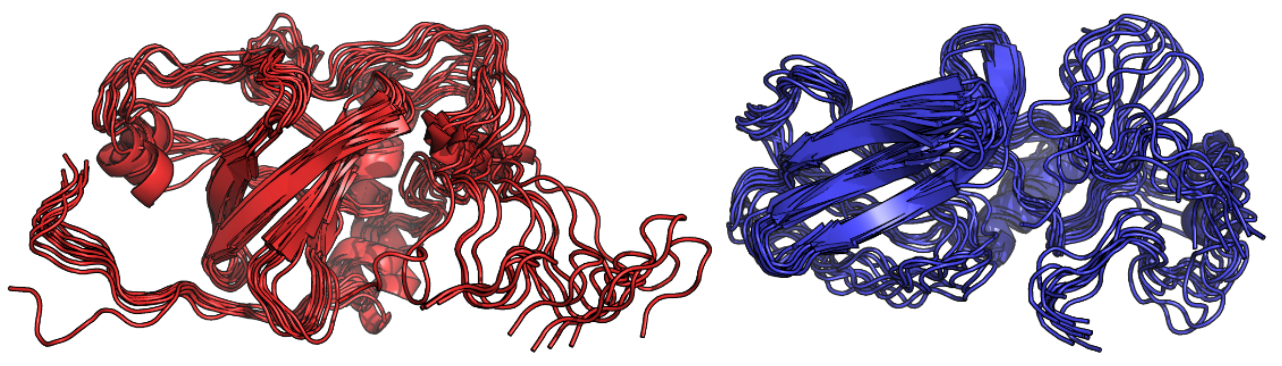

Figure S151: 1d7q Lowest RMSD Full Structures. 10 lowest RMSD structures from AbInitioVO (left) and AbInitio (right)

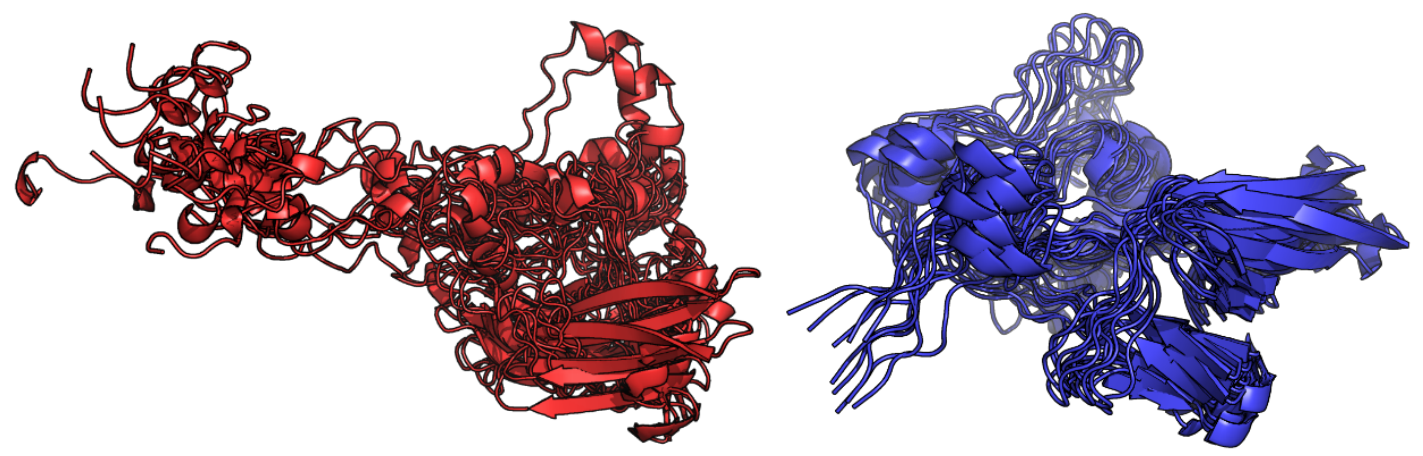

Figure S152: 1 ejf Lowest RMSD Full Structures. 10 lowest RMSD structures from AbInitioVO (left) and AbInitio (right) 

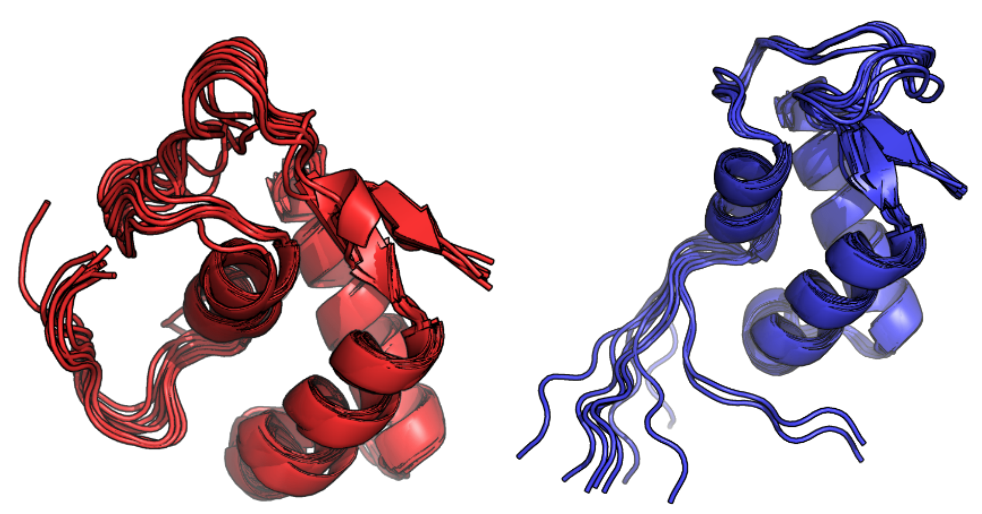

Figure S153: 1 fox Lowest RMSD Full Structures. 10 lowest RMSD structures from AbInitioVO (left) and AbInitio (right)
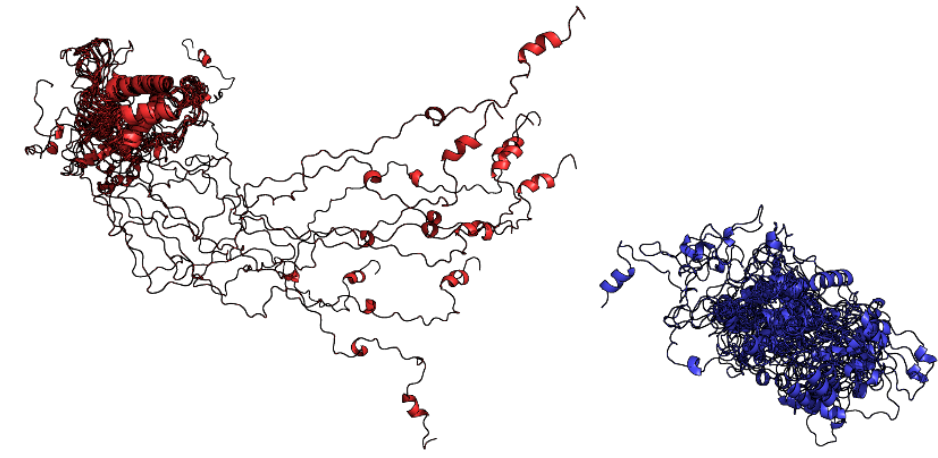

Figure S154: 1 ghc Lowest RMSD Full Structures. 10 lowest RMSD structures from AbInitioVO (left) and AbInitio (right)
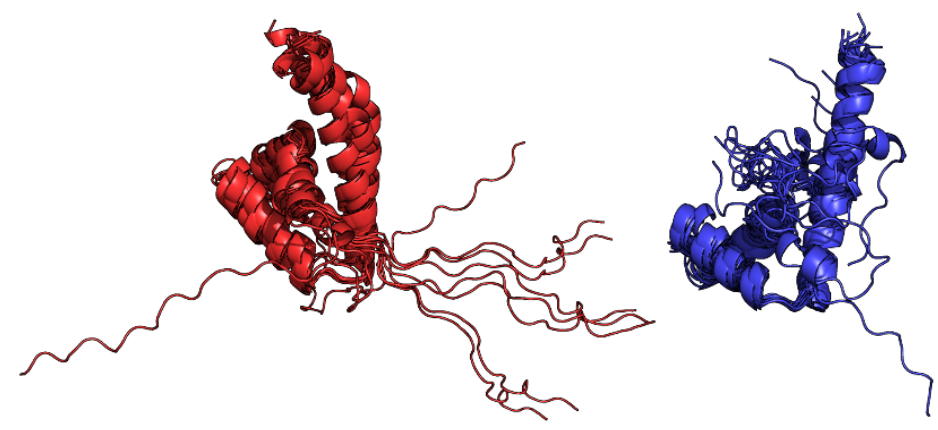

Figure S155: 11wm Lowest RMSD Full Structures. 10 lowest RMSD structures from AbInitioVO (left) and AbInitio (right) 

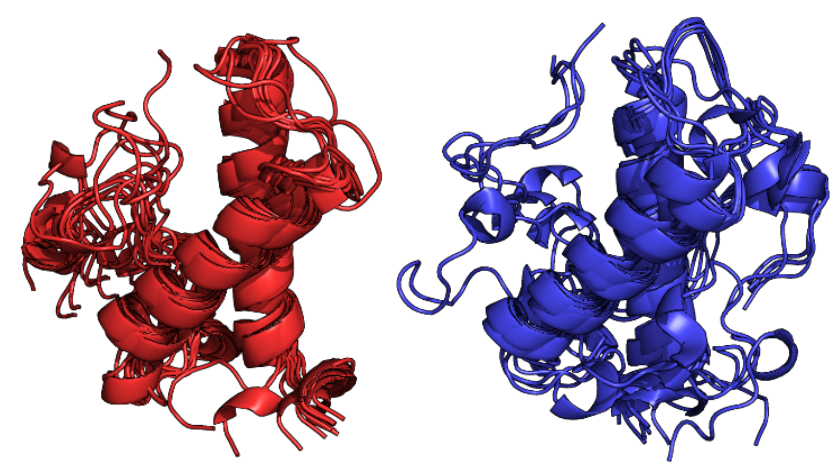

Figure S156: 1vzs Lowest RMSD Full Structures. 10 lowest RMSD structures from AbInitioVO (left) and AbInitio (right)
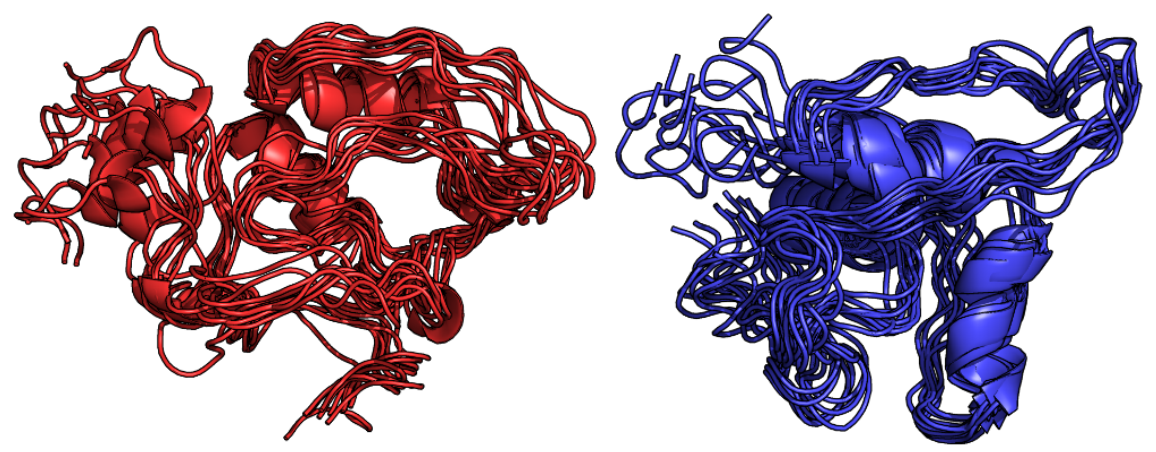

Figure S157: 2142 Lowest RMSD Full Structures. 10 lowest RMSD structures from AbInitioVO (left) and AbInitio (right)

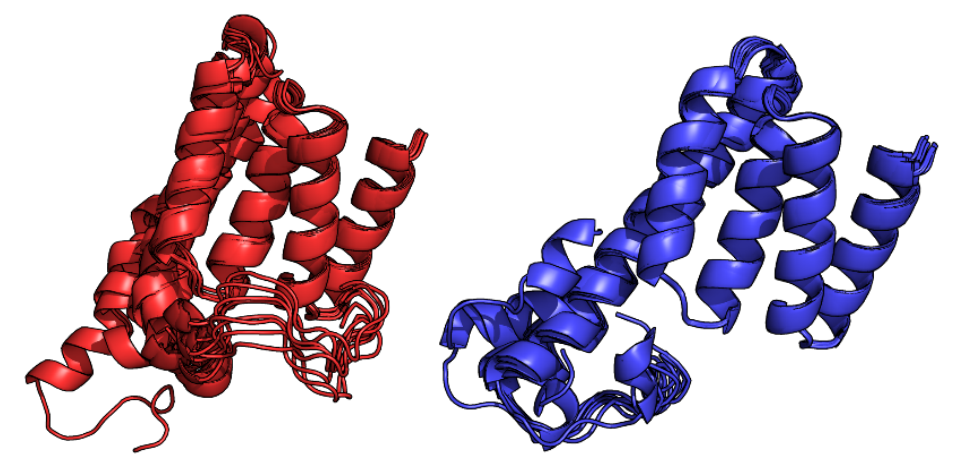

Figure S158: 21su Lowest RMSD Full Structures. 10 lowest RMSD structures from AbInitioVO (left) and AbInitio (right) 

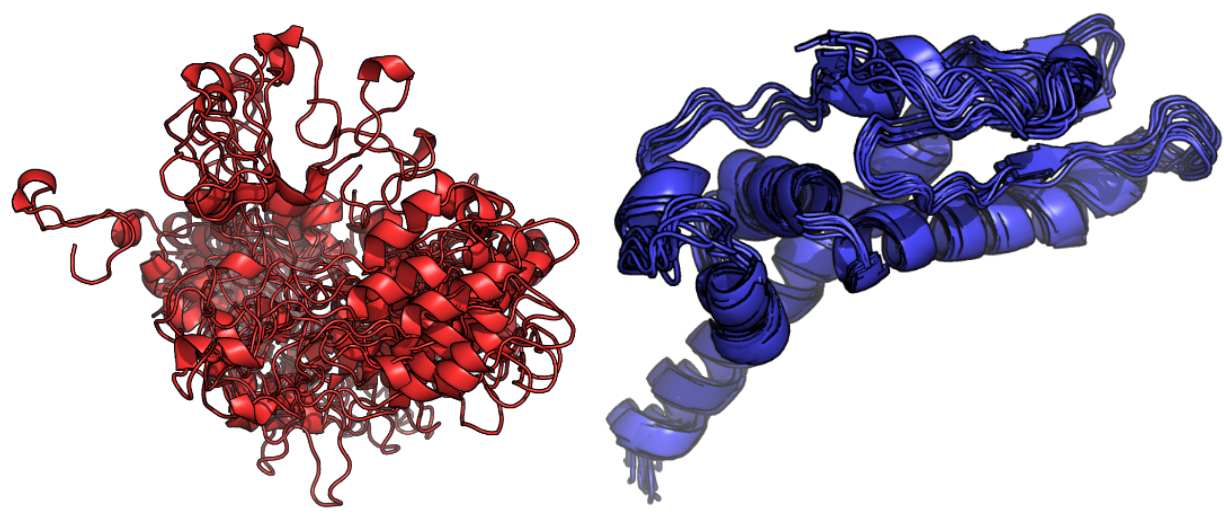

Figure S159: Asyn Lowest RMSD Full Structures. 10 lowest RMSD structures from AbInitioVO (left) and AbInitio (right)
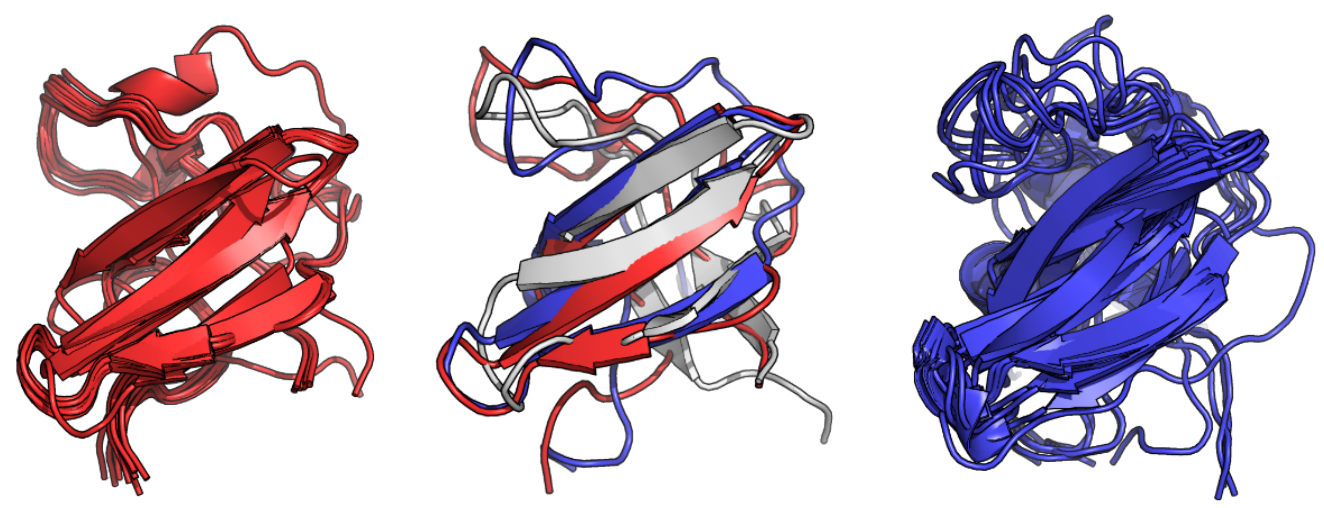

Figure S160: Dsh3 Lowest RMSD Full Structures. 10 lowest RMSD structures from AbInitioVO (left) and AbInitio (right) along with the overlay (center) of PDB 2A37 (white) and the single lowest RMSD structure from AbInitioVO (red) and AbInitio (blue)
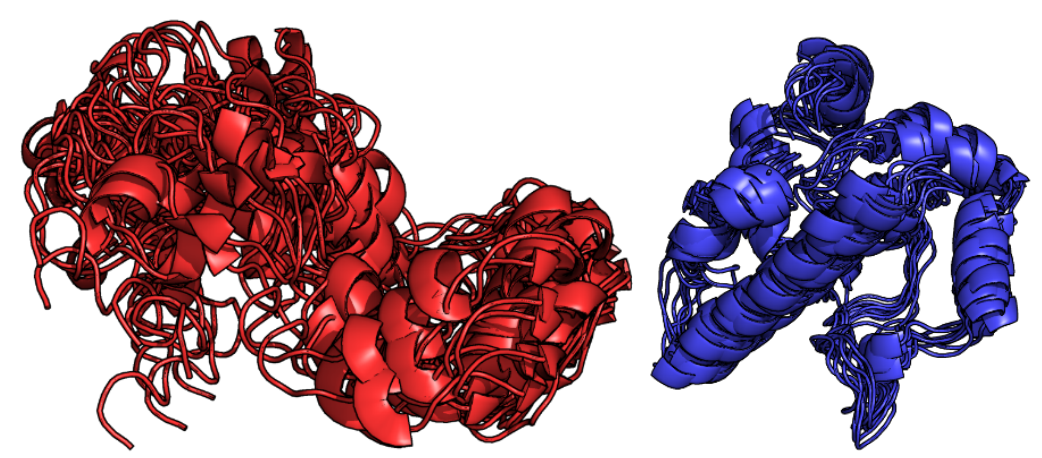

Figure S161: Ntal Lowest RMSD Full Structures. 10 lowest RMSD structures from AbInitioVO (left) and AbInitio (right) 

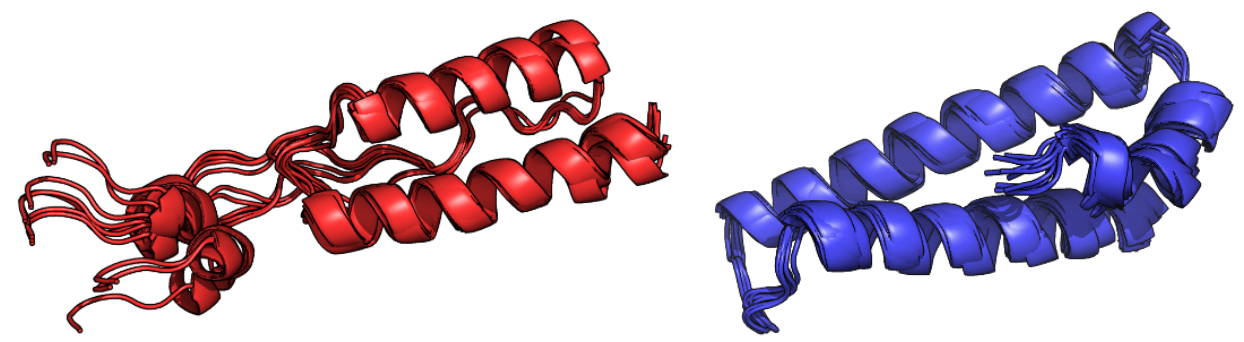

Figure S162: Paaa Lowest RMSD Full Structures. 10 lowest RMSD structures from AbInitioVO (left) and AbInitio (right)
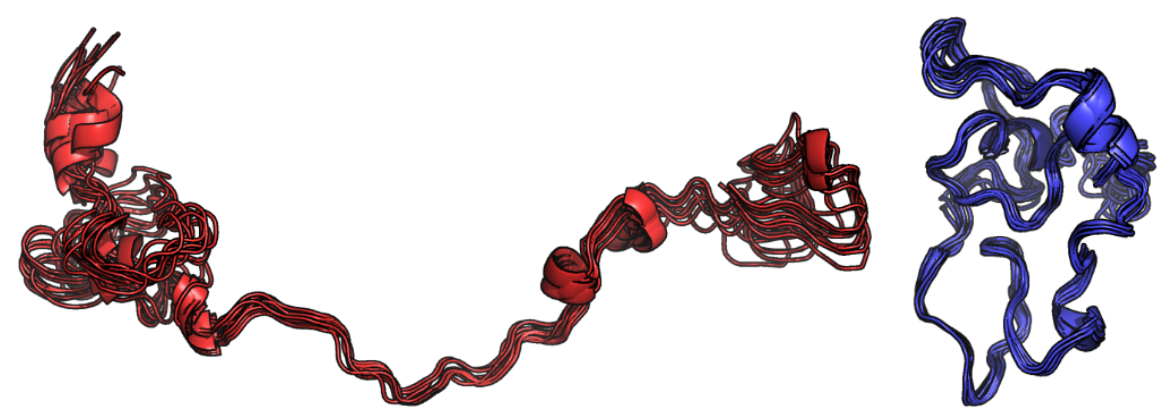

Figure S163: Sic1 Lowest RMSD Full Structures. 10 lowest RMSD structures from AbInitioVO (left) and AbInitio (right)
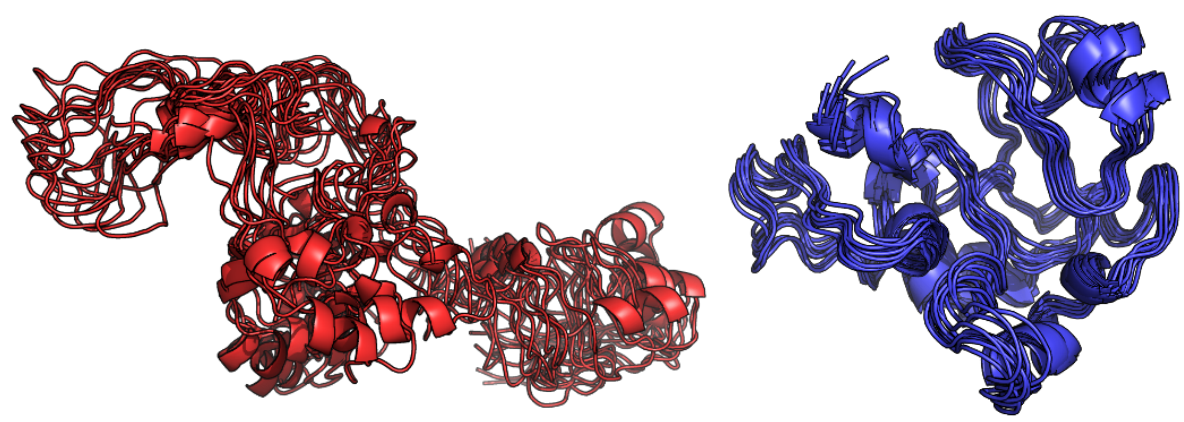

Figure S164: Tauk Lowest RMSD Full Structures. 10 lowest RMSD structures from AbInitioVO (left) and AbInitio (right) 
Comparison of FastFloppyTail and FastFloppyTail-Relax Simulations to Experimental Data

Summary of Simulated Proteins

Table S6: Comparison of FastFloppyTail and FastFloppyTail-Relax Outputs to Experimental Data

\begin{tabular}{|c|c|c|c|}
\hline $\begin{array}{l}\text { Protein } \\
\text { Experiment }\end{array}$ & FastFloppyTail & $\begin{array}{c}\text { FastFloppyTail } \\
\text { Relaxed }\end{array}$ & $\begin{array}{r}\text { Shaw } \\
\text { et. al. }\end{array}$ \\
\hline$\alpha$-synuclein ( $\alpha S$, asyn) & Best / Self & Best / Self & \\
\hline EFRET & $0.13 / 0.12$ & $0.10 / 0.11$ & - \\
\hline PRE & $0.20 / 0.20$ & $0.16 / 0.16$ & 0.17 \\
\hline DISTANCES & $11.5 / 10.8$ & $6.00 / 5.41$ & - \\
\hline \multicolumn{4}{|l|}{ J-COUPLINGS } \\
\hline${ }^{3} J_{\mathrm{HNH} \alpha}$ & $0.58 / 0.58$ & $0.67 / 0.67$ & 1.11 \\
\hline${ }^{1} J_{\mathrm{CaHa}}$ & $1.21 / 1.21$ & $1.14 / 1.13$ & - \\
\hline${ }^{1} J_{\mathrm{NC} a}$ & $0.35 / 0.35$ & $0.37 / 0.36$ & - \\
\hline${ }^{2} J_{\mathrm{NC} a}$ & $0.40 / 0.39$ & $0.39 / 0.39$ & - \\
\hline${ }^{3} J_{\mathbf{C}^{\prime}}{ }^{\prime}$ & $0.25 / 0.26$ & $0.27 / 0.27$ & 0.18 \\
\hline \multicolumn{4}{|l|}{ CHEMICAL SHIFTS } \\
\hline $\mathbf{C}$ & $0.43 / 0.44$ & $0.48 / 0.49$ & 0.31 \\
\hline CA & $0.34 / 0.34$ & $0.38 / 0.38$ & 0.51 \\
\hline CB & $1.02 / 1.02$ & $1.03 / 1.03$ & 1.04 \\
\hline $\mathbf{N}$ & $1.54 / 1.56$ & $1.62 / 1.63$ & 1.46 \\
\hline NH & $0.12 / 0.12$ & $0.12 / 0.11$ & 0.14 \\
\hline RDC & $0.41 / 0.40$ & $0.41 / 0.41$ & 0.41 \\
\hline $\mathbf{R}_{\mathrm{g}}(33.0 \pm 3.0,26.6 \pm 0.5)$ & $39.3 / 38.6$ & $32.3 / 31.3$ & 36.76 \\
\hline Ntail (ntal) & Best / Self & Best / Self & \\
\hline \multicolumn{4}{|l|}{ CHEMICAL SHIFTS } \\
\hline $\mathbf{C A}$ & $0.57 / 0.40$ & $0.58 / 0.40$ & 0.44 \\
\hline HN & $0.13 / 0.11$ & $0.13 / 0.11$ & 0.12 \\
\hline $\mathbf{N}$ & $0.90 / 0.83$ & $0.94 / 0.87$ & 0.89 \\
\hline $\mathbf{C}$ & $0.66 / 0.49$ & $0.69 / 0.52$ & 0.41 \\
\hline CB & $0.46 / 0.36$ & $0.48 / 0.38$ & 0.37 \\
\hline RDC & $0.48 / 0.39$ & $0.48 / 0.40$ & 0.95 \\
\hline $\mathbf{R}_{\mathrm{g}}(\mathbf{2 7 . 5} \pm \mathbf{0 . 7 0})$ & $37.5 / 36.9$ & $32.9 / 32.3$ & 26.65 \\
\hline Sic1 (sic1) & Best / Self & Best / Self & \\
\hline \multicolumn{4}{|l|}{ CHEMICAL SHIFTS } \\
\hline CA & $0.71 / 0.71$ & $0.68 / 0.67$ & 0.77 \\
\hline $\mathbf{H N}$ & $0.15 / 0.15$ & $0.15 / 0.15$ & 0.12 \\
\hline HA & $0.24 / 0.24$ & $0.25 / 0.25$ & 0.25 \\
\hline $\mathbf{N}$ & $1.60 / 1.57$ & $1.69 / 1.65$ & 1.16 \\
\hline CB & $0.51 / 0.52$ & $0.55 / 0.56$ & 0.52 \\
\hline RDC & $0.44 / 0.44$ & $0.44 / 0.44$ & 0.43 \\
\hline$R_{g}(31.2 \pm 0.8)$ & $28.4 / 28.5$ & $24.9 / 25.3$ & 24.42 \\
\hline
\end{tabular}




\begin{tabular}{|c|c|c|c|}
\hline Tau K18 (tauk) & Best / Self & Best / Self & \\
\hline \multicolumn{4}{|c|}{ CHEMICAL SHIFTS } \\
\hline $\mathbf{C}$ & $0.41 / 0.47$ & $0.45 / 0.51$ & - \\
\hline CB & $0.34 / 0.37$ & $0.34 / 0.37$ & - \\
\hline $\mathbf{C A}$ & $0.33 / 0.42$ & $0.38 / 0.47$ & - \\
\hline $\mathbf{N}$ & $1.67 / 1.69$ & $1.68 / 1.69$ & - \\
\hline HN & $0.13 / 0.14$ & $0.13 / 0.15$ & - \\
\hline RDC & $0.39 / 0.41$ & $0.40 / 0.41$ & - \\
\hline $\mathbf{R}_{\mathrm{g}}(33.4 \pm 7.2)$ & $40.1 / 39.7$ & $34.7 / 34.5$ & - \\
\hline DrkN Sh3 (dsh3) & Best / Self & Best / Self & \\
\hline \multicolumn{4}{|c|}{ CHEMICAL SHIFTS } \\
\hline $\mathbf{C}$ & $0.98 / 0.77$ & $0.93 / 0.74$ & 0.65 \\
\hline CB & $0.48 / 0.51$ & $0.47 / 0.51$ & 0.50 \\
\hline $\mathbf{C A}$ & $0.74 / 0.68$ & $0.70 / 0.71$ & 0.50 \\
\hline HN & $0.29 / 0.22$ & $0.28 / 0.22$ & 0.23 \\
\hline HA & $0.12 / 0.13$ & $0.11 / 0.14$ & 0.10 \\
\hline RDC & $0.38 / 0.39$ & $0.37 / 0.39$ & 0.91 \\
\hline${ }^{3} \boldsymbol{J}_{\mathrm{HNH} \alpha}$ & $0.89 / 1.01$ & $0.86 / 1.13$ & 1.08 \\
\hline PRE & $0.33 / 0.33$ & $0.30 / 0.30$ & 0.3 \\
\hline$R_{g}(18.3)$ & $20.6 / 23.6$ & $19.2 / 22.2$ & 19.46 \\
\hline PaaA2 (paaa) & Best / Self & Best / Self & \\
\hline \multicolumn{4}{|c|}{ CHEMICAL SHIFTS } \\
\hline $\mathbf{C}$ & $0.57 / 0.71$ & $0.55 / 0.68$ & 0.66 \\
\hline CB & $0.46 / 0.55$ & $0.44 / 0.53$ & 0.37 \\
\hline $\mathbf{C A}$ & $0.79 / 0.99$ & $0.79 / 0.97$ & 0.64 \\
\hline $\mathbf{N}$ & $1.38 / 1.44$ & $1.39 / 1.55$ & 0.99 \\
\hline HN & $0.18 / 0.21$ & $0.18 / 0.20$ & 0.17 \\
\hline HA & $0.10 / 0.12$ & $0.10 / 0.12$ & 0.10 \\
\hline RDC & $0.35 / 0.36$ & $0.35 / 0.36$ & 0.76 \\
\hline $\mathbf{R}_{\mathrm{g}}(22.4 \pm 4.0)$ & $23.6 / 23.5$ & $22.4 / 22.4$ & 21.39 \\
\hline
\end{tabular}

*All values reported above are computed at RMSD values except the reported Rg values which represent the average $\operatorname{Rg}(\AA)$ and RDC values which are computed as Q-values, as described in Zweckstetter et. al. ${ }^{12}$. 


\section{Comparisons of $\alpha S$ Data}
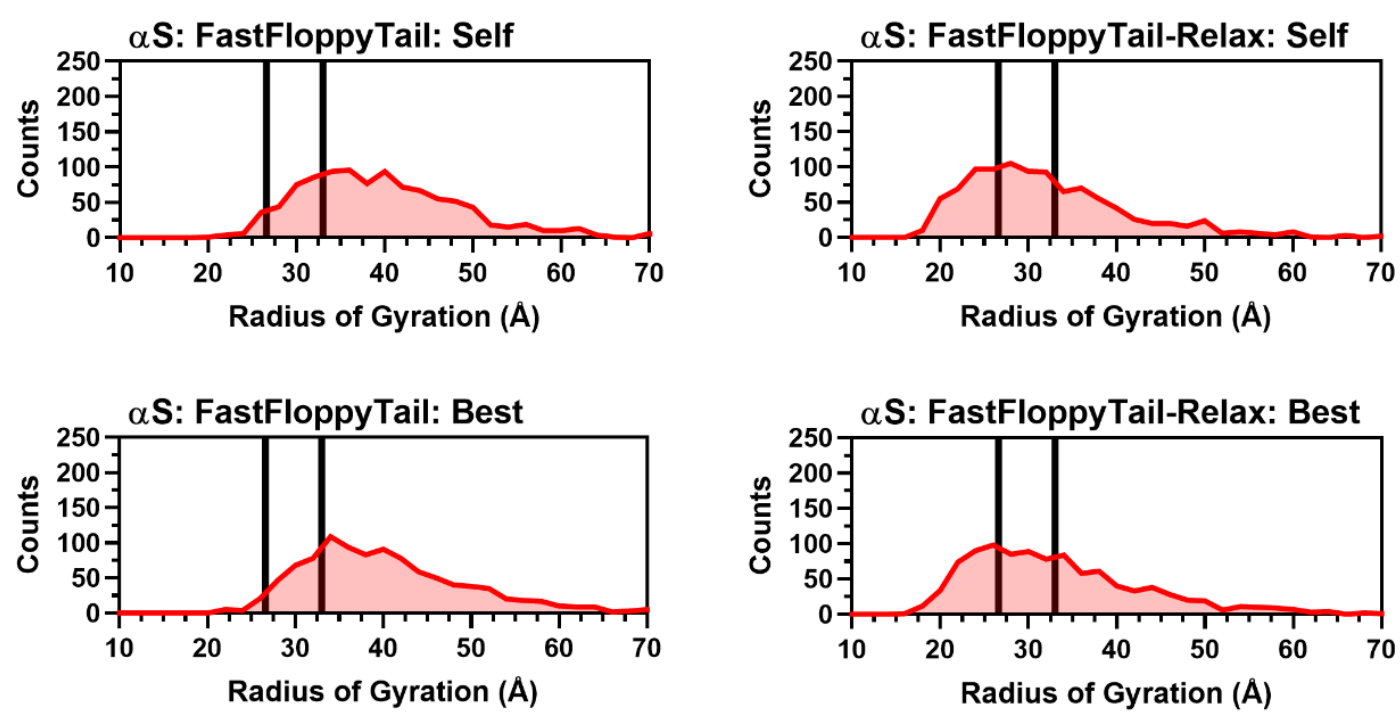

Figure S165: Histograms of Radii of Gyration for $\alpha$ S. Histograms of the radius of gyration from self (Top Left) and best (Top Right) reweighting in FastFloppyTail and self (Bottom Left) and best (Bottom Right) reweighting in FastFloppyTail-Relax compared to experimental values from SAXS ${ }^{19}$ and NMR ${ }^{20}$ (black).
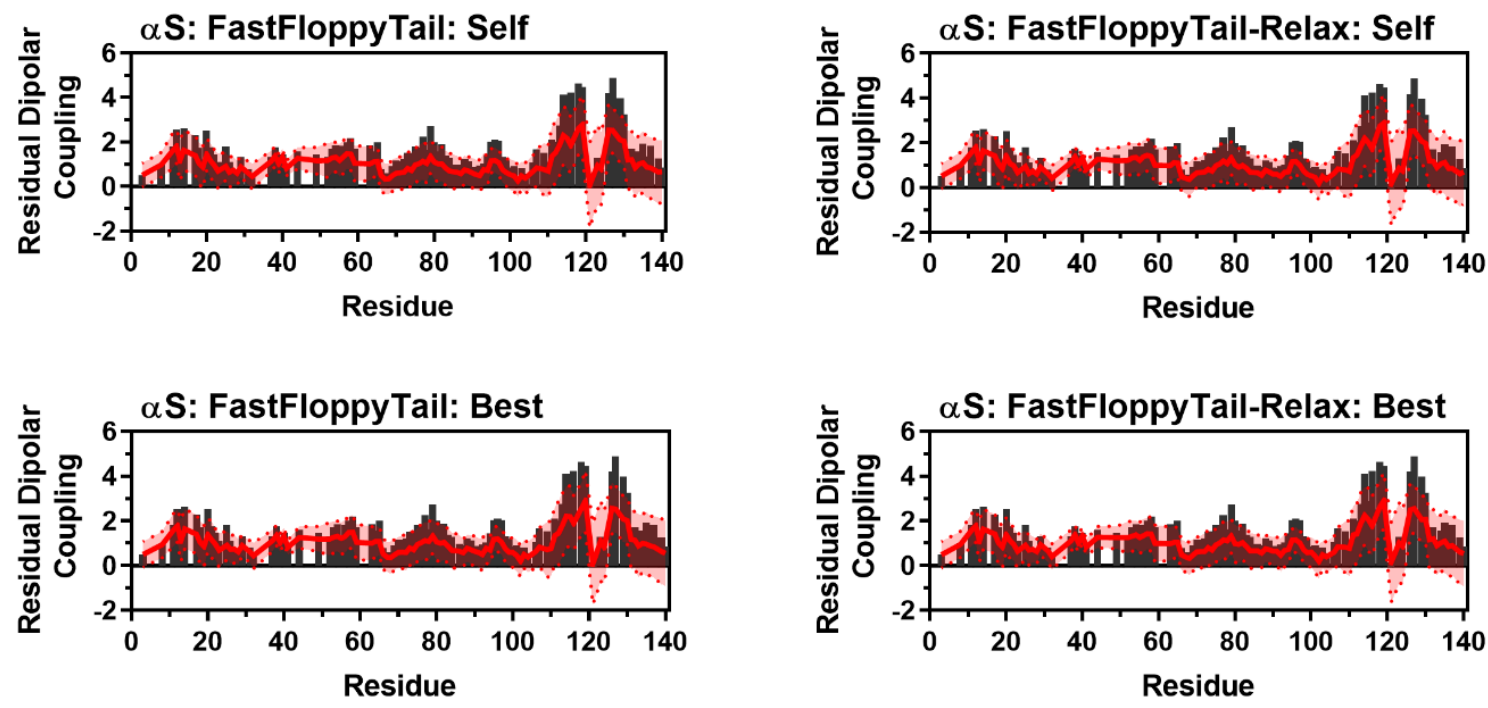

Figure S166: Comparison of Simulated and Experimental Residual Dipolar Coupling Data. Simulated RDC values (red line) from self (Top Left) and best (Top Right) reweighting in FastFloppyTail and self (Bottom Left) and best (Bottom Right) reweighting in FastFloppyTailRelax overlayed on experimental data (black bars) from Bertoncini et al. ${ }^{25}$. 

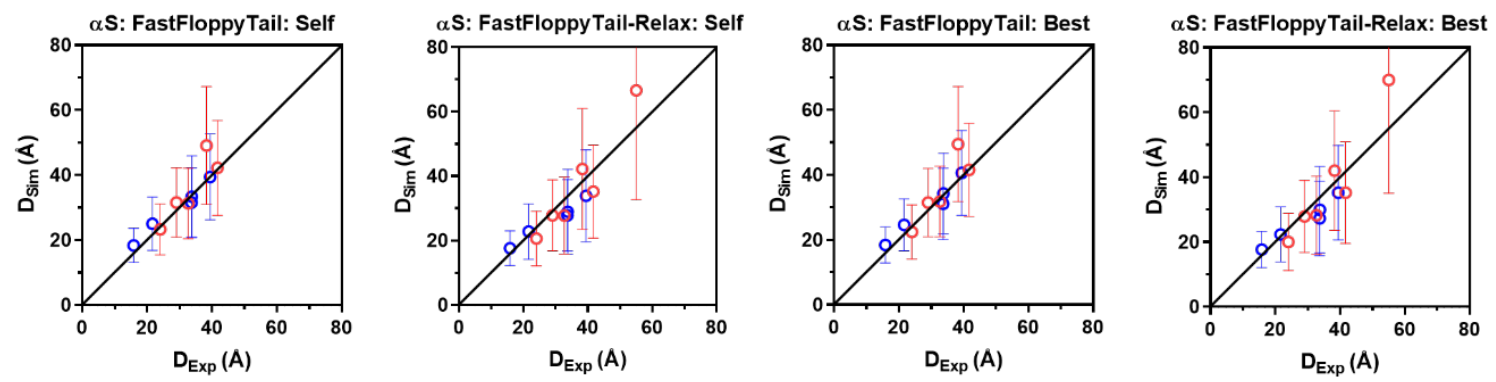

Figure S167: Comparison of Simulated Distance with Experimental Distances. Simulated distances for self-reweighting in FastFloppyTail (Left) and FastFloppyTail-Relax (Middle Left) and best-reweighting in FastFloppyTail (Middle Right) and FastFloppyTail-Relax (Far Right) with data from Lee et al. ${ }^{24}$ (Red) and Grupi et al. ${ }^{23}$ (Blue).
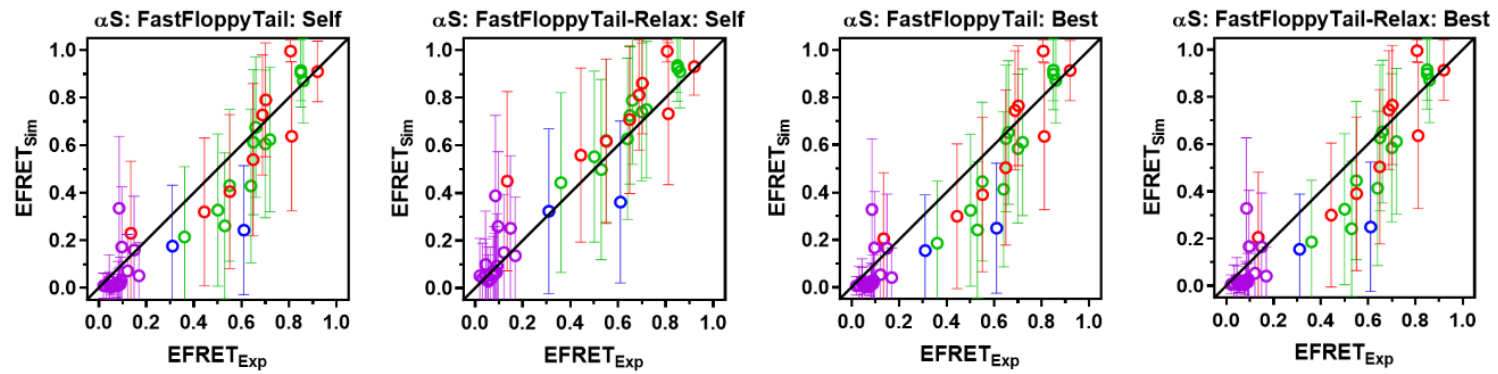

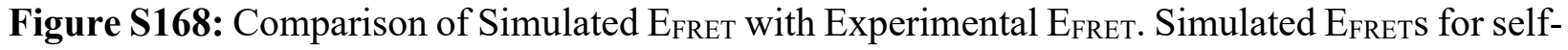
reweighting in FastFloppyTail (Left) and FastFloppyTail-Relax (Middle Left) and bestreweighting in FastFloppyTail (Middle Right) and FastFloppyTail-Relax (Far Right) with data from Ferrie et al. Cnf-Trp ${ }^{21}$ (Purple) and Fam-Raz ${ }^{21}$ (Red) Pairs, Ferrie et al. Mcm-Acd pair ${ }^{22}$ (Blue), and Nath et al. ${ }^{1}$ (Green). 


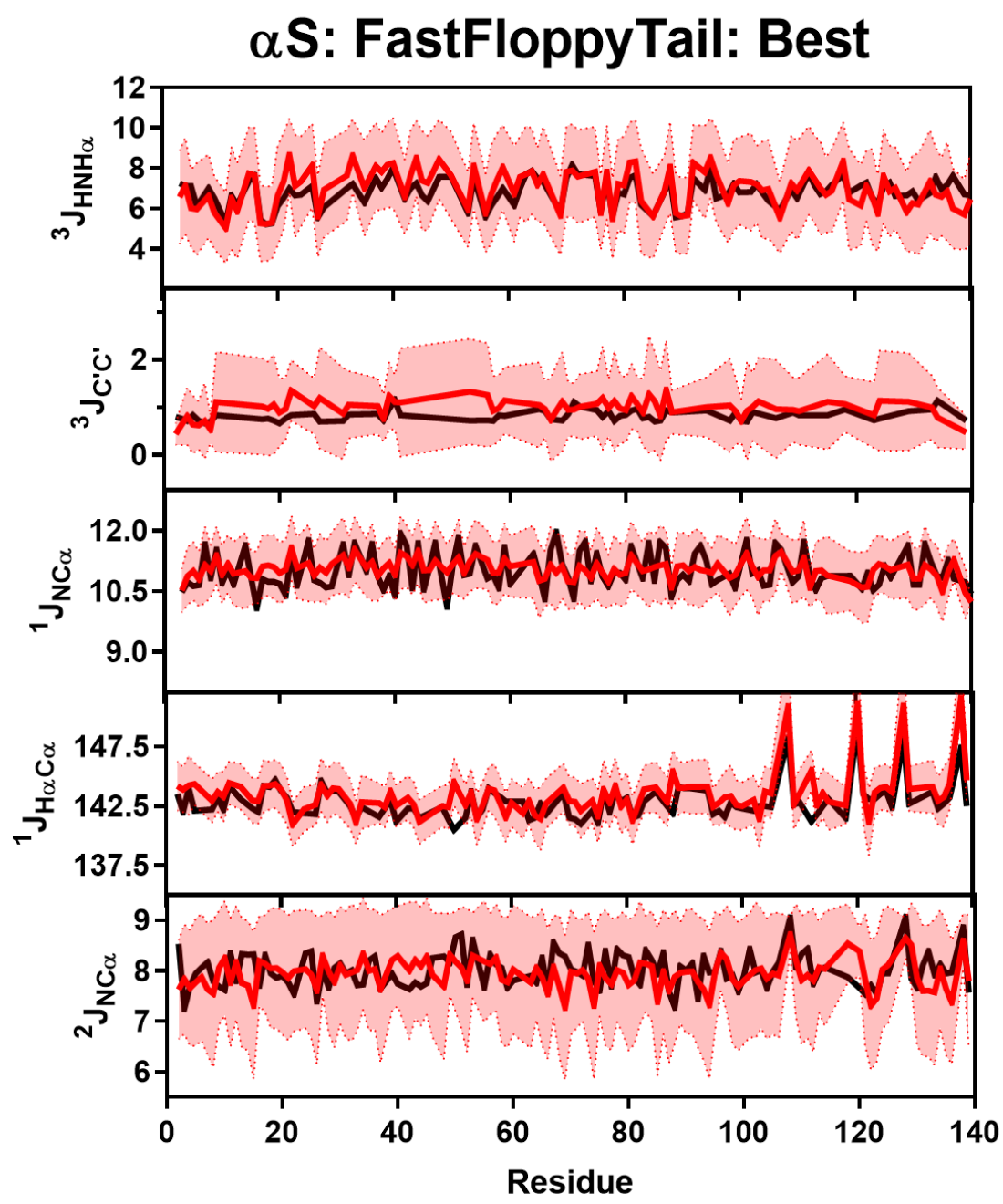

Figure S169: Comparison of Simulated and Experimental J-Coupling Data. Simulated J-Coupling values from the FastFloppyTail simulations with best-reweighting (red) overlayed on experimental data (black) of ${ }^{3} \mathbf{J}_{\mathrm{HNH} \alpha}$ (Row 1), ${ }^{3} \mathbf{J}_{\mathrm{CC}}$ (Row 2), ${ }^{1} \mathbf{J}_{\mathrm{H} \alpha \mathrm{C} \alpha}$ (Row 3), ${ }^{1} \mathbf{J}_{\mathrm{NC} \alpha}$ (Row 4) and ${ }^{2} \mathbf{J}_{\mathrm{NC} \alpha}$ (Row 5) from Mantsyzov et al. and Lee et. al. ${ }^{13,18}$. 


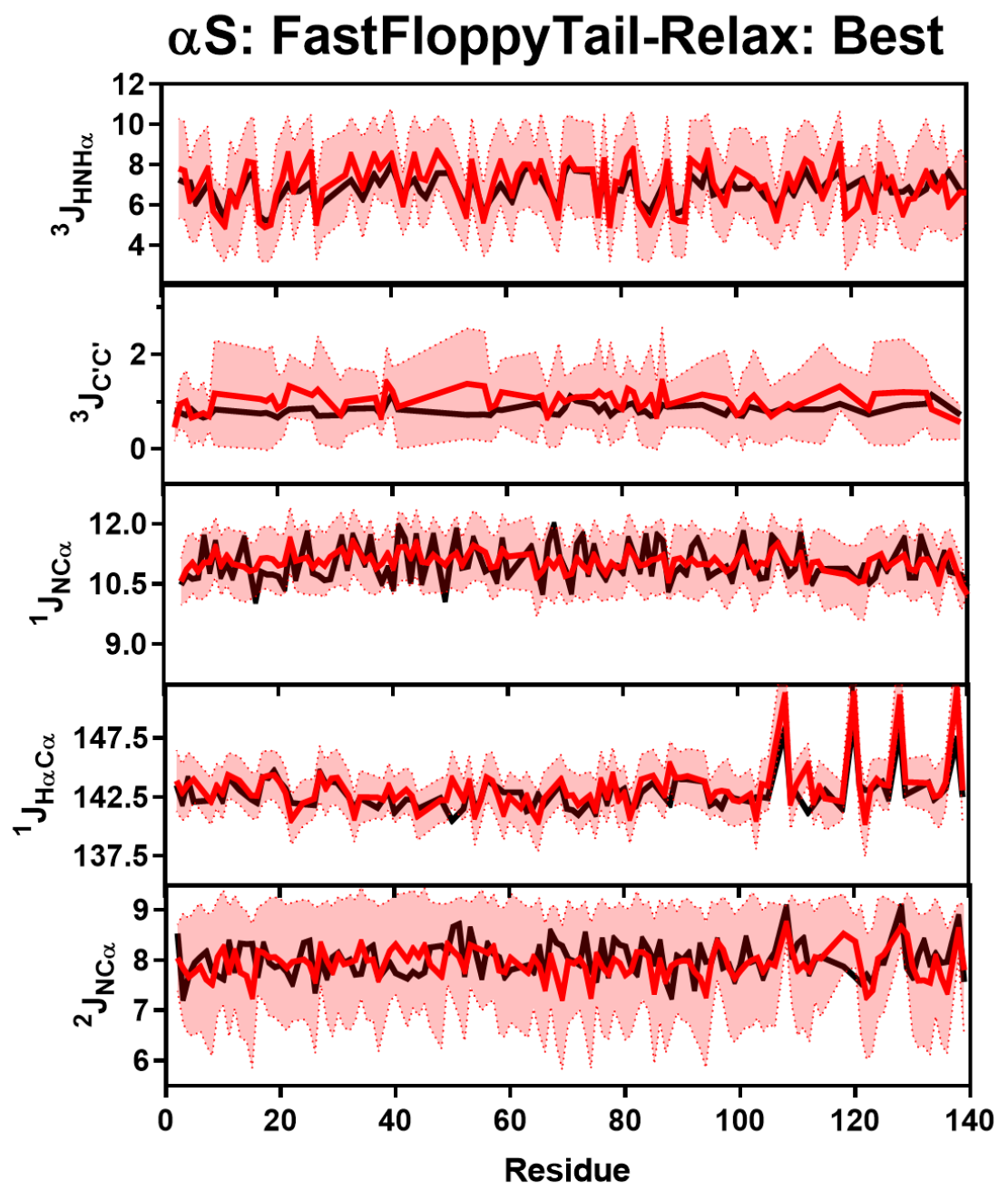

Figure S170: Comparison of Simulated and Experimental J-Coupling Data. Simulated J-Coupling values from the FastFloppyTail-Relax simulations with best-reweighting (red) overlayed on experimental data (black) of ${ }^{3} \mathbf{J}_{\mathrm{HNH} \alpha}$ (Row 1), ${ }^{3} \mathbf{J}_{\mathrm{CC}}$ (Row 2), ${ }^{1} \mathbf{J}_{\mathrm{H} \alpha \mathrm{C} \alpha}$ (Row 3), ${ }^{1} \mathbf{J}_{\mathrm{NC} \alpha}$ (Row 4) and ${ }^{2} \mathbf{J}_{\mathrm{NC} \alpha}$ (Row 5) from Mantsyzov et al. and Lee et. al. ${ }^{13,18 .}$ 


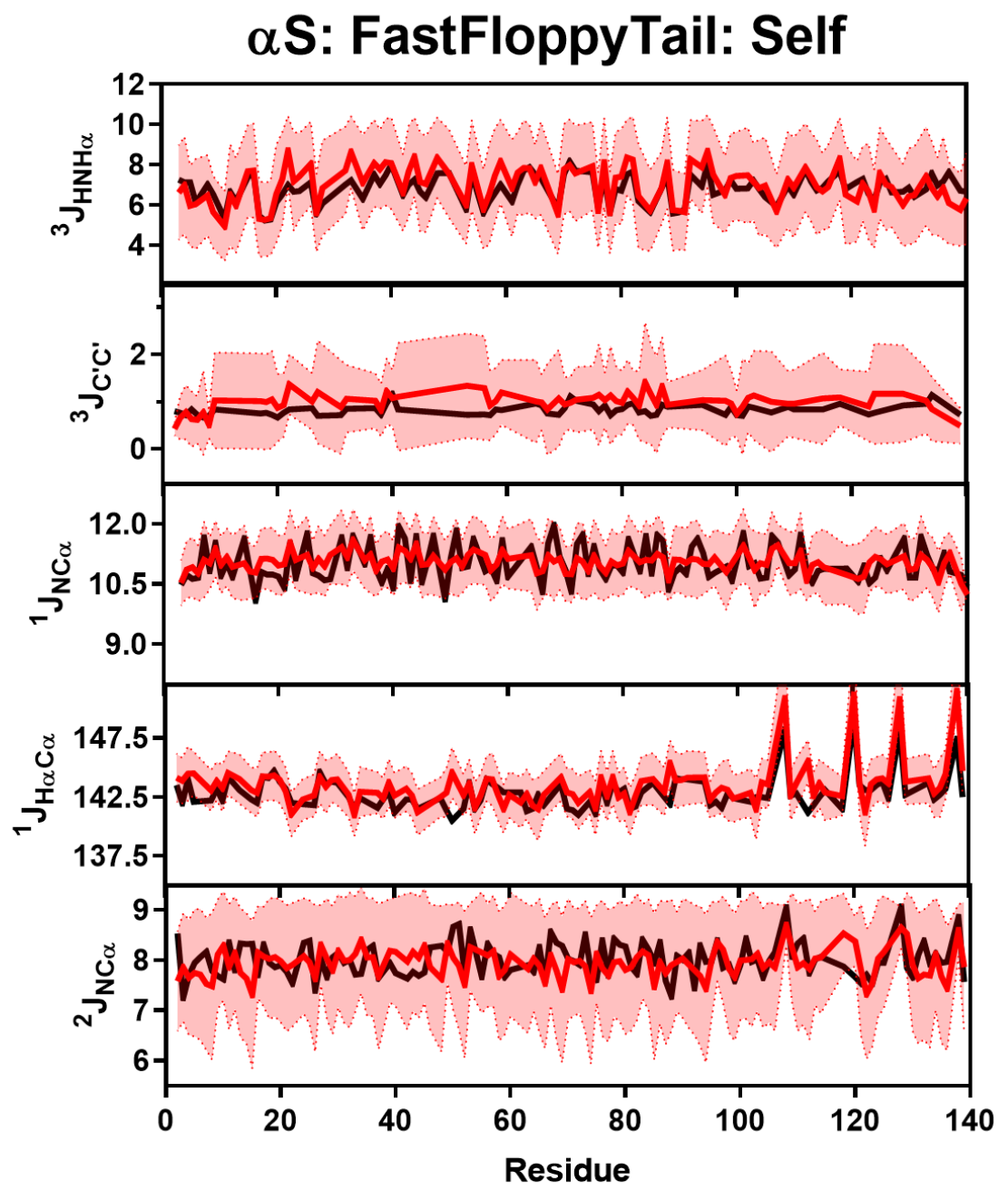

Figure S171: Comparison of Simulated and Experimental J-Coupling Data. Simulated J-Coupling values from the FastFloppyTail simulations with self-reweighting (red) overlayed on experimental data (black) of ${ }^{3} \mathrm{~J}_{\mathrm{HNH} \alpha}$ (Row 1), ${ }^{3} \mathrm{~J}_{\mathrm{CC}}$ (Row 2), ${ }^{1} \mathbf{J}_{\mathrm{H} \alpha \mathrm{C} \alpha}$ (Row 3), ${ }^{1} \mathbf{J}_{\mathrm{NC} \alpha}$ (Row 4) and ${ }^{2} \mathbf{J}_{\mathrm{NC} \alpha}$ (Row 5) from Mantsyzov et al. and Lee et. al. ${ }^{13,18}$. 


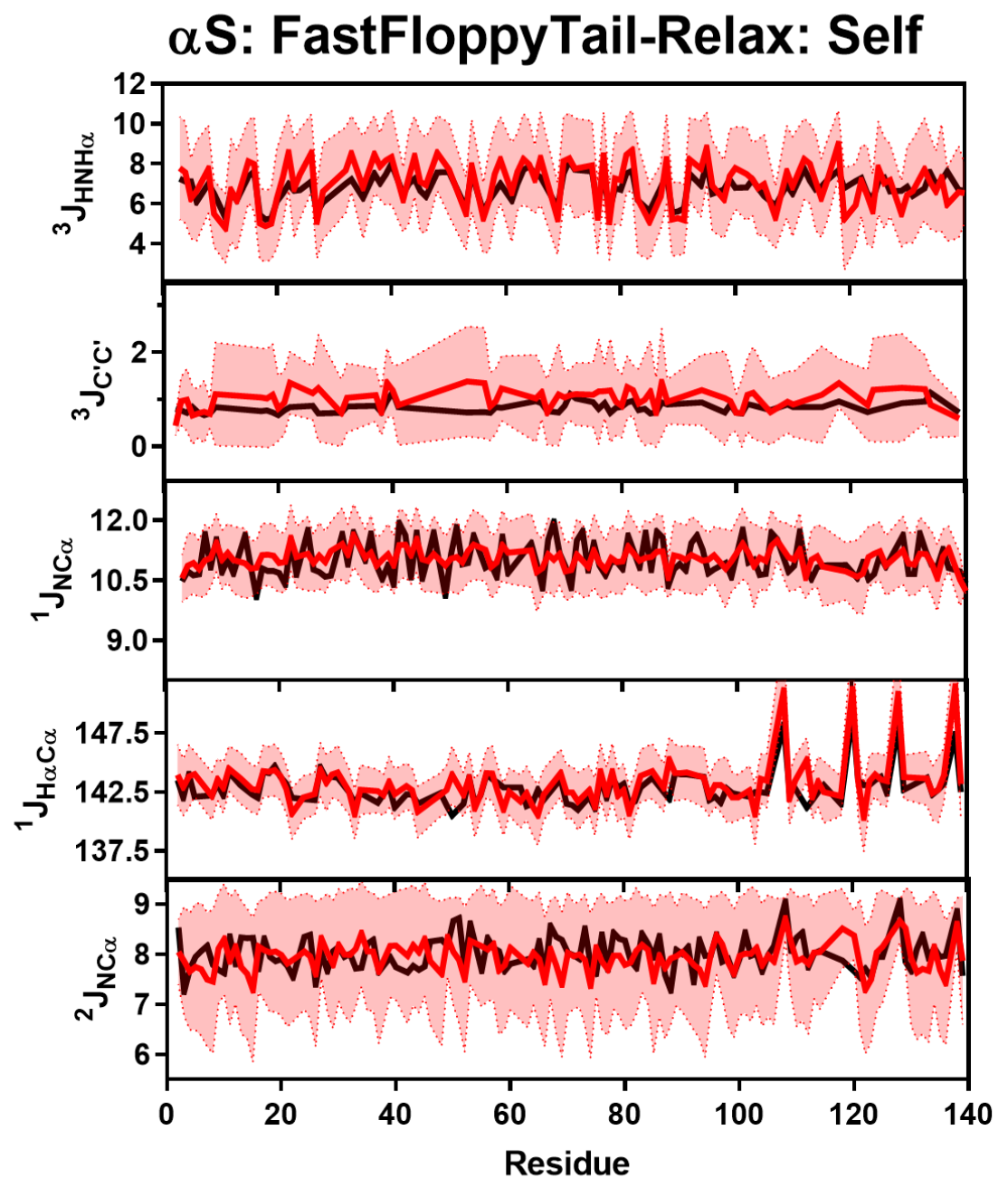

Figure S172: Comparison of Simulated and Experimental J-Coupling Data. Simulated J-Coupling values from the FastFloppyTail-Relax simulations with self-reweighting (red) overlayed on experimental data (black) of ${ }^{3} J_{\mathrm{HNH} \alpha}$ (Row 1), ${ }^{3} \mathrm{~J}_{\mathrm{CC}}$ (Row 2), ${ }^{1} \mathrm{~J}_{\mathrm{H} \alpha \mathrm{C} \alpha}$ (Row 3), ${ }^{1} \mathrm{~J}_{\mathrm{NC} \alpha}$ (Row 4) and ${ }^{2} \mathrm{~J}_{\mathrm{NC} \alpha}$ (Row 5) from Mantsyzov et al. and Lee et. al. ${ }^{13,18}$. 


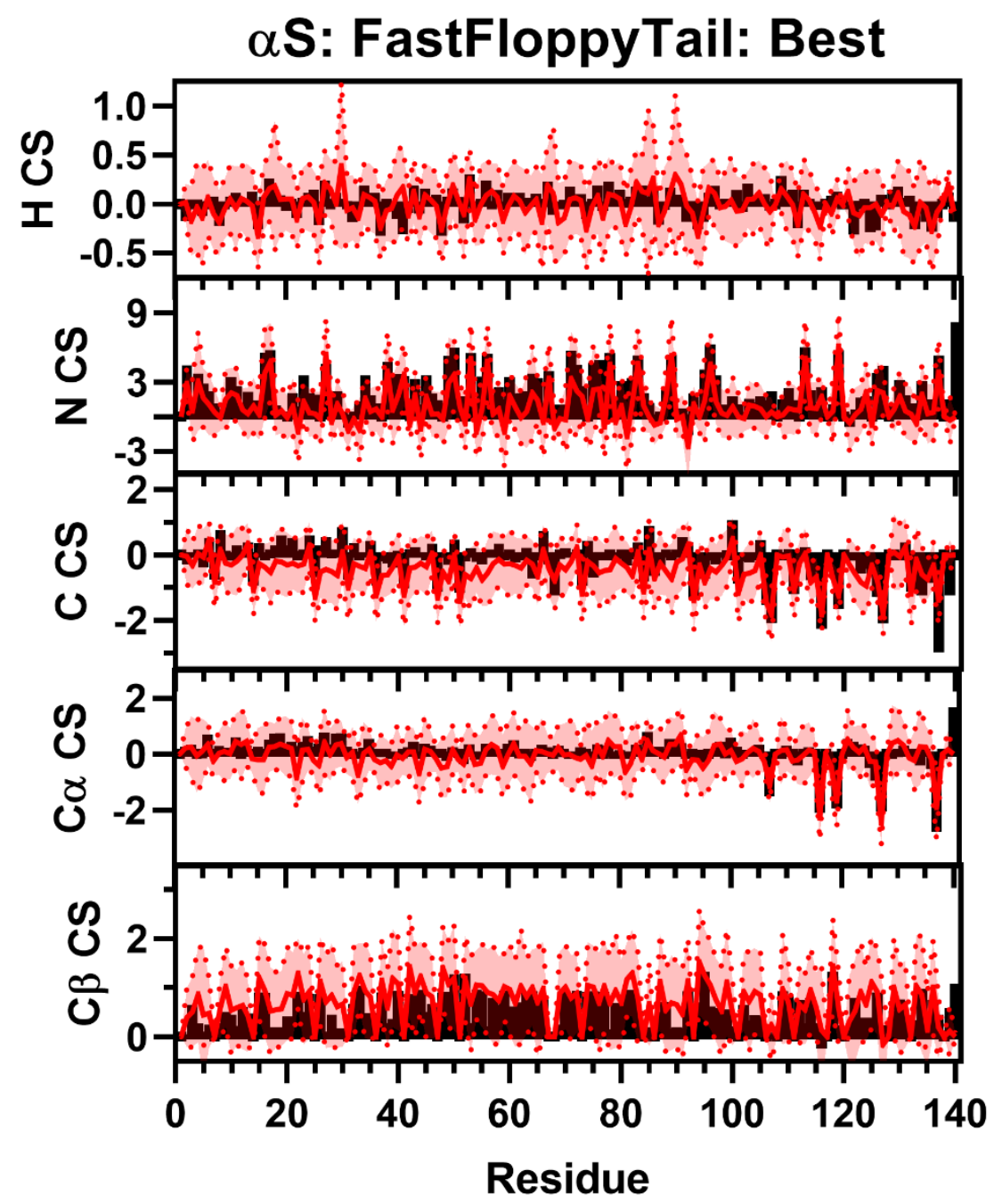

Figure S173: Comparison of Simulated and Experimental NMR Secondary Chemical Shift Data for $\alpha \mathrm{S}$. Simulated Chemical Shift values from the FastFloppyTail ensemble using best-reweighted fragment selection (red) overlayed on experimental data (black bars) of N (Row 1), H (Row 2), C (Row 3), $\mathrm{C} \alpha$ (Row 4), and C $\beta$ (Row 5) chemical shifts from Sung et al. ${ }^{8}$. Neighbor corrected random coil chemical shift values from SPARTA+ have been subtracted from both simulated and experimental chemical shifts to generate secondary chemical shift data ${ }^{11}$. 


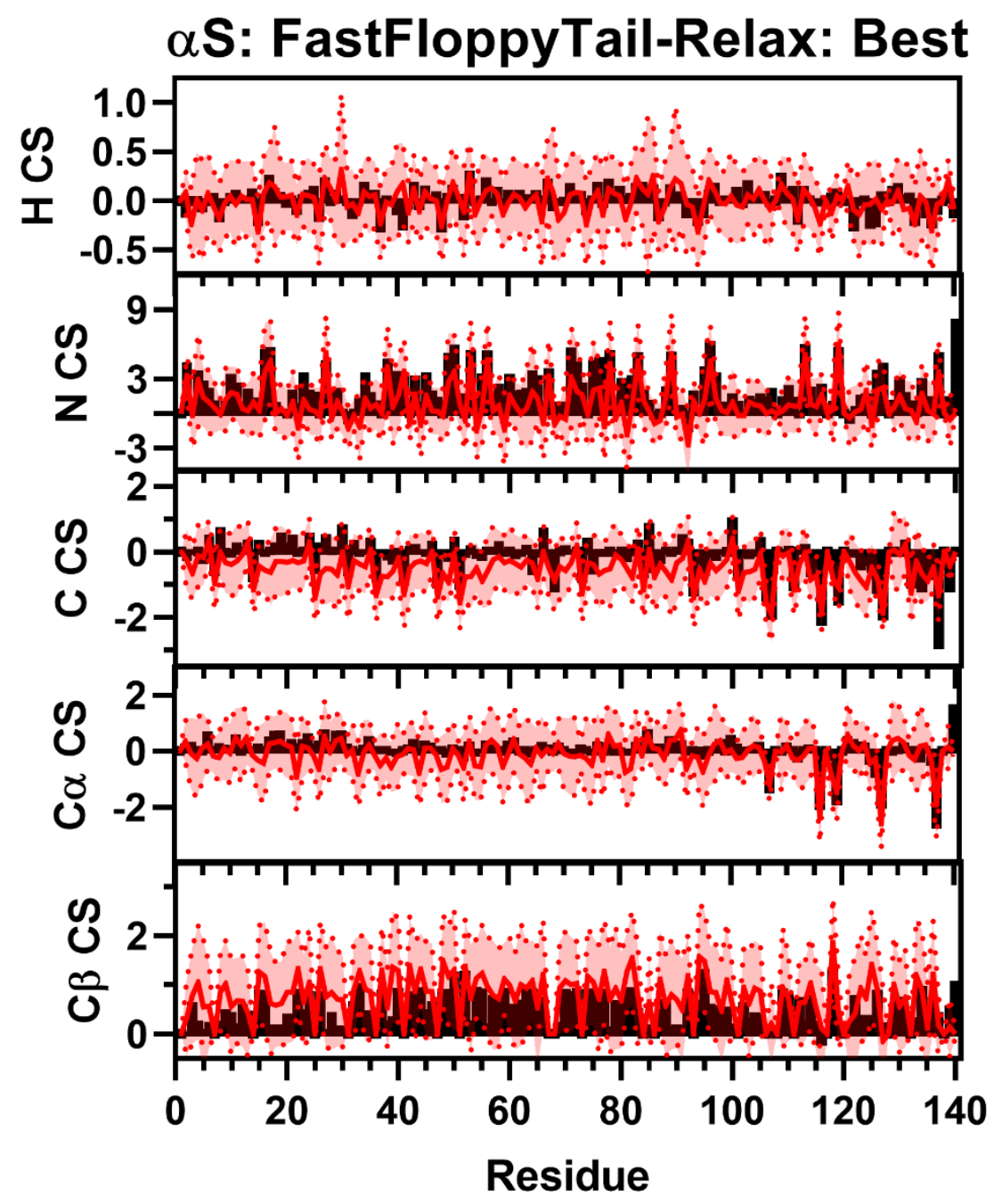

Figure S174: Comparison of Simulated and Experimental NMR Secondary Chemical Shift Data for $\alpha \mathrm{S}$. Simulated Chemical Shift values from the FastFloppyTail-Relax ensemble using bestreweighted fragment selection (red) overlayed on experimental data (black bars) of $\mathrm{N}$ (Row 1), $\mathrm{H}$ (Row 2), C (Row 3), C $\alpha$ (Row 4), and C $\beta$ (Row 5) chemical shifts from Sung et al. ${ }^{8}$ Neighbor corrected random coil chemical shift values from SPARTA+ have been subtracted from both simulated and experimental chemical shifts to generate secondary chemical shift data ${ }^{11}$. 


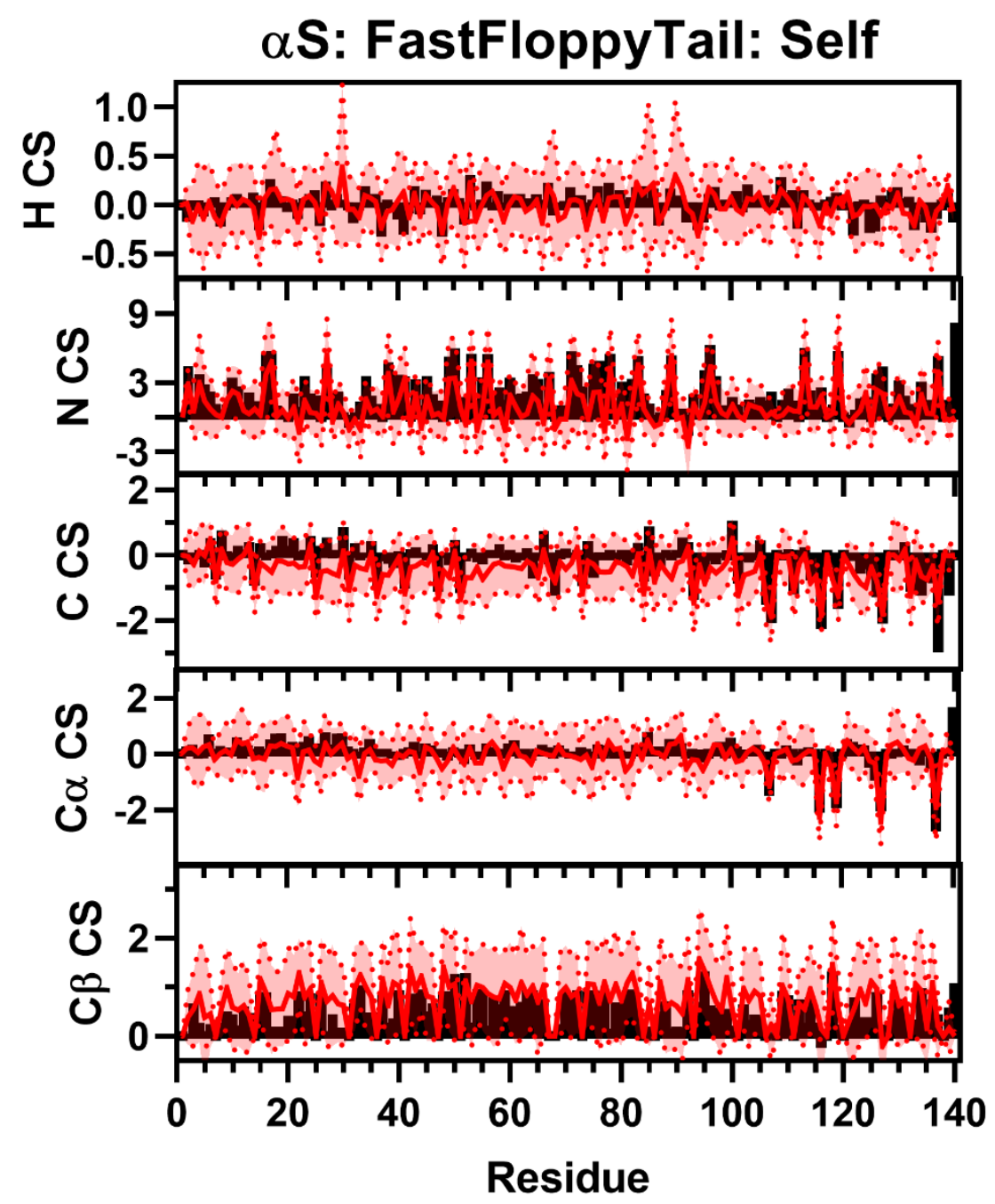

Figure S175: Comparison of Simulated and Experimental NMR Secondary Chemical Shift Data for $\alpha \mathrm{S}$. Simulated Chemical Shift values from the FastFloppyTail ensemble using self-reweighted fragment selection (red) overlayed on experimental data (black bars) of N (Row 1), H (Row 2), C (Row 3), $\mathrm{C} \alpha$ (Row 4), and $\mathrm{C} \beta$ (Row 5) chemical shifts from Sung et al. ${ }^{8}$ Neighbor corrected random coil chemical shift values from SPARTA+ have been subtracted from both simulated and experimental chemical shifts to generate secondary chemical shift data ${ }^{11}$. 


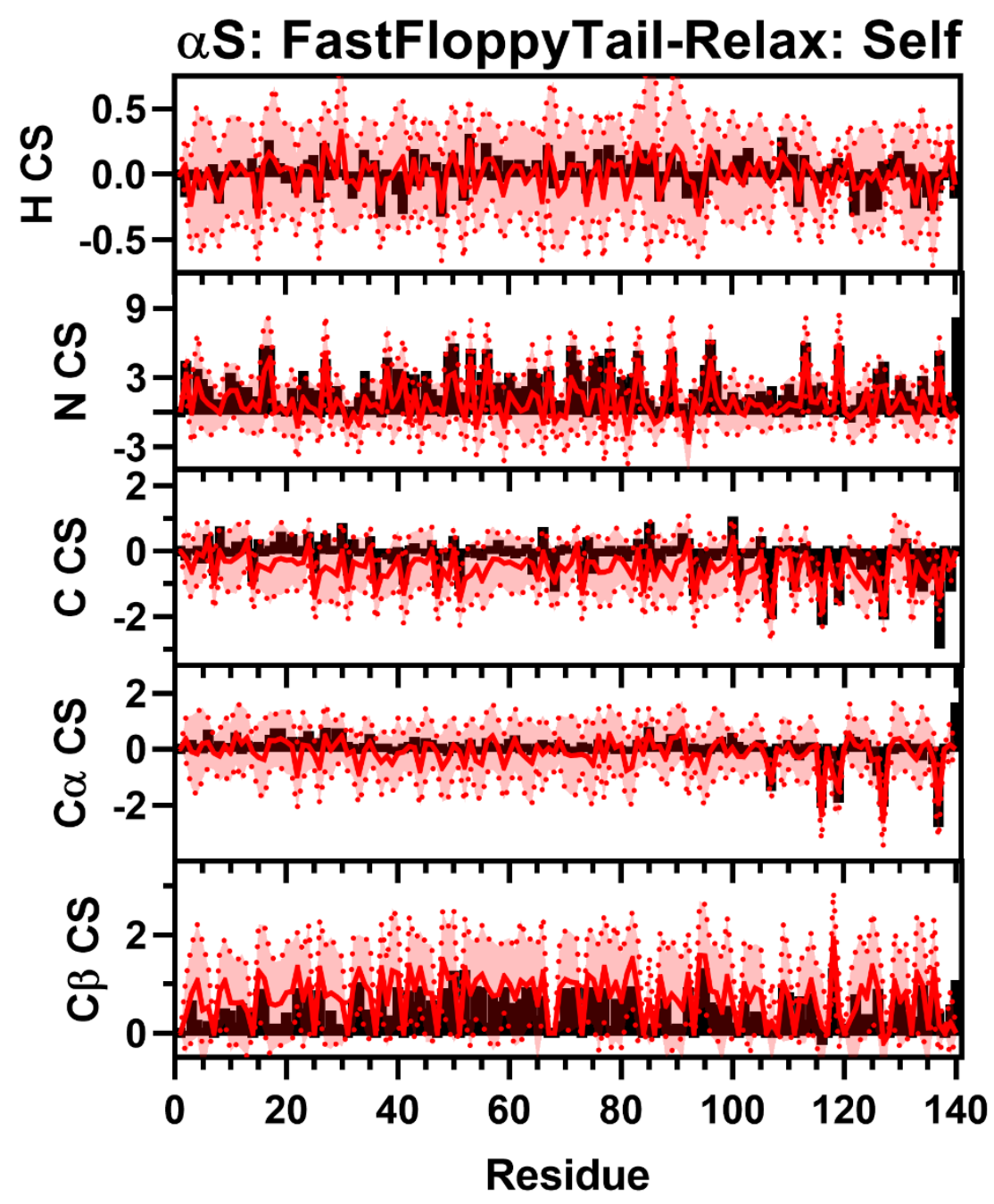

Figure S176: Comparison of Simulated and Experimental NMR Secondary Chemical Shift Data for $\alpha \mathrm{S}$. Simulated Chemical Shift values from the FastFloppyTail-Relax ensemble using selfreweighted fragment selection (red) overlayed on experimental data (black bars) of $\mathrm{N}$ (Row 1), $\mathrm{H}$ (Row 2), C (Row 3), C $\alpha$ (Row 4), and C $\beta$ (Row 5) chemical shifts from Sung et al. ${ }^{8}$ Neighbor corrected random coil chemical shift values from Sparta + have been subtracted from both simulated and experimental chemical shifts to generate secondary chemical shift data ${ }^{11}$. 


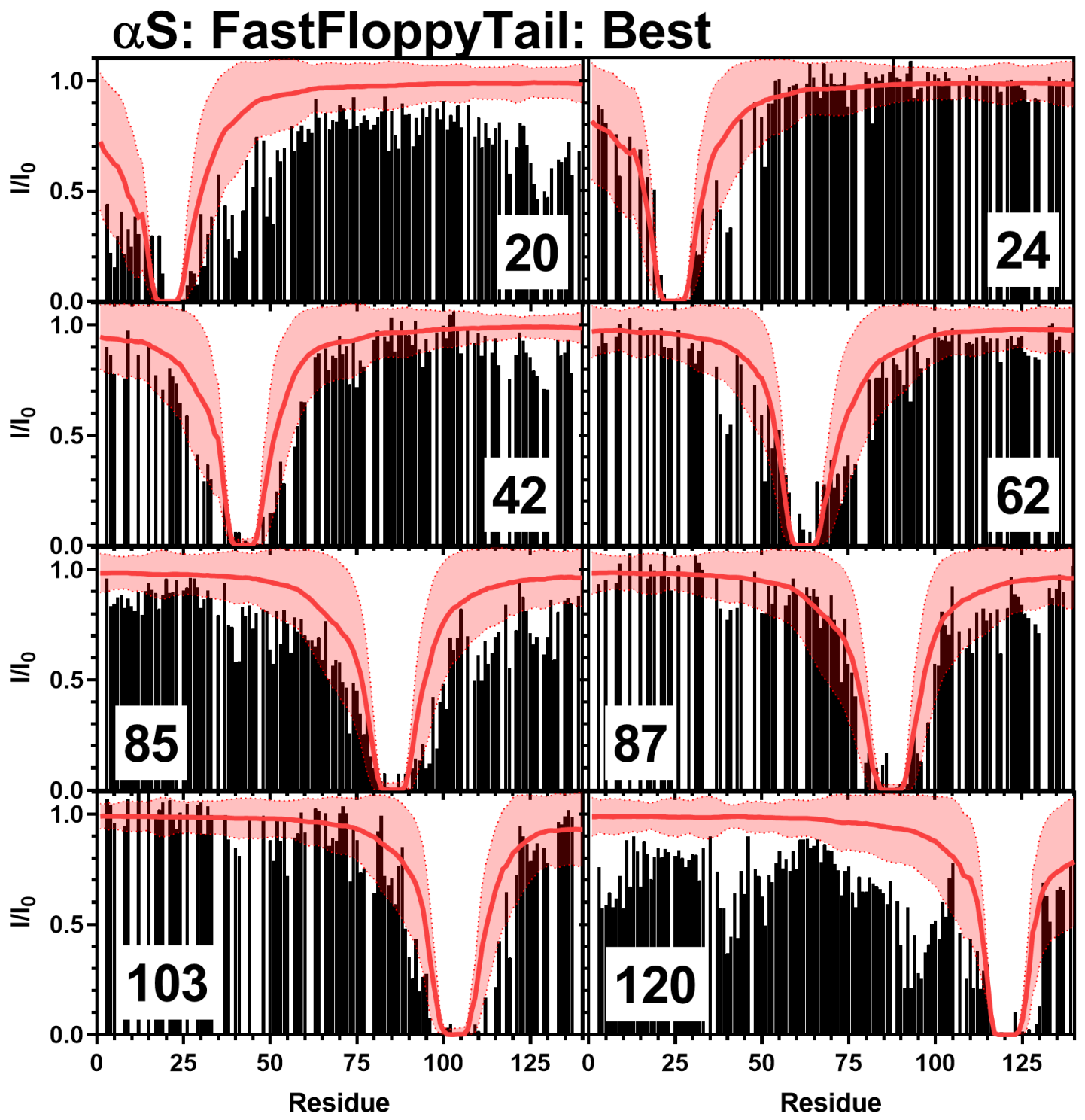

Figure S177: Comparison of Simulated PRE Values and Experimental PRE Values: Simulated PRE values from FastFloppyTail using best-reweighting (red line) overlayed on top of experimental data (black bars) from positions 20 (Top Left), 24 (Top Right), 42 (Upper Middle Left), 62 (Upper Middle Right), 85 (Lower Middle Left), 87 (Lower Middle Right), 103 (Bottom Left), 120 (Bottom Right). Experimental data for positions 20, 85, and 120 are from Sung et al. ${ }^{8}$ and data for positions $24,42,62,87$, and 103 are from Dedmon et al. ${ }^{9}$. 


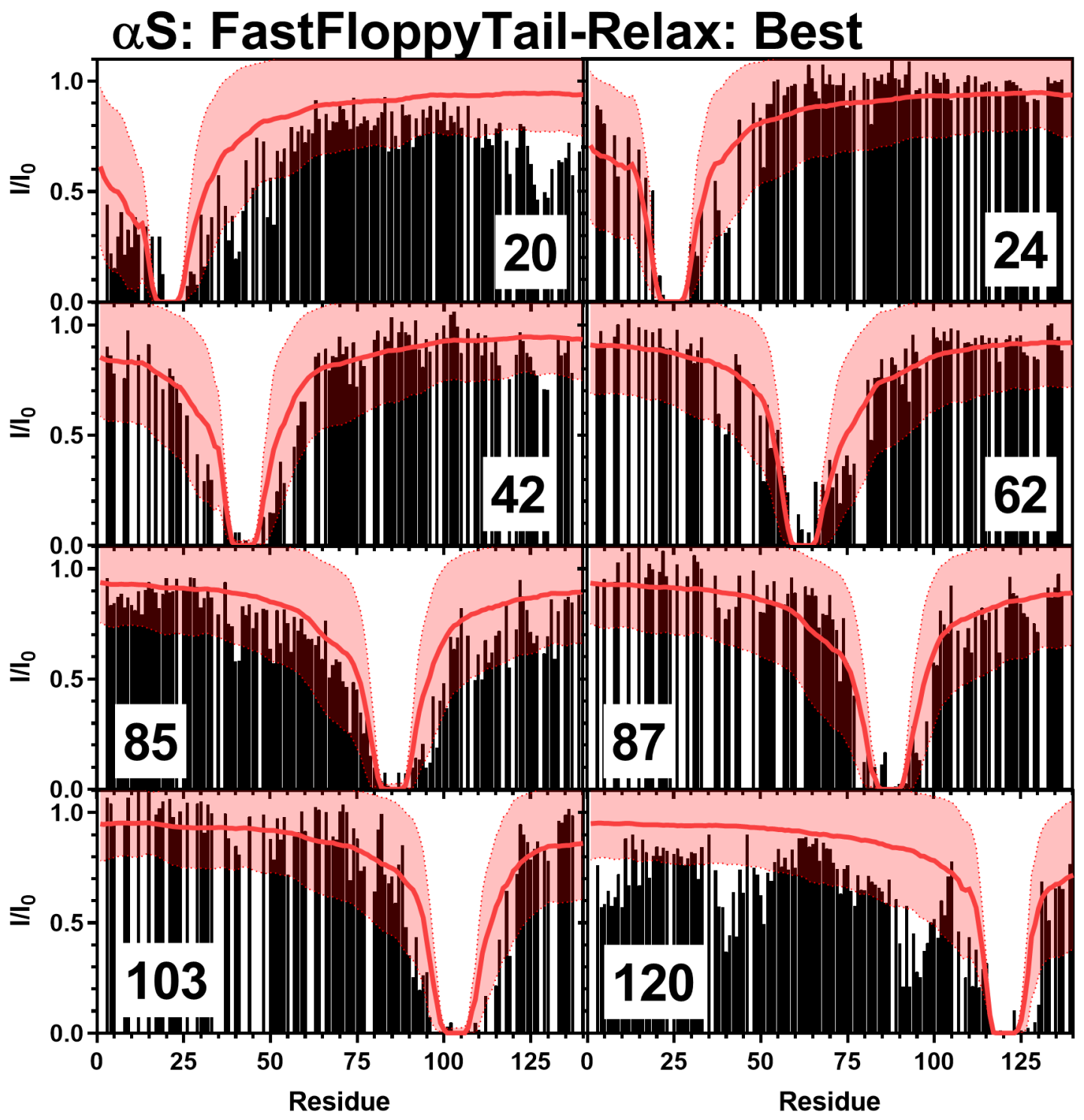

Figure S178: Comparison of Simulated PRE Values and Experimental PRE Values: Simulated PRE values from FastFloppyTail-Relax using best-reweighting (red line) overlayed on top of experimental data (black bars) from positions 20 (Top Left), 24 (Top Right), 42 (Upper Middle Left), 62 (Upper Middle Right), 85 (Lower Middle Left), 87 (Lower Middle Right), 103 (Bottom Left), 120 (Bottom Right). Experimental data for positions 20, 85, and 120 are from Sung et al. ${ }^{8}$ and data for positions $24,42,62,87$, and 103 are from Dedmon et al. ${ }^{9}$. 


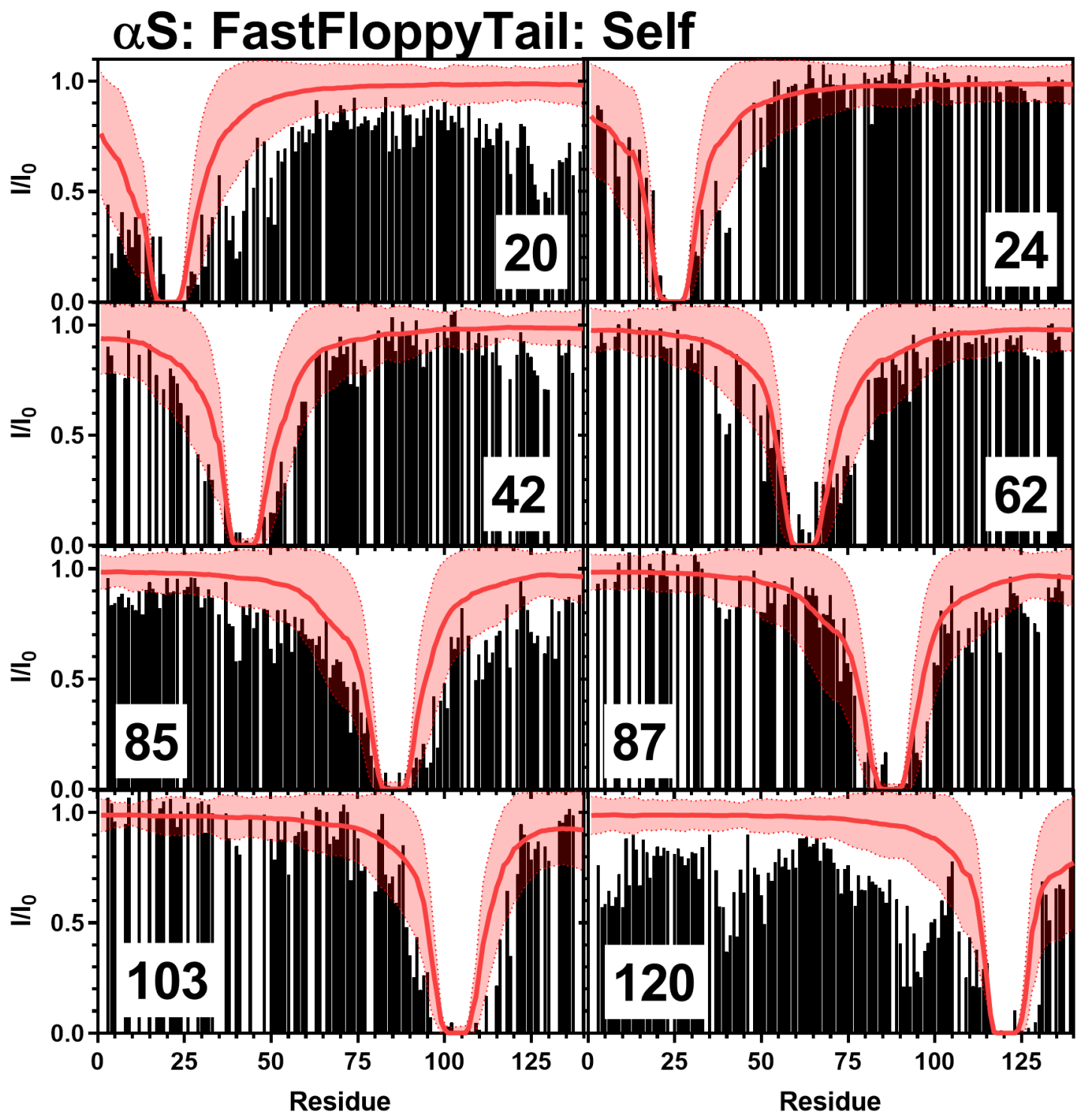

Figure S179: Comparison of Simulated PRE Values and Experimental PRE Values: Simulated PRE values from FastFloppyTail using self-reweighting (red line) overlayed on top of experimental data (black bars) from positions 20 (Top Left), 24 (Top Right), 42 (Upper Middle Left), 62 (Upper Middle Right), 85 (Lower Middle Left), 87 (Lower Middle Right), 103 (Bottom Left), 120 (Bottom Right). Experimental data for positions 20, 85, and 120 are from Sung et al. ${ }^{8}$ and data for positions $24,42,62,87$, and 103 are from Dedmon et al. ${ }^{9}$. 


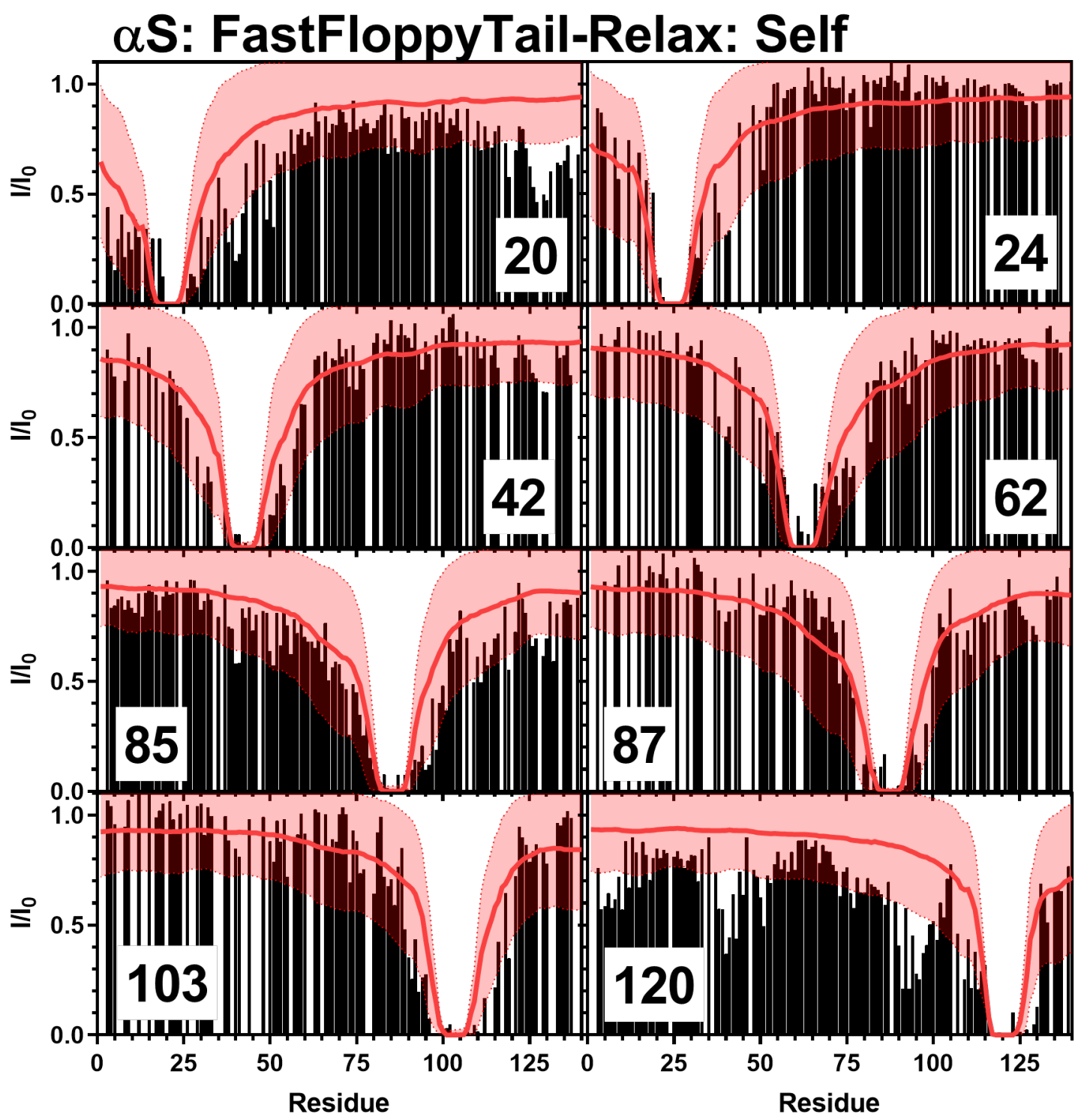

Figure S180: Comparison of Simulated PRE Values and Experimental PRE Values: Simulated PRE values from FastFloppyTail-Relax using self-reweighting (red line) overlayed on top of experimental data (black bars) from positions 20 (Top Left), 24 (Top Right), 42 (Upper Middle Left), 62 (Upper Middle Right), 85 (Lower Middle Left), 87 (Lower Middle Right), 103 (Bottom Left), 120 (Bottom Right). Experimental data for positions 20, 85, and 120 are from Sung et al. ${ }^{8}$ and data for positions $24,42,62,87$, and 103 are from Dedmon et al. ${ }^{9}$. 


\section{Comparisons of DSH3 Data}
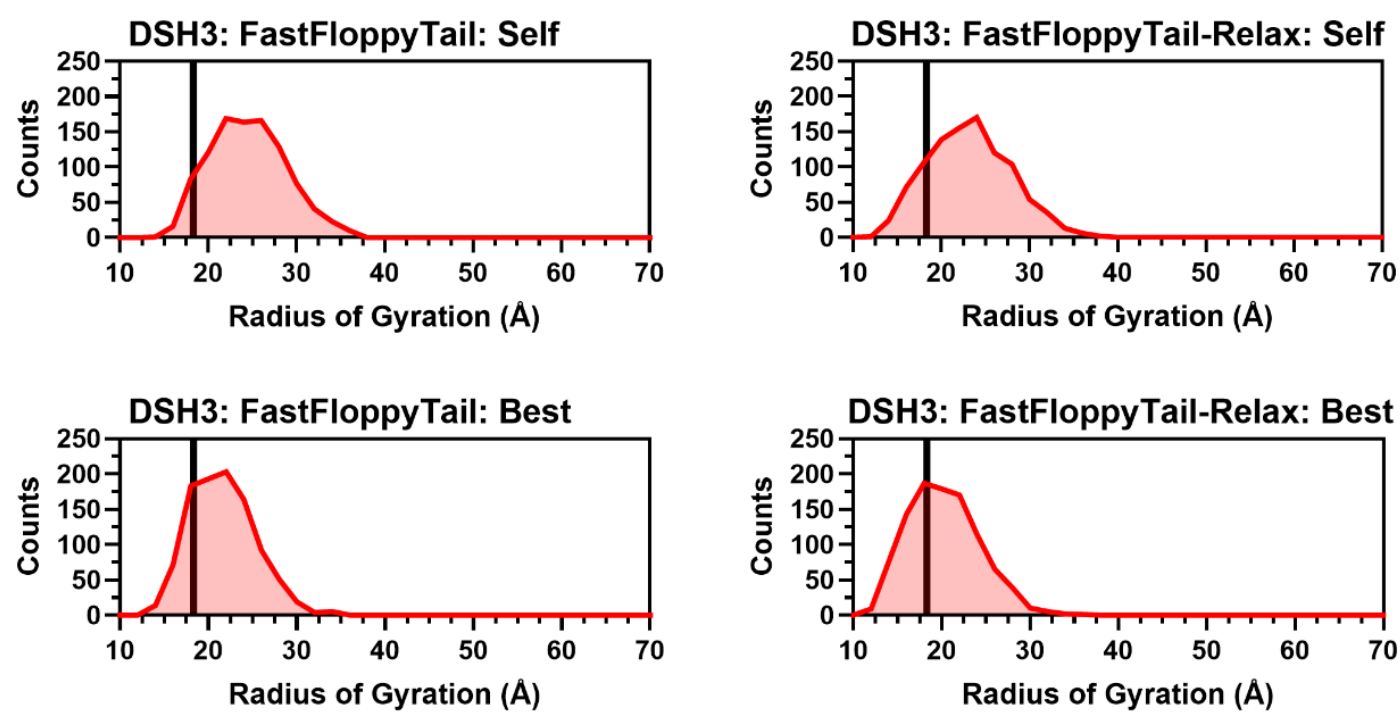

Figure S190: Histograms of Radii of Gyration for DSH3. Histograms of the radius of gyration from self (Top Left) and best (Top Right) reweighting in FastFloppyTail and self (Bottom Left) and best (Bottom Right) reweighting in FastFloppyTail-Relax compared to experimental values reported by Marsh et al. (black) ${ }^{31}$.
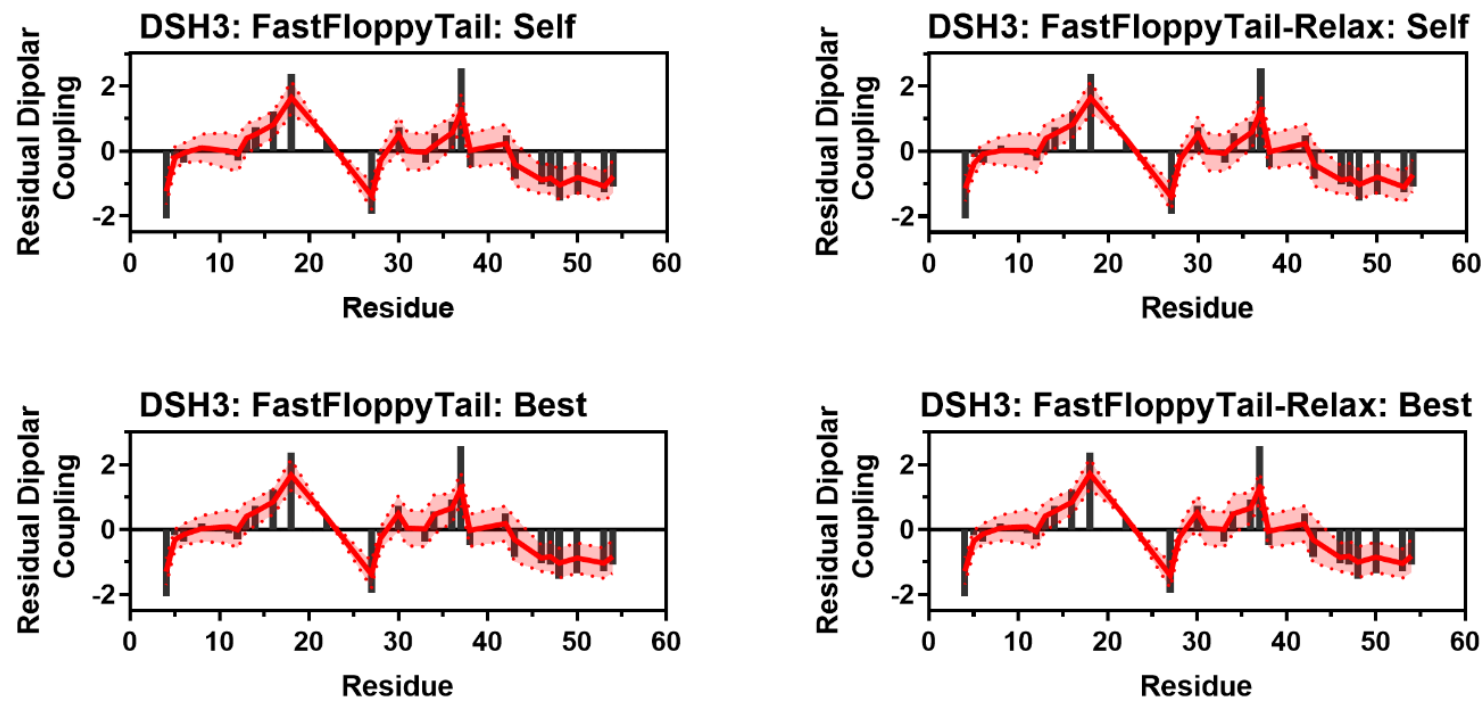

Figure S191: Comparison of Simulated and Experimental Residual Dipolar Coupling Data. Simulated RDC values (red line) from self (Top Left) and best (Top Right) reweighting in FastFloppyTail and self (Bottom Left) and best (Bottom Right) reweighting in FastFloppyTailRelax overlayed on experimental data (black bars) from Marsh et al. ${ }^{31}$. 

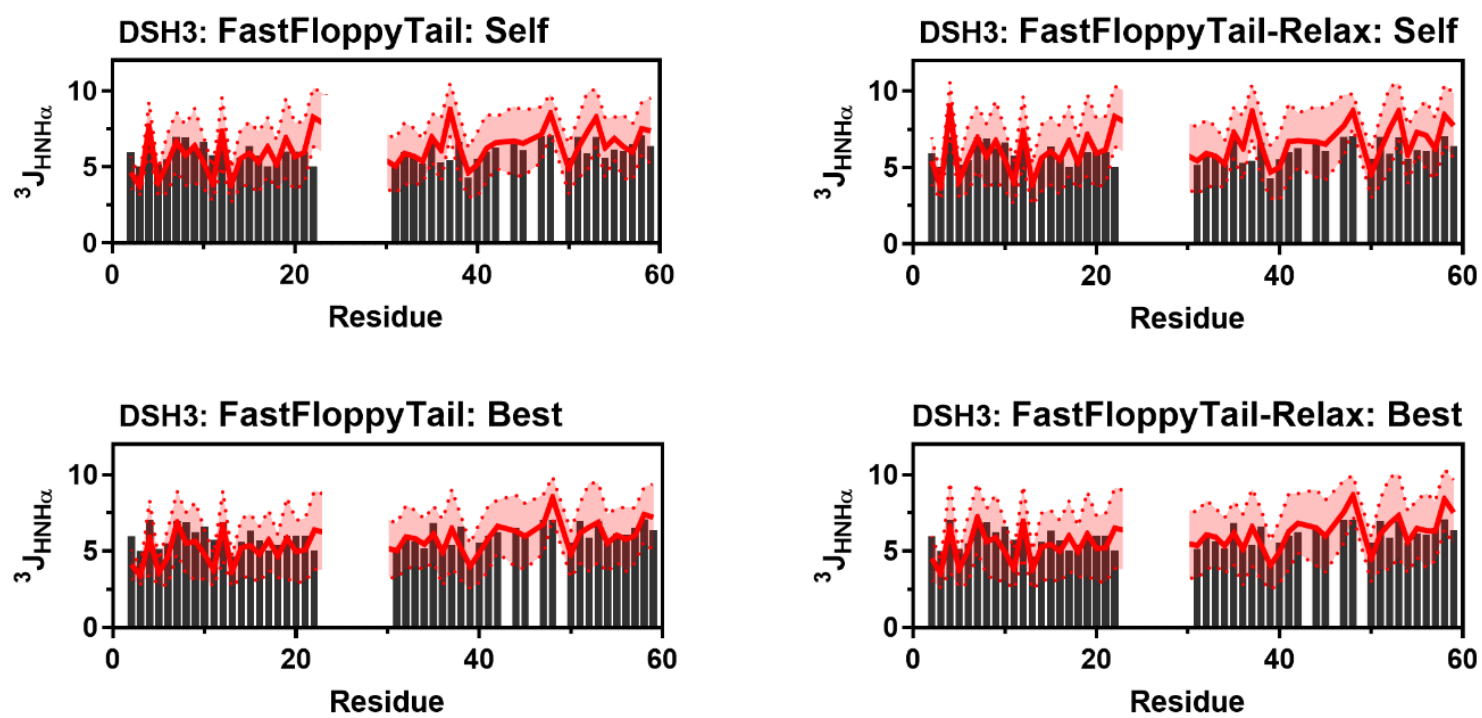

Figure S192: Comparison of Simulated and Experimental J-Coupling Data. Simulated ${ }^{3} \mathrm{~J}_{\mathrm{HNH} \alpha}$ values (red lines) from self (Top Left) and best (Top Right) reweighting in FastFloppyTail and self (Bottom Left) and best (Bottom Right) reweighting in FastFloppyTail-Relax compared to experimental values from Zhang et al. (black bars) ${ }^{37}$. 

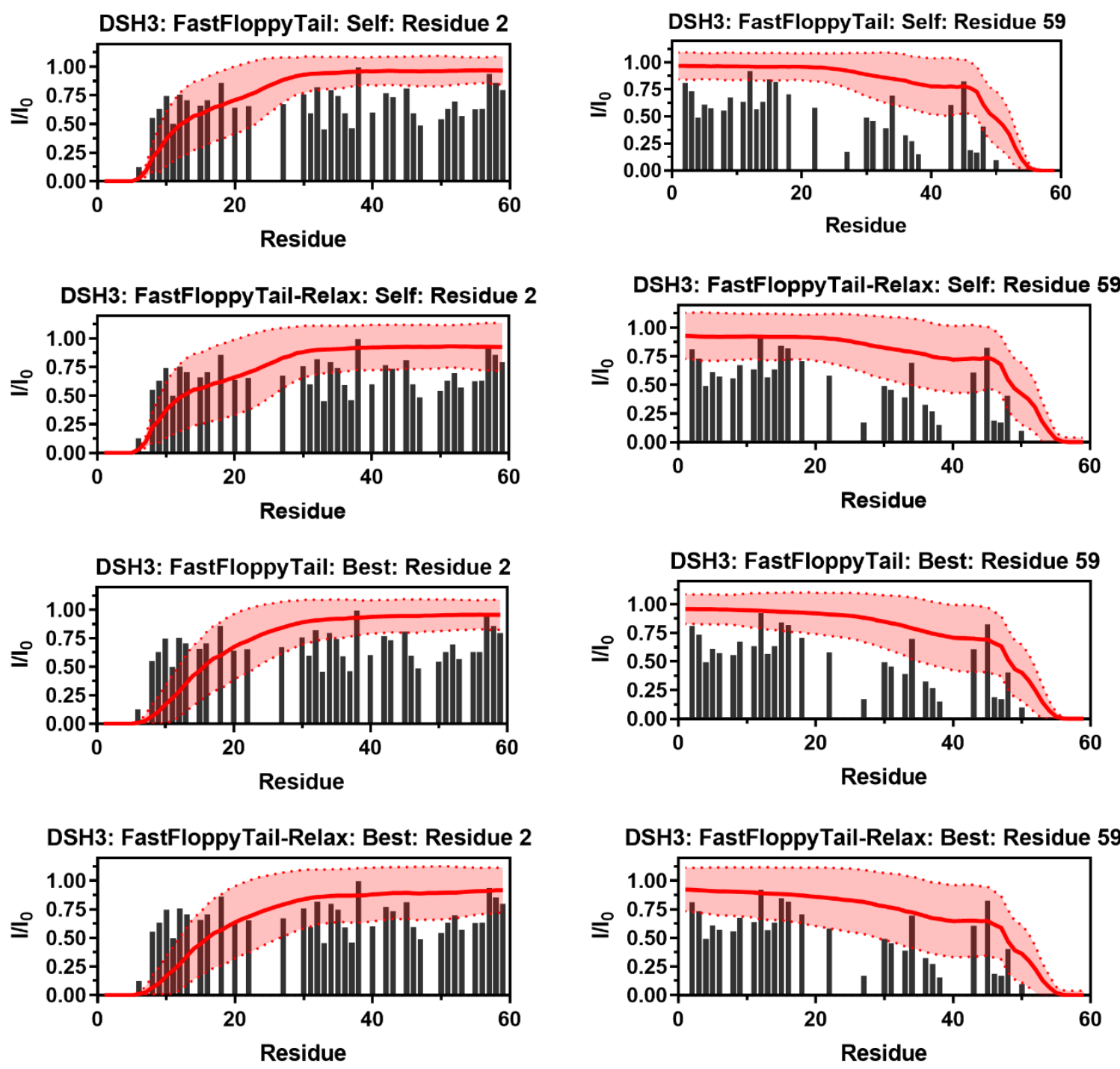

Figure S193: Comparison of Simulated PRE Values and Experimental PRE Values: Simulated PRE values (red lines) from FastFloppyTail (Row 1) and FastFloppyTail-Relax (Row 2) using self-reweighting and FastFloppyTail (Row 3) and FastFloppyTail-Relax (Row 4) using bestreweighting overlayed on top of experimental data (black bars) from positions 2 (Left) and 59 (Right) from Marsh et al. (black) ${ }^{31}$. 


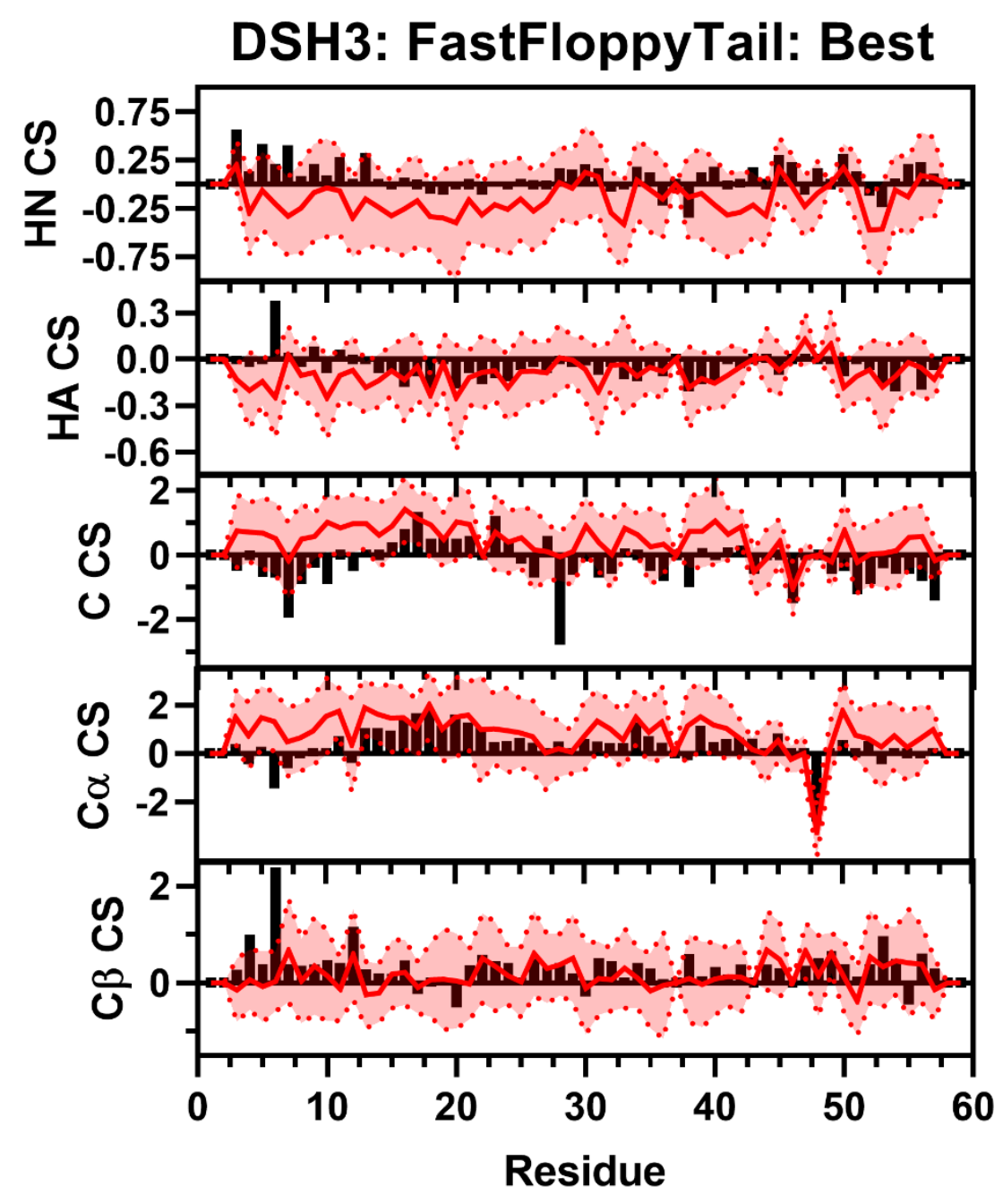

Figure S194: Comparison of Simulated and Experimental NMR Secondary Chemical Shift Data for DSH3. Simulated Chemical Shift values from the FastFloppyTail ensemble using bestreweighted fragment selection (red) overlayed on experimental data (black bars) of HN (Row 1), HA (Row 2), C (Row 3), C $\alpha$ (Row 4), and C $\beta$ (Row 5) chemical shifts from Zhang et al. ${ }^{37}$ Neighbor corrected random coil chemical shift values from Sparta+ have been subtracted from both simulated and experimental chemical shifts to generate secondary chemical shift data ${ }^{11}$. 


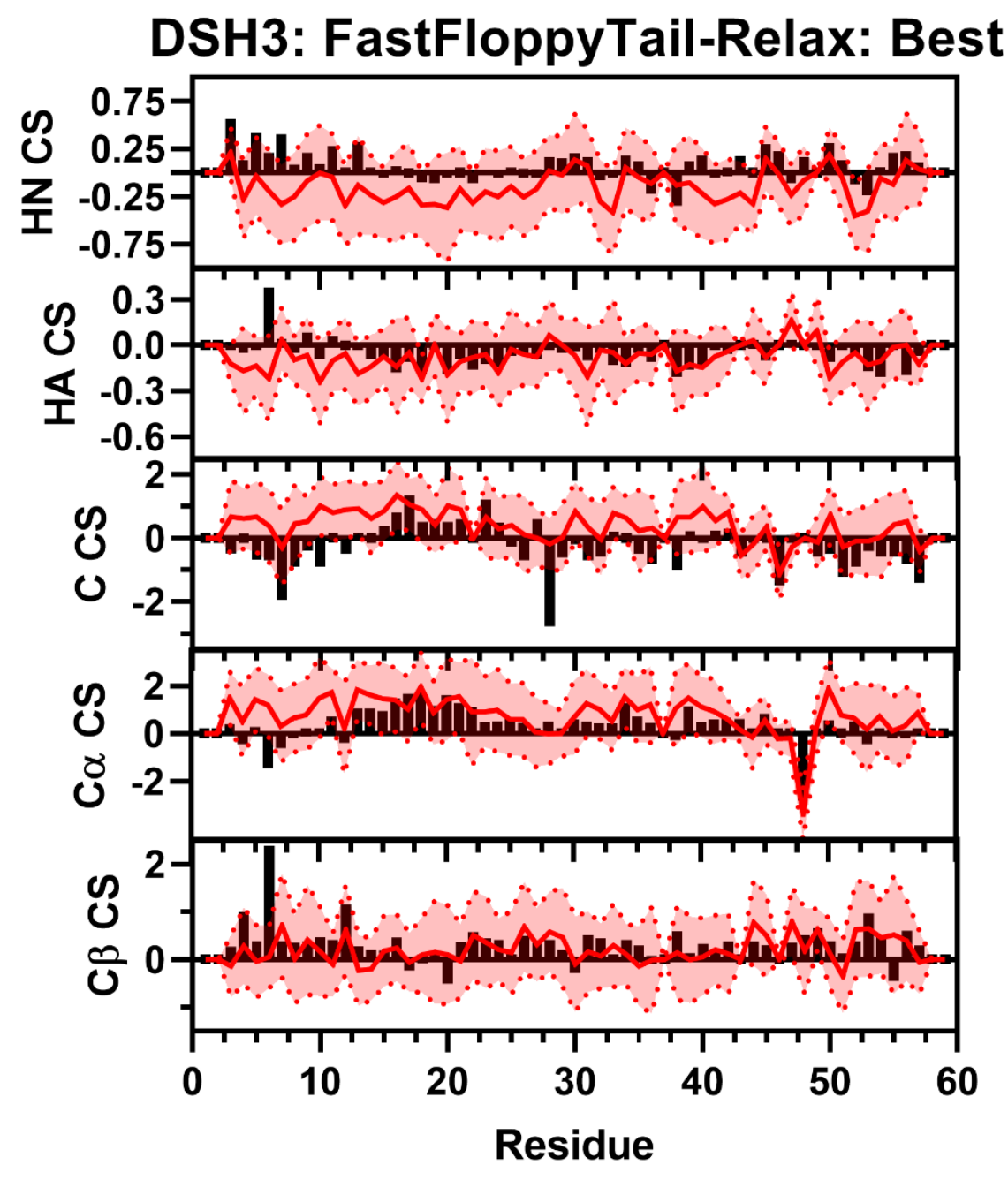

Figure S195: Comparison of Simulated and Experimental NMR Secondary Chemical Shift Data for DSH3. Simulated Chemical Shift values from the FastFloppyTail-Relax ensemble using bestreweighted fragment selection (red) overlayed on experimental data (black bars) of HN (Row 1), HA (Row 2), C (Row 3), $\mathrm{C} \alpha$ (Row 4), and C $\beta$ (Row 5) chemical shifts from Zhang et al. ${ }^{37}$ Neighbor corrected random coil chemical shift values from SPARTA+ have been subtracted from both simulated and experimental chemical shifts to generate secondary chemical shift data ${ }^{11}$. 


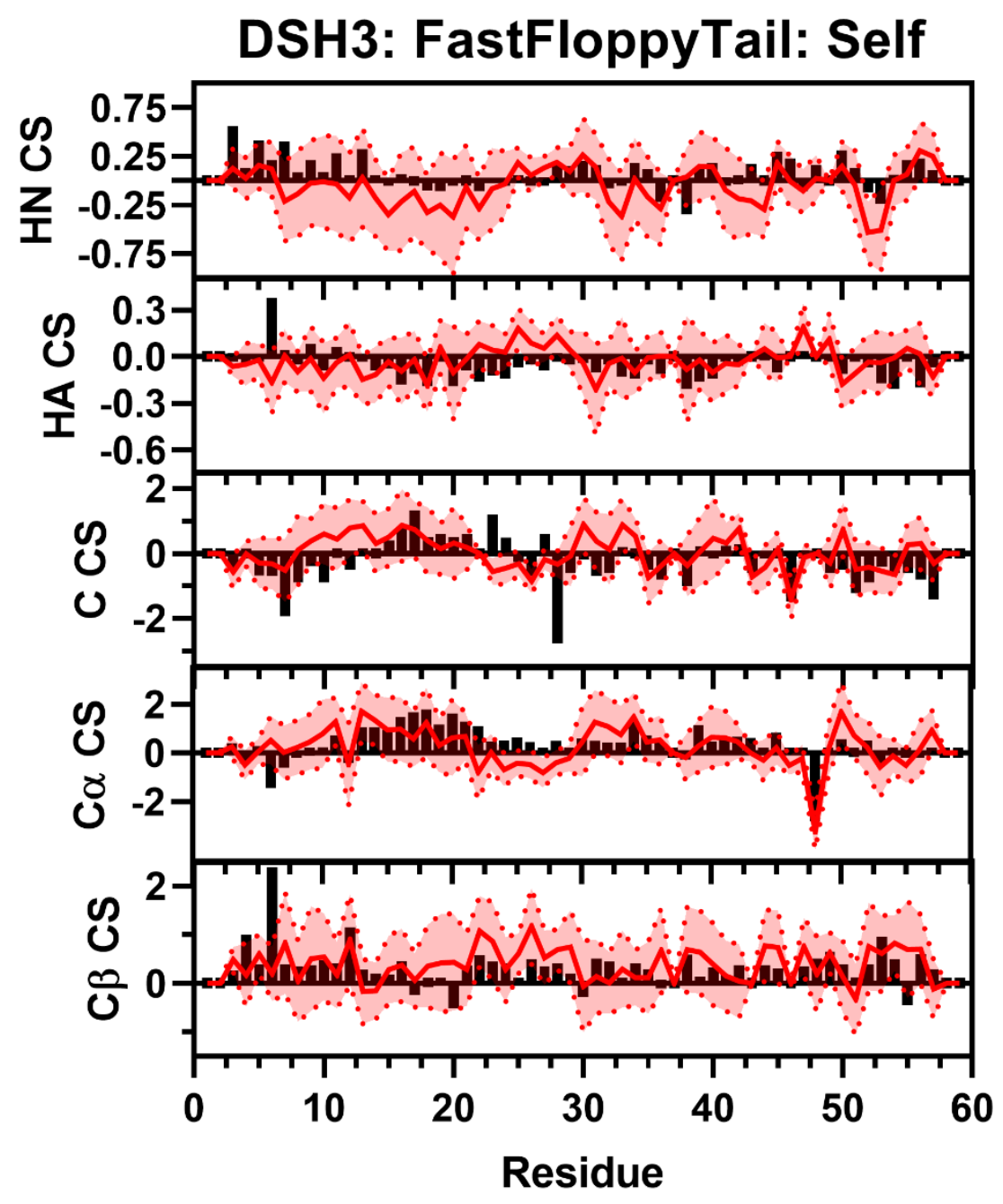

Figure S196: Comparison of Simulated and Experimental NMR Secondary Chemical Shift Data for DSH3. Simulated Chemical Shift values from the FastFloppyTail ensemble using selfreweighted fragment selection (red) overlayed on experimental data (black bars) of HN (Row 1), HA (Row 2), C (Row 3), $\mathrm{C} \alpha$ (Row 4), and $\mathrm{C} \beta$ (Row 5) chemical shifts from Zhang et al. ${ }^{37}$ Neighbor corrected random coil chemical shift values from Sparta + have been subtracted from both simulated and experimental chemical shifts to generate secondary chemical shift data ${ }^{11}$. 


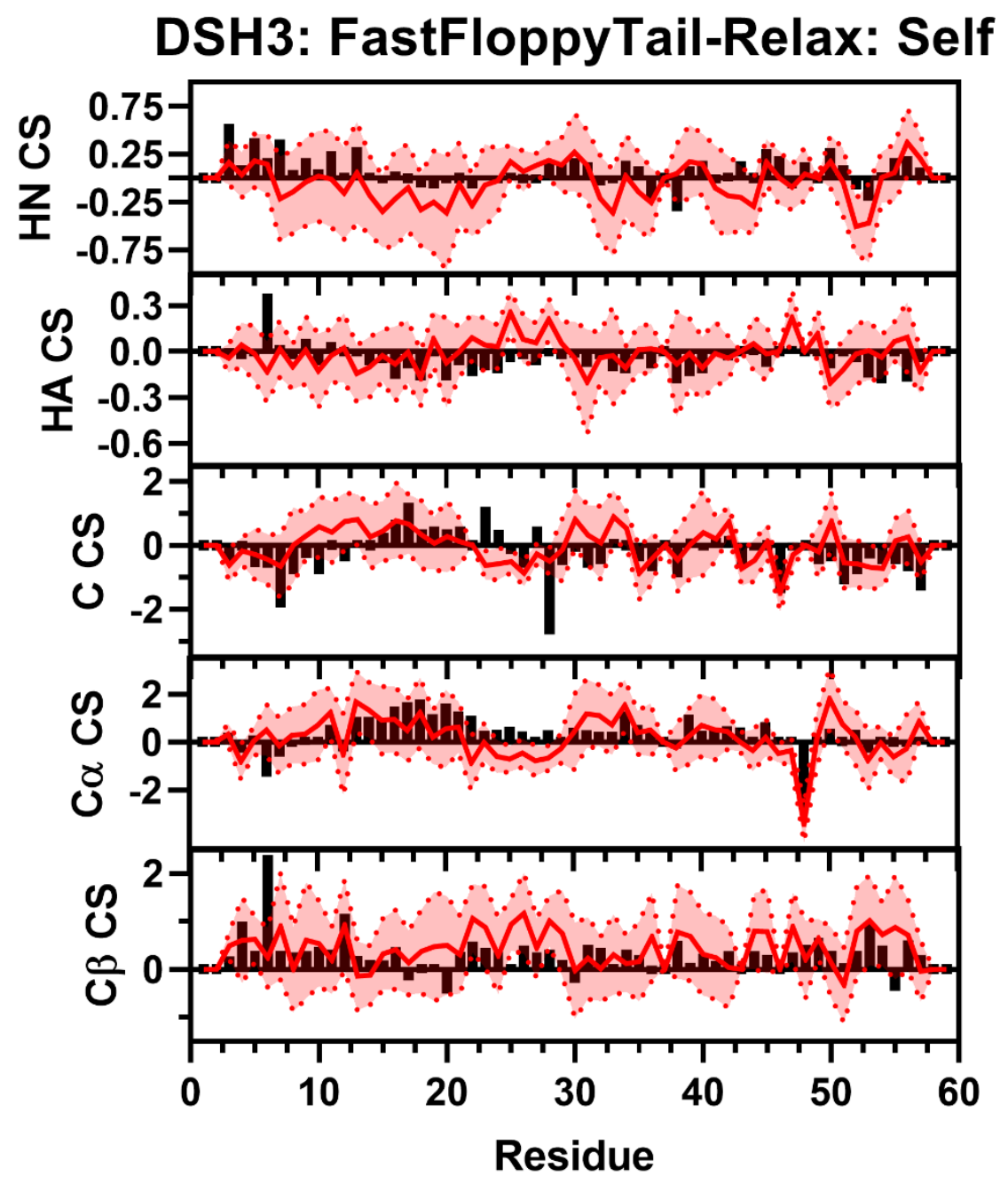

Figure S197: Comparison of Simulated and Experimental NMR Secondary Chemical Shift Data for DSH3. Simulated Chemical Shift values from the FastFloppyTail-Relax ensemble using selfreweighted fragment selection (red) overlayed on experimental data (black bars) of HN (Row 1), HA (Row 2), C (Row 3), $\mathrm{C} \alpha$ (Row 4), and $\mathrm{C} \beta$ (Row 5) chemical shifts from Zhang et al. ${ }^{37}$ Neighbor corrected random coil chemical shift values from Sparta + have been subtracted from both simulated and experimental chemical shifts to generate secondary chemical shift data ${ }^{11}$. 


\section{Comparisons of NTAL Data}
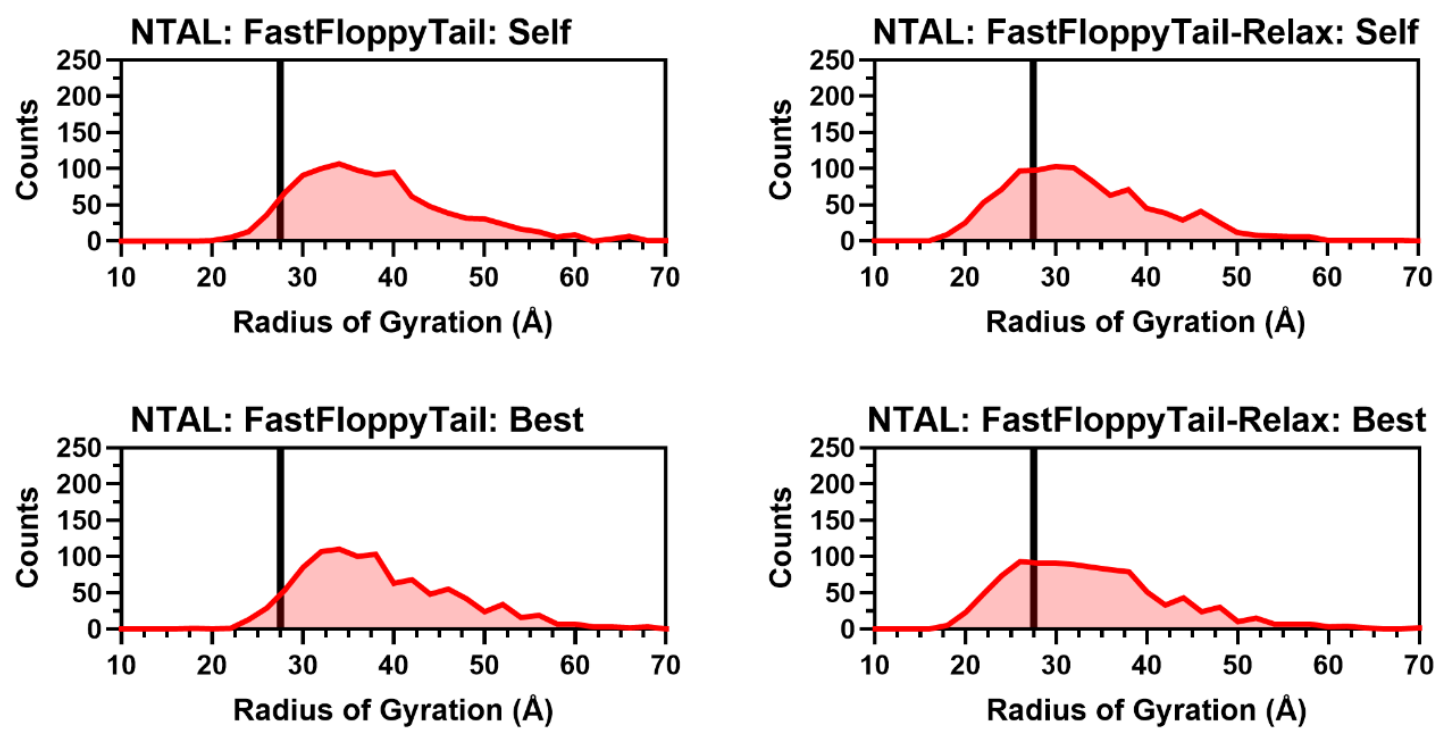

Figure S198: Histograms of Radii of Gyration for DSH3. Histograms of the radius of gyration from self (Top Left) and best (Top Right) reweighting in FastFloppyTail and self (Bottom Left) and best (Bottom Right) reweighting in FastFloppyTail-Relax compared to experimental values reported by Jensen et al. (black) ${ }^{32}$.
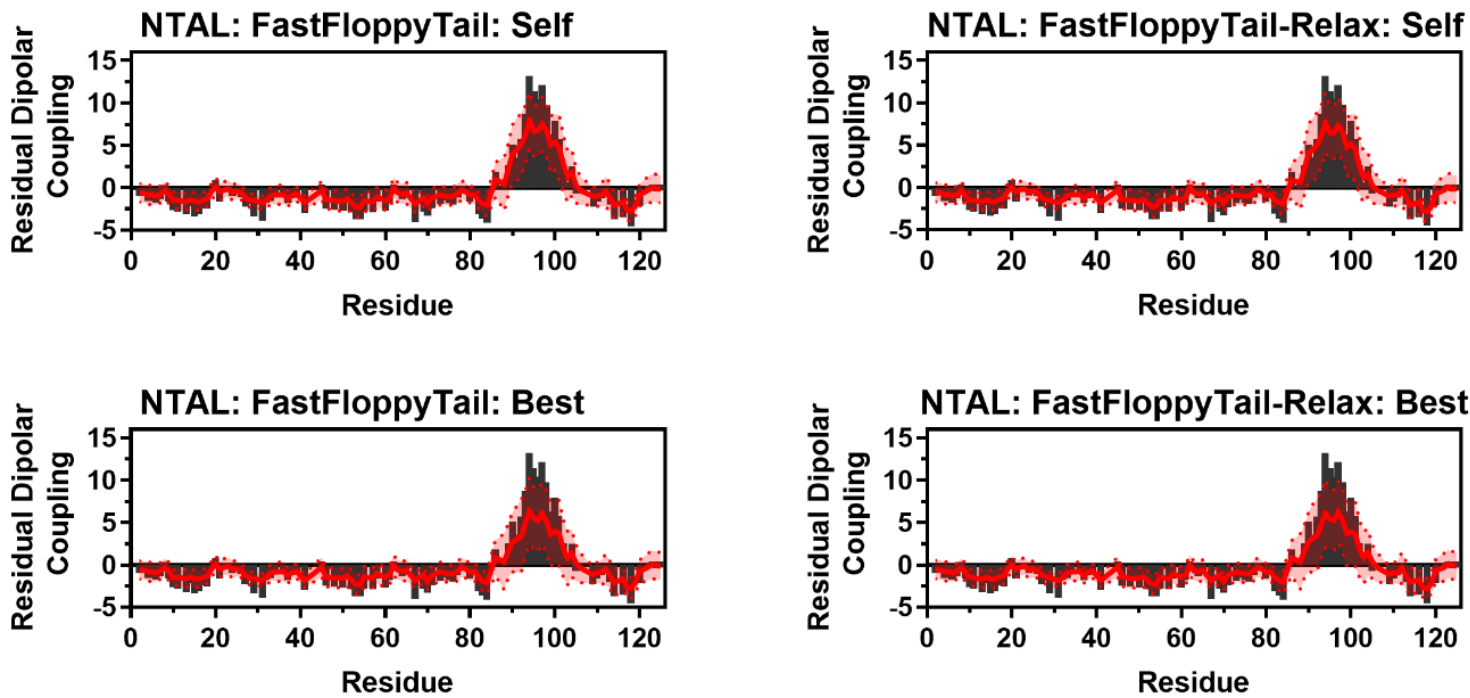

Figure S199: Comparison of Simulated and Experimental Residual Dipolar Coupling Data. Simulated RDC values (red line) from self (Top Left) and best (Top Right) reweighting in FastFloppyTail and self (Bottom Left) and best (Bottom Right) reweighting in FastFloppyTailRelax overlayed on experimental data (black bars) from Jensen et al. ${ }^{32}$. 


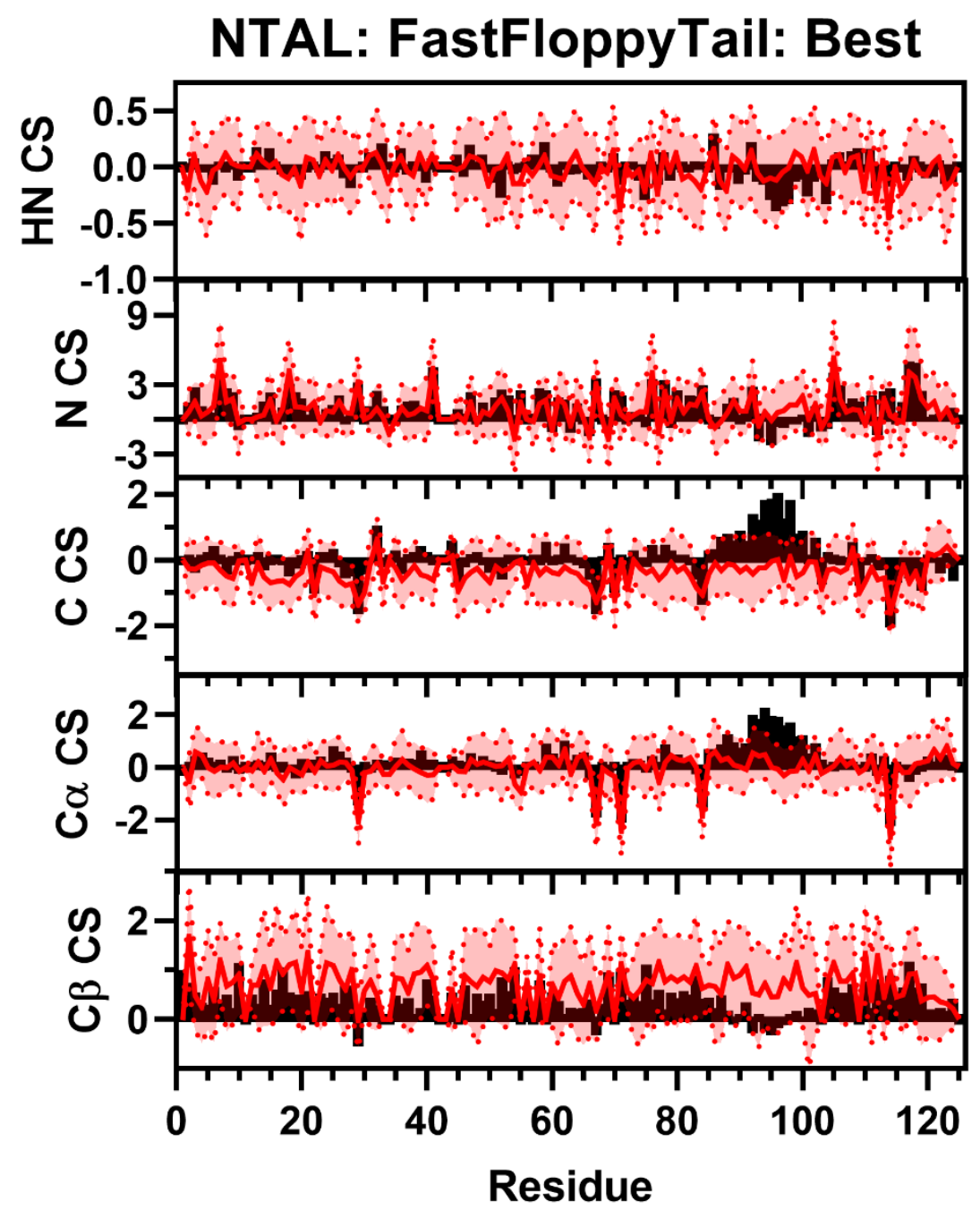

Figure S200: Comparison of Simulated and Experimental NMR Secondary Chemical Shift Data for NTAL. Simulated Chemical Shift values from the FastFloppyTail ensemble using bestreweighted fragment selection (red) overlayed on experimental data (black bars) of HN (Row 1), HA (Row 2), C (Row 3), C $\alpha$ (Row 4), and C $\beta$ (Row 5) chemical shifts from Jensen et. al. ${ }^{32}$ Neighbor corrected random coil chemical shift values from SPARTA+ have been subtracted from both simulated and experimental chemical shifts to generate secondary chemical shift data ${ }^{11}$. 


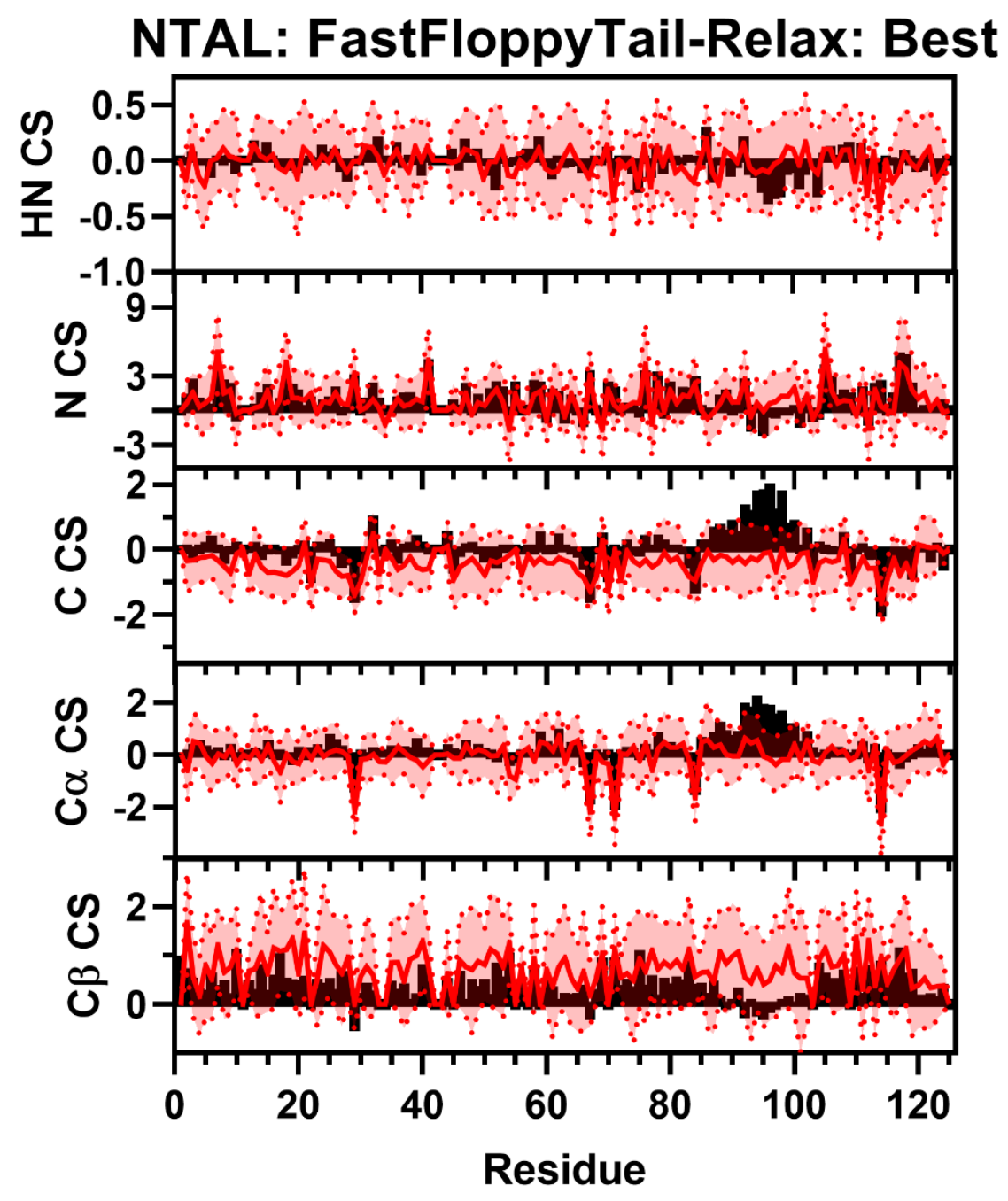

Figure S201: Comparison of Simulated and Experimental NMR Secondary Chemical Shift Data for NTAL. Simulated Chemical Shift values from the FastFloppyTail-Relax ensemble using bestreweighted fragment selection (red) overlayed on experimental data (black bars) of HN (Row 1), HA (Row 2), C (Row 3), C $\alpha$ (Row 4), and C $\beta$ (Row 5) chemical shifts from Jensen et al. ${ }^{32}$ Neighbor corrected random coil chemical shift values from SPARTA+ have been subtracted from both simulated and experimental chemical shifts to generate secondary chemical shift data ${ }^{11}$. 


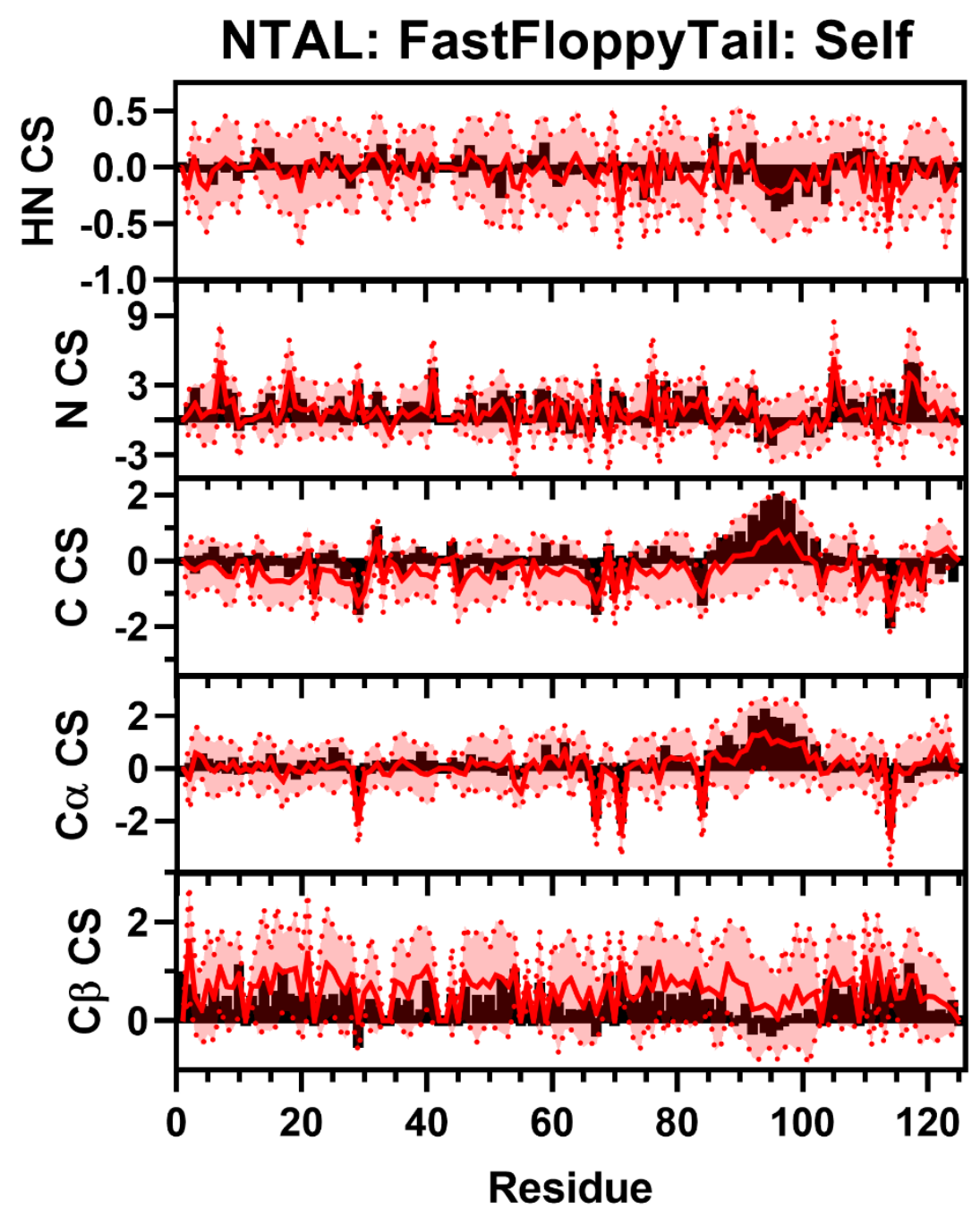

Figure S202: Comparison of Simulated and Experimental NMR Secondary Chemical Shift Data for NTAL. Simulated Chemical Shift values from the FastFloppyTail ensemble using selfreweighted fragment selection (red) overlayed on experimental data (black bars) of $\mathrm{HN}$ (Row 1), HA (Row 2), C (Row 3), C $\alpha$ (Row 4), and C $\beta$ (Row 5) chemical shifts from Jensen et al. ${ }^{32}$ Neighbor corrected random coil chemical shift values from Sparta + have been subtracted from both simulated and experimental chemical shifts to generate secondary chemical shift data ${ }^{11}$. 


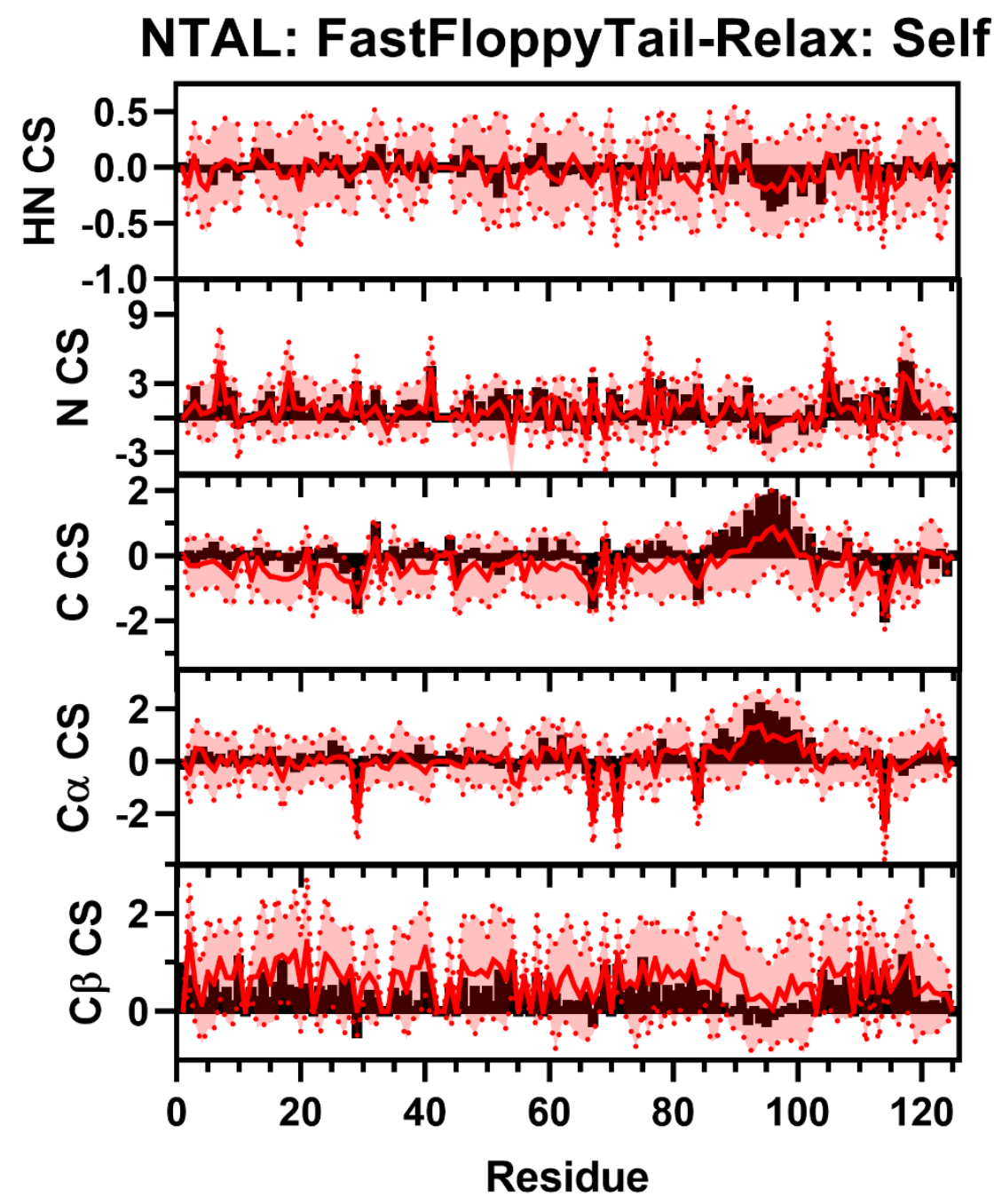

Figure S203: Comparison of Simulated and Experimental NMR Secondary Chemical Shift Data for NTAL. Simulated Chemical Shift values from the FastFloppyTail-Relax ensemble using selfreweighted fragment selection (red) overlayed on experimental data (black bars) of HN (Row 1), HA (Row 2), C (Row 3), C $\alpha$ (Row 4), and C $\beta$ (Row 5) chemical shifts from Jensen et al. ${ }^{32}$ Neighbor corrected random coil chemical shift values from Sparta + have been subtracted from both simulated and experimental chemical shifts to generate secondary chemical shift data ${ }^{11}$. 


\section{Comparisons of PAAA Data}
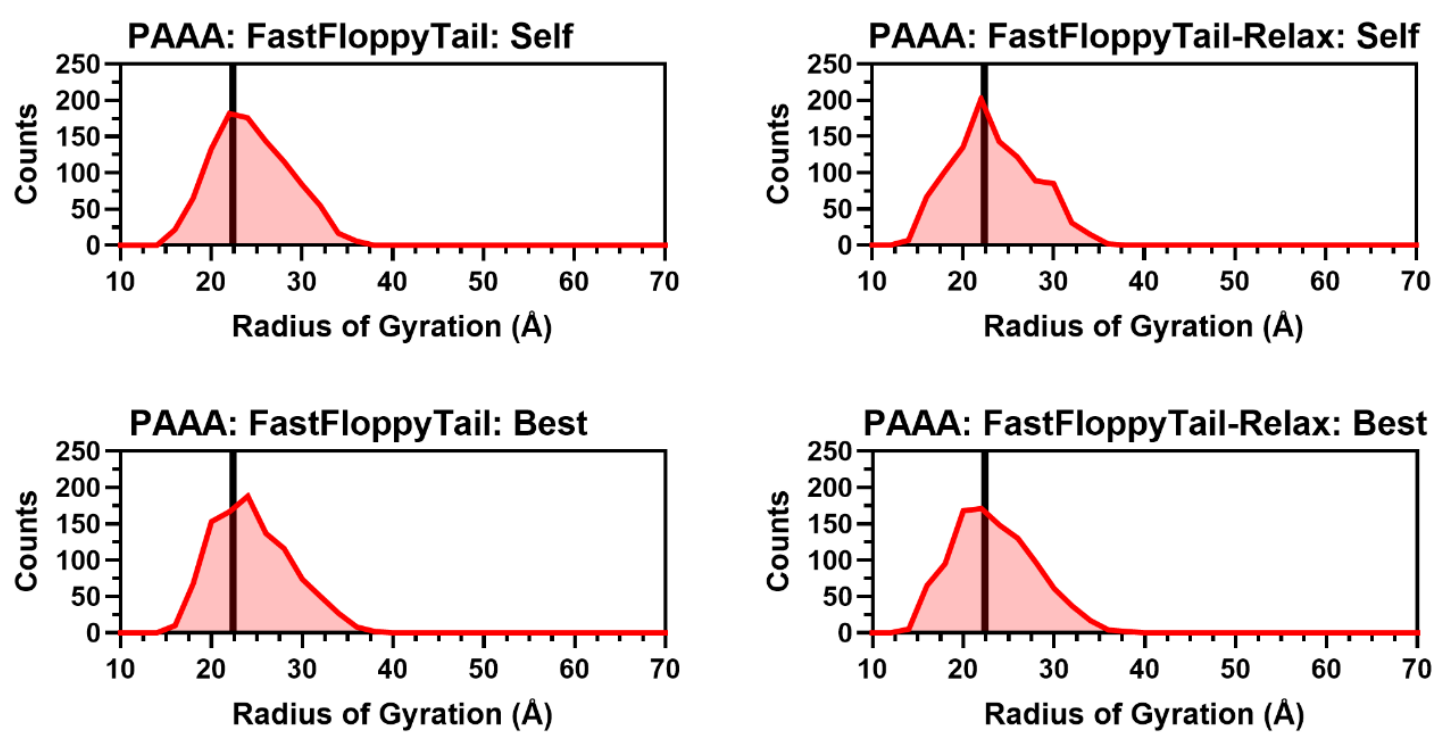

Figure S204: Histograms of Radii of Gyration for PAAA. Histograms of the radius of gyration from self (Top Left) and best (Top Right) reweighting in FastFloppyTail and self (Bottom Left) and best (Bottom Right) reweighting in FastFloppyTail-Relax compared to experimental values from NMR (black) ${ }^{33}$.
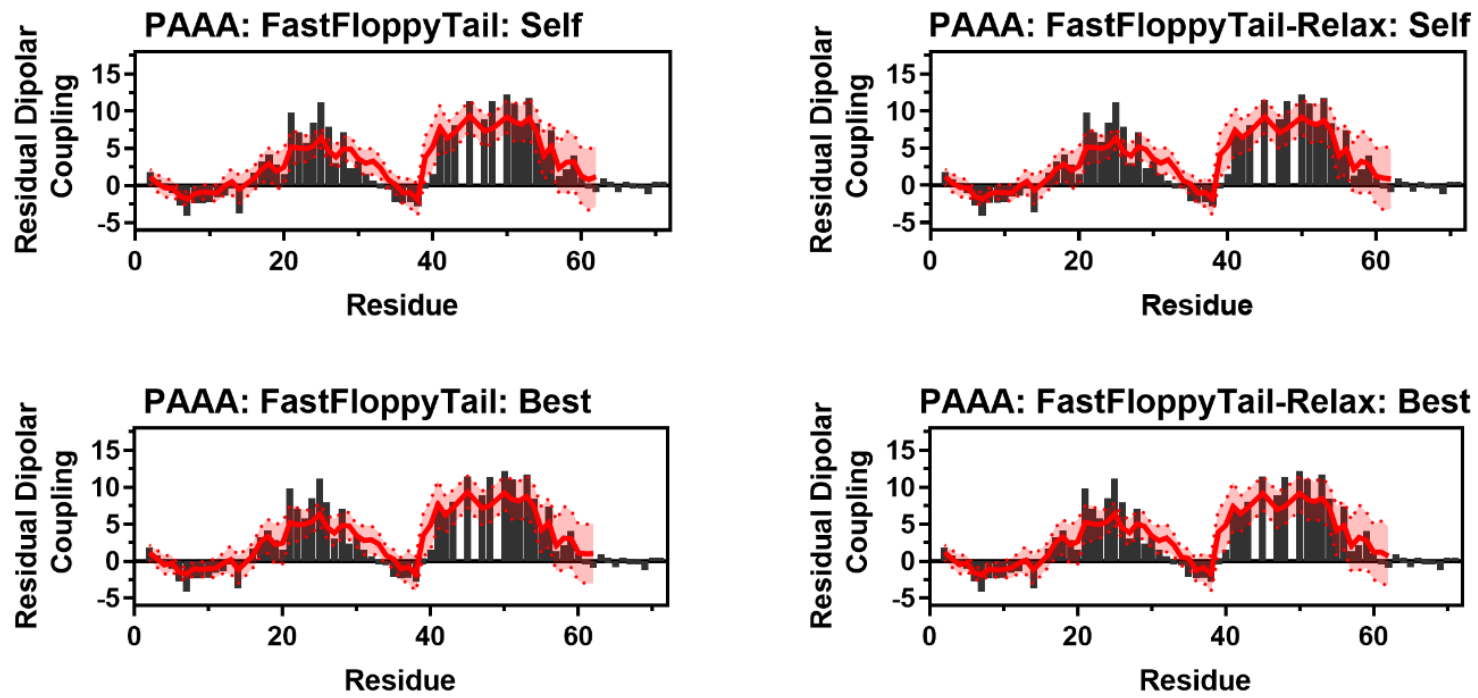

Figure S205: Comparison of Simulated and Experimental Residual Dipolar Coupling Data. Simulated RDC values (red line) from self (Top Left) and best (Top Right) reweighting in FastFloppyTail and self (Bottom Left) and best (Bottom Right) reweighting in FastFloppyTailRelax overlayed on experimental data (black bars) from Streckx et al. ${ }^{33}$. 


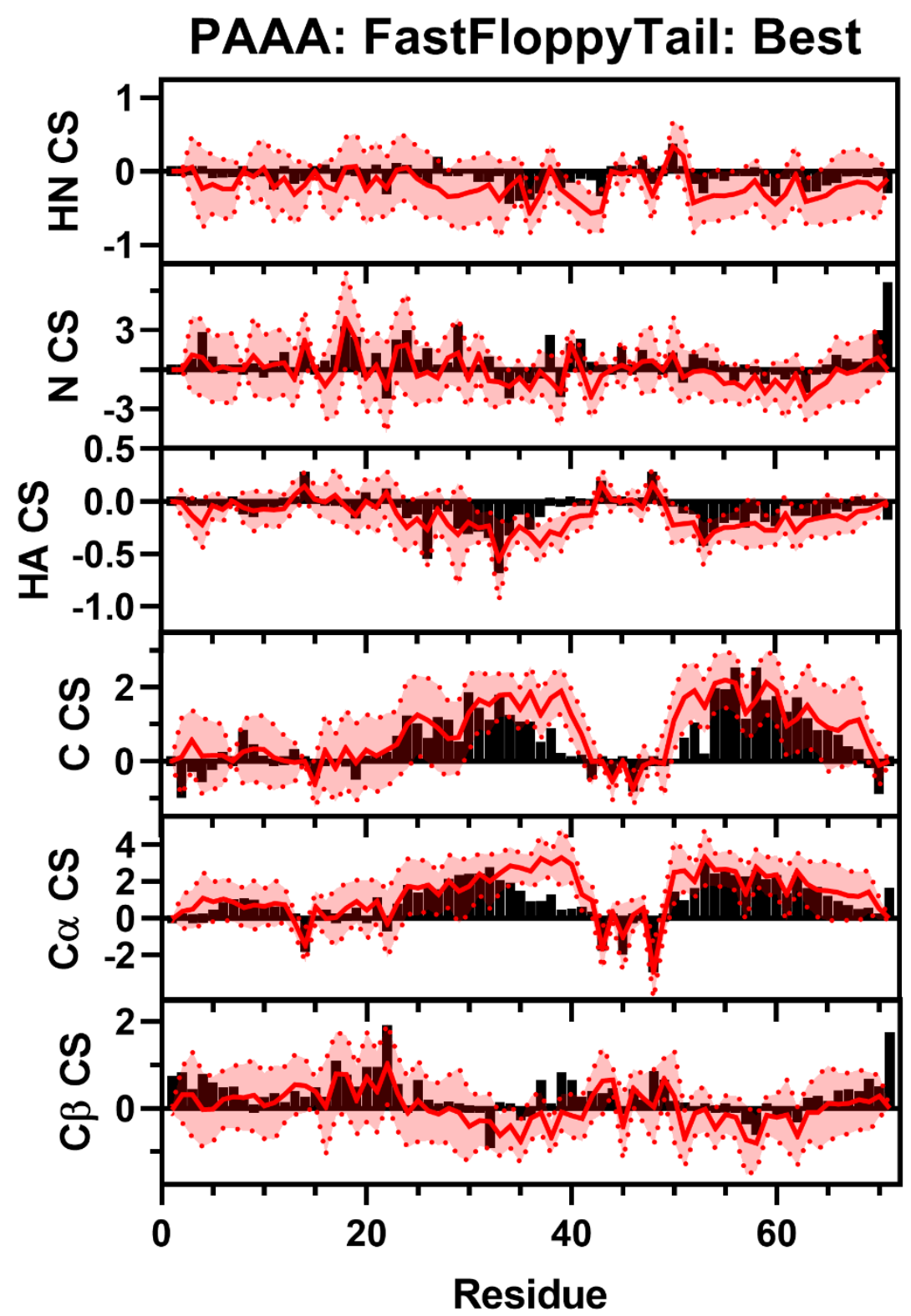

Figure S206: Comparison of Simulated and Experimental NMR Secondary Chemical Shift Data for NTAL. Simulated Chemical Shift values from the FastFloppyTail ensemble using bestreweighted fragment selection (red) overlayed on experimental data (black bars) of HN (Row 1), $\mathrm{N}$ (Row 2), HA (Row 3), C, (Row 4), C $\alpha$ (Row 5), and C $\beta$ (Row 6) chemical shifts from Streckx et al. ${ }^{33}$ Neighbor corrected random coil chemical shift values from Sparta + have been subtracted from both simulated and experimental chemical shifts to generate secondary chemical shift data 11 . 


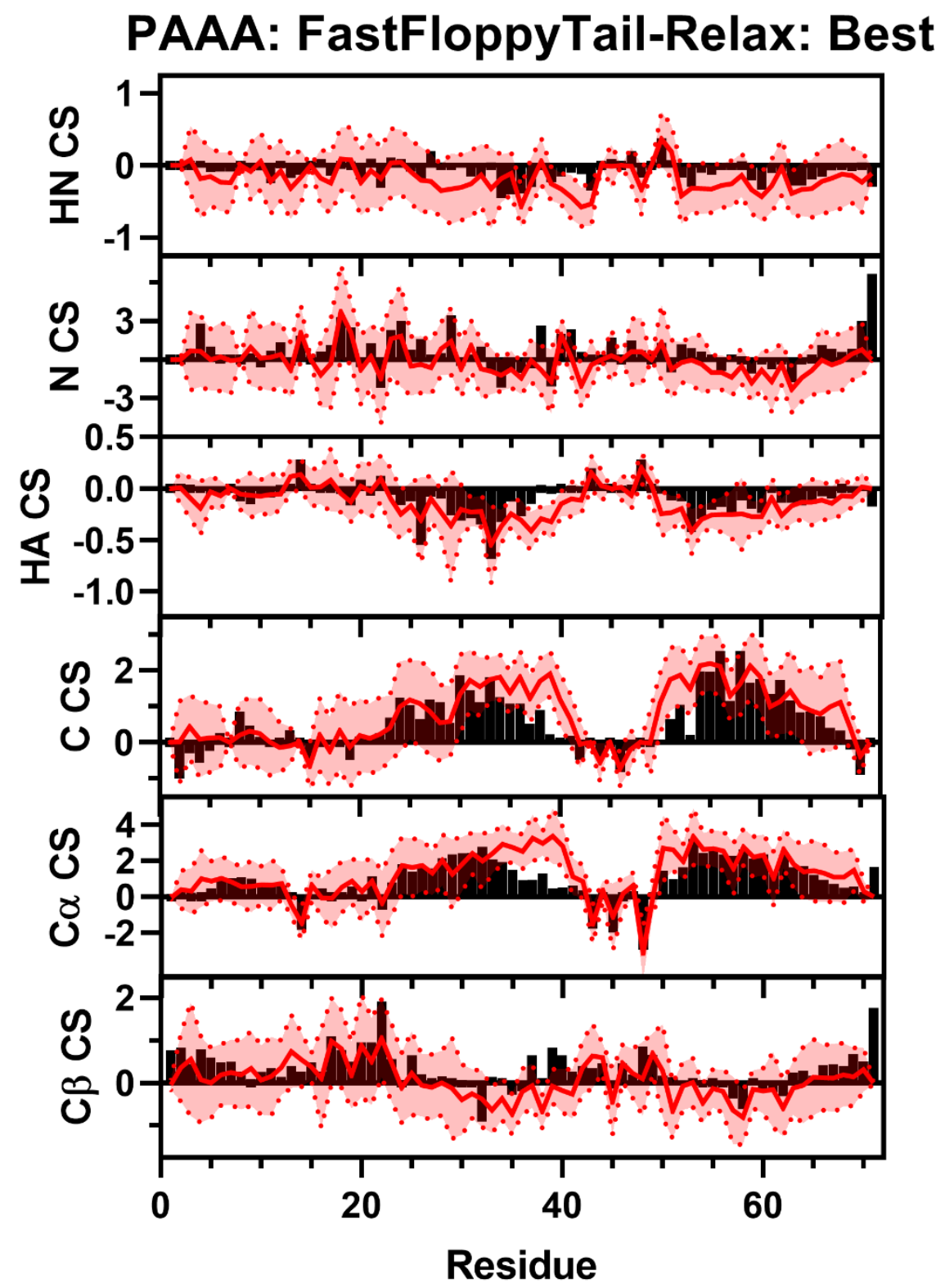

Figure S207: Comparison of Simulated and Experimental NMR Secondary Chemical Shift Data for NTAL. Simulated Chemical Shift values from the FastFloppyTail-Relax ensemble using bestreweighted fragment selection (red) overlayed on experimental data (black bars) of HN (Row 1), $\mathrm{N}$ (Row 2), HA (Row 3), C, (Row 4), C $\alpha$ (Row 5), and C $\beta$ (Row 6) chemical shifts from Streckx et al. ${ }^{33}$ Neighbor corrected random coil chemical shift values from SPARTA+ have been subtracted from both simulated and experimental chemical shifts to generate secondary chemical shift data ${ }^{11}$. 


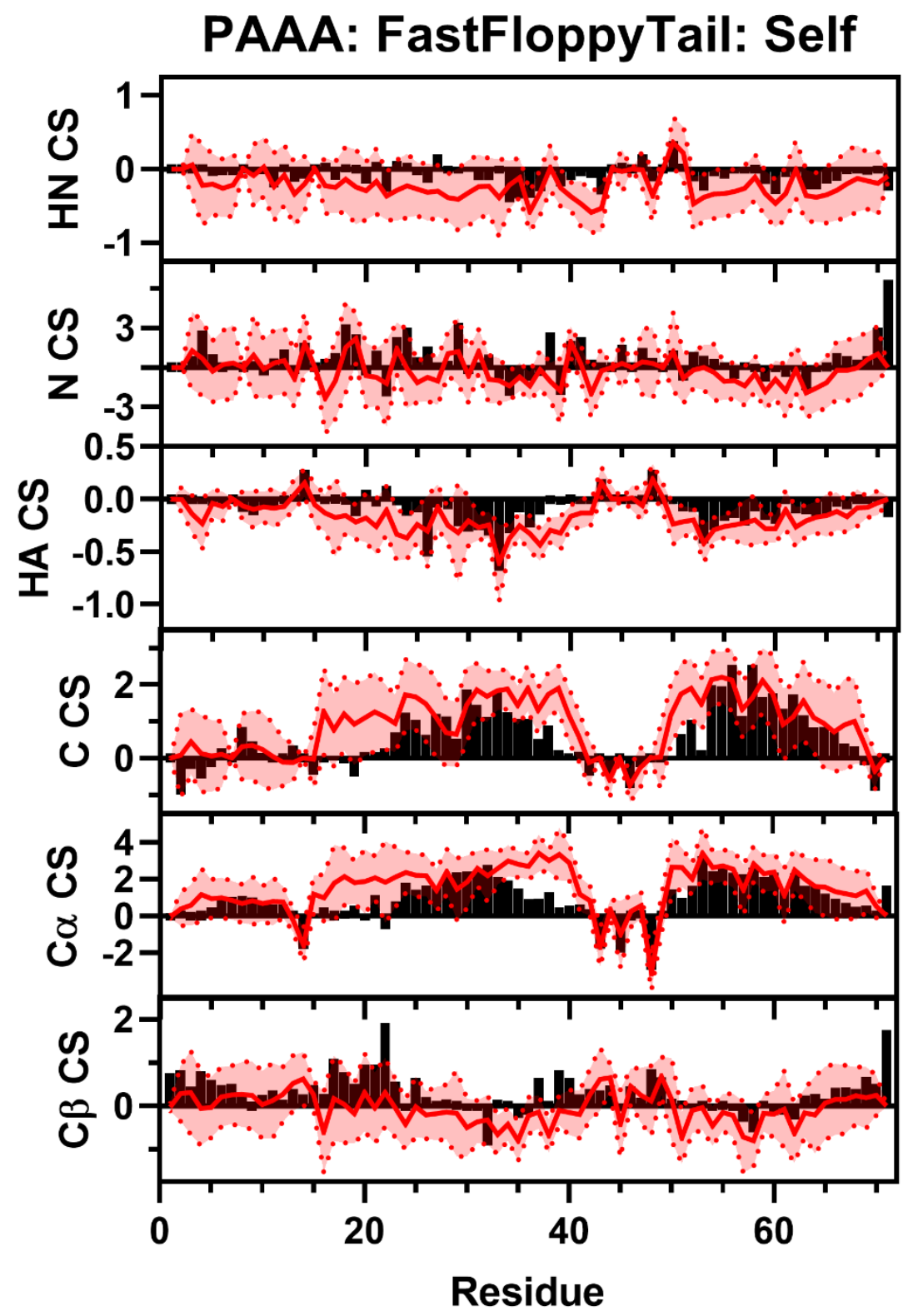

Figure S208: Comparison of Simulated and Experimental NMR Secondary Chemical Shift Data for NTAL. Simulated Chemical Shift values from the FastFloppyTail ensemble using selfreweighted fragment selection (red) overlayed on experimental data (black bars) of HN (Row 1), N (Row 2), HA (Row 3), C, (Row 4), C $\alpha$ (Row 5), and C $\beta$ (Row 6) chemical shifts from Streckx et al. ${ }^{33}$ Neighbor corrected random coil chemical shift values from SPARTA+ have been subtracted from both simulated and experimental chemical shifts to generate secondary chemical shift data ${ }^{11}$. 


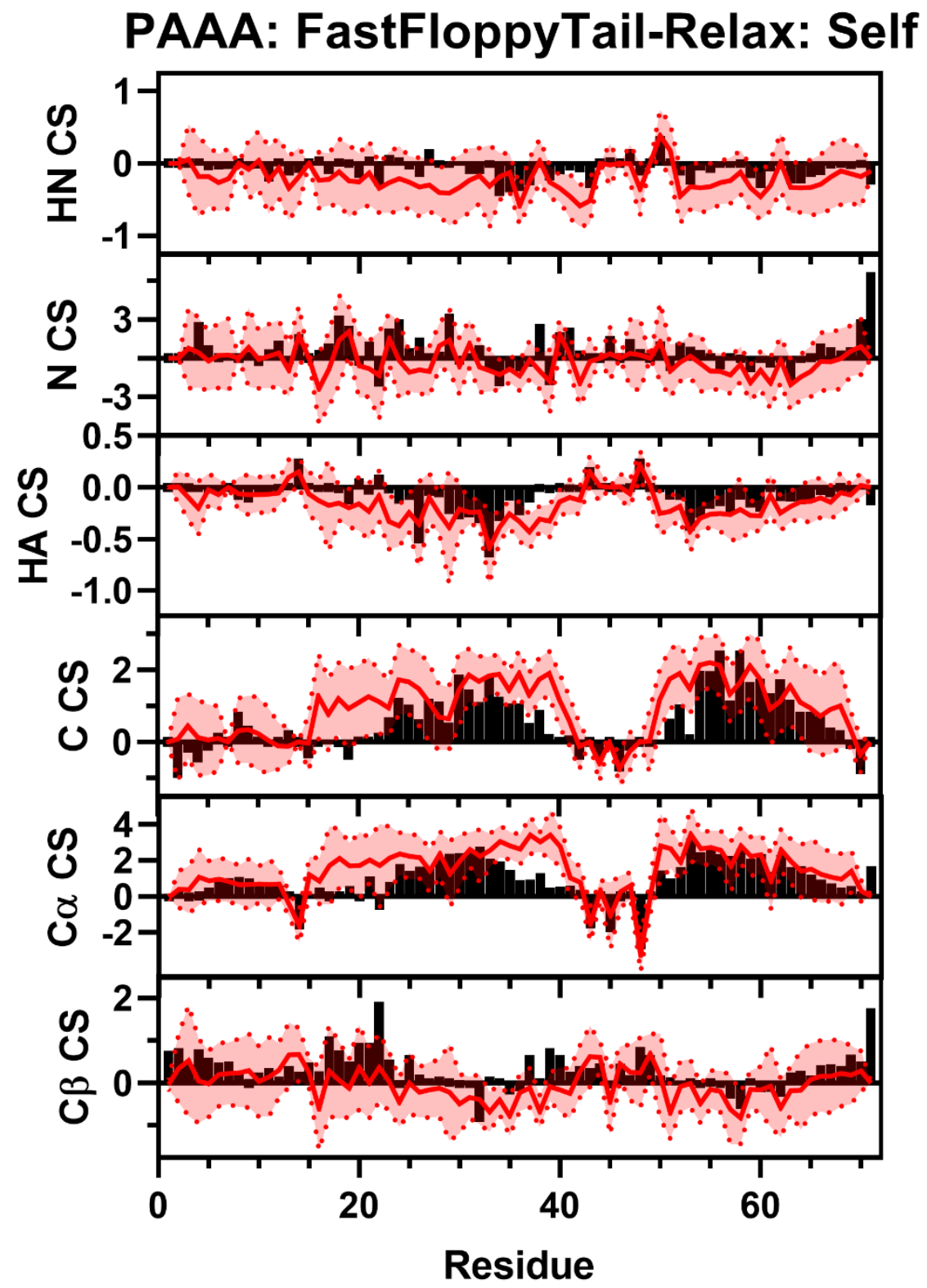

Figure S209: Comparison of Simulated and Experimental NMR Secondary Chemical Shift Data for NTAL. Simulated Chemical Shift values from the FastFloppyTail-Relax ensemble using selfreweighted fragment selection (red) overlayed on experimental data (black bars) of HN (Row 1), $\mathrm{N}$ (Row 2), HA (Row 3), C, (Row 4), C $\alpha$ (Row 5), and C $\beta$ (Row 6) chemical shifts from Streckx et al. ${ }^{33}$ Neighbor corrected random coil chemical shift values from SPARTA+ have been subtracted from both simulated and experimental chemical shifts to generate secondary chemical shift data ${ }^{11}$. 


\section{Comparisons of SIC1 Data}
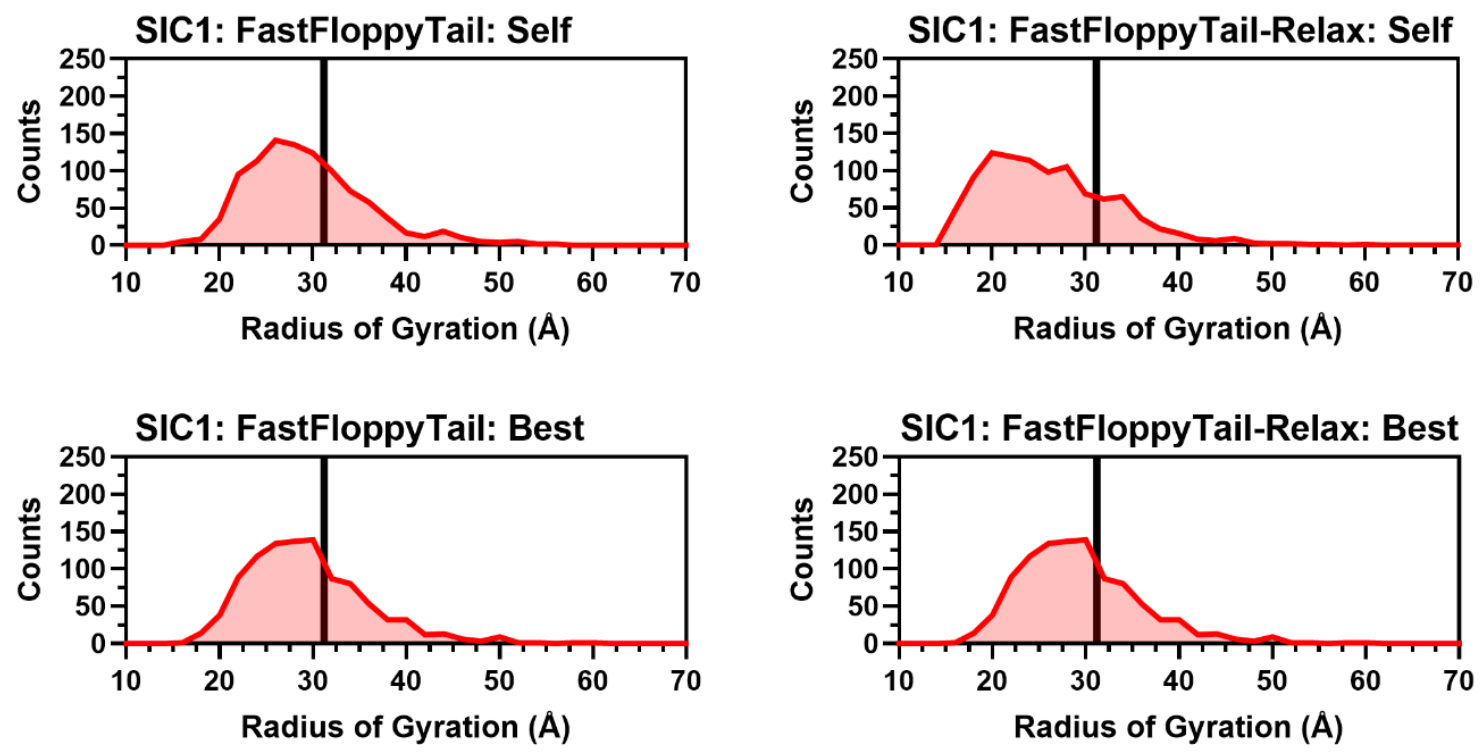

Figure S210: Histograms of Radii of Gyration for SIC1. Histograms of the radius of gyration from self (Top Left) and best (Top Right) reweighting in FastFloppyTail and self (Bottom Left) and best (Bottom Right) reweighting in FastFloppyTail-Relax compared to experimental values (black) reported by Mittag et al. ${ }^{34}$.
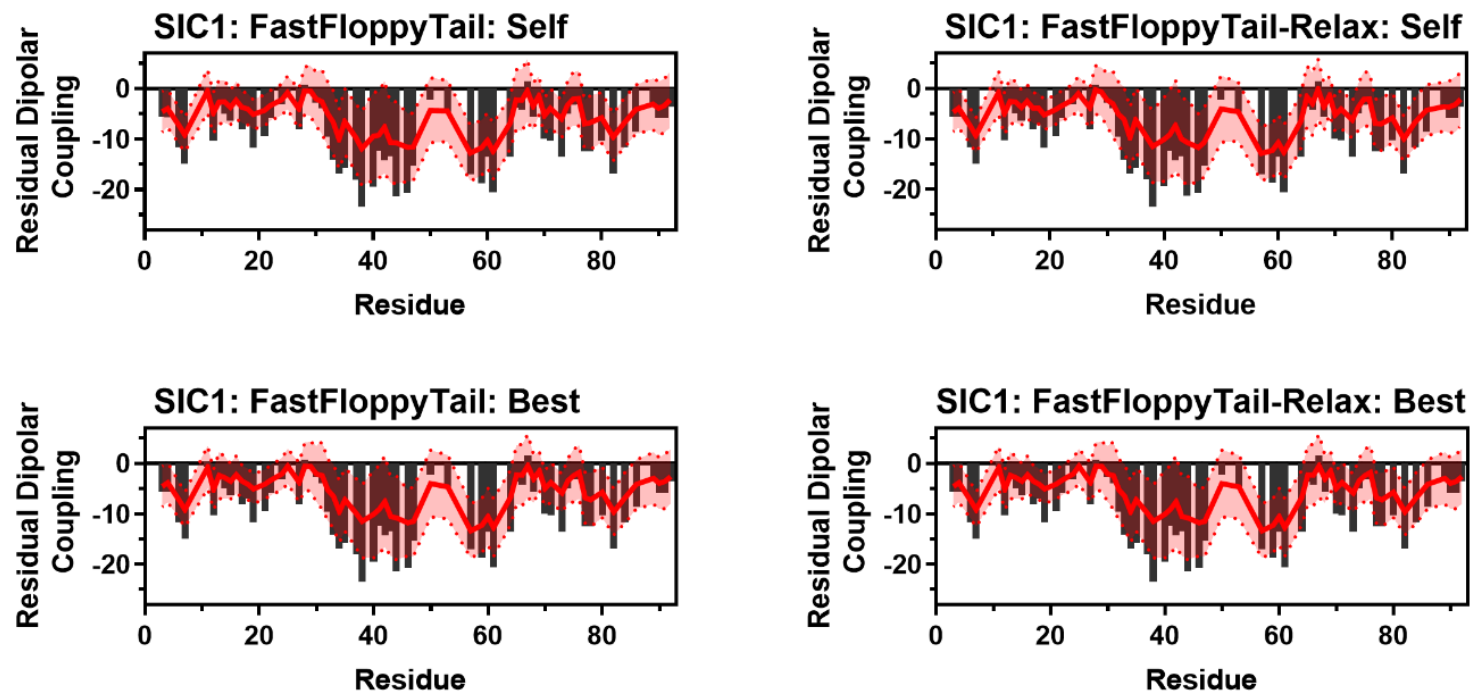

Figure S211: Comparison of Simulated and Experimental Residual Dipolar Coupling Data. Simulated RDC values (red line) from self (Top Left) and best (Top Right) reweighting in FastFloppyTail and self (Bottom Left) and best (Bottom Right) reweighting in FastFloppyTailRelax overlayed on experimental data (black bars) from Mittag et al. ${ }^{34}$. 


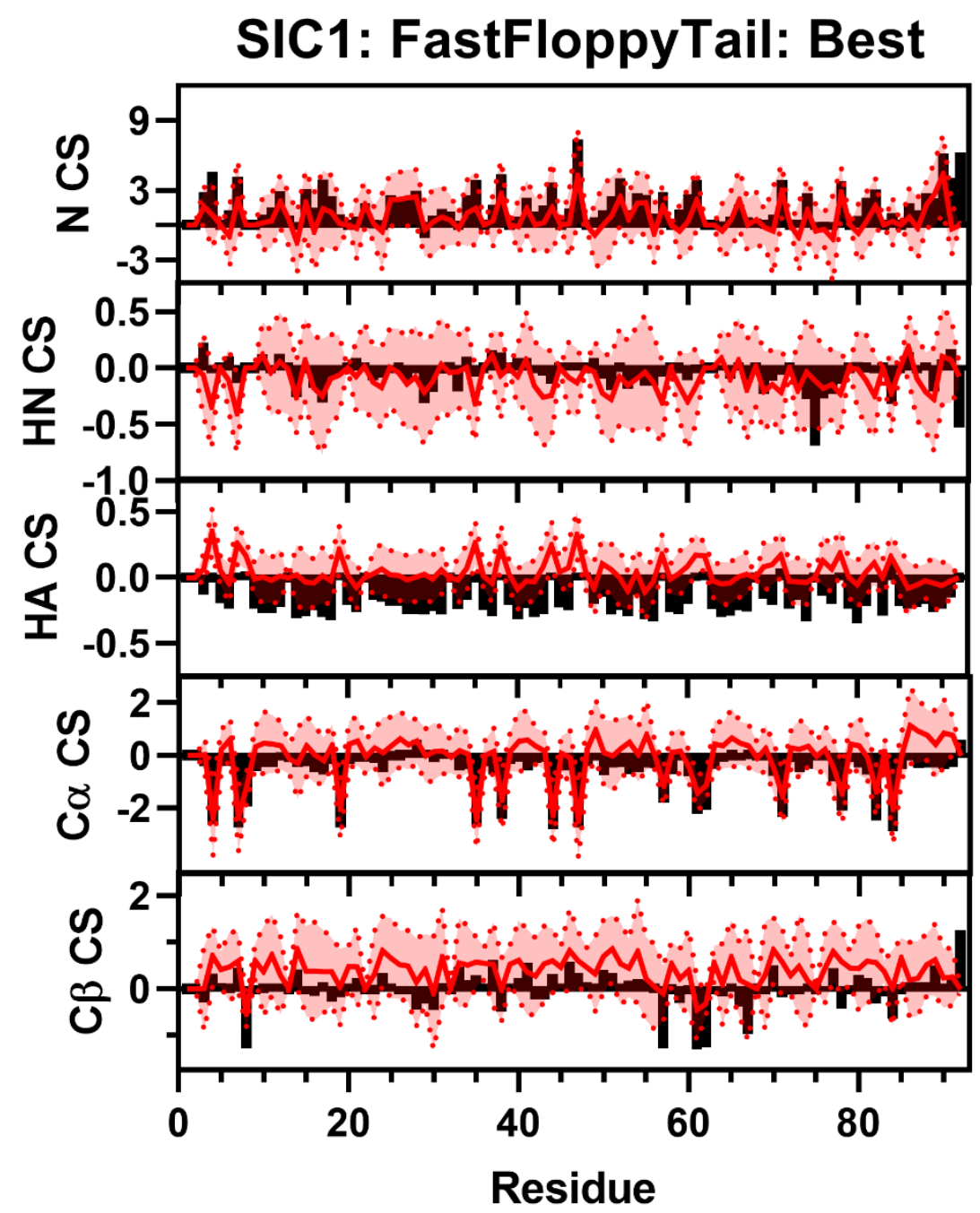

Figure S212: Comparison of Simulated and Experimental NMR Secondary Chemical Shift Data for SIC1. Simulated Chemical Shift values from the FastFloppyTail ensemble using bestreweighted fragment selection (red) overlayed on experimental data (black bars) of $\mathrm{N}$ (Row 1), HN (Row 2), HA (Row 3), C $\alpha$ (Row 4), and C $\beta$ (Row 5) chemical shifts from Mittag et al. ${ }^{34}$ Neighbor corrected random coil chemical shift values from Sparta + have been subtracted from both simulated and experimental chemical shifts to generate secondary chemical shift data ${ }^{11}$. 


\section{SIC1: FastFloppyTail-Relax: Best}

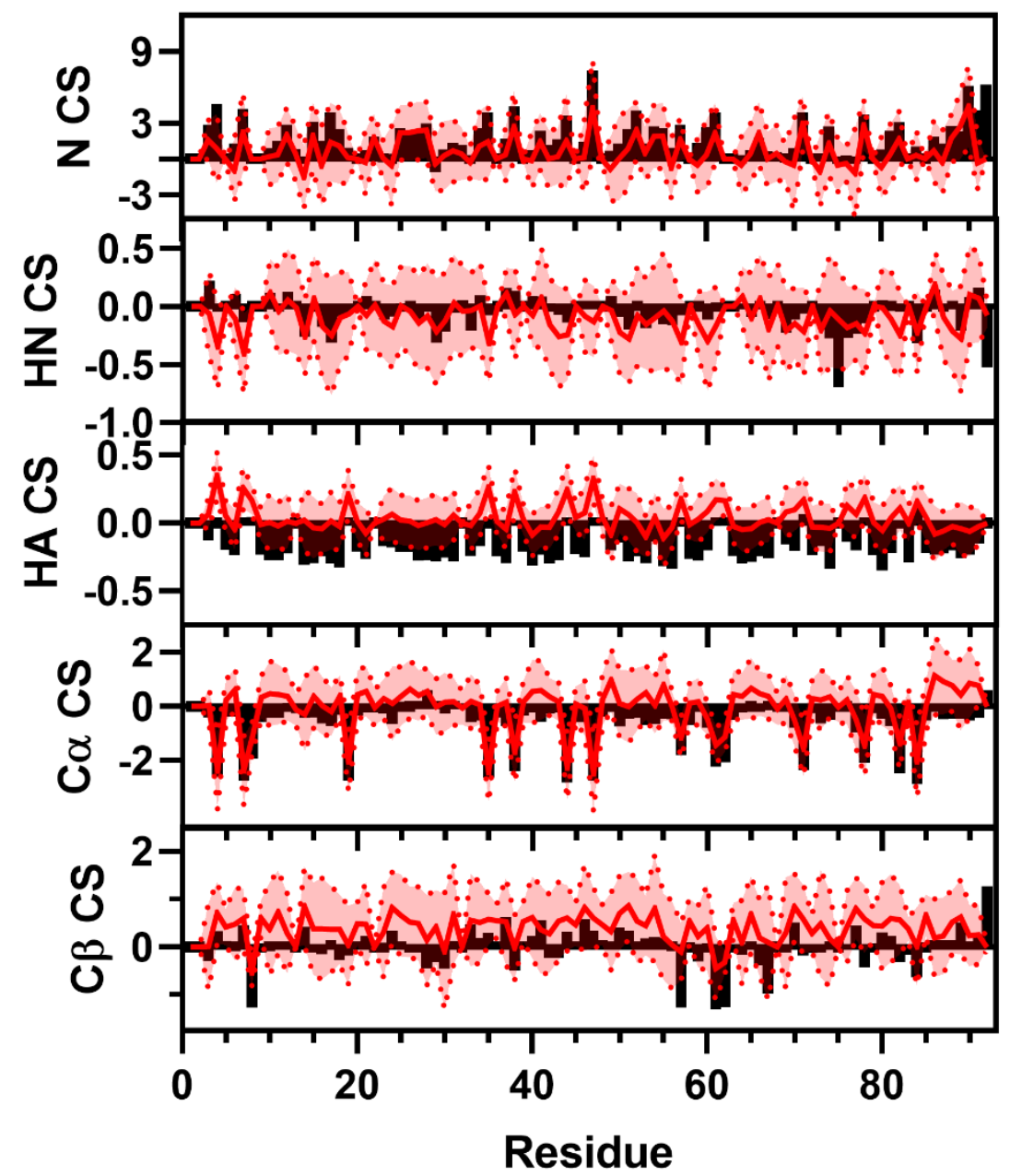

Figure S213: Comparison of Simulated and Experimental NMR Secondary Chemical Shift Data for SIC1. Simulated Chemical Shift values from the FastFloppyTail ensemble using bestreweighted fragment selection (red) overlayed on experimental data (black bars) of N (Row 1), HN (Row 2), HA (Row 3), C $\alpha$ (Row 4), and C $\beta$ (Row 5) chemical shifts from Mittag et al. ${ }^{34}$ Neighbor corrected random coil chemical shift values from Sparta + have been subtracted from both simulated and experimental chemical shifts to generate secondary chemical shift data ${ }^{11}$. 


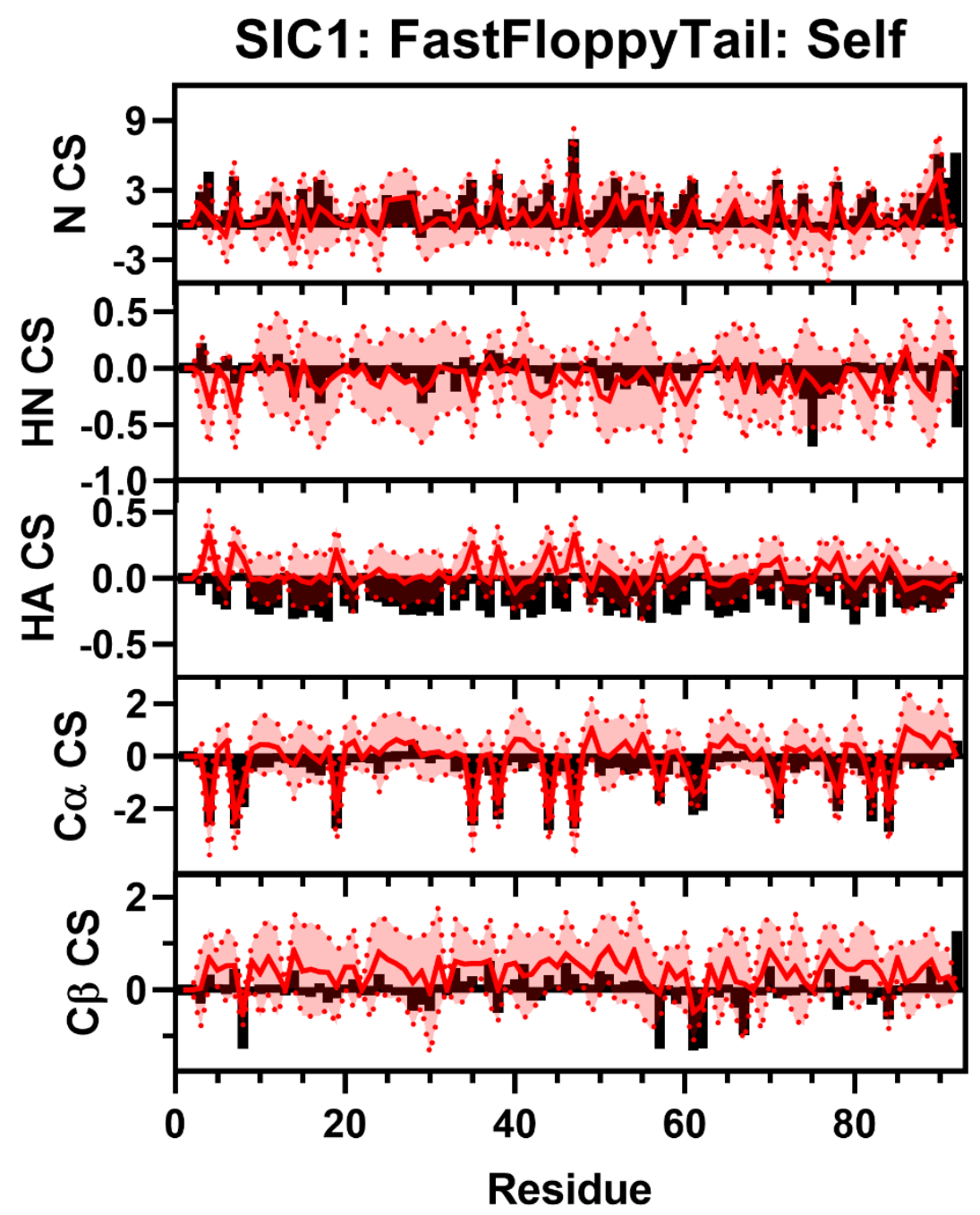

Figure S214: Comparison of Simulated and Experimental NMR Secondary Chemical Shift Data for SIC1. Simulated Chemical Shift values from the FastFloppyTail ensemble using bestreweighted fragment selection (red) overlayed on experimental data (black bars) of N (Row 1), HN (Row 2), HA (Row 3), C $\alpha$ (Row 4), and C $\beta$ (Row 5) chemical shifts from Mittag et al. ${ }^{34}$ Neighbor corrected random coil chemical shift values from Sparta + have been subtracted from both simulated and experimental chemical shifts to generate secondary chemical shift data ${ }^{11}$. 


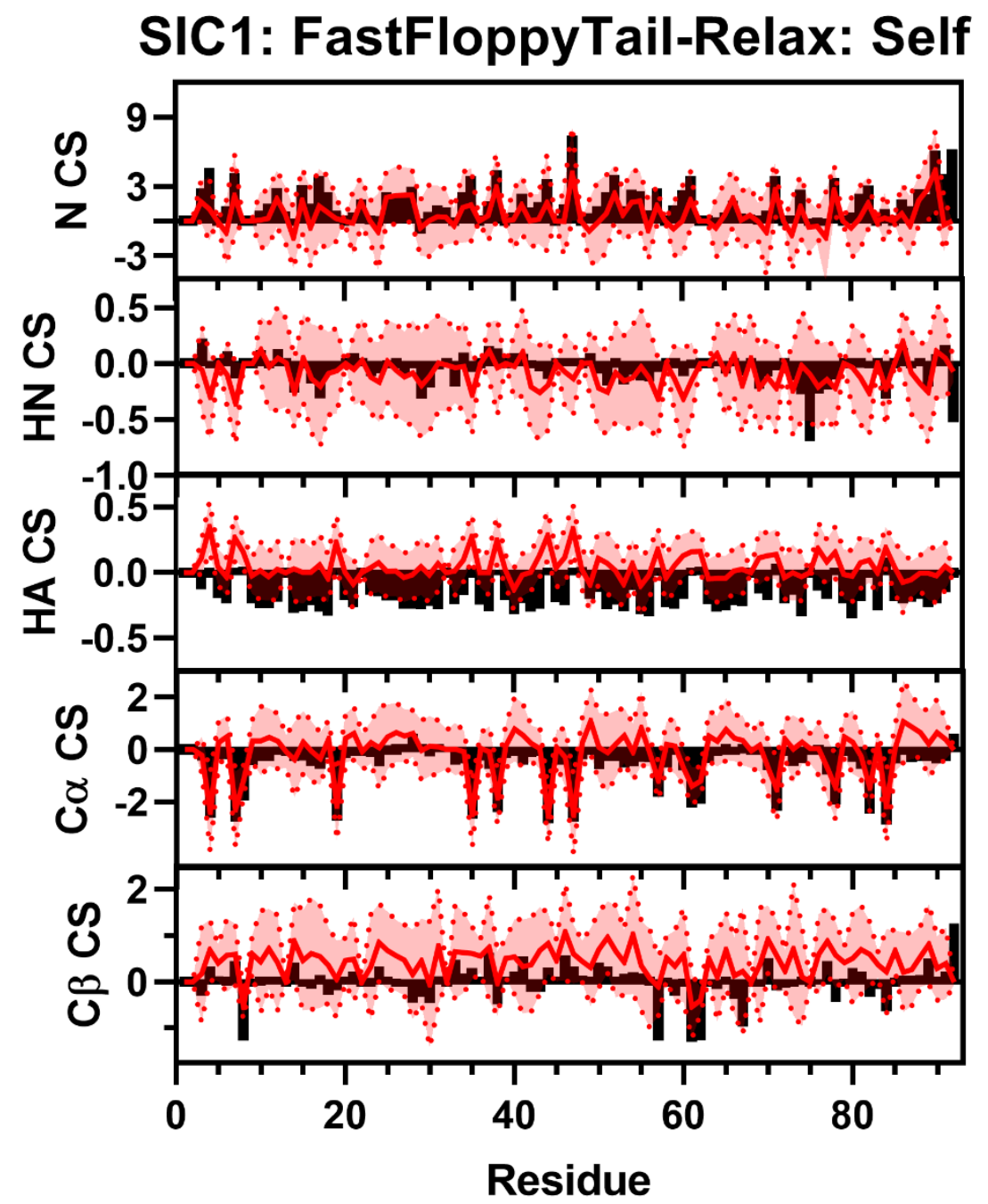

Figure S215: Comparison of Simulated and Experimental NMR Secondary Chemical Shift Data for SIC1. Simulated Chemical Shift values from the FastFloppyTail ensemble using bestreweighted fragment selection (red) overlayed on experimental data (black bars) of $\mathrm{N}$ (Row 1), HN (Row 2), HA (Row 3), C $\alpha$ (Row 4), and C $\beta$ (Row 5) chemical shifts from Mittag et al. ${ }^{34}$ Neighbor corrected random coil chemical shift values from Sparta + have been subtracted from both simulated and experimental chemical shifts to generate secondary chemical shift data ${ }^{11}$. 


\section{Comparisons of TAUK Data}
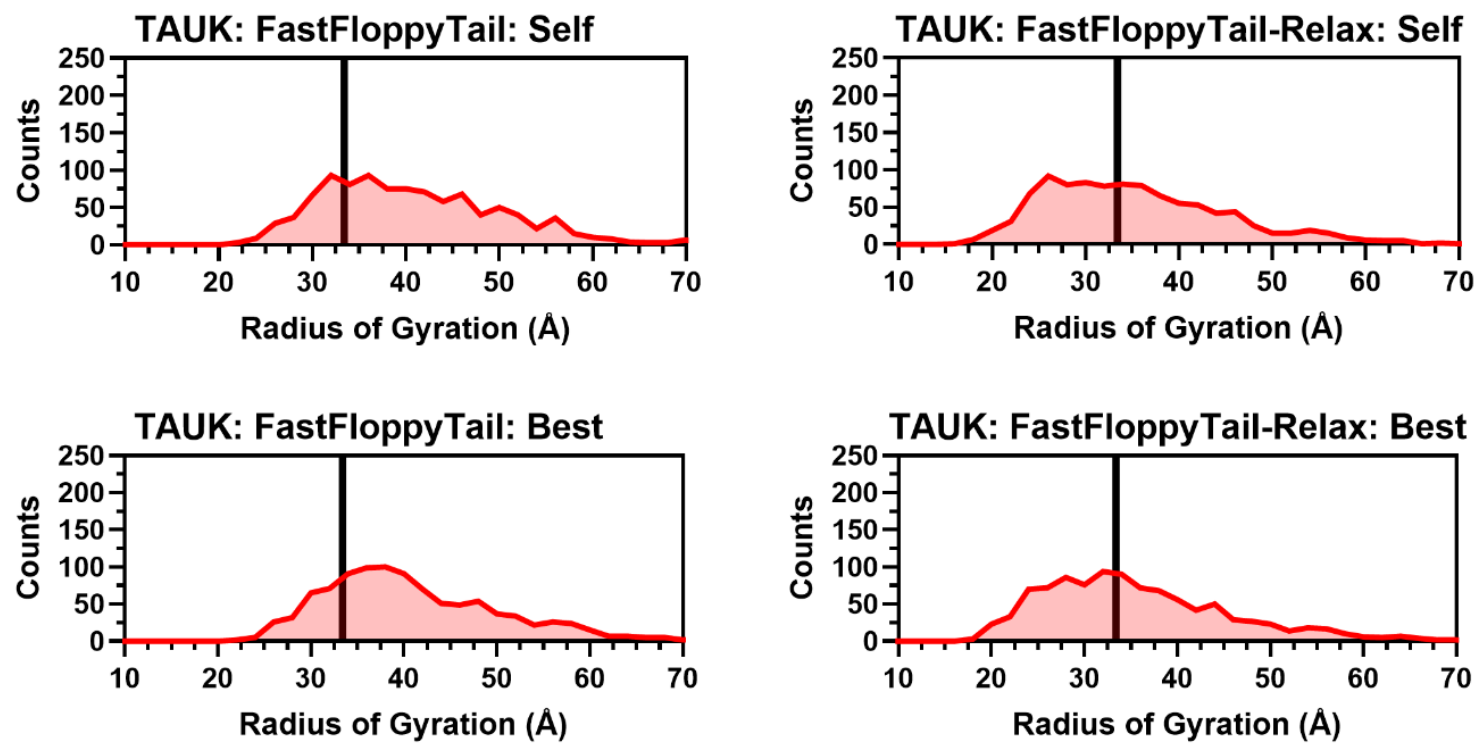

Figure S216: Histograms of Radii of Gyration for TAUK. Histograms of the radius of gyration from self (Top Left) and best (Top Right) reweighting in FastFloppyTail and self (Bottom Left) and best (Bottom Right) reweighting in FastFloppyTail-Relax compared to experimental values from Ozenne et al. (black) ${ }^{35}$.
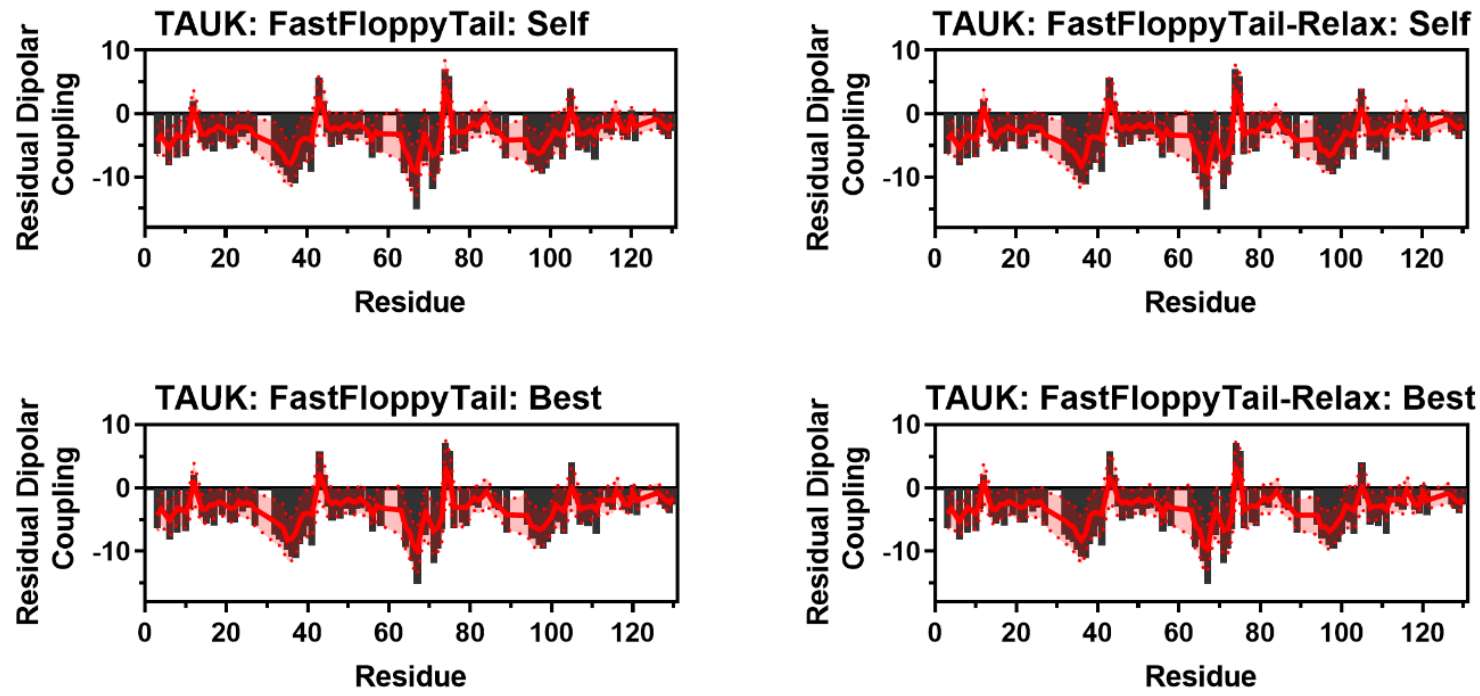

Figure S217: Comparison of Simulated and Experimental Residual Dipolar Coupling Data. Simulated RDC values (red line) from self (Top Left) and best (Top Right) reweighting in FastFloppyTail and self (Bottom Left) and best (Bottom Right) reweighting in FastFloppyTailRelax overlayed on experimental data (black bars) from Ozenne et al. ${ }^{35}$. 


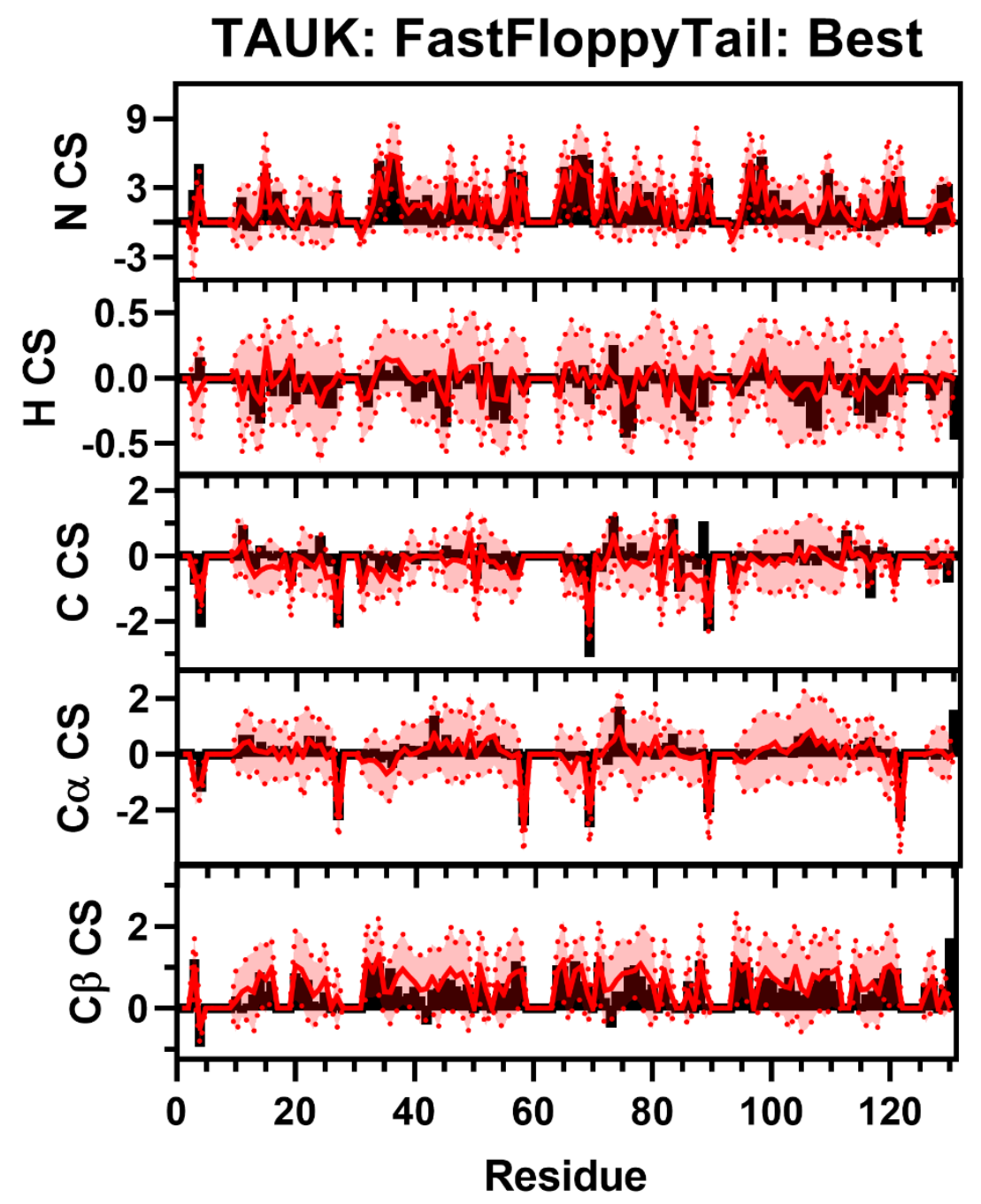

Figure S218: Comparison of Simulated and Experimental NMR Secondary Chemical Shift Data for TAUK. Simulated Chemical Shift values from the FastFloppyTail ensemble using bestreweighted fragment selection (red) overlayed on experimental data (black bars) of $\mathrm{N}$ (Row 1), $\mathrm{H}$ (Row 2), C (Row 3), C $\alpha$ (Row 4), and C $\beta$ (Row 5) chemical shifts from Ozenne et al. ${ }^{35}$. Neighbor corrected random coil chemical shift values from SPARTA+ have been subtracted from both simulated and experimental chemical shifts to generate secondary chemical shift data ${ }^{11}$. 


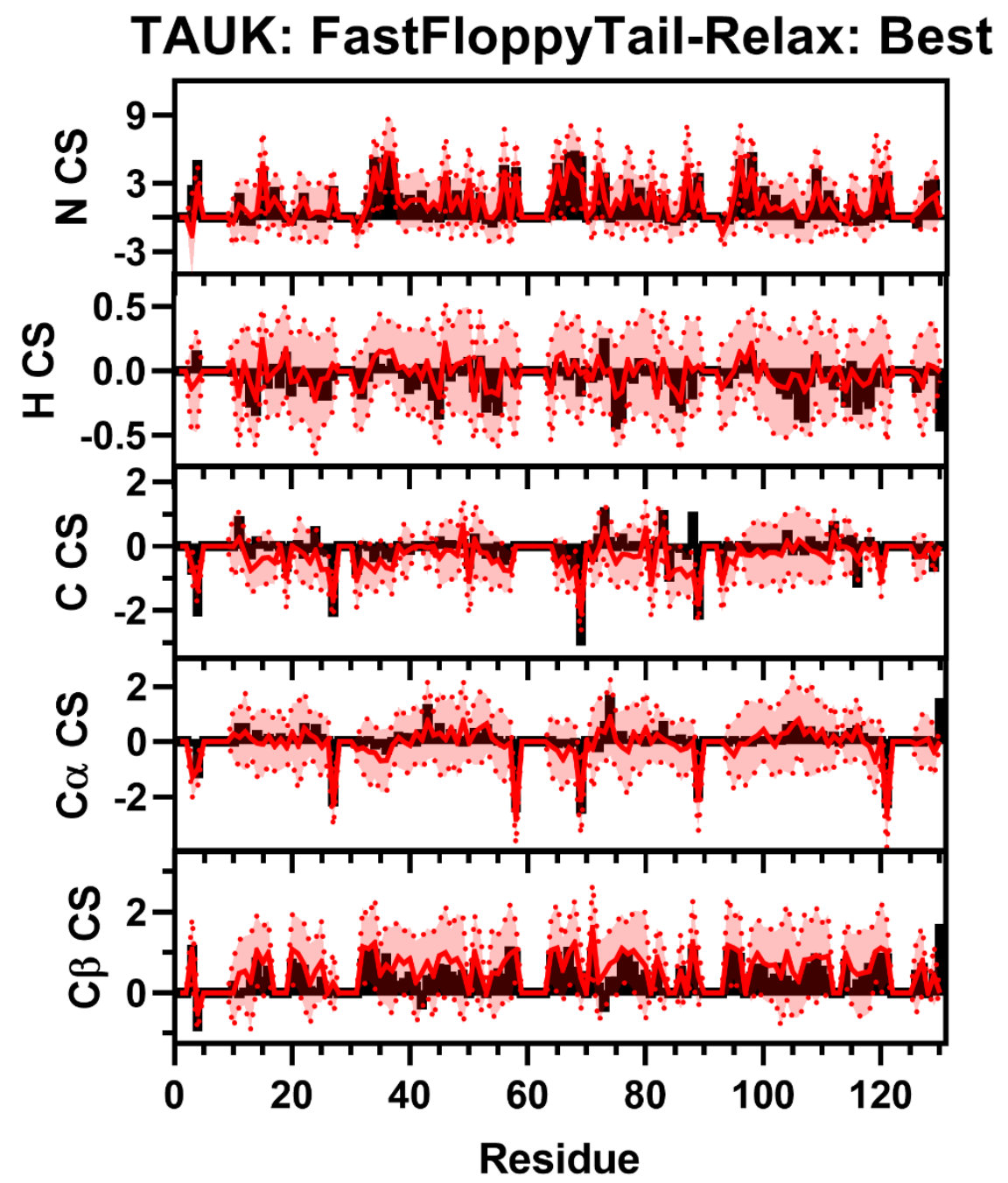

Figure S219: Comparison of Simulated and Experimental NMR Secondary Chemical Shift Data for TAUK. Simulated Chemical Shift values from the FastFloppyTail-Relax ensemble using bestreweighted fragment selection (red) overlayed on experimental data (black bars) of $\mathrm{N}$ (Row 1), $\mathrm{H}$ (Row 2), C (Row 3), C $\alpha$ (Row 4), and C $\beta$ (Row 5) chemical shifts from Ozenne et al. ${ }^{35}$ Neighbor corrected random coil chemical shift values from SPARTA+ have been subtracted from both simulated and experimental chemical shifts to generate secondary chemical shift data ${ }^{11}$. 


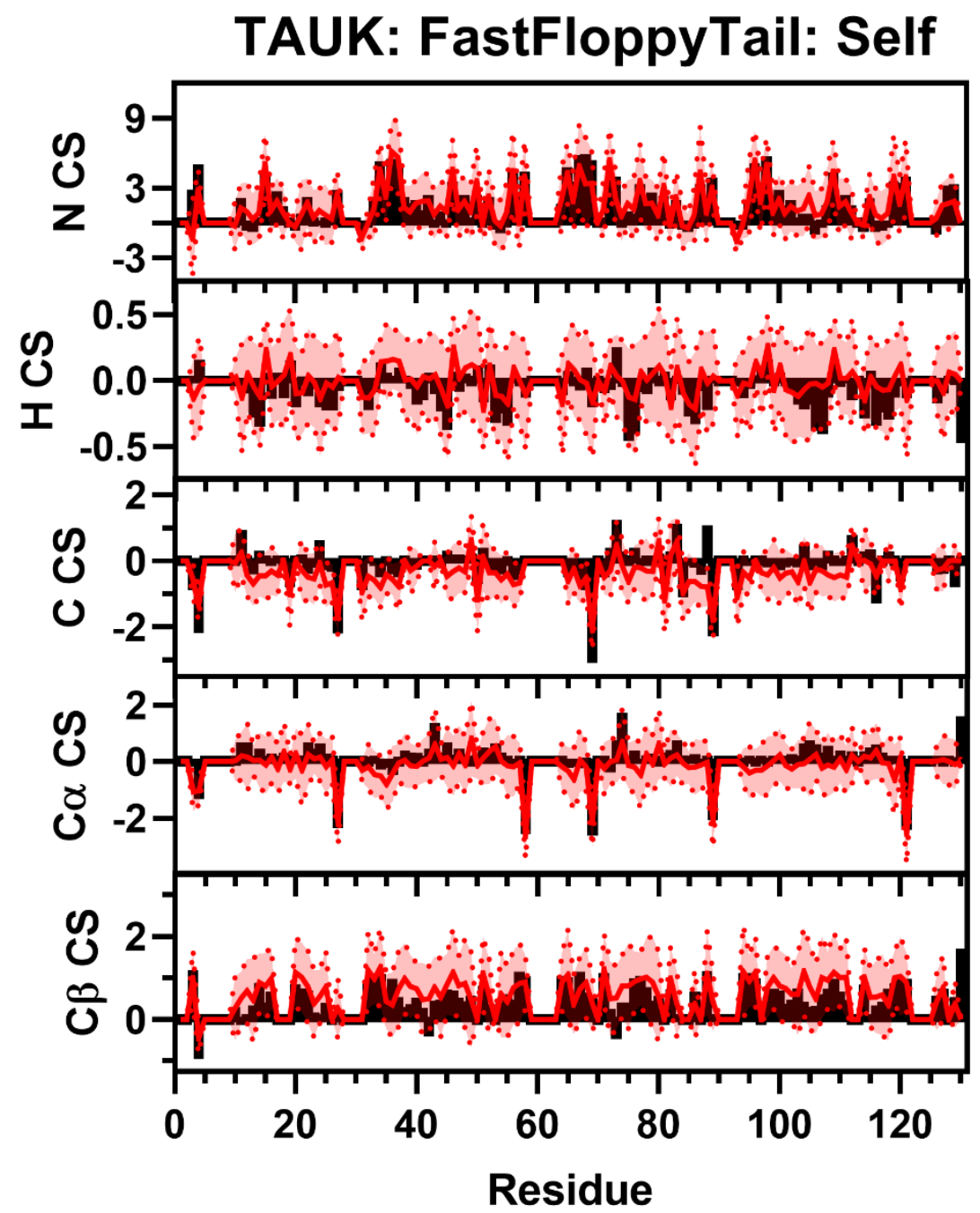

Figure S220: Comparison of Simulated and Experimental NMR Secondary Chemical Shift Data for TAUK. Simulated Chemical Shift values from the FastFloppyTail ensemble using selfreweighted fragment selection (red) overlayed on experimental data (black bars) of $\mathrm{N}$ (Row 1), $\mathrm{H}$ (Row 2), C (Row 3), C $\alpha$ (Row 4), and C $\beta$ (Row 5) chemical shifts from Ozenne et al. ${ }^{35}$ Neighbor corrected random coil chemical shift values from SPARTA+ have been subtracted from both simulated and experimental chemical shifts to generate secondary chemical shift data ${ }^{11}$. 


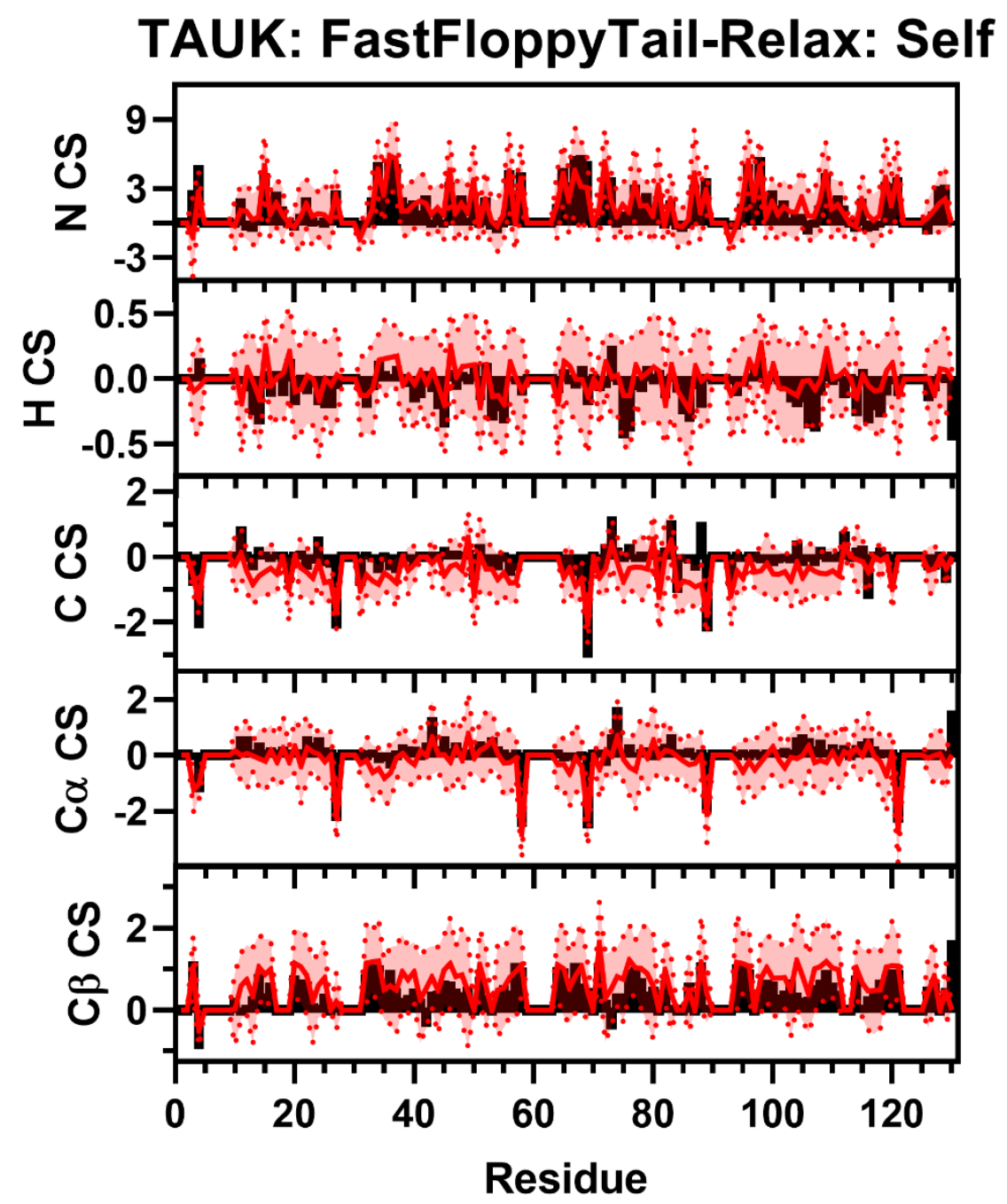

Figure S221: Comparison of Simulated and Experimental NMR Secondary Chemical Shift Data for TAUK. Simulated Chemical Shift values from the FastFloppyTail-Relax ensemble using selfreweighted fragment selection (red) overlayed on experimental data (black bars) of $\mathrm{N}$ (Row 1), $\mathrm{H}$ (Row 2), C (Row 3), C $\alpha$ (Row 4), and C $\beta$ (Row 5) chemical shifts from Ozenne et al. ${ }^{35}$ Neighbor corrected random coil chemical shift values from SPARTA+ have been subtracted from both simulated and experimental chemical shifts to generate secondary chemical shift data ${ }^{11}$. 


\section{References:}

1. Nath, A.; Sammalkorpi, M.; DeWitt, David C.; Trexler, Adam J.; Elbaum-Garfinkle, S.; O’Hern, Corey S.; Rhoades, E., The Conformational Ensembles of $\alpha$-Synuclein and Tau: Combining Single-Molecule FRET and Simulations. Biophysical Journal 2012, 103 (9), 19401949.

2. Kim, D. E.; Chivian, D.; Baker, D., Protein structure prediction and analysis using the Robetta server. Nucleic Acids Research 2004, 32 (Web Server issue), W526-W531.

3. Gront, D.; Kulp, D. W.; Vernon, R. M.; Strauss, C. E. M.; Baker, D., Generalized Fragment Picking in Rosetta: Design, Protocols and Applications. PLoS One 2011, 6 (8), e23294.

4. $\quad$ Leman, J. K.; Ralf, M.; Mert, K.; Nils, W.; Jens, M., Simultaneous prediction of protein secondary structure and transmembrane spans. Proteins 2013, 81 (7), 1127-1140.

5. Buchan, D. W. A.; Minneci, F.; Nugent, T. C. O.; Bryson, K.; Jones, D. T., Scalable web services for the PSIPRED Protein Analysis Workbench. Nucleic Acids Research 2013, 41 (Web Server issue), W349-W357.

6. Wang, S.; Li, W.; Liu, S.; Xu, J., RaptorX-Property: a web server for protein structure property prediction. Nucleic Acids Research 2016, 44 (W1), W430-W435.

7. Lakowicz, J. R., Principles of fluorescence spectroscopy. Second edition. New York : Kluwer Academic/Plenum, [1999] C)1999: 1999.

8. Sung, Y. H.; Eliezer, D., Residual structure, backbone dynamics, and interactions within the synuclein family. Journal of Molecular Biology 2007, 372 (3), 689-707.

9. Dedmon, M. M.; Lindorff-Larsen, K.; Christodoulou, J.; Vendruscolo, M.; Dobson, C. M., Mapping Long-Range Interactions in $\alpha$-Synuclein using Spin-Label NMR and Ensemble Molecular Dynamics Simulations. Journal of the American Chemical Society 2005, 127 (2), 476477.

10. Piana, S.; Donchev, A. G.; Robustelli, P.; Shaw, D. E., Water Dispersion Interactions Strongly Influence Simulated Structural Properties of Disordered Protein States. The Journal of Physical Chemistry B 2015, 119 (16), 5113-5123.

11. Shen, Y.; Bax, A., SPARTA+: a modest improvement in empirical NMR chemical shift prediction by means of an artificial neural network. J Biomol NMR 2010, 48 (1), 13-22.

12. Zweckstetter, M., NMR: prediction of molecular alignment from structure using the PALES software. Nature Protocols 2008, 3, 679.

13. Mantsyzov, A. B.; Maltsev, A. S.; Ying, J.; Shen, Y.; Hummer, G.; Bax, A., A maximum entropy approach to the study of residue-specific backbone angle distributions in $\alpha$-synuclein, an intrinsically disordered protein. Protein Science 2014, 23 (9), 1275-1290.

14. Kabsch, W.; Sander, C., Dictionary of protein secondary structure: pattern recognition of hydrogen-bonded and geometrical features. Biopolymers 1983, 22 (12), 2577-637.

15. Vögeli, B.; Ying, J.; Grishaev, A.; Bax, A., Limits on Variations in Protein Backbone Dynamics from Precise Measurements of Scalar Couplings. J. Am. Chem. Soc. 2007, 129 (30), 9377-9385.

16. Vuister, G. W.; Delaglio, F.; Bax, A., The use of $1 \mathrm{JC}$ alpha $\mathrm{H}$ alpha coupling constants as a probe for protein backbone conformation. J Biomol NMR 1993, 3 (1), 67-80.

17. Wirmer, J.; Schwalbe, H., Angular dependence of $1 \mathrm{~J}(\mathrm{Ni}, \mathrm{C} \alpha \mathrm{i})$ and $2 \mathrm{~J}(\mathrm{Ni}, \mathrm{C} \alpha(\mathrm{i}-1))$ coupling constants measured in J-modulated HSQCs. J. Biomol. NMR 2002, 23 (1), 47-55. 
18. Lee, J. H.; Li, F.; Grishaev, A.; Bax, A., Quantitative Residue-Specific Protein Backbone Torsion Angle Dynamics from Concerted Measurement of 3J Couplings. J. Am. Chem. Soc. 2015, 137 (4), 1432-1435.

19. Araki, K.; Yagi, N.; Nakatani, R.; Sekiguchi, H.; So, M.; Yagi, H.; Ohta, N.; Nagai, Y.; Goto, Y.; Mochizuki, H., A small-angle X-ray scattering study of alpha-synuclein from human red blood cells. Sci. Rep. 2016, 6, 30473.

20. Morar, A. S.; Olteanu, A.; Young, G. B.; Pielak, G. J., Solvent-induced collapse of $\alpha-$ synuclein and acid-denatured cytochrome c. Protein Science 2001, 10 (11), 2195-2199.

21. Ferrie, J. J.; Haney, C. M.; Yoon, J.; Pan, B.; Lin, Y. C.; Fakhraai, Z.; Rhoades, E.; Nath, A.; Petersson, E. J., Using a FRET Library with Multiple Probe Pairs To Drive Monte Carlo Simulations of alpha-Synuclein. Biophys J 2018, 114 (1), 53-64.

22. Ferrie, J. J.; Ieda, N.; Haney, C. M.; Walters, C. R.; Sungwienwong, I.; Yoon, J.; Petersson, E. J., Multicolor protein FRET with tryptophan, selective coumarin-cysteine labeling, and genetic acridonylalanine encoding. Chemical Communications 2017, 53 (80), 11072-11075.

23. Grupi, A.; Haas, E., Segmental conformational disorder and dynamics in the intrinsically disordered protein alpha-synuclein and its chain length dependence. J Mol Biol 2011, 405 (5), 1267-83.

24. Lee, J. C.; Gray, H. B.; Winkler, J. R., Tertiary Contact Formation in $\alpha$-Synuclein Probed by Electron Transfer. Journal of the American Chemical Society 2005, 127 (47), 16388-16389.

25. Bertoncini, C. W.; Jung, Y.-S.; Fernandez, C. O.; Hoyer, W.; Griesinger, C.; Jovin, T. M.; Zweckstetter, M., Release of long-range tertiary interactions potentiates aggregation of natively unstructured $\alpha$-synuclein. Proceedings of the National Academy of Sciences of the United States of America 2005, 102 (5), 1430-1435.

26. Camilloni, C.; De Simone, A.; Vranken, W. F.; Vendruscolo, M., Determination of secondary structure populations in disordered states of proteins using nuclear magnetic resonance chemical shifts. Biochemistry 2012, 51 (11), 2224-31.

27. Zheng, W.; Zerze, G. H.; Borgia, A.; Mittal, J.; Schuler, B.; Best, R. B., Inferring properties of disordered chains from FRET transfer efficiencies. The Journal of Chemical Physics 2018, 148 (12), 123329.

28. Hofmann, H.; Soranno, A.; Borgia, A.; Gast, K.; Nettels, D.; Schuler, B., Polymer scaling laws of unfolded and intrinsically disordered proteins quantified with single-molecule spectroscopy. Proceedings of the National Academy of Sciences 2012, 109 (40), 16155-16160.

29. Millman, K. J.; Aivazis, M., Python for Scientists and Engineers. Comput. Sci. Eng. 2011, 13 (2), 9-12.

30. Bezsonova, I.; Singer, A.; Choy, W.-Y.; Tollinger, M.; Forman-Kay, J. D., Structural Comparison of the Unstable drkN SH3 Domain and a Stable Mutant. Biochemistry 2005, 44 (47), 15550-15560.

31. Marsh, J. A.; Forman-Kay, J. D., Structure and Disorder in an Unfolded State under Nondenaturing Conditions from Ensemble Models Consistent with a Large Number of Experimental Restraints. J. Mol. Biol. 2009, 391 (2), 359-374.

32. Jensen, M. R.; Communie, G.; Ribeiro, E. A.; Martinez, N.; Desfosses, A.; Salmon, L.; Mollica, L.; Gabel, F.; Jamin, M.; Longhi, S., et al., Intrinsic disorder in measles virus nucleocapsids. Proc. Natl.Acad. Sci. USA 2011, 108 (24), 9839-9844.

33. Sterckx, Yann G. J.; Volkov, Alexander N.; Vranken, Wim F.; Kragelj, J.; Jensen, Malene R.; Buts, L.; Garcia-Pino, A.; Jové, T.; Van Melderen, L.; Blackledge, M., et al., Small- 
Angle X-Ray Scattering- and Nuclear Magnetic Resonance-Derived Conformational Ensemble of the Highly Flexible Antitoxin PaaA2. Structure 2014, 22 (6), 854-865.

34. Mittag, T.; Marsh, J.; Grishaev, A.; Orlicky, S.; Lin, H.; Sicheri, F.; Tyers, M.; FormanKay, J. D., Structure/Function Implications in a Dynamic Complex of the Intrinsically Disordered Sic1 with the Cdc4 Subunit of an SCF Ubiquitin Ligase. Structure 2010, 18 (4), 494506.

35. Ozenne, V.; Schneider, R.; Yao, M.; Huang, J.-r.; Salmon, L.; Zweckstetter, M.; Jensen, M. R.; Blackledge, M., Mapping the Potential Energy Landscape of Intrinsically Disordered Proteins at Amino Acid Resolution. J. Am. Chem. Soc. 2012, 134 (36), 15138-15148.

36. Robustelli, P.; Piana, S.; Shaw, D. E., Developing a molecular dynamics force field for both folded and disordered protein states. Proceedings of the National Academy of Sciences 2018.

37. Zhang, O.; Forman-Kay, J. D., Structural Characterization of Folded and Unfolded States of an SH3 Domain in Equilibrium in Aqueous Buffer. Biochemistry 1995, 34 (20), 6784-6794. 